\title{
Design and Synthesis of Novel c-di-GMP G-Quadruplex Inducers as Bacterial Biofilm Inhibitors
}

Teng-Fei Xuan ${ }^{\S}$, Zi-Qiang Wang ${ }^{\S}$, Jun Liu, Hai-Tao Yu, Qian-Wen Lin, Wei-Min Chen*, Jing Lin*

College of Pharmacy, Jinan University, Guangzhou 510632, P. R. China.

${ }^{\S}$ These authors contributed equally to this work.

*Corresponding authors. Tel.: +86 208522 1367(J. Lin), +86 2085224497 (W.-M. Chen).

Fax: +86 2085224766 .

E-mail address: linjing_jnu@163.com (J. Lin), twmchen@jnu.edu.cn(W.-M. Chen).

Table of contents

Determination of c-di-GMP Content in Different Strains. S2

Inhibitory effect of inducer 5h on PAO1 biofilm S3

Inhibitory effect of inducer 5h on ATCC 57853 biofilm S4

Supporting Methods S5

Synthesis and Characterization of the intermediates .S6

${ }^{1} \mathrm{H}$ NMR, ${ }^{13} \mathrm{C}$ NMR, HRMS, and HPLC analysis of final compounds ..S7 
Figure S1. The concentration level of c-di-GMP signal molecules in different bacteria was determined by HPLC.

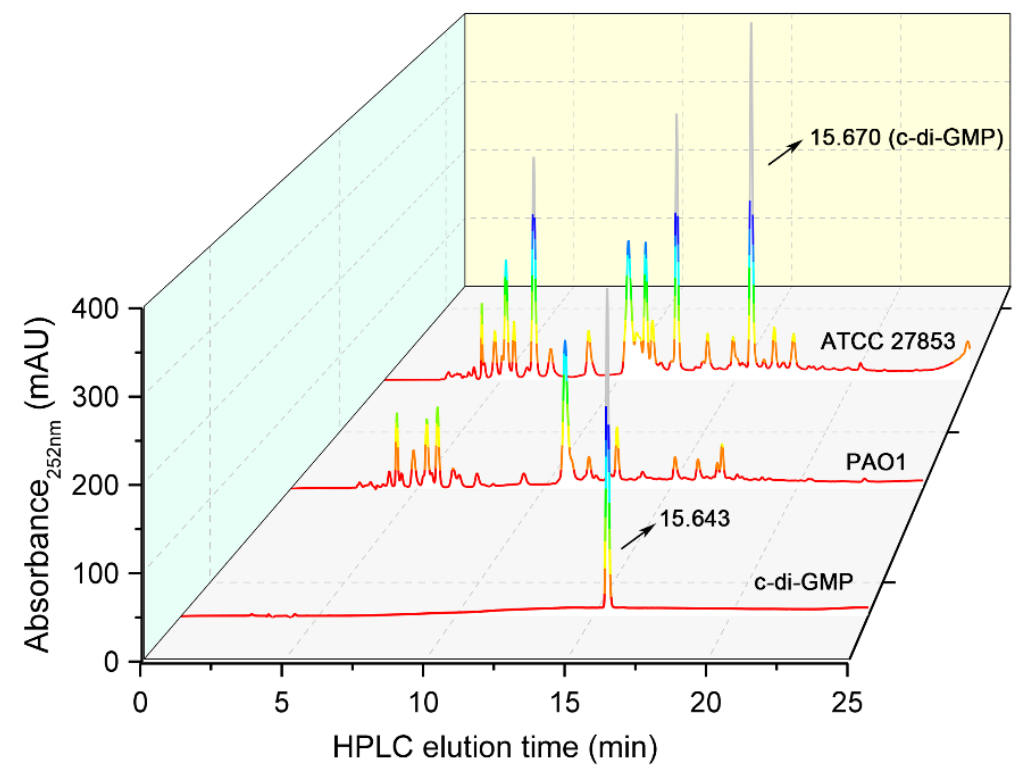


Figure S2. Inhibitory effect of inducer $\mathbf{5 h}$ with different concentrations on biofilm formation of PAO1 strain.

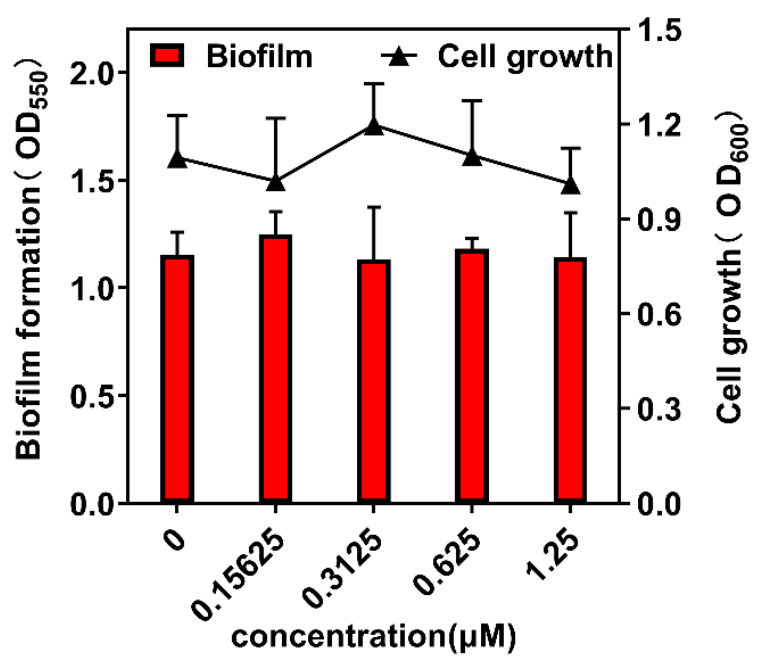


Figure S3. Inhibitory effect of inducer $\mathbf{5 h}$ with different concentrations on biofilm formation of ATCC 27853 strain.

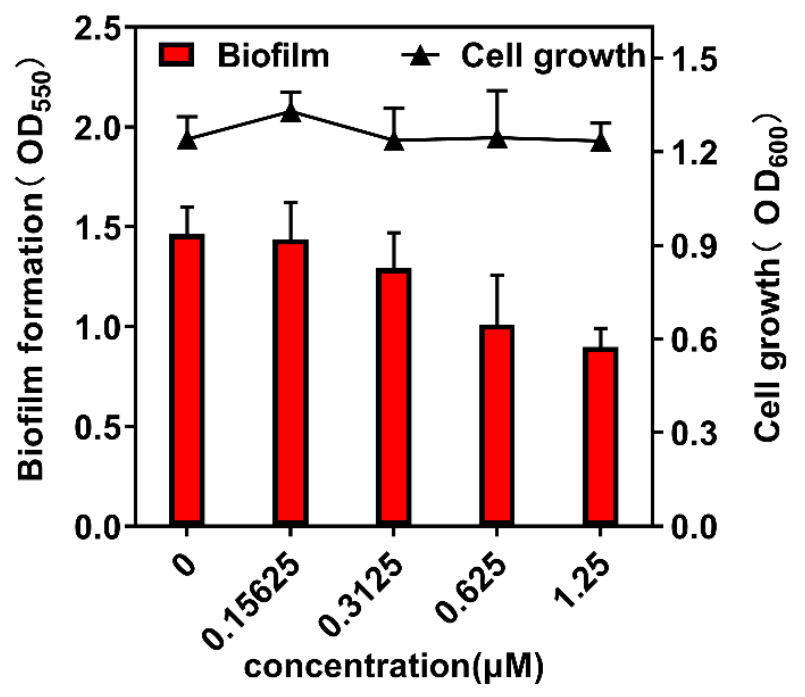




\section{Supporting Methods}

\section{Detection of amount of c-di-GMP in bacteria cell lysate using HPLC.}

Strains (PAO1 or ATCC27853) were grown up at $37{ }^{\circ} \mathrm{C}$ in Luria-Bertani (LB) medium $(500 \mathrm{~mL})$ with shaking for $16 \mathrm{~h}$. Cell were pelleted by centrifugation and resuspended to a final volume of $5 \mathrm{~mL}$ of $10 \mathrm{mM}$ Tris- $\mathrm{HCl}$ (pH 8.0) containing $100 \mathrm{mM} \mathrm{NaCl} .100$ $\mu \mathrm{L}$ of $25 \mathrm{mg} / \mathrm{mL}$ Lysozyme was added to each resuspension. The resuspension was sonicated $(10 \mathrm{~s} / 10 \mathrm{~s}, 30 \mathrm{~min})$ to disrupt membranes and allow lysozyme to reach cell walls. $60 \%$ perchloric acid (final concentration was 12\%) was added to the resuspension to precipitate cellular macromolecules. The resuspension was incubated for $10 \mathrm{~min}$ on ice and neutralized by $3 \mathrm{M} \mathrm{KOH}$ containing $0.4 \mathrm{M}$ Tris and $2 \mathrm{M} \mathrm{KCl}$. The resuspension was centrifuged, and the collected supernatant was then filtered by a $0.2 \mu \mathrm{m}$ filter and $3 \mathrm{kD}$ exclusion columns. $1 \mathrm{ml}$ of this lysate was used for the HPLC detection. HPLC conditions: A: $100 \mathrm{mM}$ of triethylammonium, B: $\mathrm{CH} 3 \mathrm{CN}, \mathrm{B} \%: 2 \%-2 \% / 3 \mathrm{~min}, 2 \%-$ $10 \% / 15 \mathrm{~min}, 10 \%-30 \% / 25 \mathrm{~min}, 30 \%-100 \% / 30 \mathrm{~min}$. Column: 5C18-AR (nacalai tech) at $1 \mathrm{~mL} / \mathrm{min}$ at room temperature. 
Table S1. Primer design information of RT-PCR experiment.

\begin{tabular}{ccc}
\hline Primer & Sequence $\left(\mathbf{5}^{\prime}\right.$-3' $)$ & Product $(\mathbf{b p})$ \\
\hline fleN-F1 & TCGGCAAGACCAATGTGTCG & \\
fleN-R1 & GCGTCCCTCGATCACGTC & 155 \\
flhA-F2 & TCGGCATGATCCAGCATTCG & \\
flhA-R2 & GAACATCTGCCGGTTGACCT & 182 \\
alg44-F1 & TTCGTCACCCATGCCGATTC & \\
alg44-R1 & GTCAGGTTGCCCTTGAGCAT & 170 \\
pelB-F2 & CGGAAATCCTCGAACGCACT & \\
pelB-R2 & CTACCGAGGAGCGAGCTATC & 187 \\
pslE-F1 & TGGAGAAATCGCGGATCGAC & \\
pslE-R1 & AGCAGGGTTTTCGGGAAGAT & 109 \\
cdrA-F2 & GGCTTCCTGGGACAACTACG & \\
cdrA-R2 & GATGAACGGATCGAACACCG & 121 \\
Rps1-F1 & TCGGCACTGCGTAAGGTATG & \\
Rps1-R1 & ACTACGCTGTGCTCTTGCAG & 101 \\
\hline & &
\end{tabular}




\section{Synthesis and Characterization of the intermediates 1, 4}

1,2-dimethylquinolin-1-ium iodide (1): Add methyl iodide $(0.42 \mathrm{~mL}, 6.74 \mathrm{mmol})$ to a solution of 2-methylquinoline $(0.5 \mathrm{~g}, 3.5 \mathrm{mmol})$ in sulfolane $(10 \mathrm{~mL})$. Then the mixture was reacted at $60^{\circ} \mathrm{C}$ for $18 \mathrm{~h}$, After cooling, the resulting precipitate was filtered, then washed with ethanol to give a yellow solid 0.9 g. yield 92\%. ${ }^{1} \mathrm{H}$ NMR (300 MHz, DMSO- $\left.d_{6}\right) \delta 9.39(\mathrm{~d}, J=6.0 \mathrm{~Hz}, 1 \mathrm{H}), 8.62-8.44(\mathrm{~m}, 2 \mathrm{H}), 8.28(\mathrm{~m}, 1 \mathrm{H}), 8.15-7.96(\mathrm{~m}$, 2H), $4.60(\mathrm{~s}, 3 \mathrm{H}), 3.01(\mathrm{~s}, 3 \mathrm{H}) .{ }^{13} \mathrm{C} \mathrm{NMR}\left(75 \mathrm{MHz}, \mathrm{DMSO}-d_{6}\right) \delta 158.63,149.12,138.14$, $135.41,130.12,128.94,127.27,122.94,120.02,45.58,20.14$.

2,3-dimethylbenzo[d]thiazol-3-ium iodide (4): 2-methyl benzothiazole $(0.76 \mathrm{~mL}, 6$ mmol) was dissolved in MeCN (10 mL). Methyl iodide (0.94 mL, $15 \mathrm{mmol})$ was added to the solution. Then the mixture was refluxed for $24 \mathrm{~h}$ under $\mathrm{N}_{2}$. After cooling, the resulting precipitate was filtered, then washed with $\mathrm{MeCN}$ and $\mathrm{Et}_{2} \mathrm{O}$ to give a white solid 1.42 g. yield 83\%. ${ }^{1} \mathrm{H}$ NMR (400 MHz, DMSO- $\left.d_{6}\right) \delta 8.49(\mathrm{~d}, J=8.1 \mathrm{~Hz}, 1 \mathrm{H})$, $8.28(\mathrm{~d}, J=8.4 \mathrm{~Hz}, 1 \mathrm{H}), 7.85(\mathrm{t}, J=7.8 \mathrm{~Hz}, 1 \mathrm{H}), 7.77(\mathrm{t}, J=7.7 \mathrm{~Hz}, 1 \mathrm{H}), 4.23(\mathrm{~s}, 3 \mathrm{H})$, $3.23(\mathrm{~s}, 3 \mathrm{H}) ;{ }^{13} \mathrm{C}$ NMR (100 MHz, DMSO- $\left.d_{6}\right) \delta 177.45,141.98,129.70,129.07,128.45$, 125.02, 117.26, 37.29, 18.40 . 
- ${ }^{1} \mathrm{H}$ NMR, ${ }^{13} \mathrm{C}$ NMR, HRMS, and HPLC analysis of final compounds

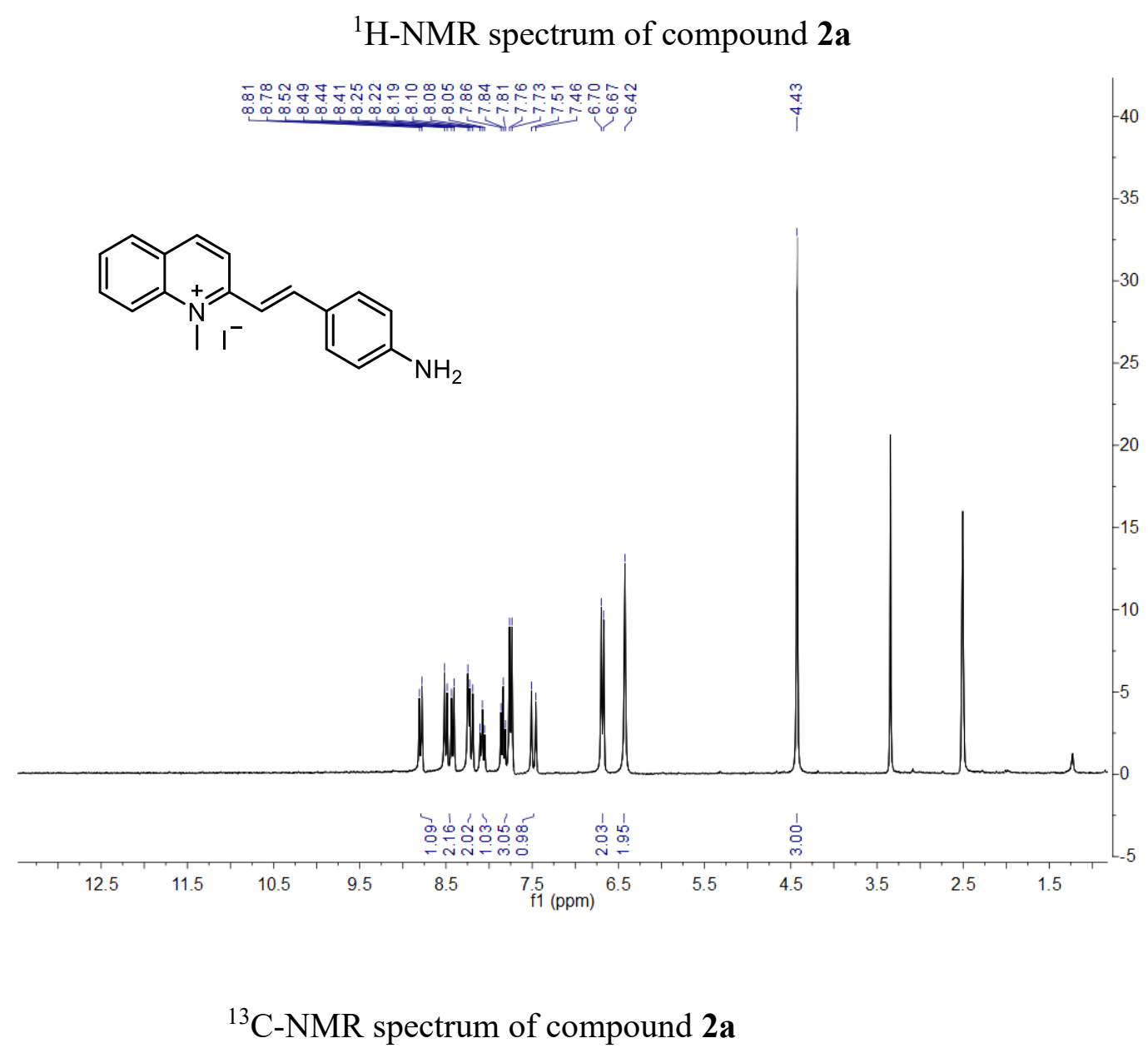




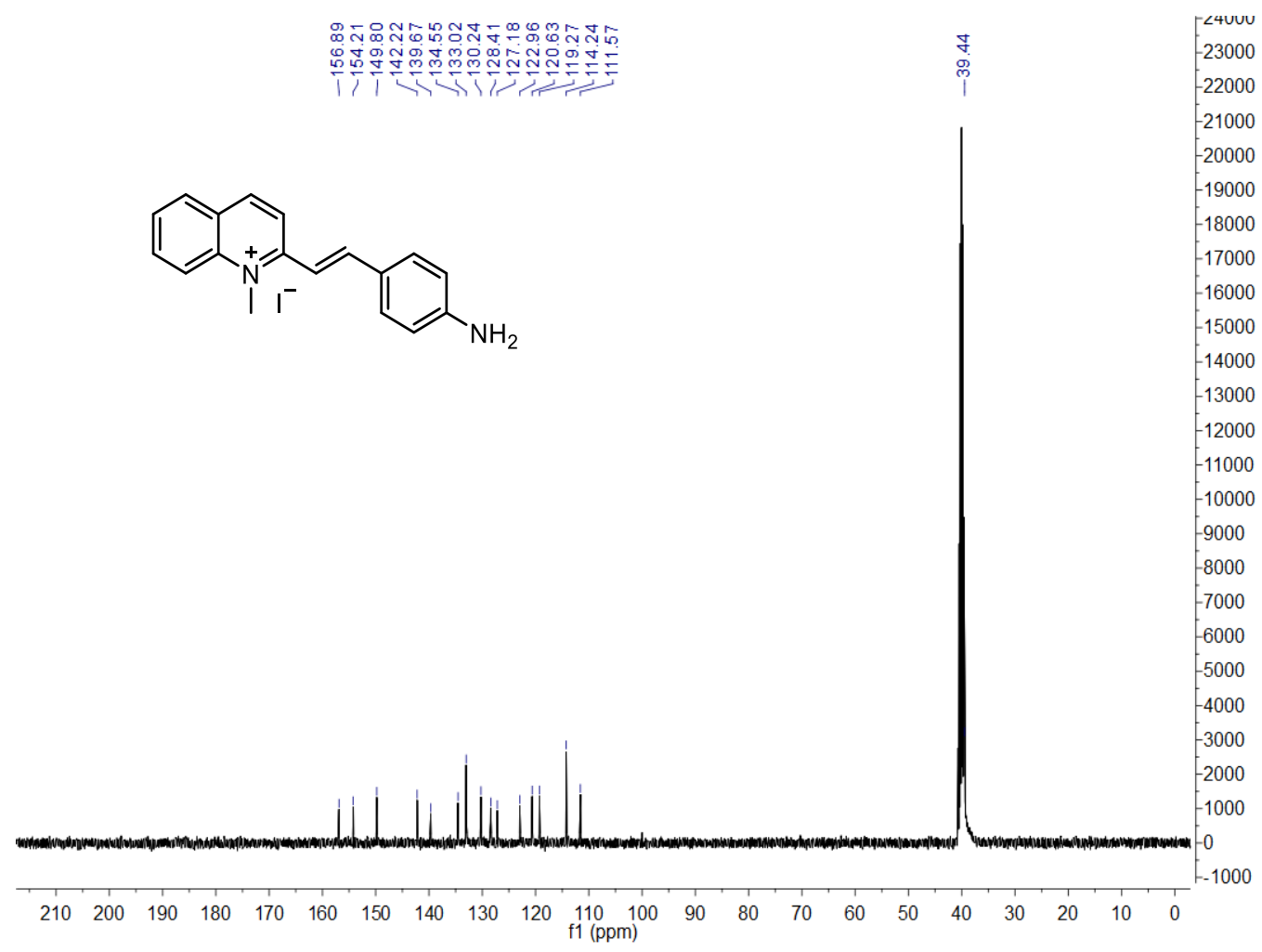

HRMS of compound 2a

Spectrum from 20200602.wiff (sample 68) - 133, Experiment 1, +TOF MS (50 - 1000) from 0.131 to $0.179 \mathrm{~min}$

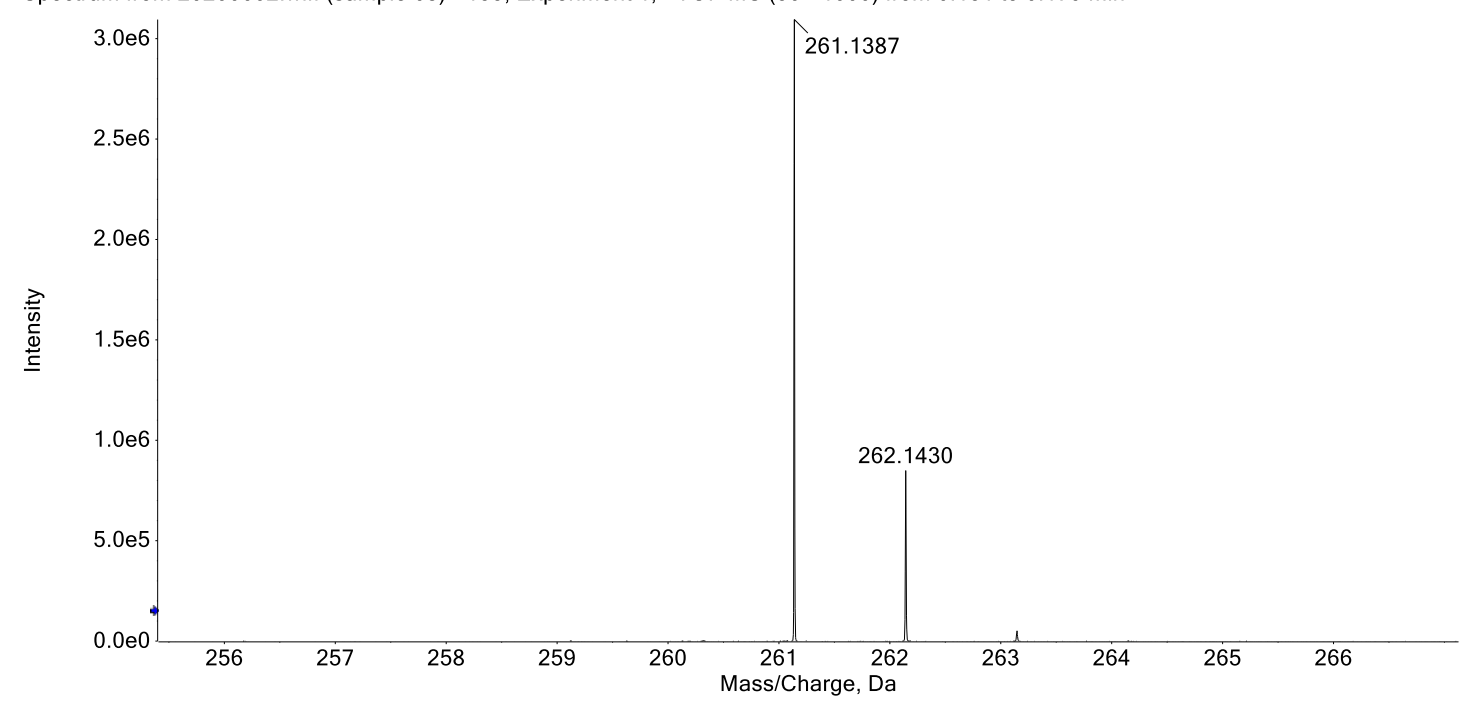

\begin{tabular}{|c|c|c|c|c|c|c|c|c|}
\hline Hit & Formula & $\mathrm{m} / \mathrm{z}$ & RDB & $\mathrm{ppm}$ & MS Rank & MSMS ppm & MSMS Rank & Found \\
\hline 1 & $\mathrm{C}_{18} \mathrm{H}_{17} \mathrm{~N}_{2}{ }^{+}$ & 261.1386 & 12.0 & 0.3 & 1 & & & NA/NA \\
\hline
\end{tabular}


HPLC analysis of $\mathbf{2 a}$

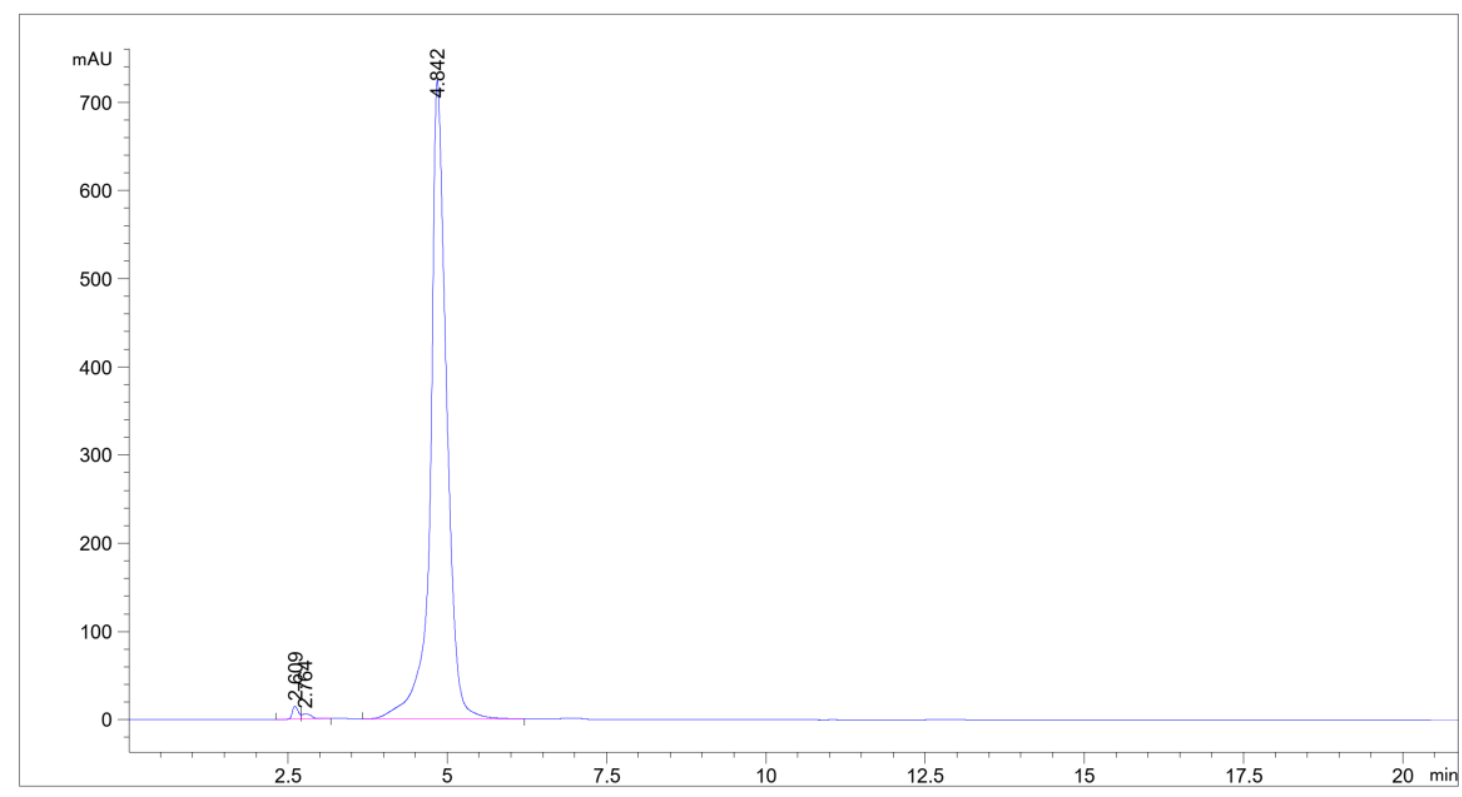

Area Percent Report

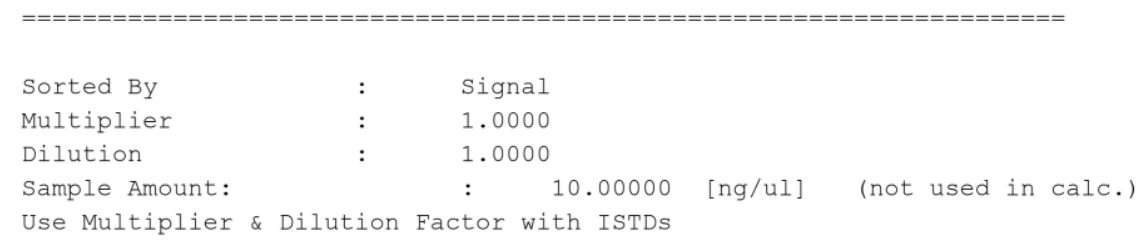

Signal 1: VWD1 A, Wavelength=254 nm

\begin{tabular}{|c|c|c|c|c|c|c|}
\hline $\begin{array}{c}\text { Peak } \\
\#\end{array}$ & $\begin{array}{c}\text { RetTime } \\
\text { [min] }\end{array}$ & Type & $\begin{array}{l}\text { Width } \\
\text { [min] }\end{array}$ & $\begin{array}{c}\text { Area } \\
{\left[\mathrm{mAU}^{\star} \mathrm{s}\right]}\end{array}$ & $\begin{array}{l}\text { Height } \\
\text { [mAU] }\end{array}$ & $\begin{array}{c}\text { Area } \\
\frac{8}{8}\end{array}$ \\
\hline$---\mid$ & |------- | & $1--$ & ------- & |-------- & $----------\mid$ & $-------\mid$ \\
\hline 1 & 2.609 & BV & 0.1049 & 99.69811 & 14.74802 & 0.7707 \\
\hline 2 & 2.764 & VB & 0.1355 & 59.78981 & 5.83587 & 0.4622 \\
\hline 3 & 4.842 & $\mathrm{BB}$ & 0.2549 & $1.27759 \mathrm{e} 4$ & 724.02234 & 98.7670 \\
\hline
\end{tabular}

Totals: $\quad 1.29354 \mathrm{e} 4 \quad 744.60622$

${ }^{1} \mathrm{H}-\mathrm{NMR}$ spectrum of compound $\mathbf{2 b}$ 


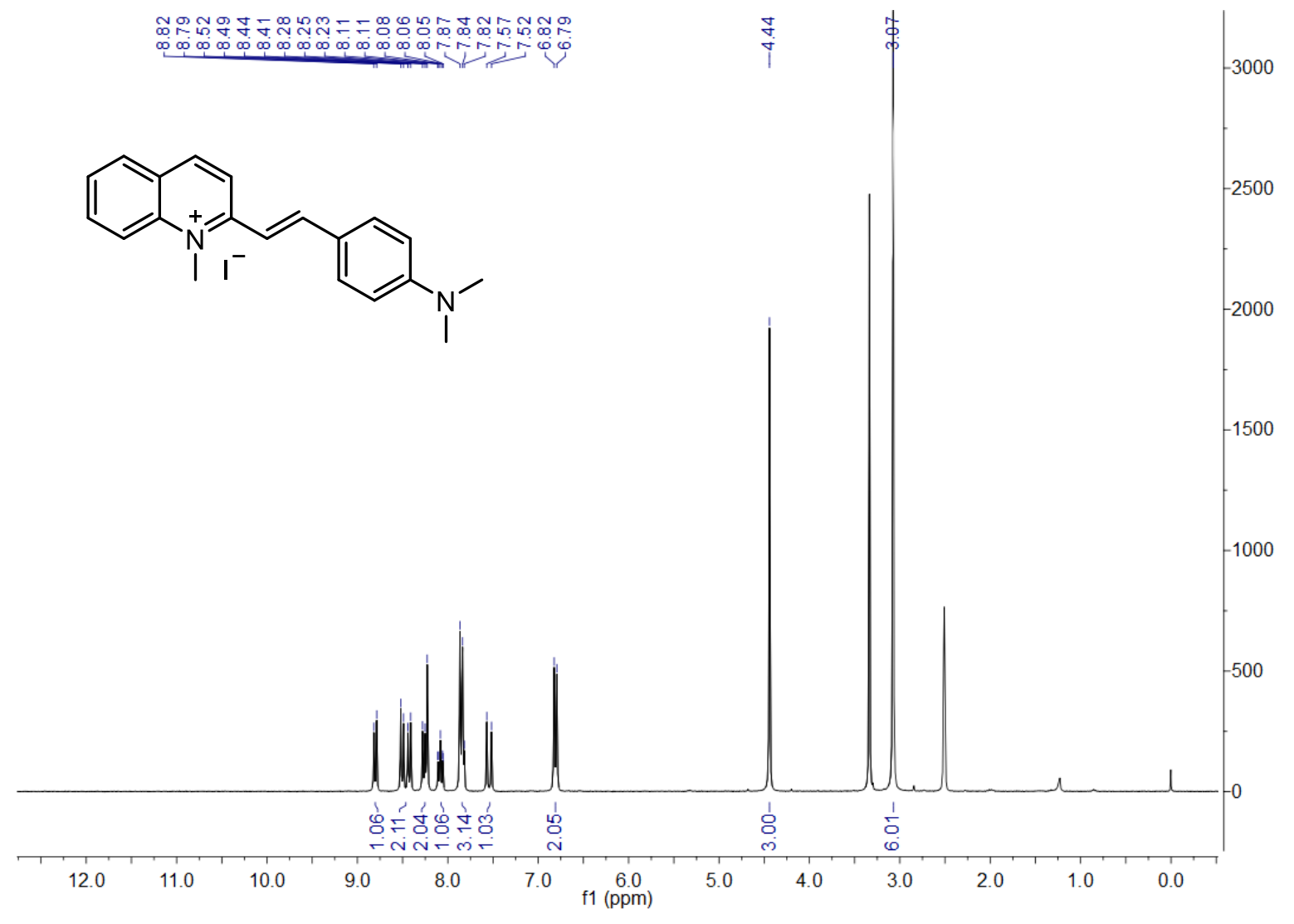

${ }^{13} \mathrm{C}$-NMR spectrum of compound $\mathbf{2 b}$ 


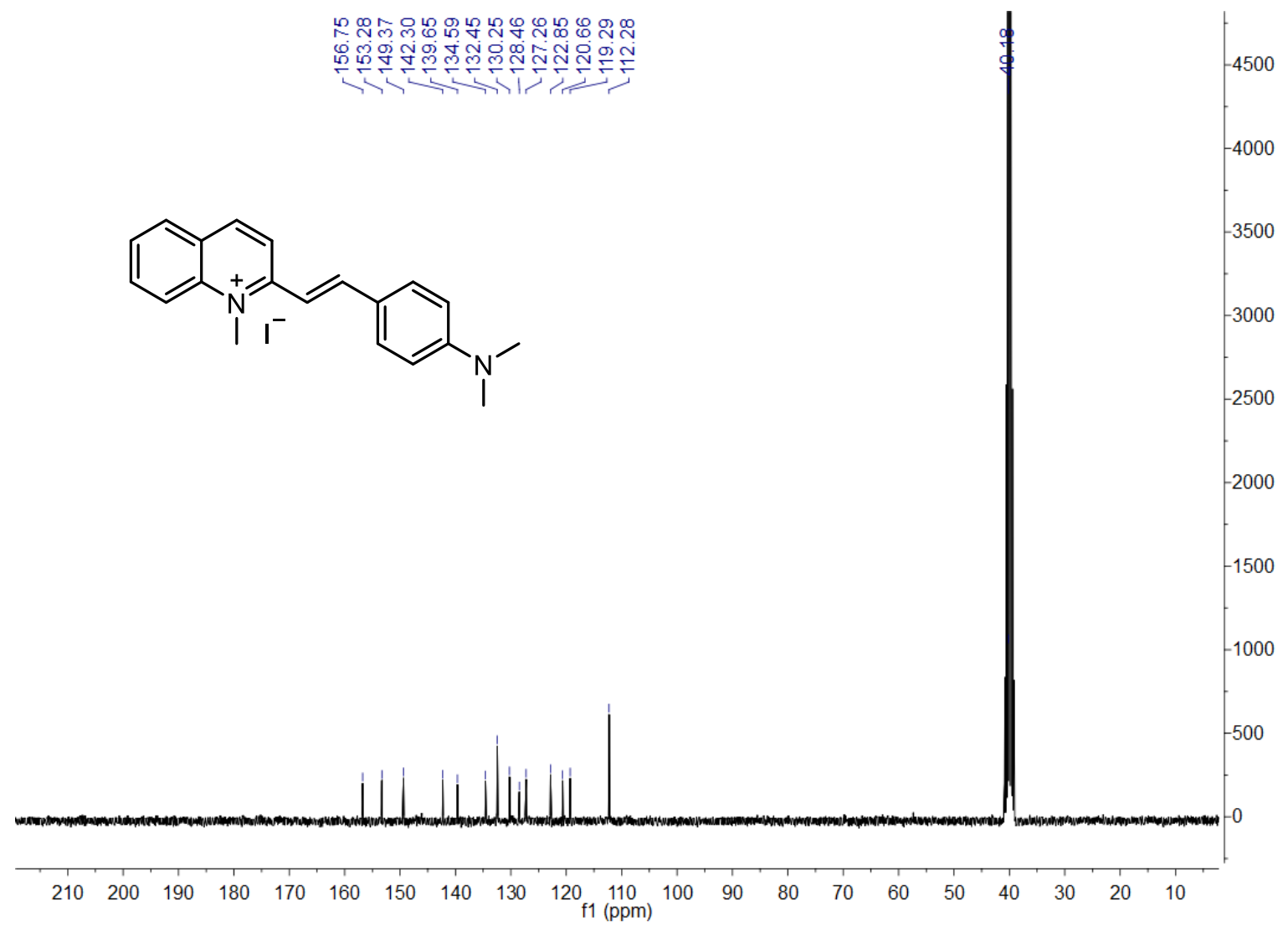

HRMS of compound $\mathbf{2 b}$

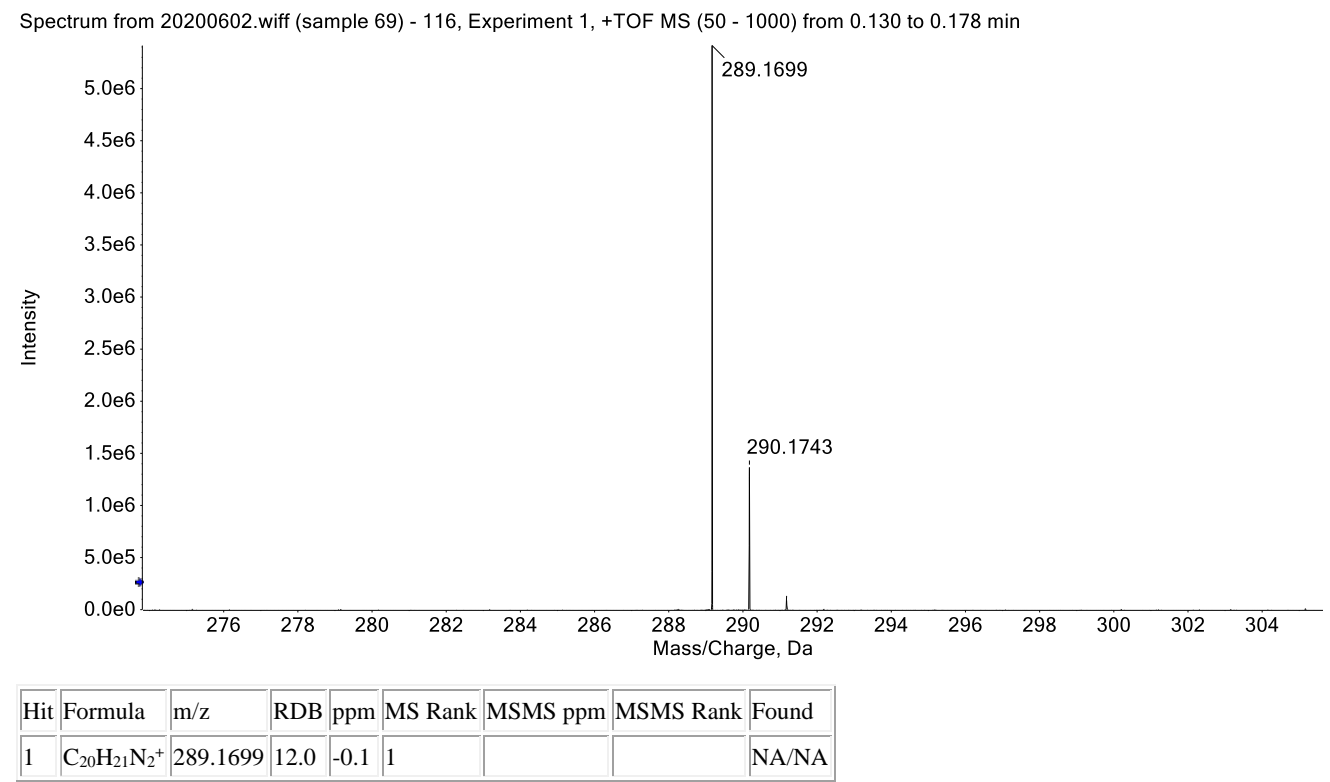

HPLC analysis of $\mathbf{2} \mathbf{b}$ 


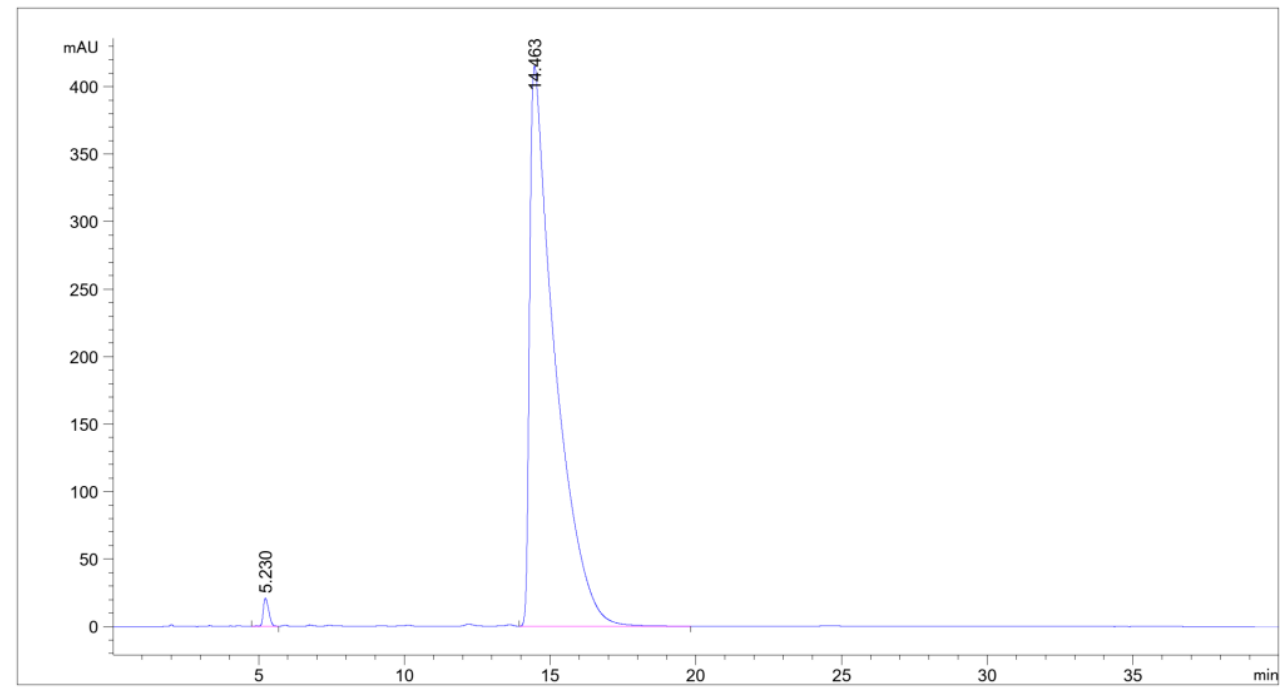

Area Percent Report

$\begin{array}{lll}\text { Sorted By } & : & \text { Signal } \\ \text { Multiplier } & : & 1.0000 \\ \text { Dilution } & : & 1.0000\end{array}$

Use Multiplier \& Dilution Factor with ISTDs

Signal 1: VWD1 A, Wavelength=254 nm

\begin{tabular}{|c|c|c|c|c|c|c|}
\hline $\begin{array}{c}\text { Peak } \\
\text { \# }\end{array}$ & $\begin{array}{c}\text { RetTime } \\
\text { [min] }\end{array}$ & Type & $\begin{array}{l}\text { Width } \\
\text { [min] }\end{array}$ & $\begin{array}{c}\text { Area } \\
{\left[m A U^{\star} s\right]}\end{array}$ & $\begin{array}{l}\text { Height } \\
\text { [mAU] }\end{array}$ & $\begin{array}{c}\text { Area } \\
\frac{\circ}{8}\end{array}$ \\
\hline 1 & 5.230 & $\mathrm{VB} R$ & 0.2099 & 270.74777 & 20.79154 & 1.0713 \\
\hline 2 & 14.463 & $\mathrm{BB}$ & 0.8209 & $2.50022 \mathrm{e} 4$ & 415.00296 & 98.9287 \\
\hline
\end{tabular}

Totals :

$.52729 e 4 \quad 435.79450$

${ }^{1} \mathrm{H}-\mathrm{NMR}$ spectrum of compound $\mathbf{2 c}$ 

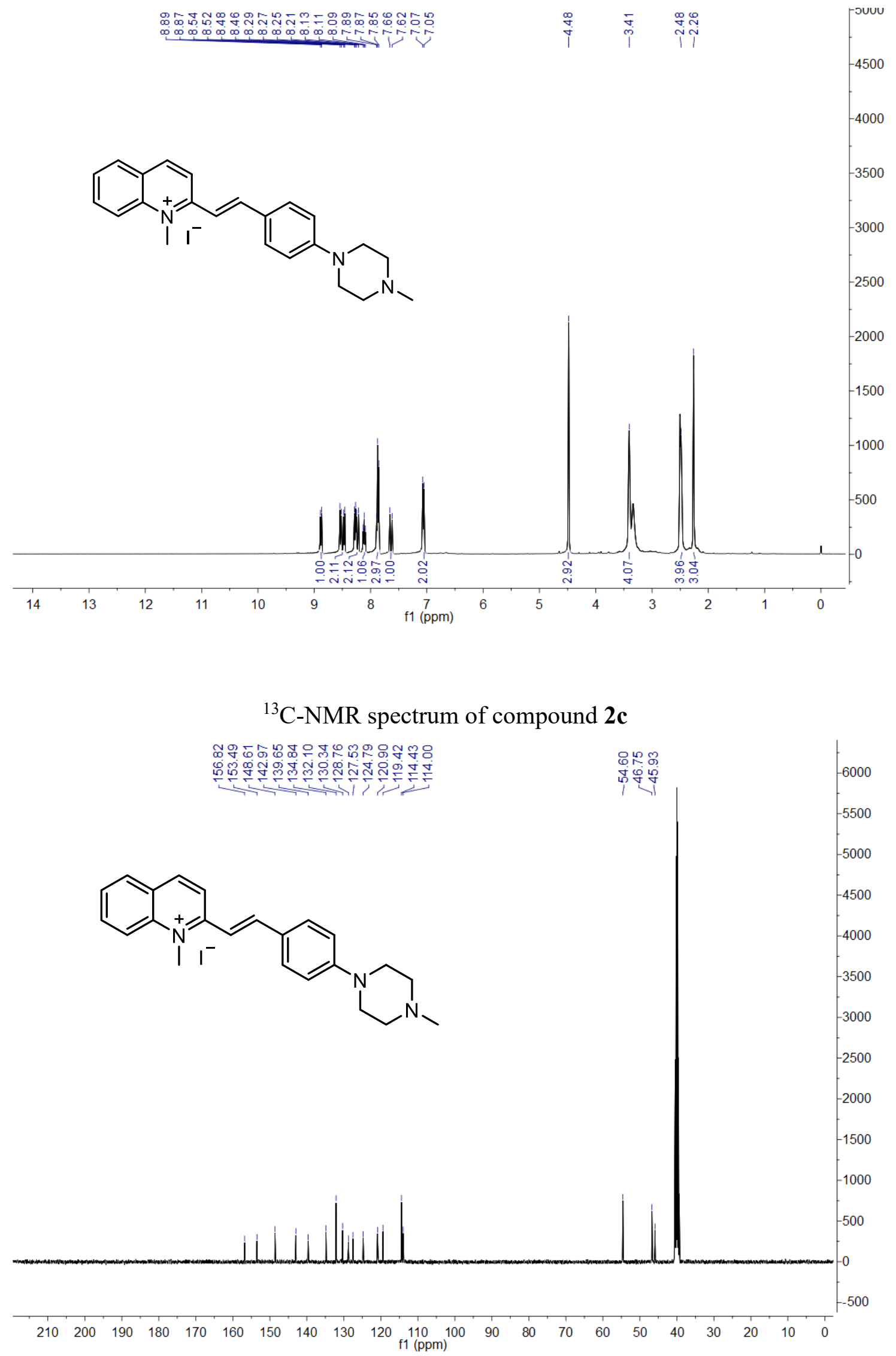

HRMS of compound 2c 


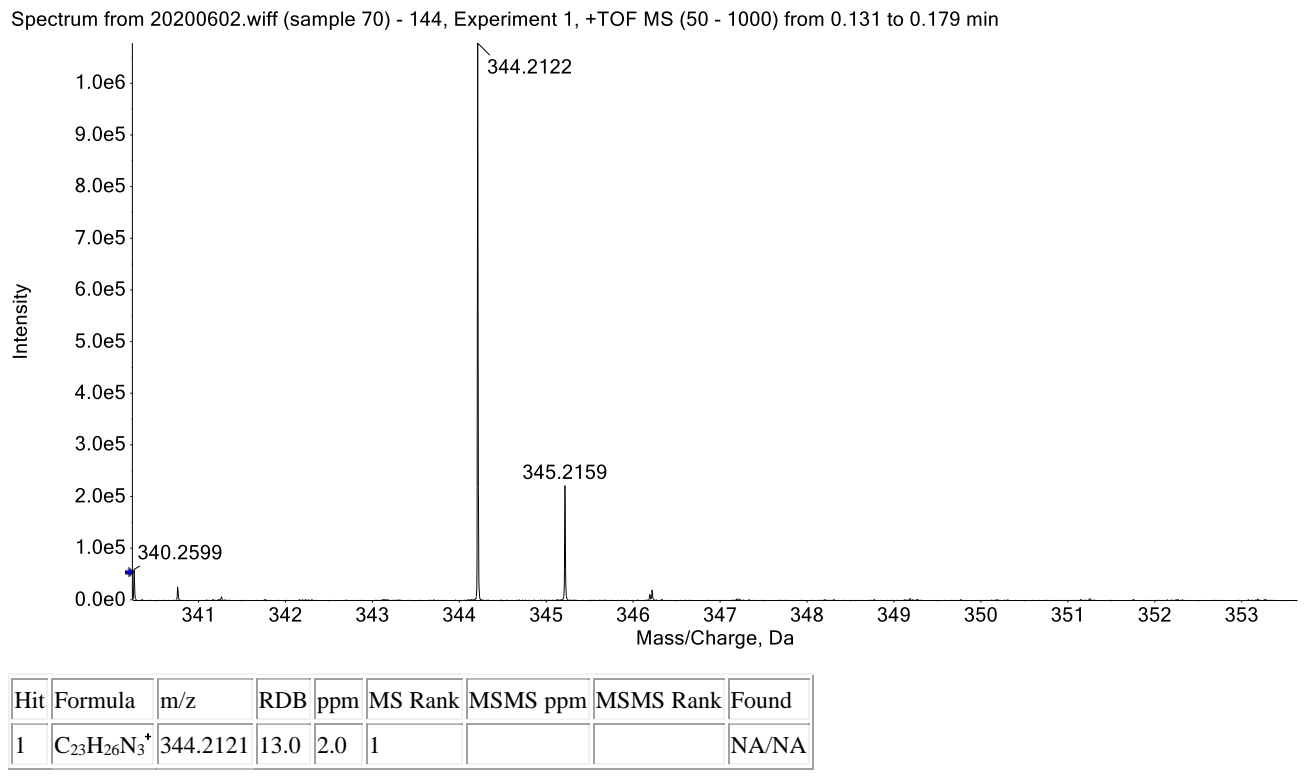

HPLC analysis of $\mathbf{2 c}$

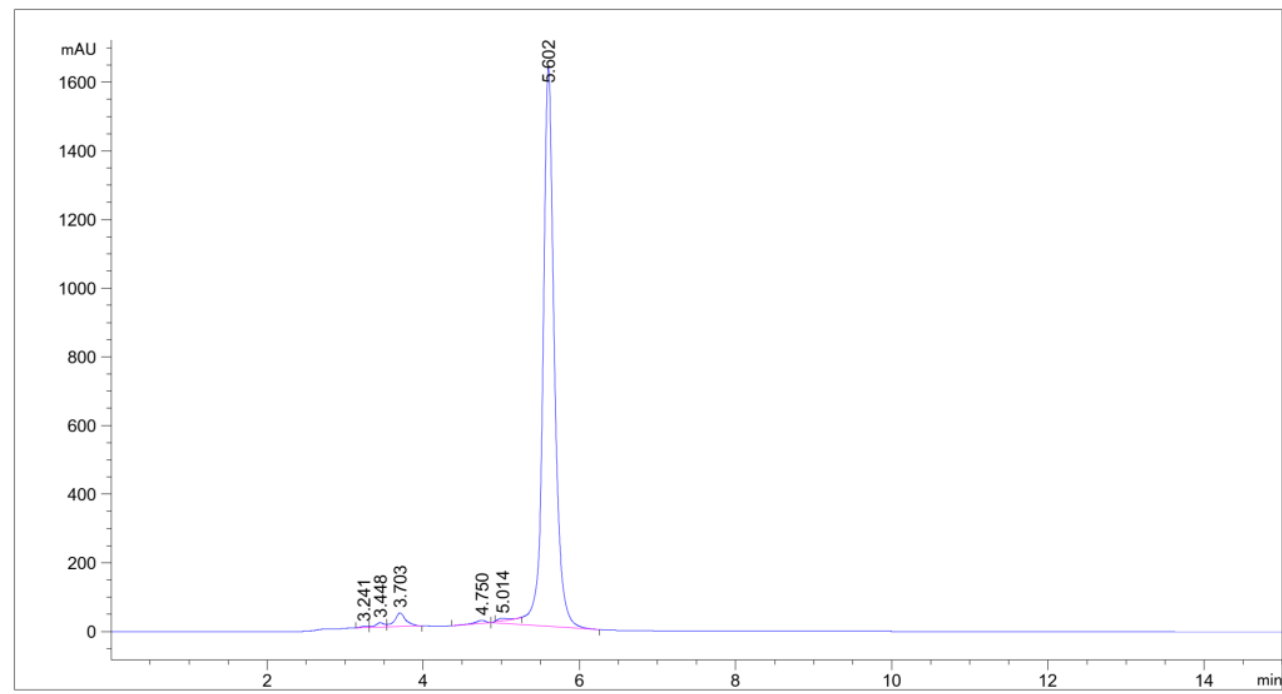

Area Percent Report

\begin{tabular}{|c|c|c|c|c|c|c|}
\hline Sorte & d By & & : & Signal & & \\
\hline Multi & plier & & : & 1.0000 & & \\
\hline Dilut & ion & & : & 1.0000 & & \\
\hline Sampl & e Amount & & & : & 5.00000 & /ul] \\
\hline Use M & lultiplie & er \& & Dilution & Factor with & ISTDs & \\
\hline Signa & 1 : VWD & 11 A, & Waveleng & $\mathrm{gth}=254 \mathrm{~nm}$ & & \\
\hline $\begin{array}{c}\text { Peak } \\
\#\end{array}$ & $\begin{array}{c}\text { RetTime } \\
\text { [min] }\end{array}$ & Type & $\begin{array}{l}\text { Width } \\
\text { [min] }\end{array}$ & $\begin{array}{c}\text { Area } \\
{\left[\mathrm{mAU}^{*} \mathrm{~s}\right]}\end{array}$ & $\begin{array}{l}\text { Height } \\
{[\mathrm{mAU}]}\end{array}$ & $\begin{array}{c}\text { Area } \\
\frac{\circ}{\frac{0}{5}}\end{array}$ \\
\hline & -------1 & --- & $|-------|$ & |---------- & $---------\mid$ & $--------\mid$ \\
\hline 1 & 3.241 & BV & 0.1051 & 22.73903 & 3.35262 & 0.1309 \\
\hline 2 & 3.448 & Vv & 0.1122 & 98.05050 & 12.68636 & 0.5643 \\
\hline 3 & 3.703 & VB & 0.1414 & 395.50122 & 39.65481 & 2.2764 \\
\hline 4 & 4.750 & BB & 0.1485 & 87.54816 & 8.83980 & 0.5039 \\
\hline 5 & 5.014 & BV E & 0.1406 & 60.13360 & 6.90519 & 0.3461 \\
\hline 6 & 5.602 & VB R & 0.1528 & $1.67102 \mathrm{e} 4$ & 1627.20361 & 96.1784 \\
\hline
\end{tabular}

${ }^{1} \mathrm{H}-\mathrm{NMR}$ spectrum of compound $\mathbf{2 d}$ 

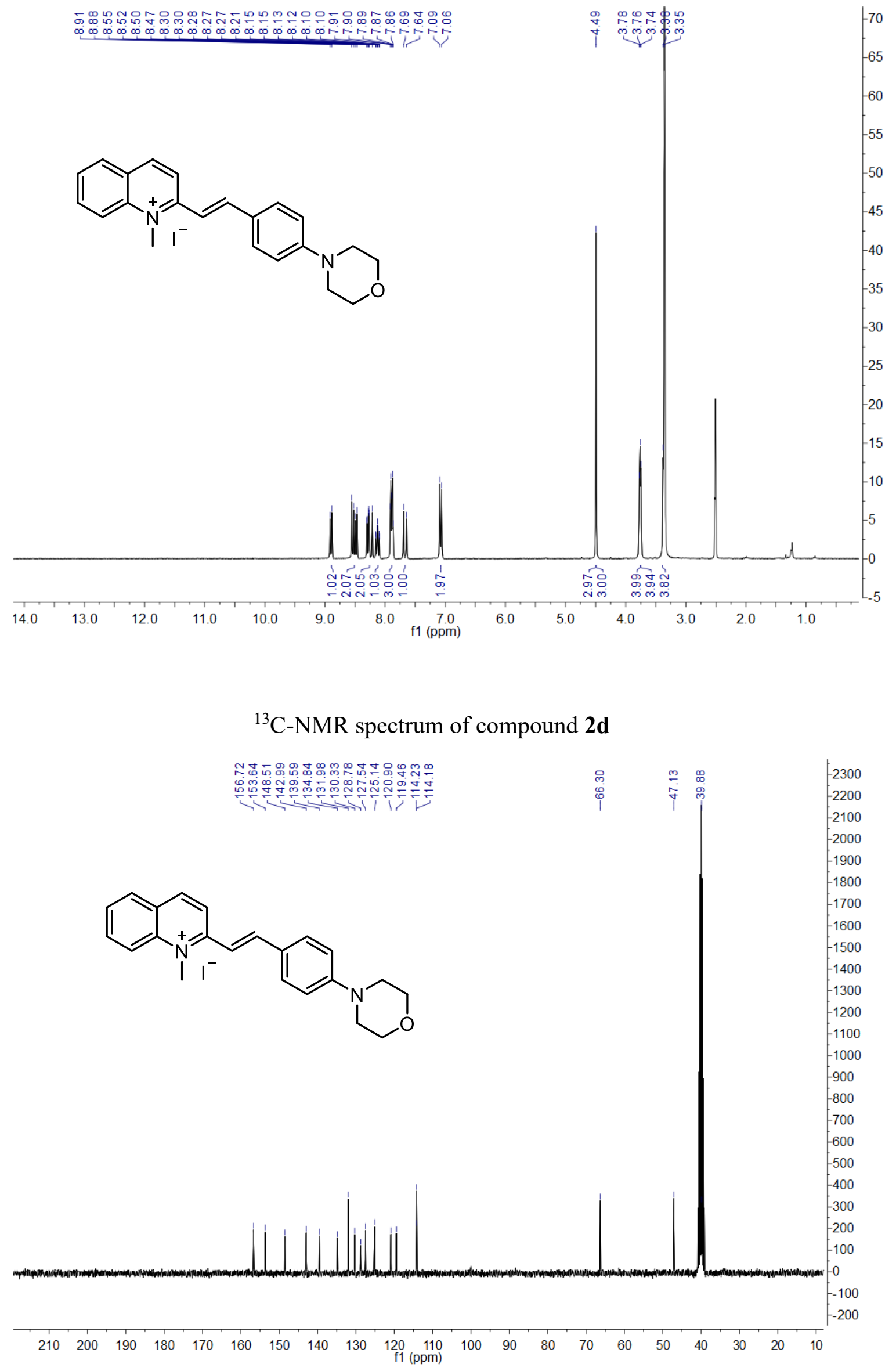
HRMS of compound 2d

Spectrum from 20200602.wiff (sample 71) - 117, Experiment 1, +TOF MS (50 - 1000) from 0.129 to $0.177 \mathrm{~min}$

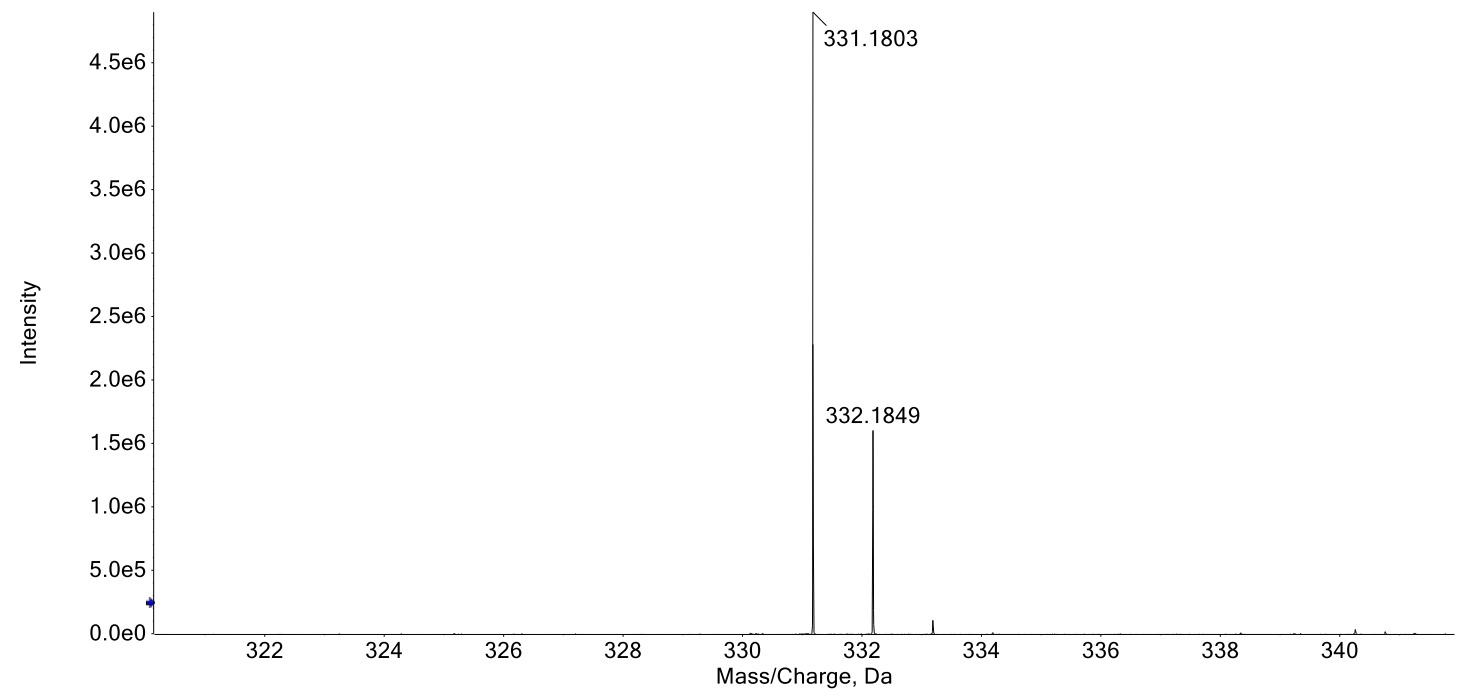

$\begin{array}{lllllll}\text { Hit Formula } & \mathrm{m} / \mathrm{z} & \text { RDB } & \mathrm{ppm} & \text { MS Rank MSMS ppm MSMS Rank Found }\end{array}$

\begin{tabular}{|l|l|l|l|l|l|l|}
\hline 1 & $\mathrm{C}_{22} \mathrm{H}_{23} \mathrm{~N}_{2} \mathrm{O}^{+}$ & 331.1805 & 13.0 & -0.6 & 1 & \\
\hline
\end{tabular}

HPLC analysis of $\mathbf{2 d}$ 


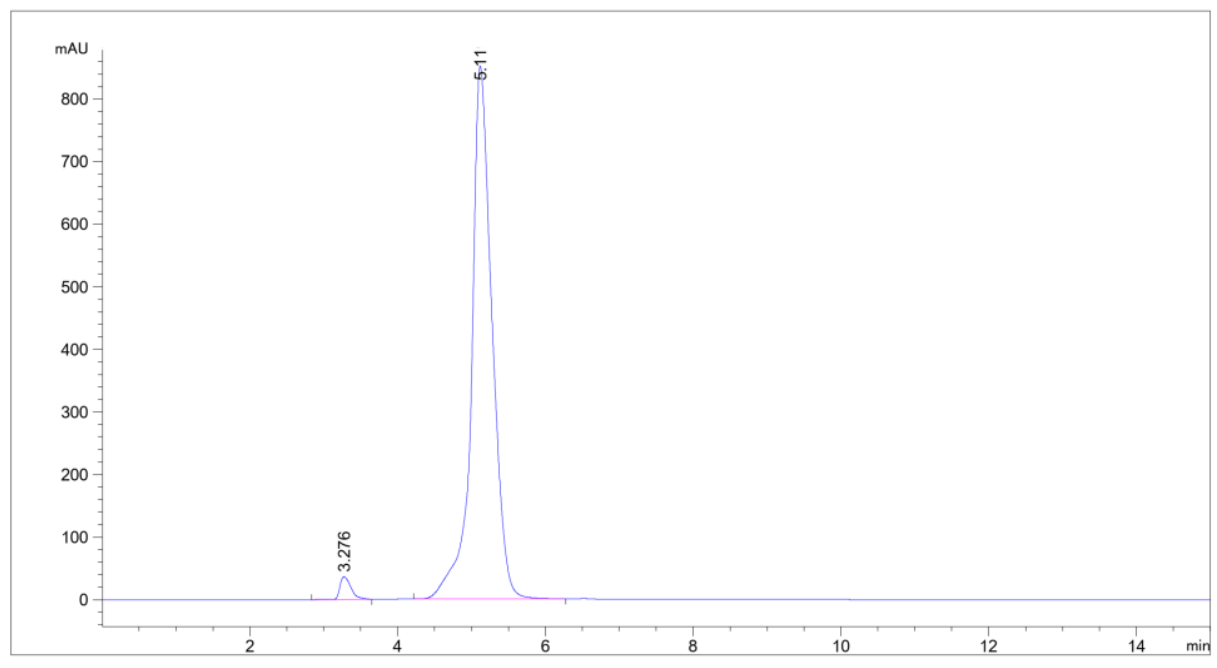

Area Percent Report

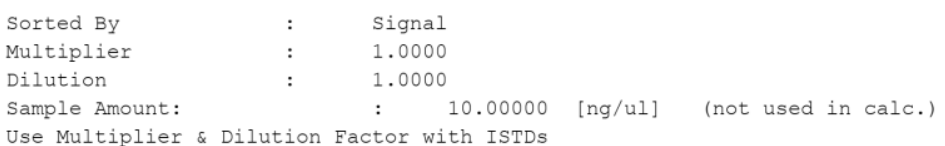

Use Multiplier \& Dilution Factor with ISTDs

Signal 1: VWD1 A, Wavelengt $h=250 \mathrm{~nm}$

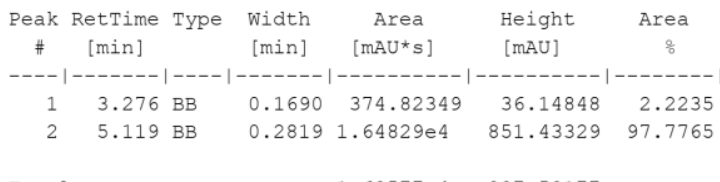

Totals : $\quad 1.68577 e^{4} \quad 887.58177$

${ }^{1} \mathrm{H}-\mathrm{NMR}$ spectrum of compound $\mathbf{2 e}$

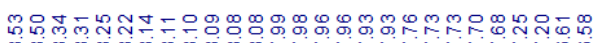

舟

$\underset{\sim}{\stackrel{\sim}{*}}$

(c)

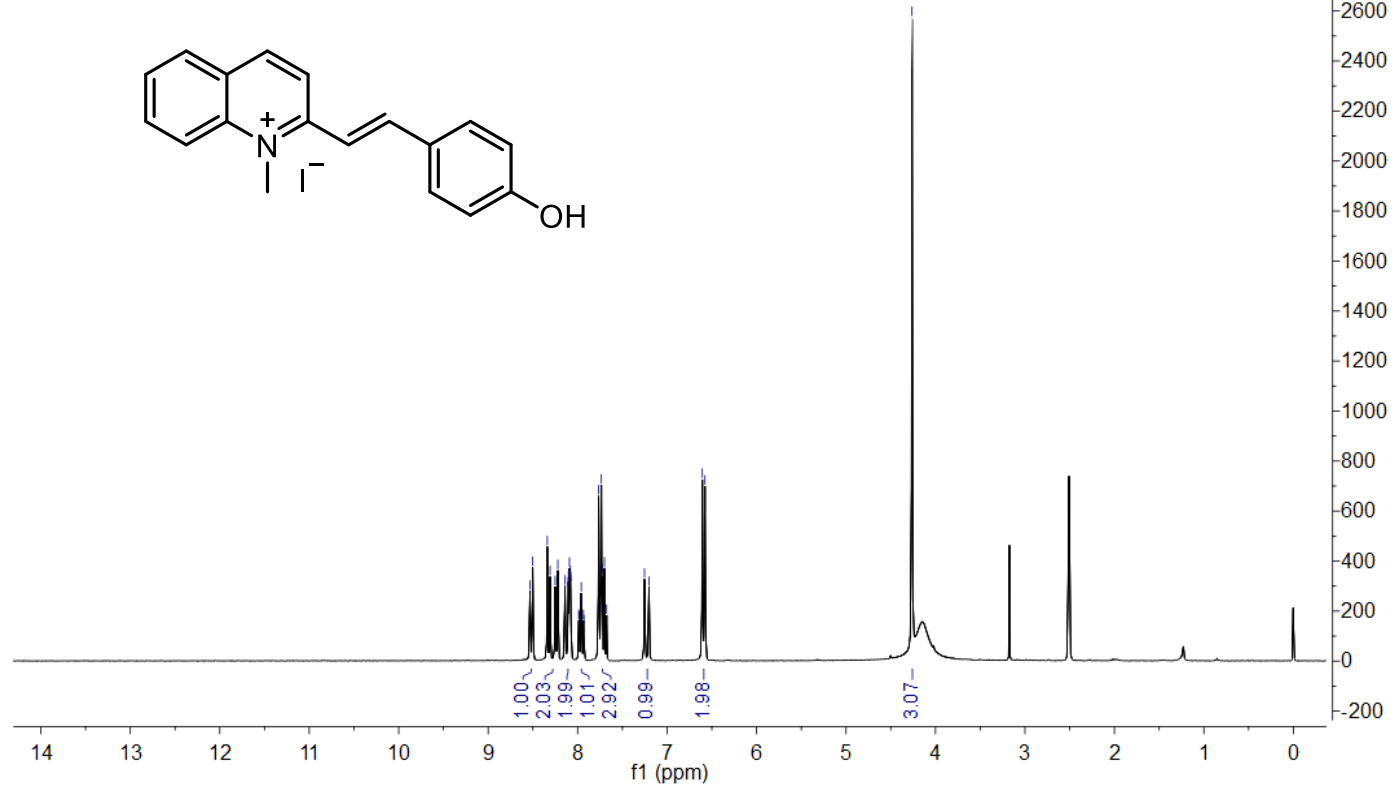




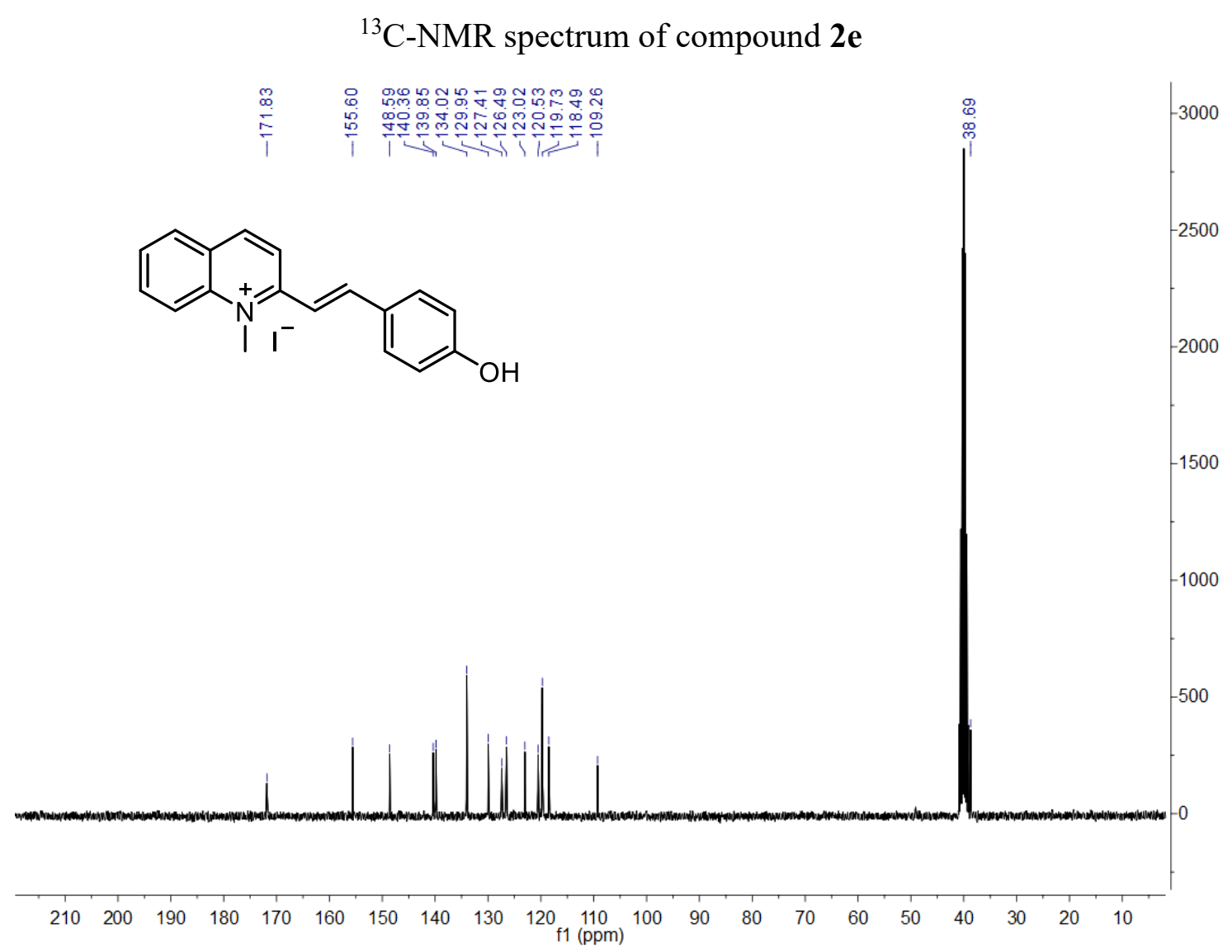

HRMS of compound $\mathbf{2 e}$

Spectrum from 20200602.wiff (sample 73) - 124, Experiment 1, +TOF MS (50 - 1000) from 0.130 to $0.178 \mathrm{~min}$

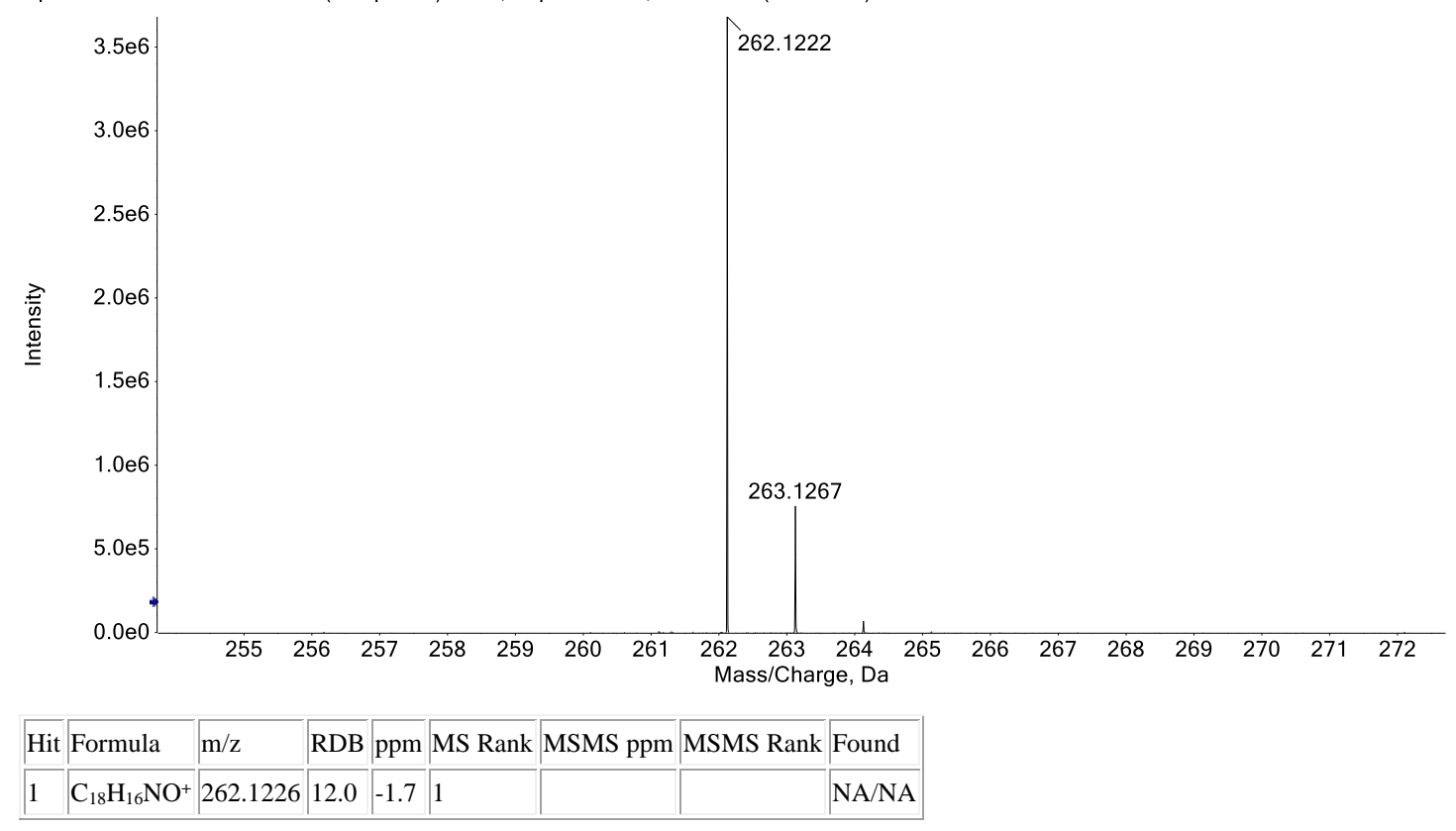

HPLC analysis of $\mathbf{2 e}$ 


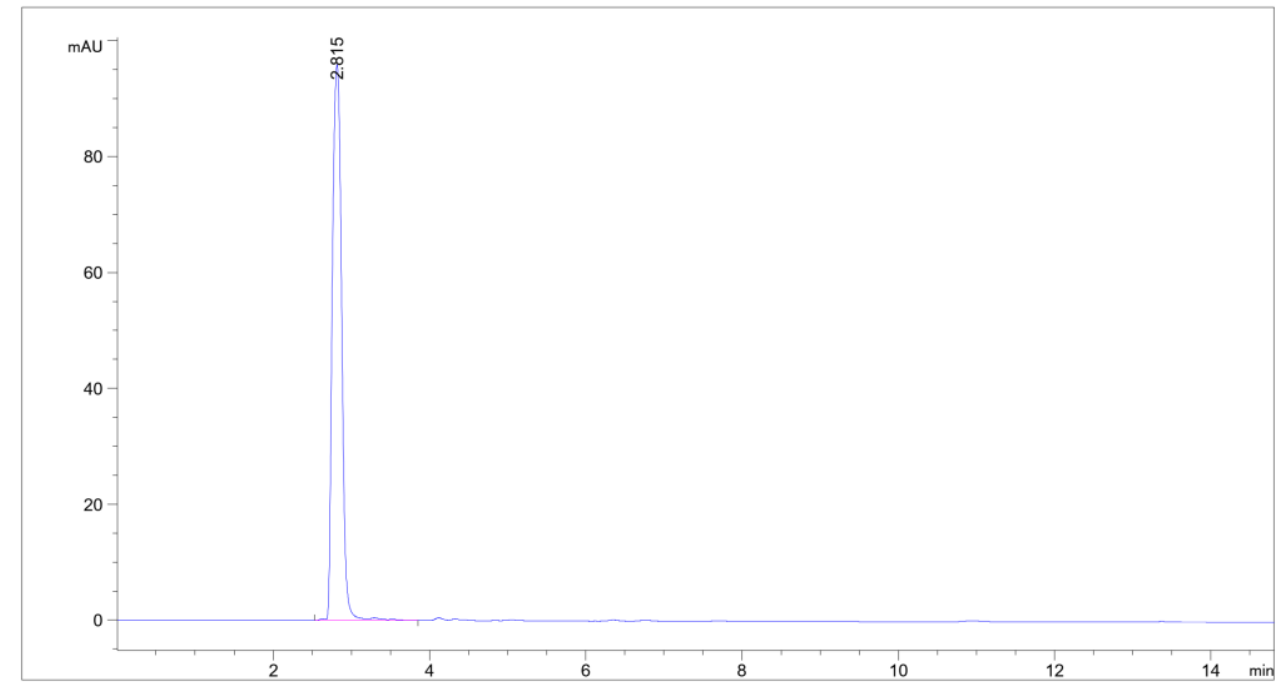

Area Percent Report

$\begin{array}{llll}\text { Sorted By } & : & \text { Signal } & \\ \text { Multiplier } & : & 1.0000 & \\ \text { Dilution } & : & 1.0000 & \\ \text { Sample Amount: } & & : \quad 10.00000 \quad \text { [ng/ul] } \quad \text { (not used in calc.) }\end{array}$

Use Multiplier \& Dilution Factor with ISTDs

Signal 1: VWD1 A, Wavelength $=254 \mathrm{~nm}$

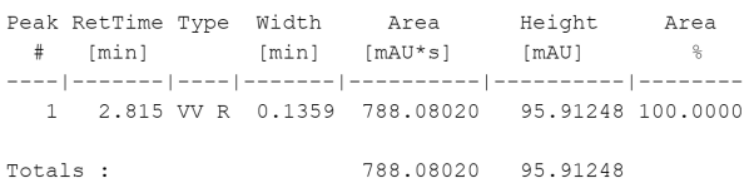

${ }^{1} \mathrm{H}-\mathrm{NMR}$ spectrum of compound $\mathbf{2 f}$ 

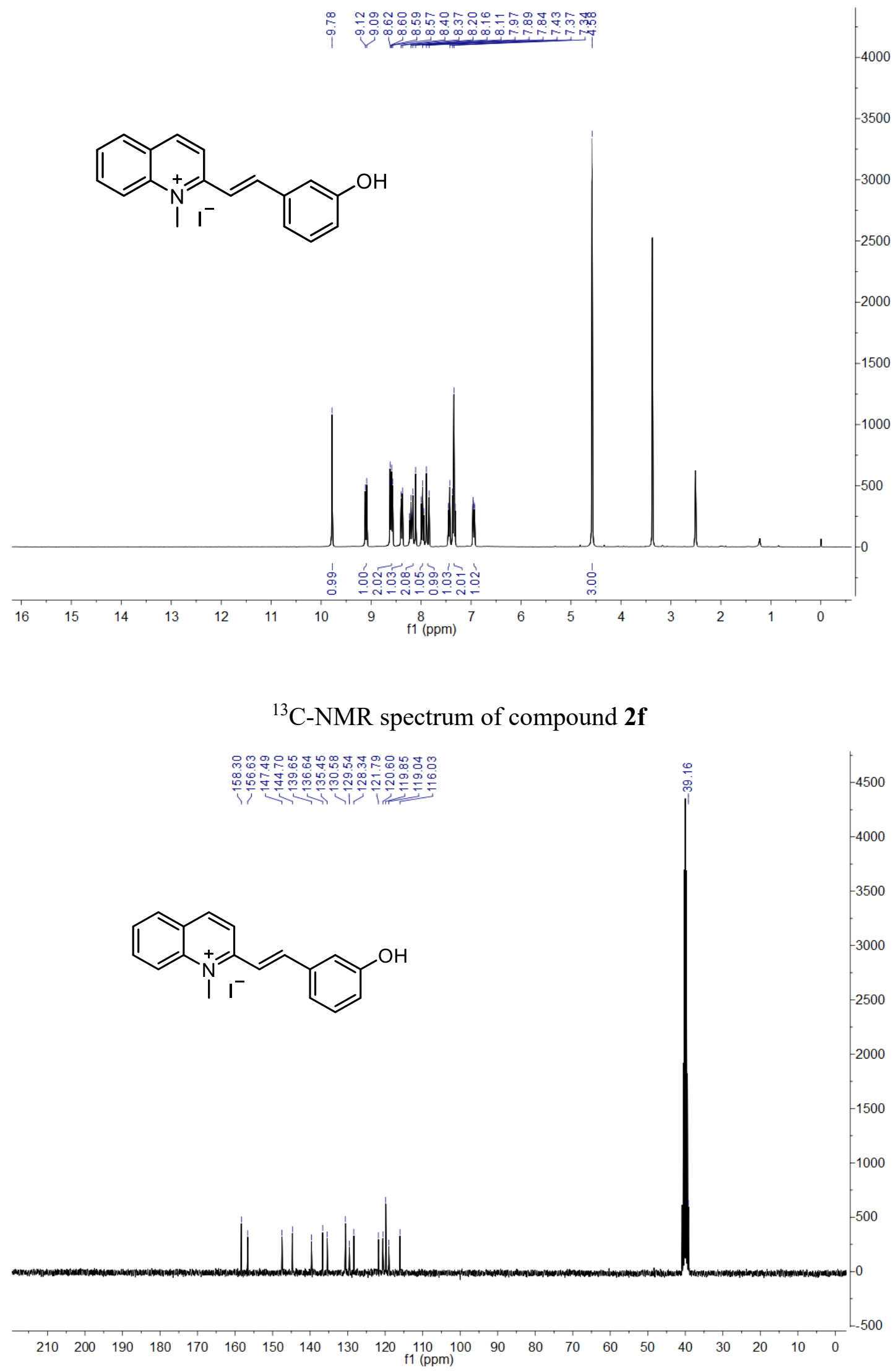

HRMS of compound $\mathbf{2 f}$ 
Spectrum from 20200602.wiff (sample 72) - 151, Experiment 1, +TOF MS (50 - 1000) from 0.130 to $0.178 \mathrm{~min}$

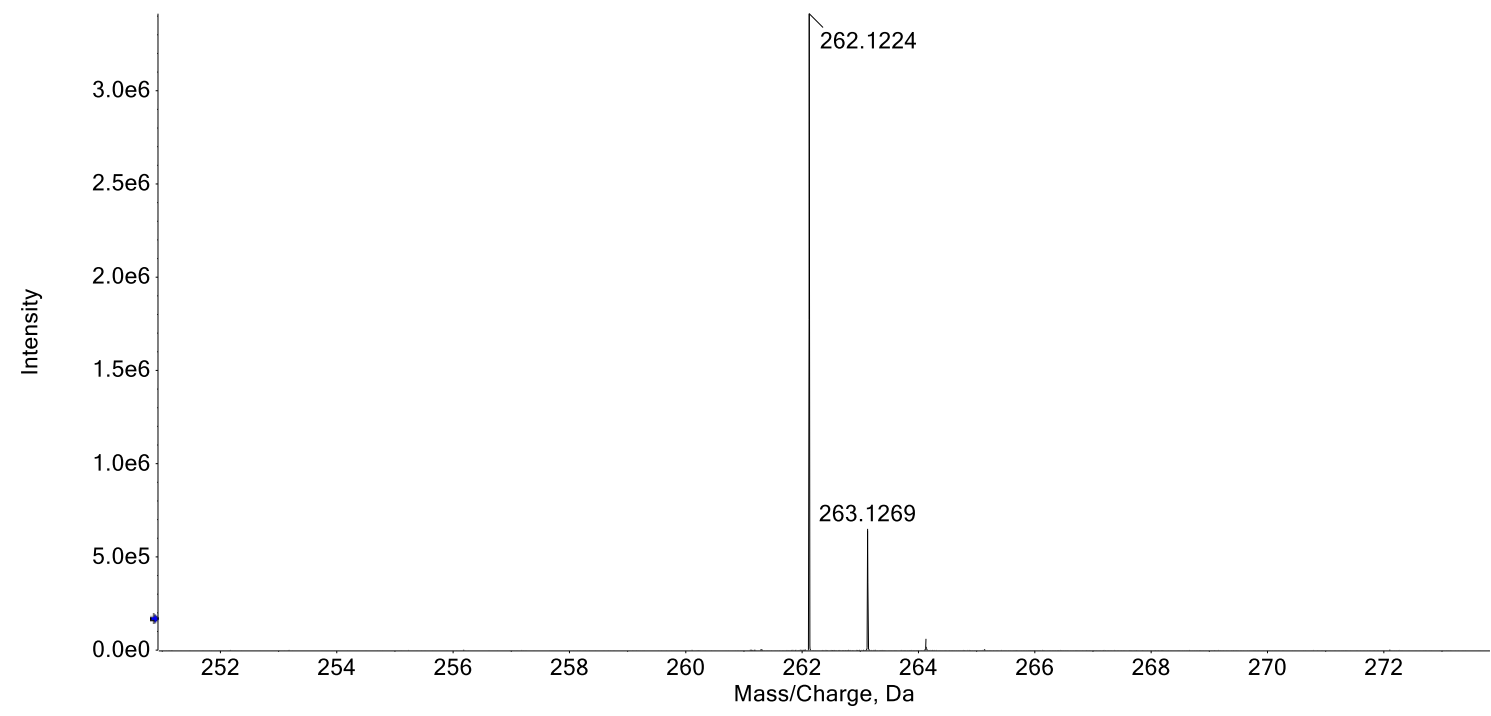

$\begin{array}{llll}\text { Hit Formula } & \mathrm{m} / \mathrm{z} & \text { RDB } & \text { ppm MS Rank MSMS ppm MSMS Rank Found }\end{array}$

\begin{tabular}{|l|l|l|l|l|l|l|l|}
\hline 1 & $\mathrm{C}_{18} \mathrm{H}_{16} \mathrm{NO}^{+}$ & 262.1226 & 12.0 & -0.9 & 1 & & NA/NA \\
\hline
\end{tabular}

\section{HPLC analysis of $\mathbf{2 f}$}

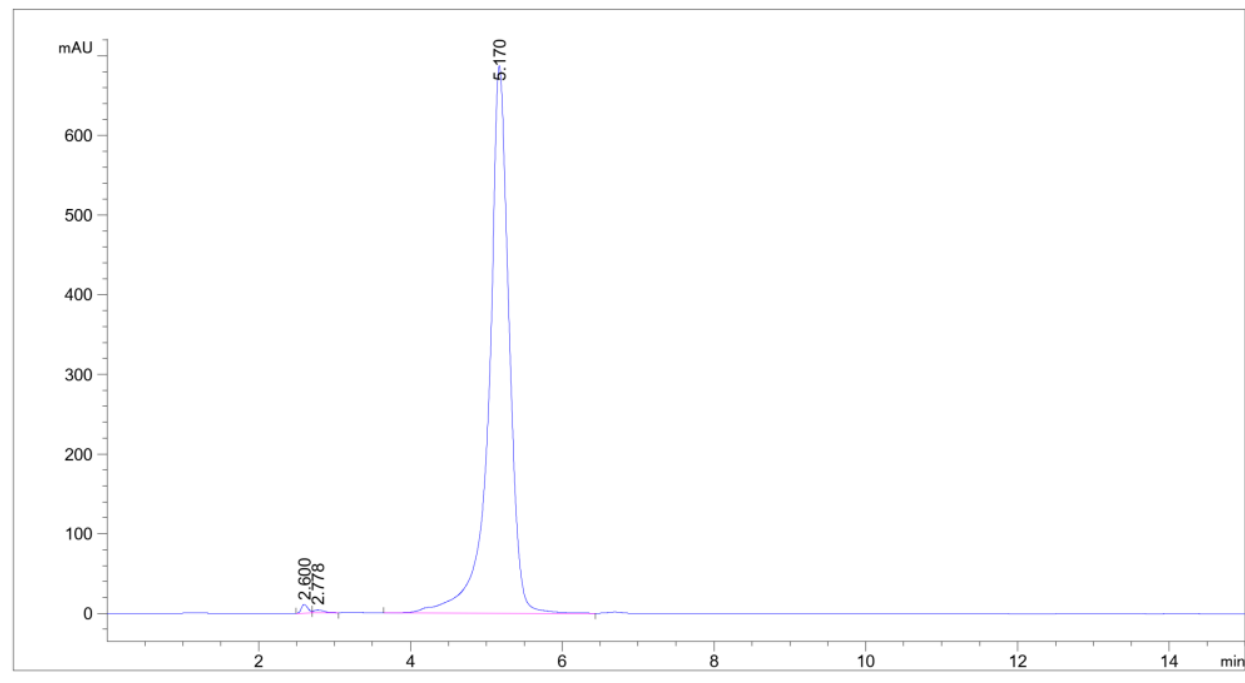

Area Percent Report

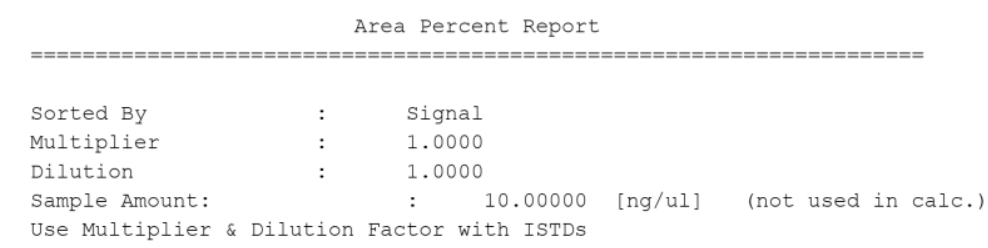

Signal 1: VWD1 A, Wavelength=254 nm

\begin{tabular}{|c|c|c|c|c|c|c|}
\hline $\begin{array}{c}\text { Peak } \\
\#\end{array}$ & $\begin{array}{c}\text { RetTime } \\
\text { [min] }\end{array}$ & Type & $\begin{array}{l}\text { Width } \\
\text { [min] }\end{array}$ & $\begin{array}{c}\text { Area } \\
{\left[\mathrm{mAU}{ }^{*} \mathrm{~s}\right]}\end{array}$ & $\begin{array}{l}\text { Height } \\
\text { [mAU] }\end{array}$ & $\begin{array}{c}\text { Area } \\
\frac{\circ}{0}\end{array}$ \\
\hline 1 & 2.600 & BV & 0.0950 & 66.06271 & 10.86080 & 0.5175 \\
\hline 2 & 2.778 & VB & 0.1524 & 35.91490 & 3.72633 & 0.2813 \\
\hline 3 & 5.170 & BB & 0.2661 & $1.26646 \mathrm{e} 4$ & 687.02161 & 99.2012 \\
\hline
\end{tabular}

Totals : $\quad 1.27666 \mathrm{e} 4 \quad 701.60874$ 

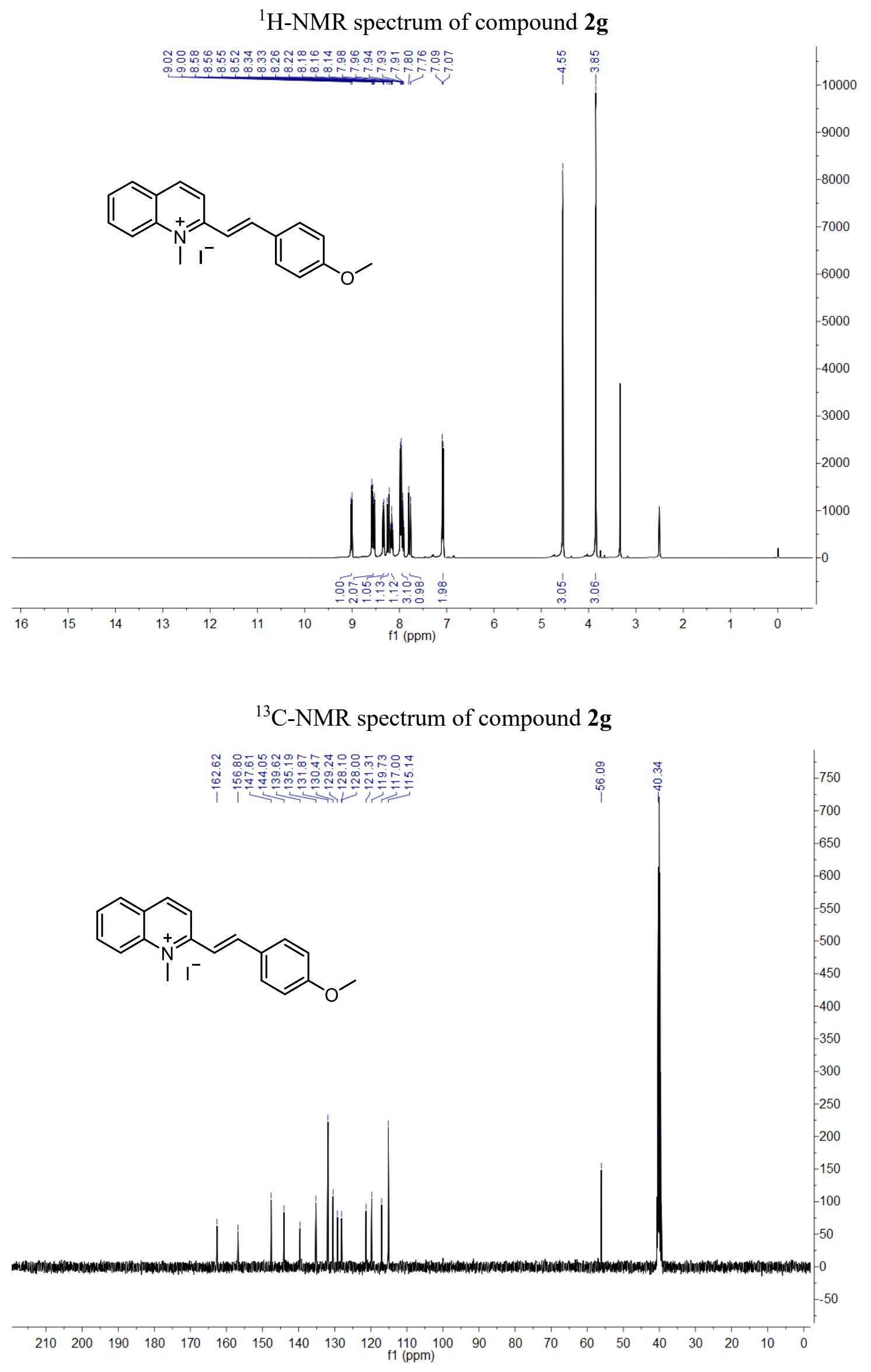
HRMS of compound $\mathbf{2 g}$

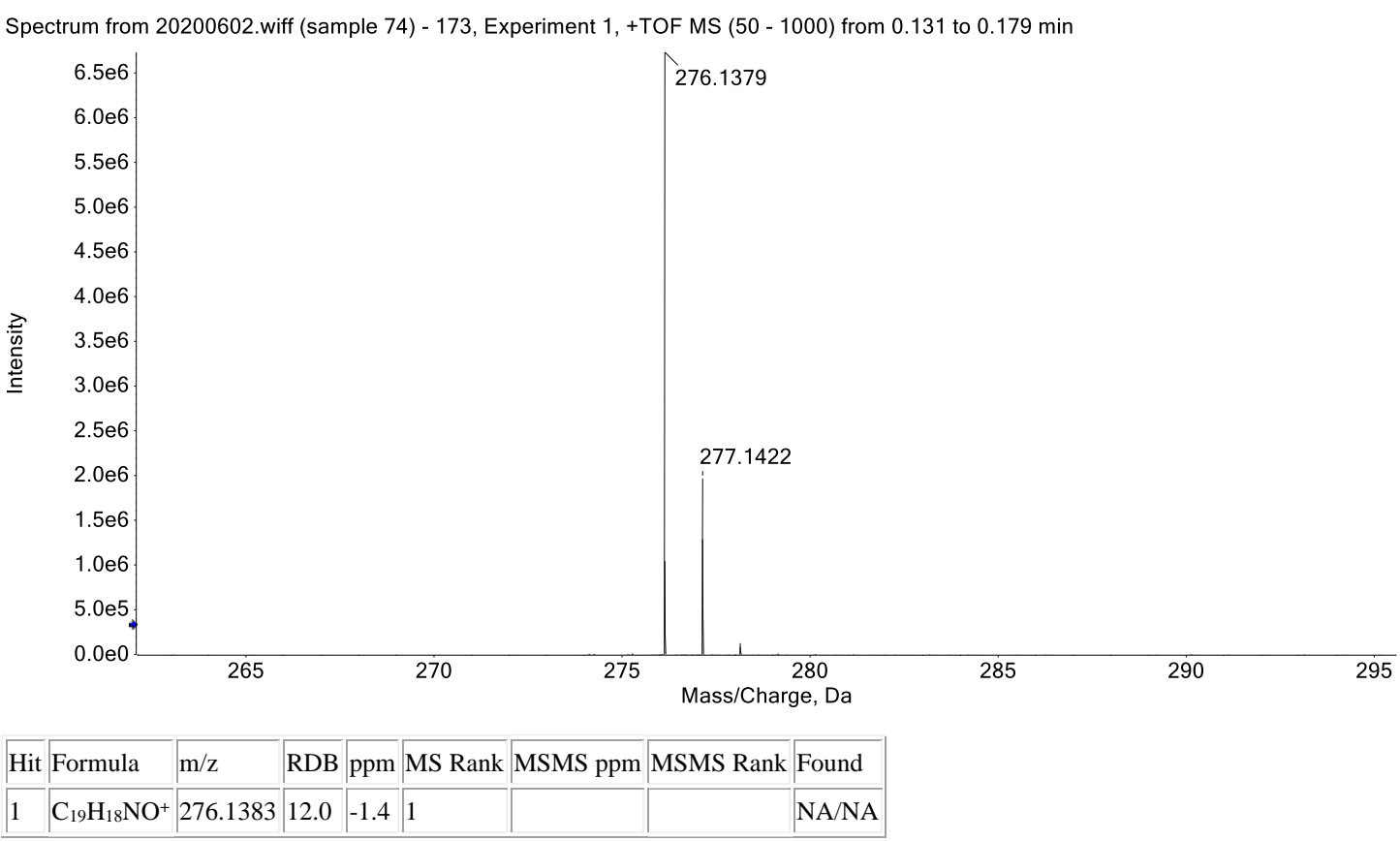

HPLC analysis of $\mathbf{2 g}$ 


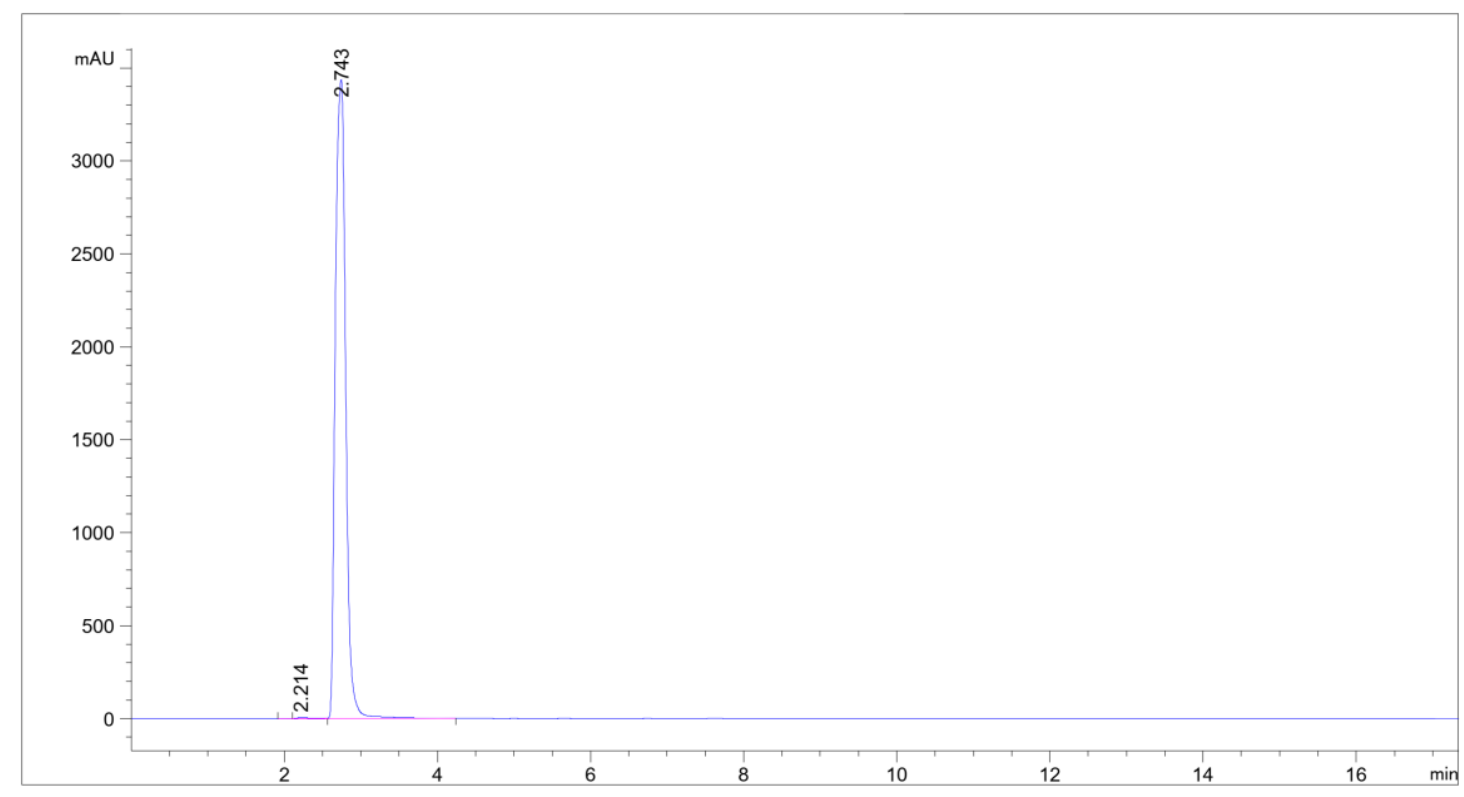

Area Percent Report

\begin{tabular}{|c|c|c|c|c|}
\hline Sorted By & : & Signal & & \\
\hline Multiplier & : & 1.0000 & & \\
\hline Dilution & : & 1.0000 & & \\
\hline Sample Amount: & & $: \quad 10.00000$ & [ng/ul] & (not used in calc.) \\
\hline
\end{tabular}

Signal 1: VWD1 A, Wavelength=250 nm

\begin{tabular}{|c|c|c|c|c|c|c|}
\hline $\begin{array}{l}\text { Peak } \\
\quad \#\end{array}$ & $\begin{array}{c}\text { RetTime } \\
\text { [min] }\end{array}$ & Type & $\begin{array}{l}\text { Width } \\
\text { [min] }\end{array}$ & $\begin{array}{c}\text { Area } \\
{\left[\mathrm{mAU}^{\star} \mathrm{s}\right]}\end{array}$ & $\begin{array}{l}\text { Height } \\
\text { [mAU] }\end{array}$ & $\begin{array}{c}\text { Area } \\
\frac{\circ}{0}\end{array}$ \\
\hline \multicolumn{7}{|c|}{$----|-------|----|-------|----------|----------|--------$} \\
\hline 1 & 2.214 & VV E & 0.1363 & 56.96567 & 5.61607 & 0.1756 \\
\hline 2 & 2.743 & VV R & 0.1510 & $3.23851 e 4$ & 3435.85303 & 99.8244 \\
\hline & : & & & $3.24421 e 4$ & 3441.46909 & \\
\hline
\end{tabular}

${ }^{1}$ H-NMR spectrum of compound $\mathbf{2 h}$ 


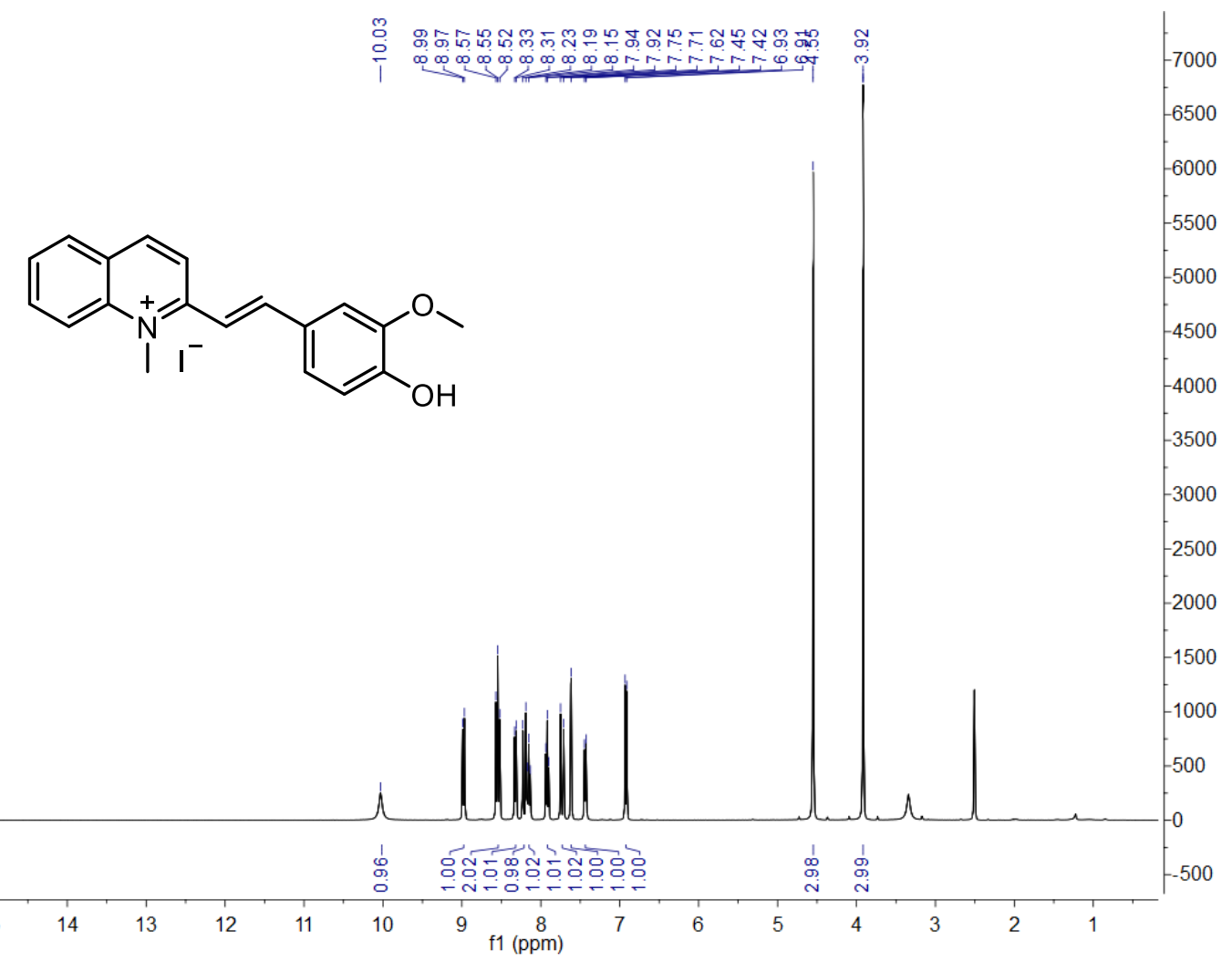

${ }^{13} \mathrm{C}-\mathrm{NMR}$ spectrum of compound $\mathbf{2 h}$

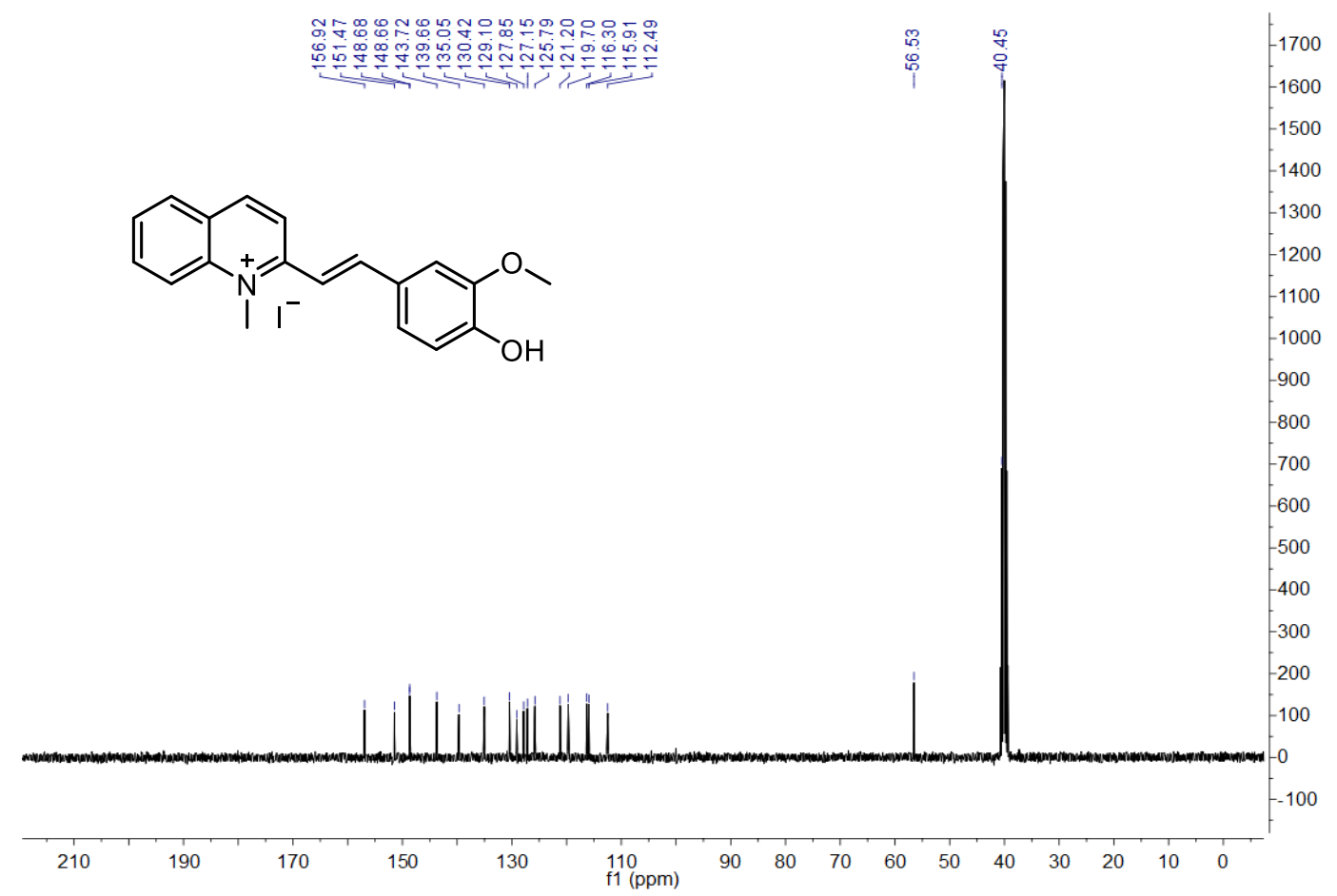

HRMS of compound $\mathbf{2 h}$ 
Spectrum from 20200602.wiff (sample 75) - 170, Experiment 1, +TOF MS (50 - 1000) from 0.130 to 0.178 min

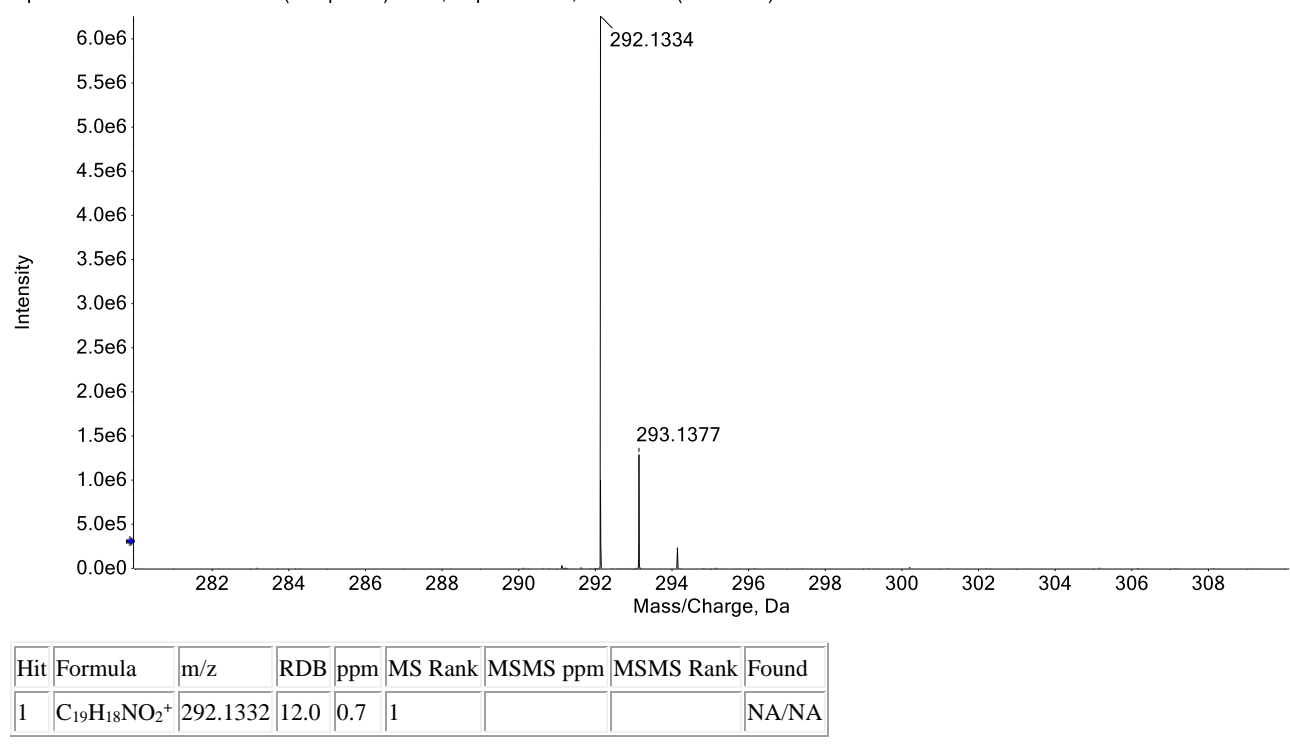

HPLC analysis of $\mathbf{2 h}$

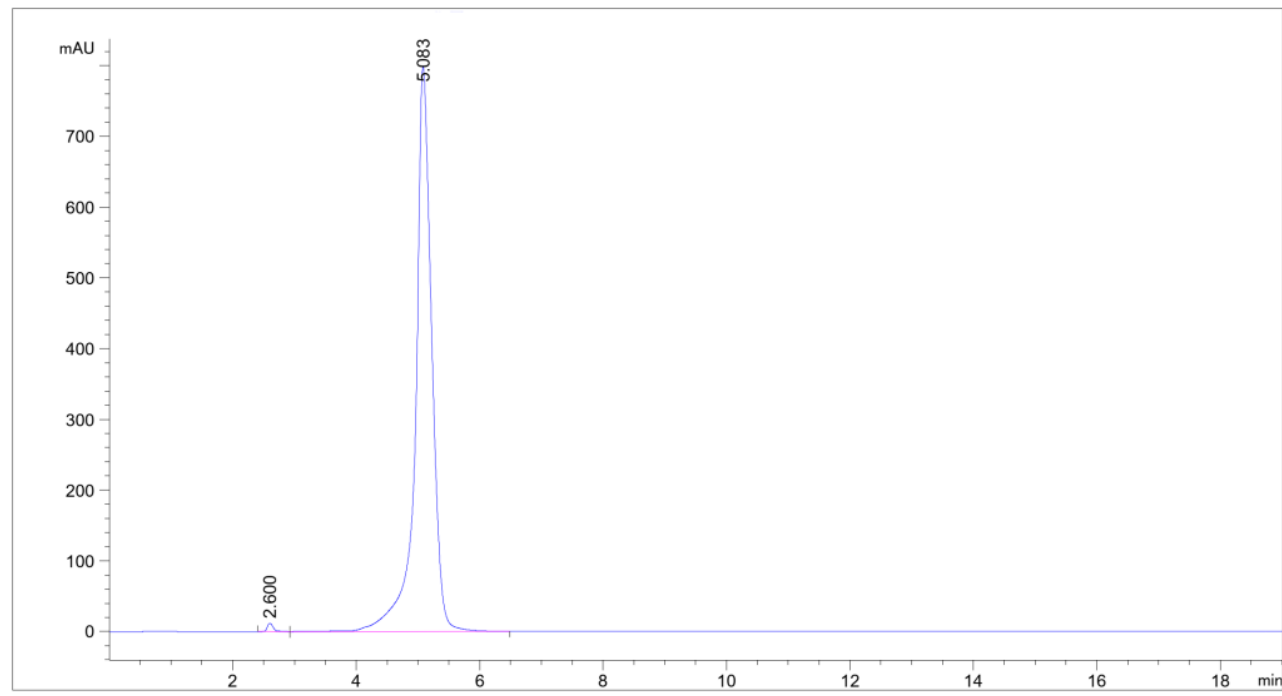

Area Percent Report

$\begin{array}{lccc}\text { Sorted By } & : & \text { Signal } & \\ \text { Multiplier } & : & 1.0000 & \\ \text { Dilution } & : & 1.0000 & \\ \text { Sample Amount: } & & : \quad 10.00000 \quad \text { [ng/ul] } \quad \text { (not used in calc.) } \\ \text { Use Multiplier \& Dilution Factor with ISTDs } & \end{array}$

Signal 1: VWD1 A, Wavelength=254 nm

\begin{tabular}{|c|c|c|c|c|c|c|}
\hline $\begin{array}{c}\text { Peak } \\
\quad \#\end{array}$ & $\begin{array}{c}\text { RetTime } \\
\text { [min] }\end{array}$ & Type & $\begin{array}{l}\text { Width } \\
\text { [min] }\end{array}$ & $\begin{array}{c}\text { Area } \\
{[\mathrm{mAU} * \mathrm{~s}]}\end{array}$ & $\begin{array}{l}\text { Height } \\
\text { [MAU] }\end{array}$ & $\begin{array}{c}\text { Area } \\
\frac{\%}{0}\end{array}$ \\
\hline & & & & |--------- & ---.--- & ------ \\
\hline 1 & 2.600 & BB & 0.1089 & 81.68074 & 11.63140 & 0.5542 \\
\hline 2 & 5.083 & VB $R$ & 0.2652 & $1.46561 \mathrm{e} 4$ & 798.08807 & 99.4458 \\
\hline Total & & & & $1.47378 \mathrm{e} 4$ & 809.71948 & \\
\hline
\end{tabular}


${ }^{1} \mathrm{H}-\mathrm{NMR}$ spectrum of compound $\mathbf{2 i}$

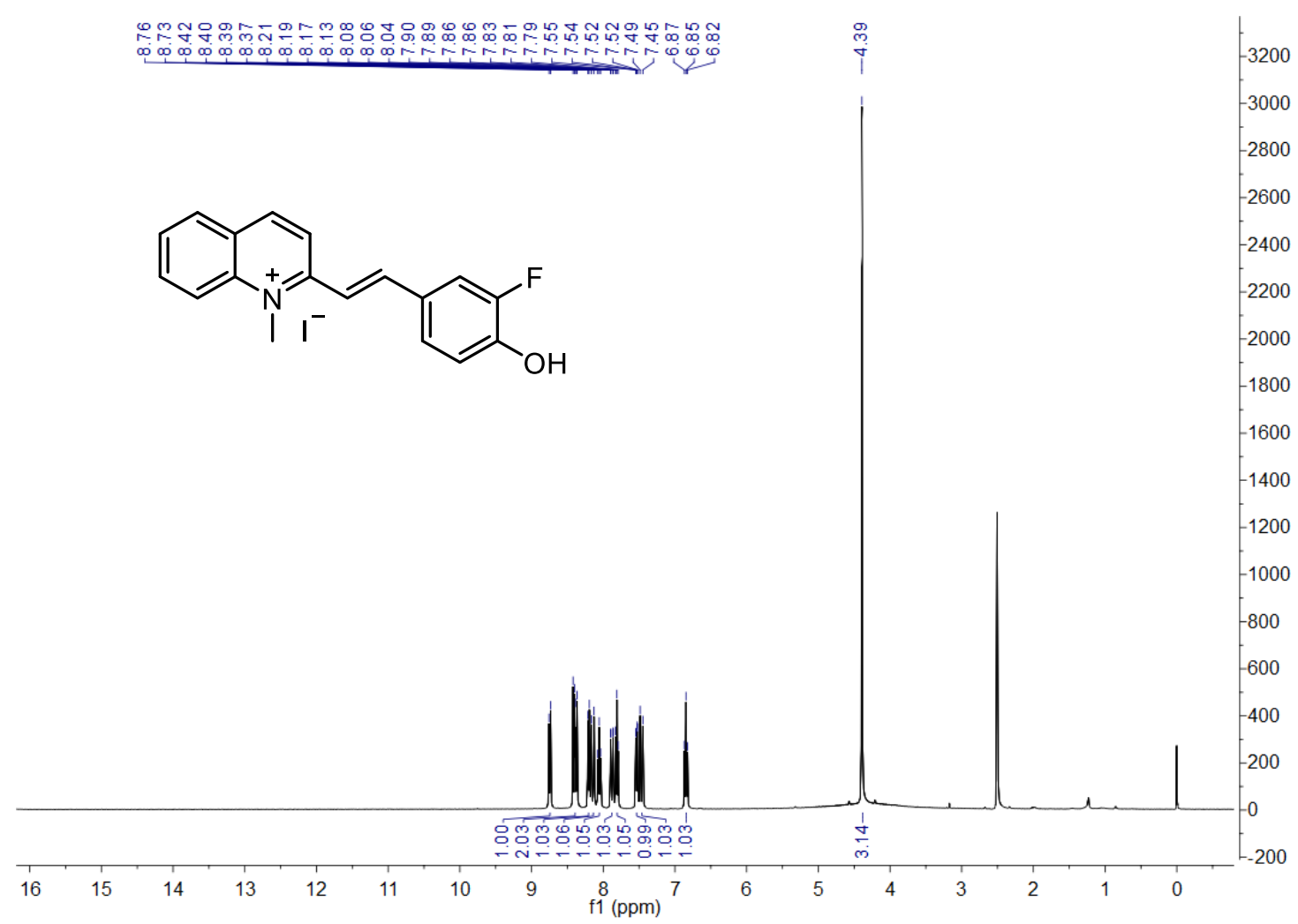

${ }^{13} \mathrm{C}-\mathrm{NMR}$ spectrum of compound $\mathbf{2 i}$

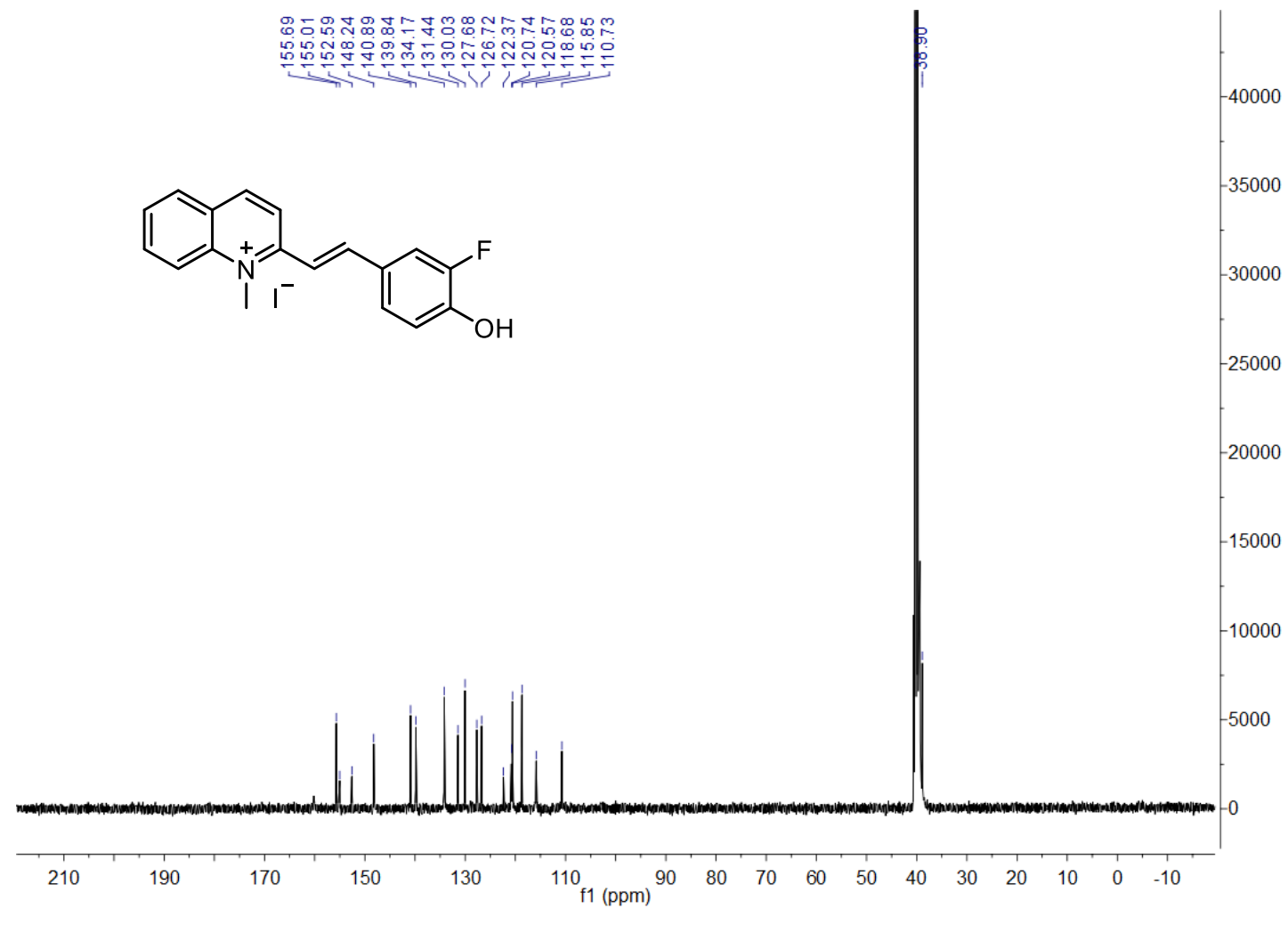

HRMS of compound $\mathbf{2 i}$ 
Spectrum from 20200602.wiff (sample 76) - 168, Experiment 1, +TOF MS (50 - 1000) from 0.130 to $0.178 \mathrm{~min}$

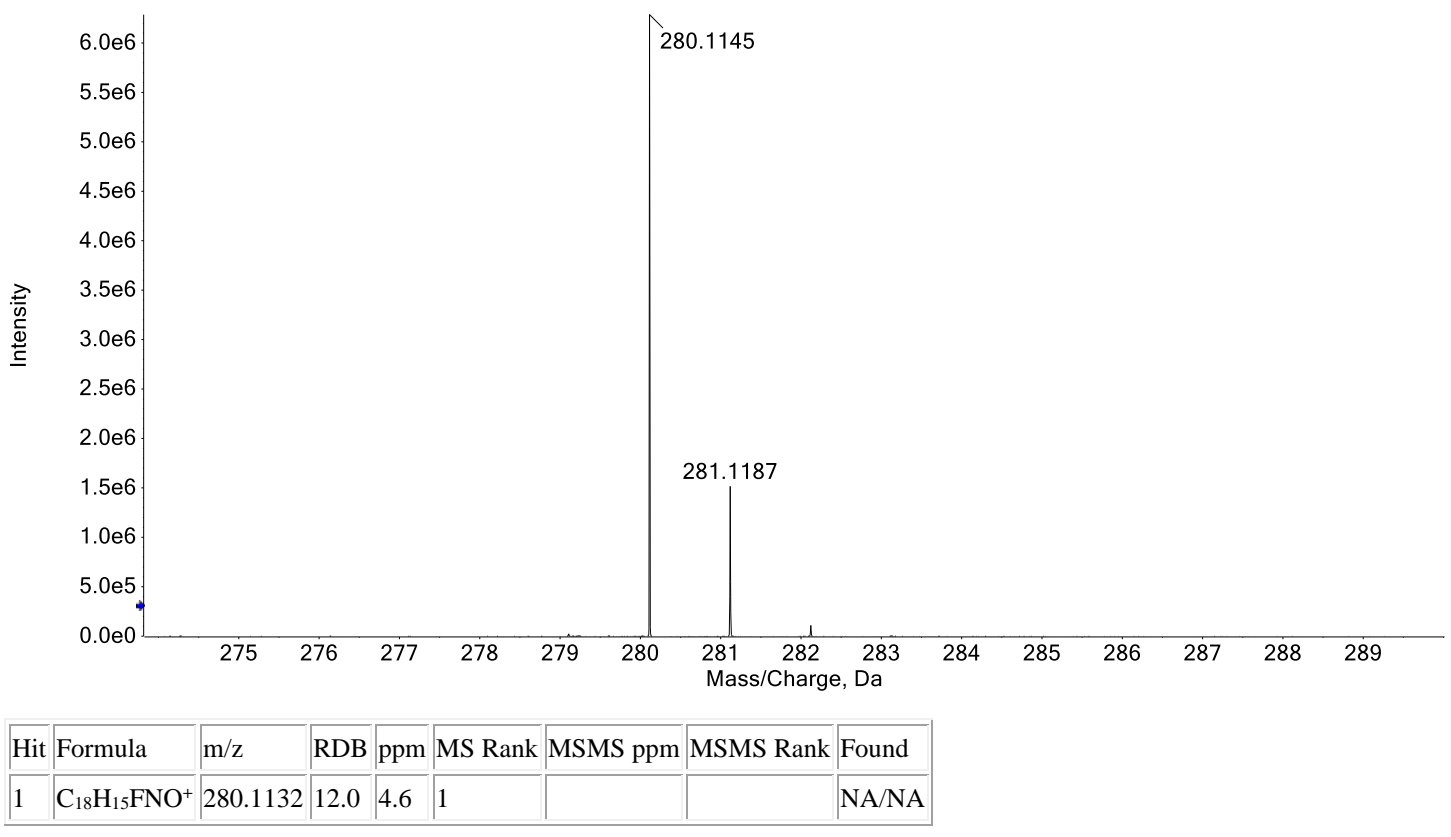

HPLC analysis of $\mathbf{2 i}$

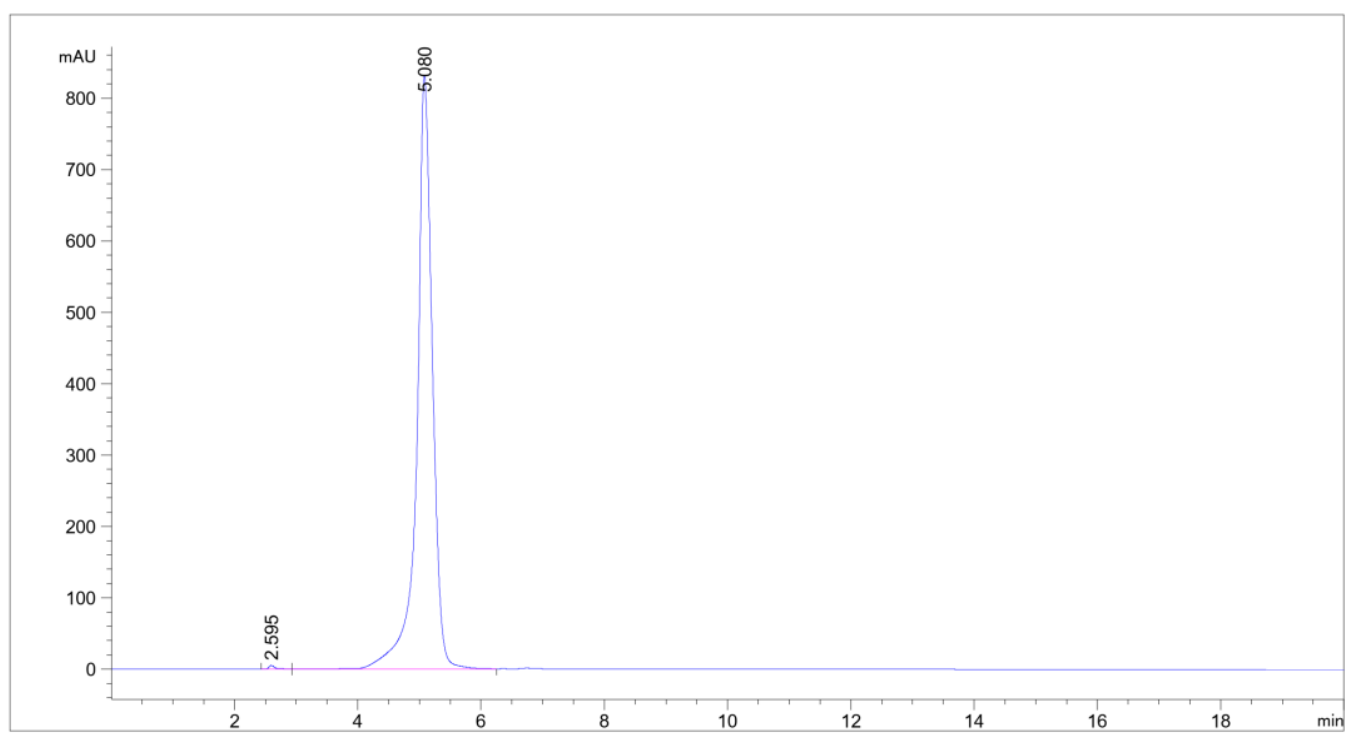

Area Percent Report

$\begin{array}{lcccc}\text { Sorted By } & : & \text { Signal } & \\ \text { Multiplier } & : & 1.0000 & \\ \text { Dilution } & : & 1.0000 & \\ \text { Sample Amount: } & & : \quad 10.00000 \quad \text { [ng/ul] } & \text { (not used in calc.) } \\ \text { Use Multiplier \& Dilution Factor with ISTDs } & \end{array}$

Use Multiplier \& Dilution Factor with ISTDs

Signal 1: VWD1 A, Wavelength=254 nm

\begin{tabular}{|c|c|c|c|c|c|c|}
\hline $\begin{array}{c}\text { Peak } \\
\text { \# }\end{array}$ & $\begin{array}{c}\text { RetTime } \\
\text { [min] }\end{array}$ & Type & $\begin{array}{l}\text { Width } \\
\text { [min] }\end{array}$ & $\begin{array}{c}\text { Area } \\
{\left[\mathrm{mAU}{ }^{\star} \mathrm{s}\right]}\end{array}$ & $\begin{array}{l}\text { Height } \\
\text { [mAU] }\end{array}$ & $\begin{array}{c}\text { Area } \\
\frac{2}{\partial}\end{array}$ \\
\hline & & & 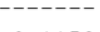 & |--------- & ------ & ------- \\
\hline 1 & 2.595 & BV $R$ & 0.1153 & 40.61032 & 5.36602 & 0.2783 \\
\hline 2 & 5.080 & VB $R$ & 0.2543 & $1.45499 \mathrm{e} 4$ & 830.77362 & 99.7217 \\
\hline Total & $1 \mathrm{~s}:$ & & & $1.45905 \mathrm{e} 4$ & 836.13965 & \\
\hline
\end{tabular}


${ }^{1} \mathrm{H}-\mathrm{NMR}$ spectrum of compound $\mathbf{2} \mathbf{j}$

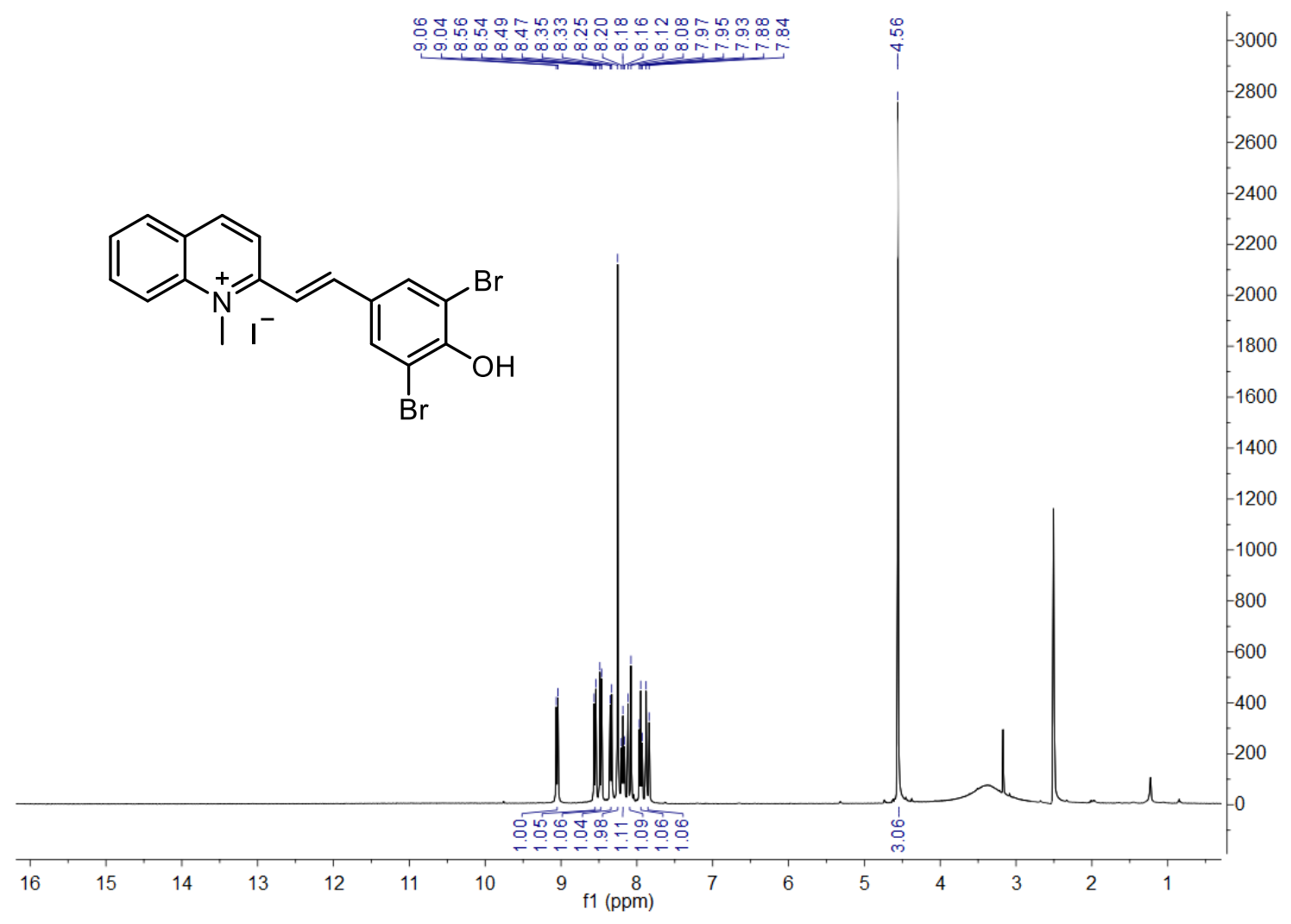

${ }^{13} \mathrm{C}$-NMR spectrum of compound $\mathbf{2} \mathbf{j}$ 


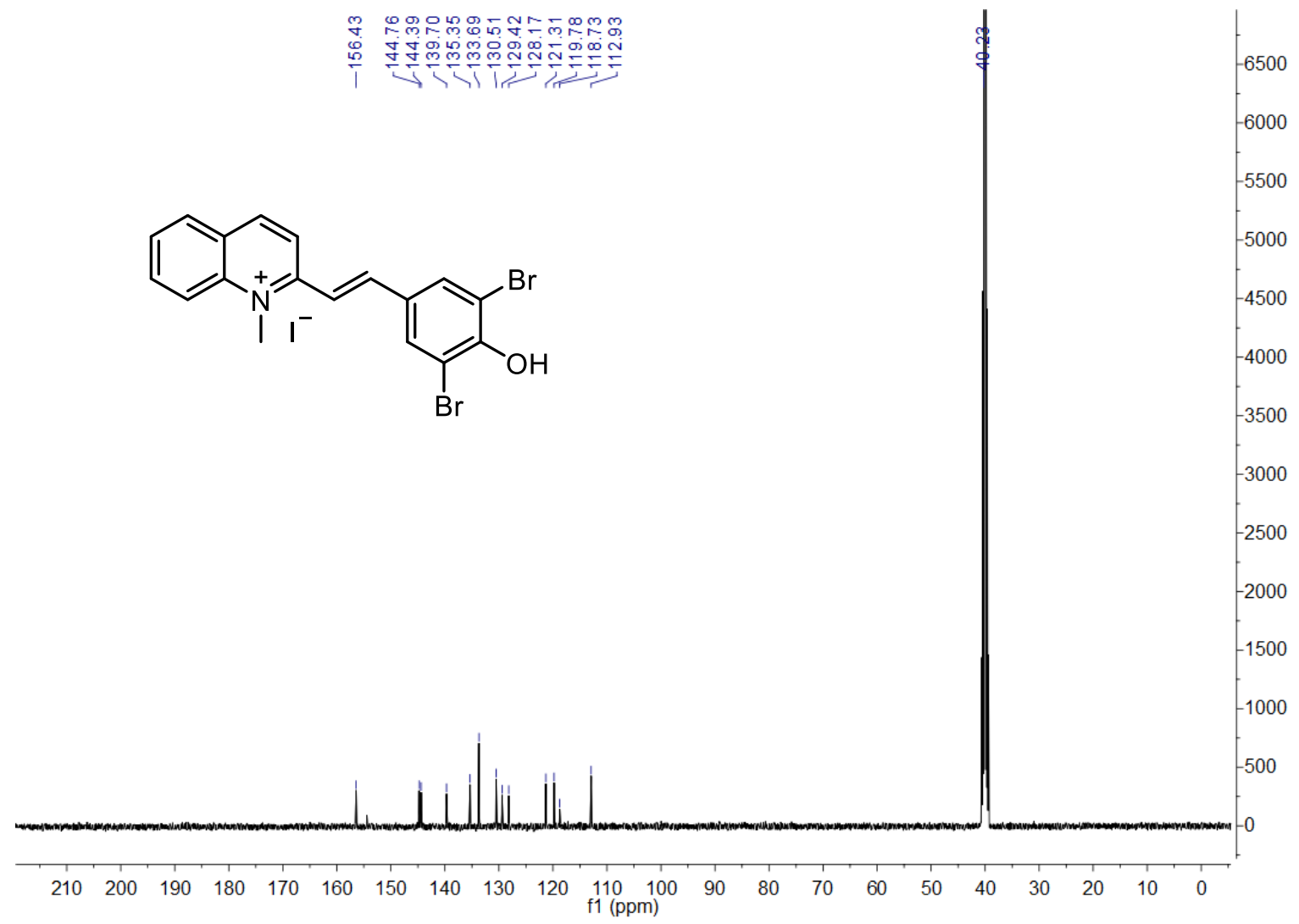

HRMS of compound $\mathbf{2} \mathbf{j}$

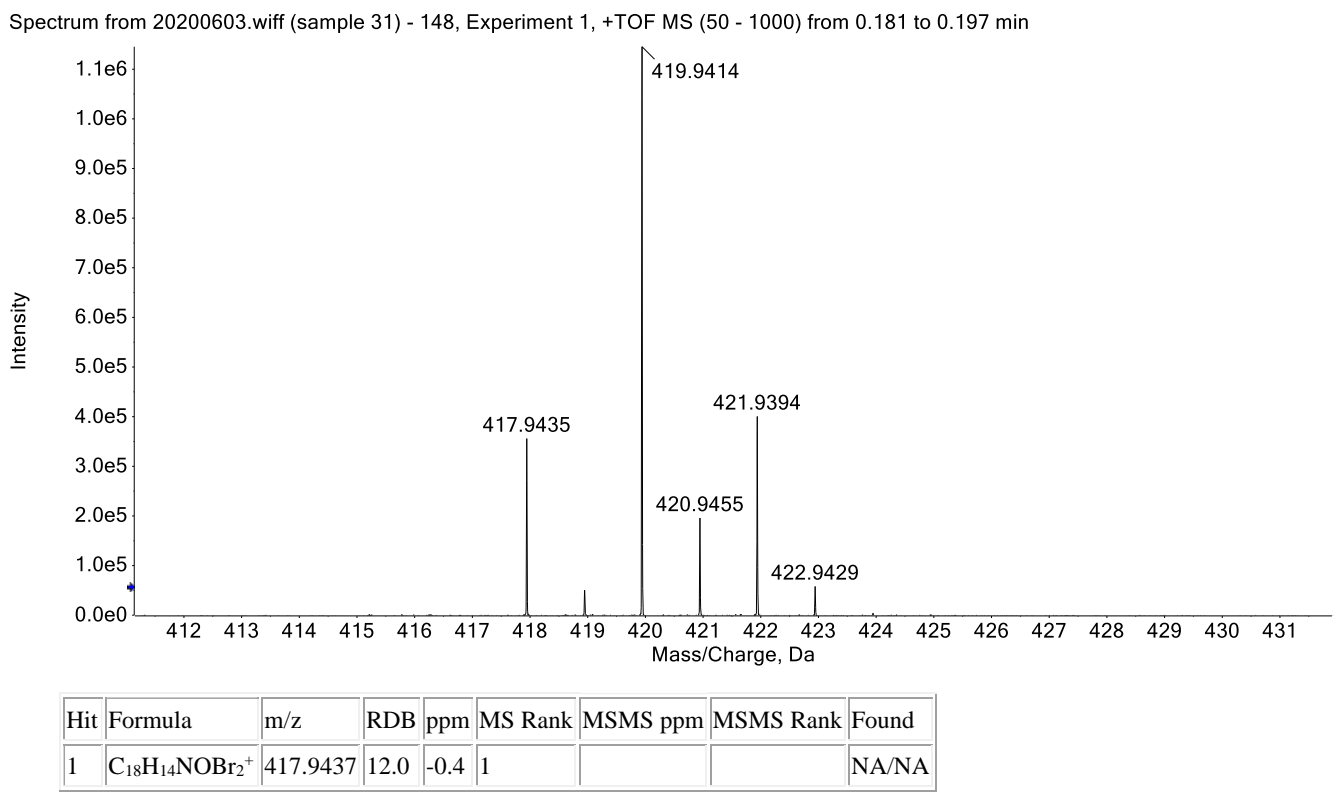


HPLC analysis of $\mathbf{2 j}$

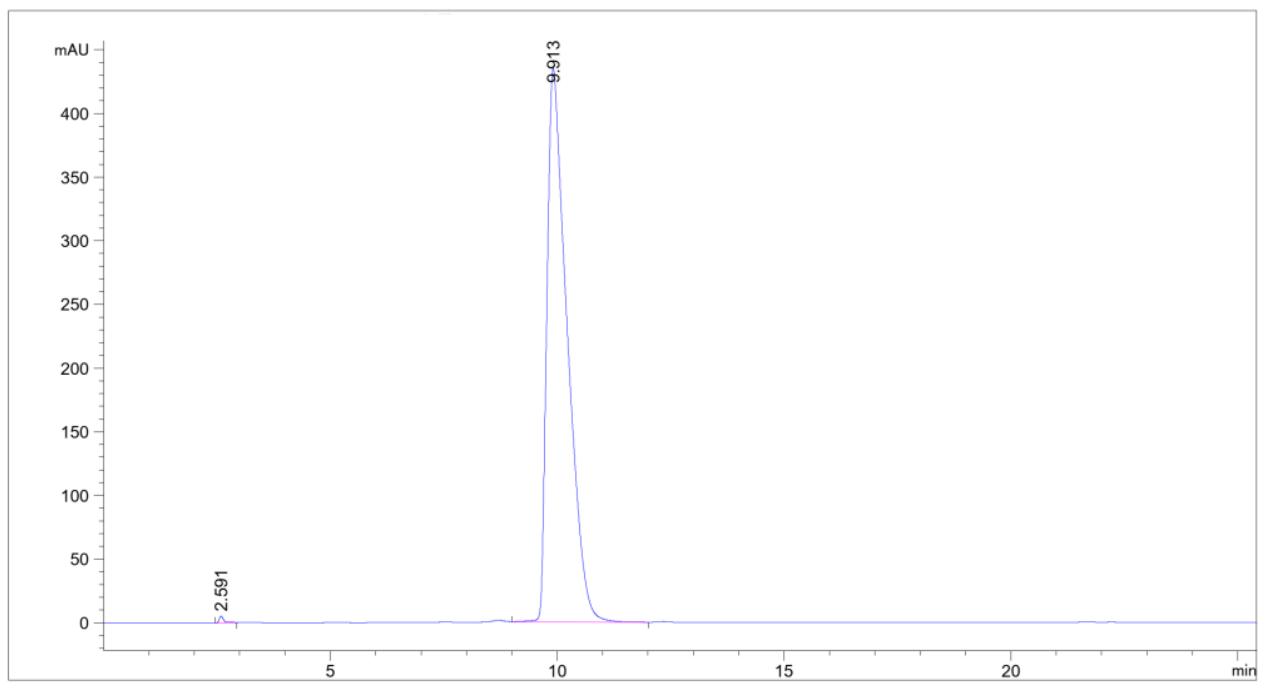

Area Percent Report

$\begin{array}{llll}\text { Sorted By } & : & \text { Signal } & \\ \text { Multiplier } & : & 1.0000 & \\ \text { Dilution } & : & 1.0000 & \\ \text { Sample Amount: } & & : & 10.00000 \quad \text { [ng/ul] }\end{array}$

Use Multiplier \& Dilution Factor with ISTDs

Signal 1: VWD1 A, Wavelength $=254 \mathrm{~nm}$

\begin{tabular}{|c|c|c|c|c|c|c|}
\hline $\begin{array}{c}\text { Peak } \\
\#\end{array}$ & $\begin{array}{c}\text { RetTime } \\
\text { [min] }\end{array}$ & Type & $\begin{array}{l}\text { Width } \\
\text { [min] }\end{array}$ & $\begin{array}{c}\text { Area } \\
{\left[\mathrm{mAU}{ }^{*} \mathrm{~s}\right]}\end{array}$ & $\begin{array}{l}\text { Height } \\
\text { [mAU] }\end{array}$ & $\begin{array}{c}\text { Area } \\
\text { \& }\end{array}$ \\
\hline 1 & $=591$ & BV $R$ & 0 & 39.45232 & 5.10029 & 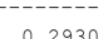 \\
\hline 2 & 9.913 & $\mathrm{BB}$ & 0.4451 & $1.34250 \mathrm{e} 4$ & 434.95102 & 99.7070 \\
\hline
\end{tabular}

Totals : $\quad 1.34645 \mathrm{e} 4 \quad 440.05131$ 


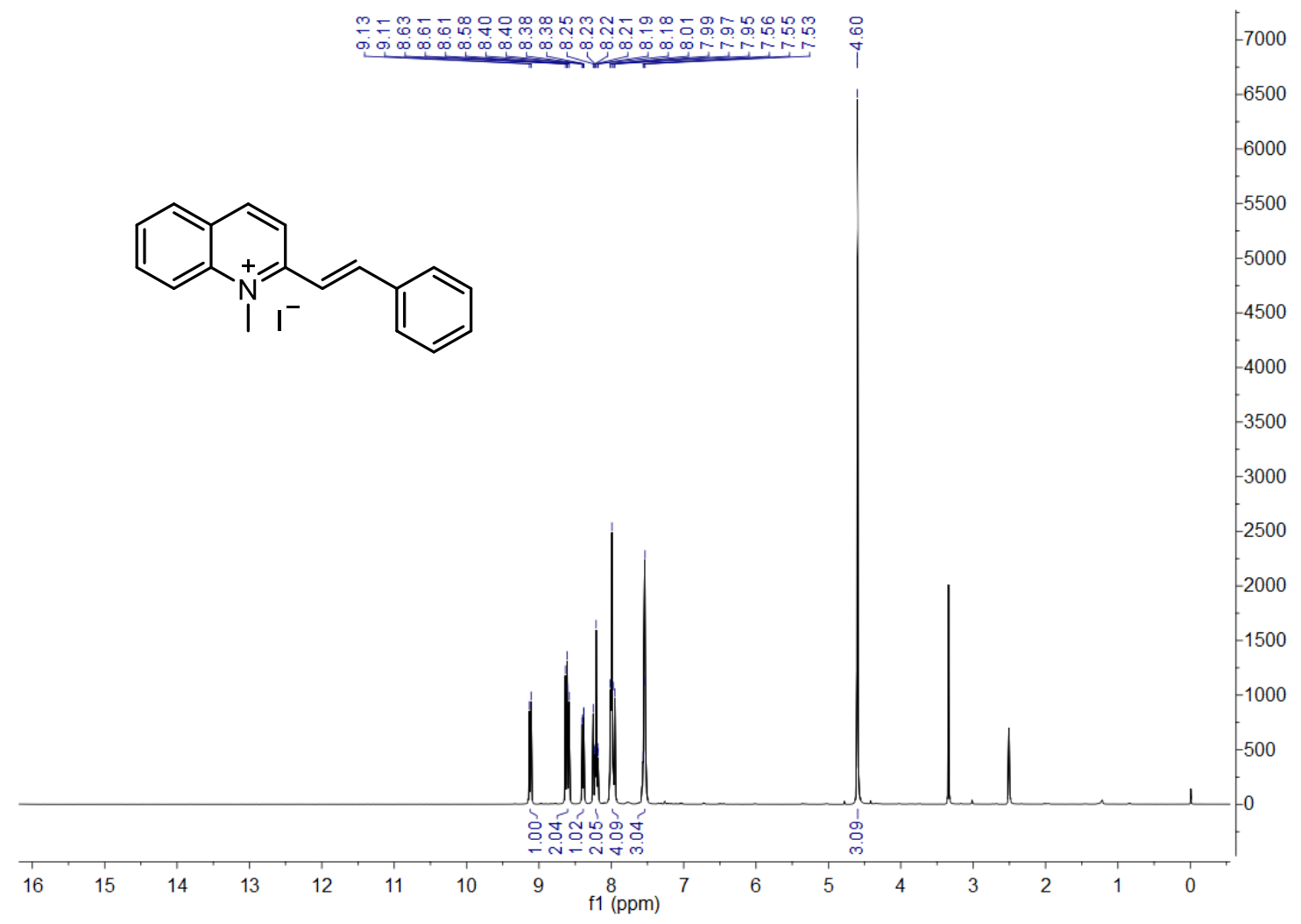

${ }^{13} \mathrm{C}-\mathrm{NMR}$ spectrum of compound $\mathbf{2 k}$

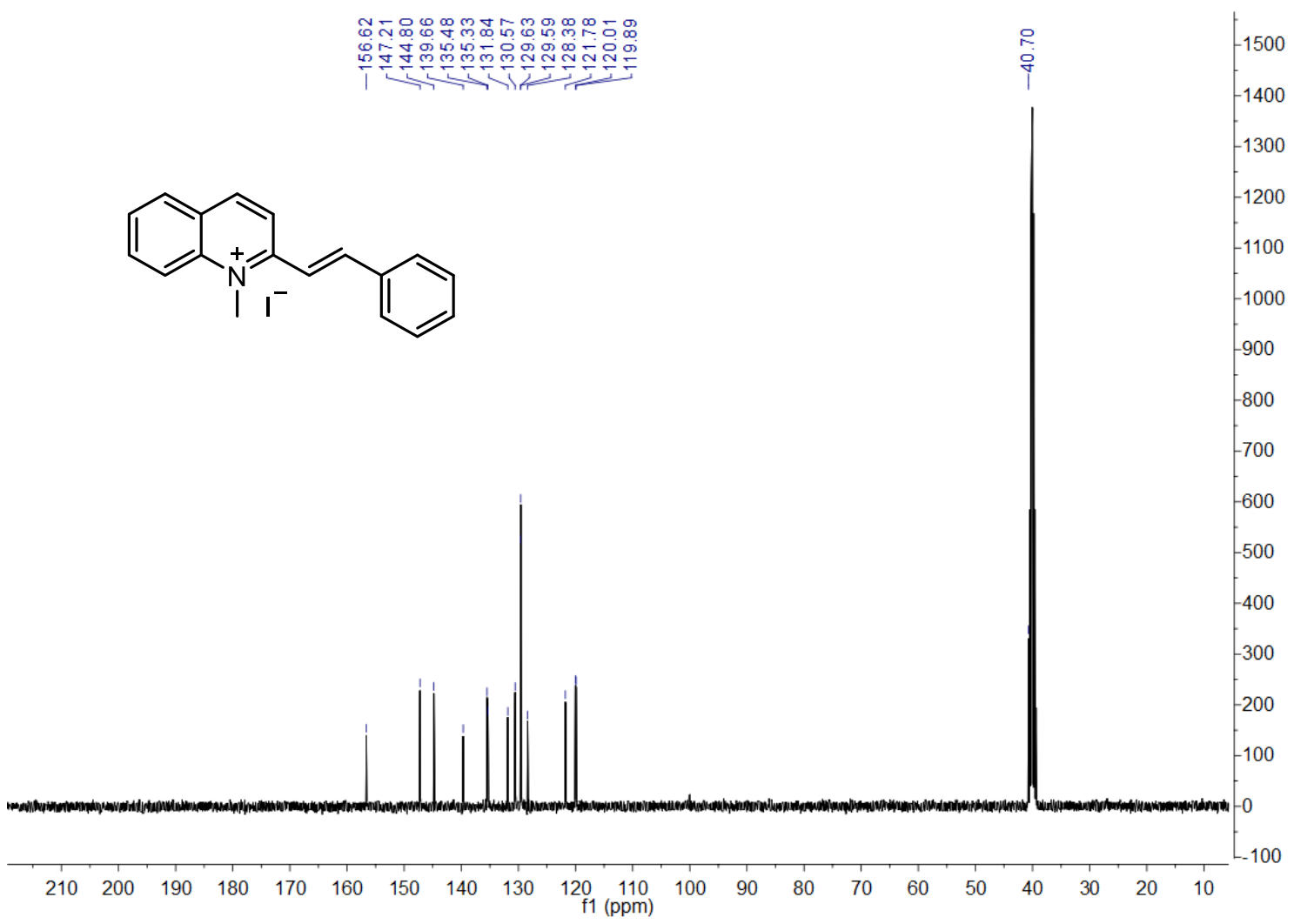

HRMS of compound $\mathbf{2 k}$

S33 
Spectrum from 20200603. wiff (sample 32) - 166, Experiment 1, +TOF MS (50 - 1000) from 0.180 to $0.196 \mathrm{~min}$

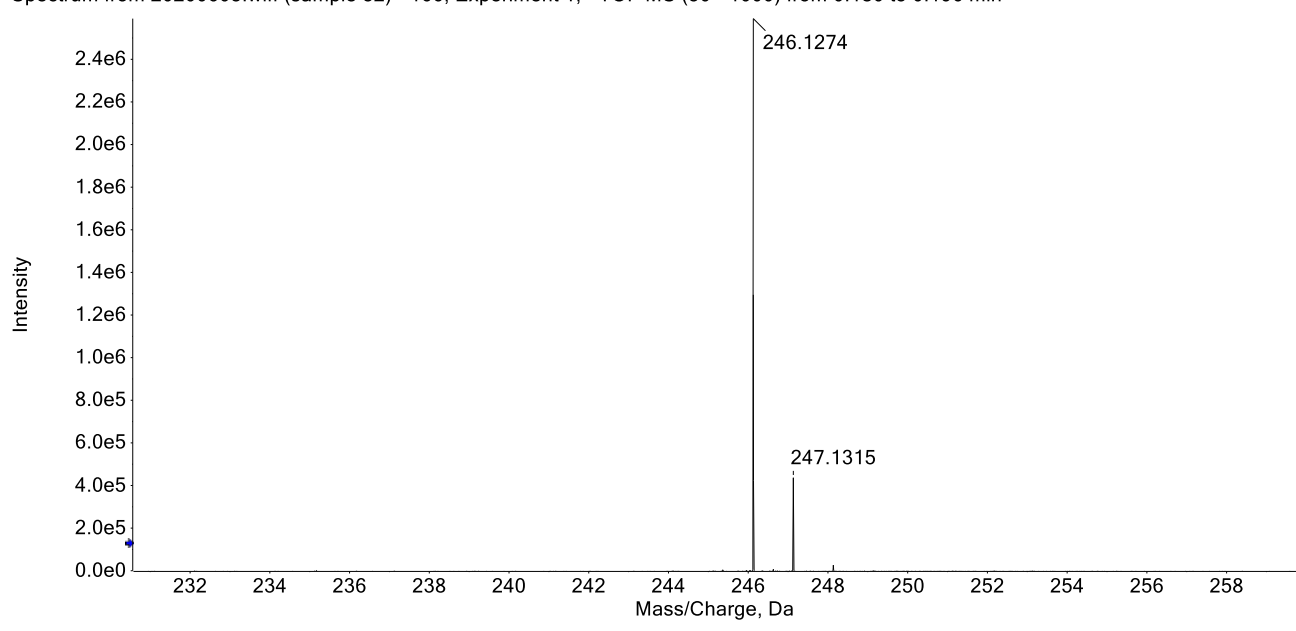

$\begin{array}{llllll}\text { Hit Formula } & \text { m/z } & \text { RDB } & \text { ppm MS Rank MSMS ppm MSMS Rank Found }\end{array}$

\begin{tabular}{|l|l|l|l|l|l|l|l|}
\hline 1 & $\mathrm{C}_{18} \mathrm{H}_{16} \mathrm{~N}^{+}$ & 246.1277 & 12.0 & -1.3 & 1 & & NA/NA \\
\hline
\end{tabular}

HPLC analysis of $\mathbf{2 k}$

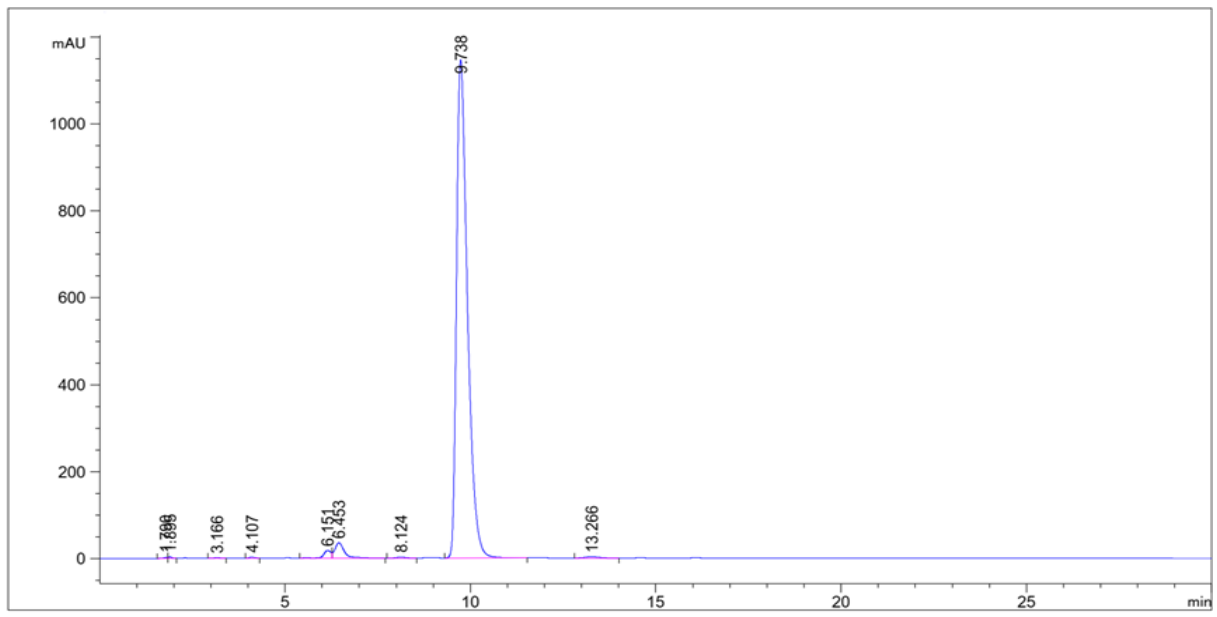

Area Percent Report

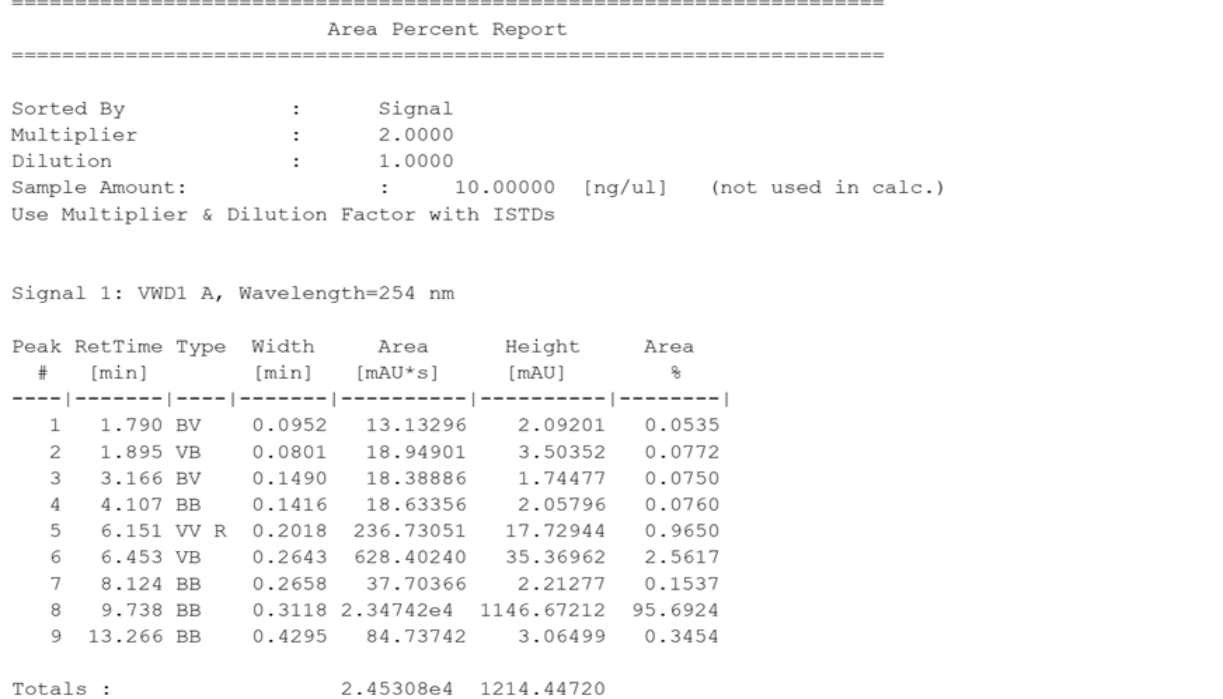


${ }^{1} \mathrm{H}-\mathrm{NMR}$ spectrum of compound $\mathbf{2 l}$

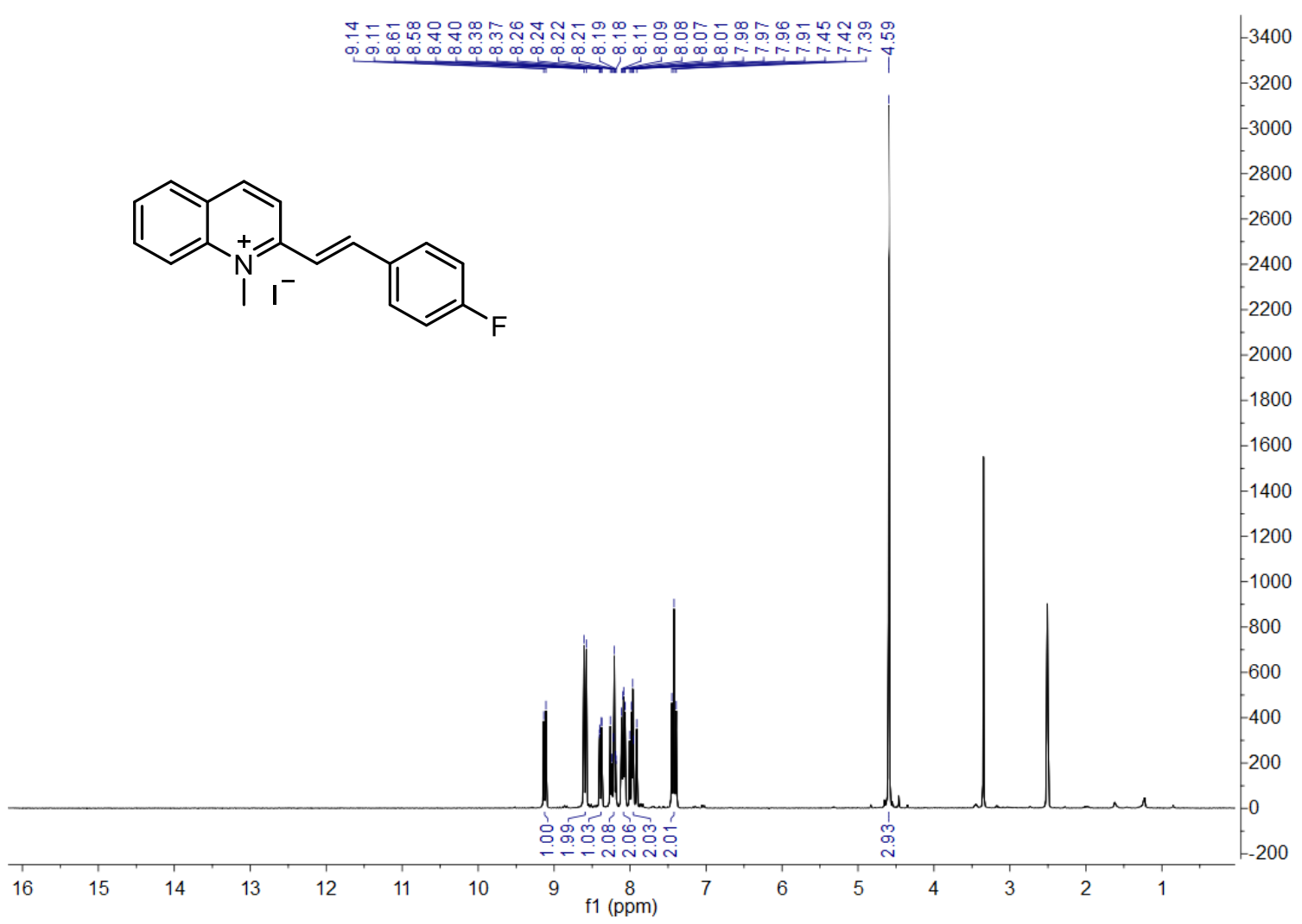

${ }^{13} \mathrm{C}-\mathrm{NMR}$ spectrum of compound $\mathbf{2 I}$

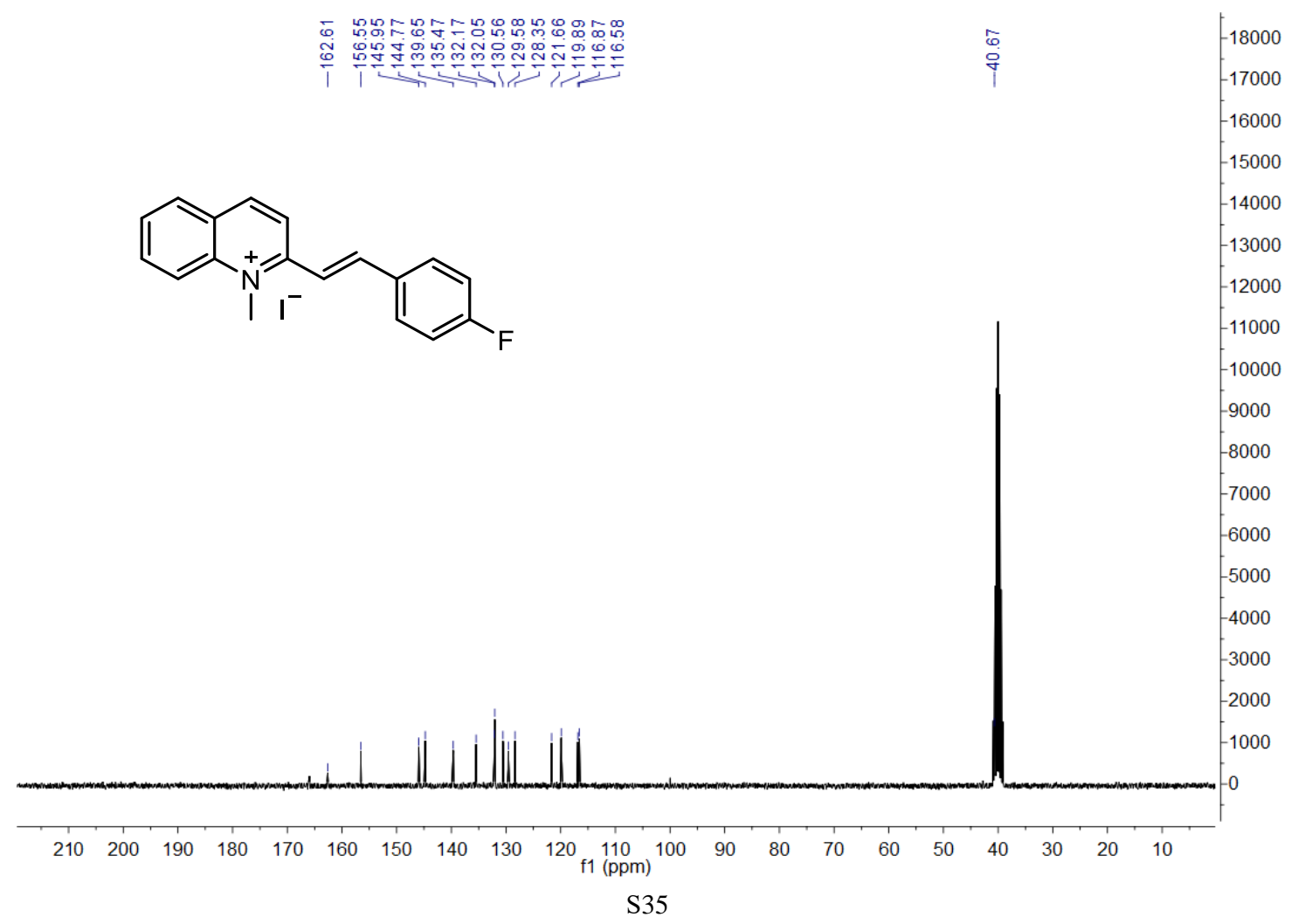




\section{HRMS of compound 2l}

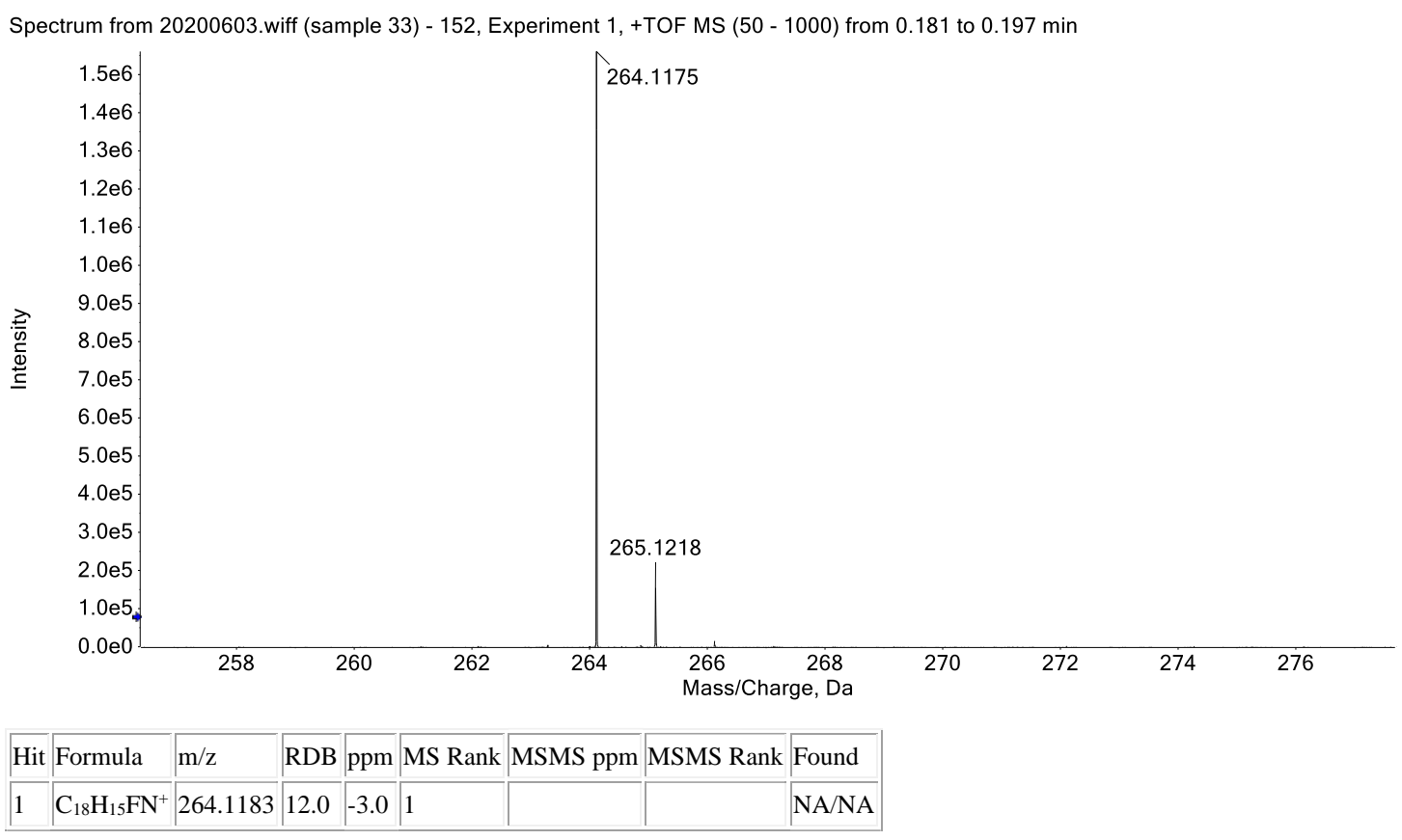

HPLC analysis of $\mathbf{2 l}$ 


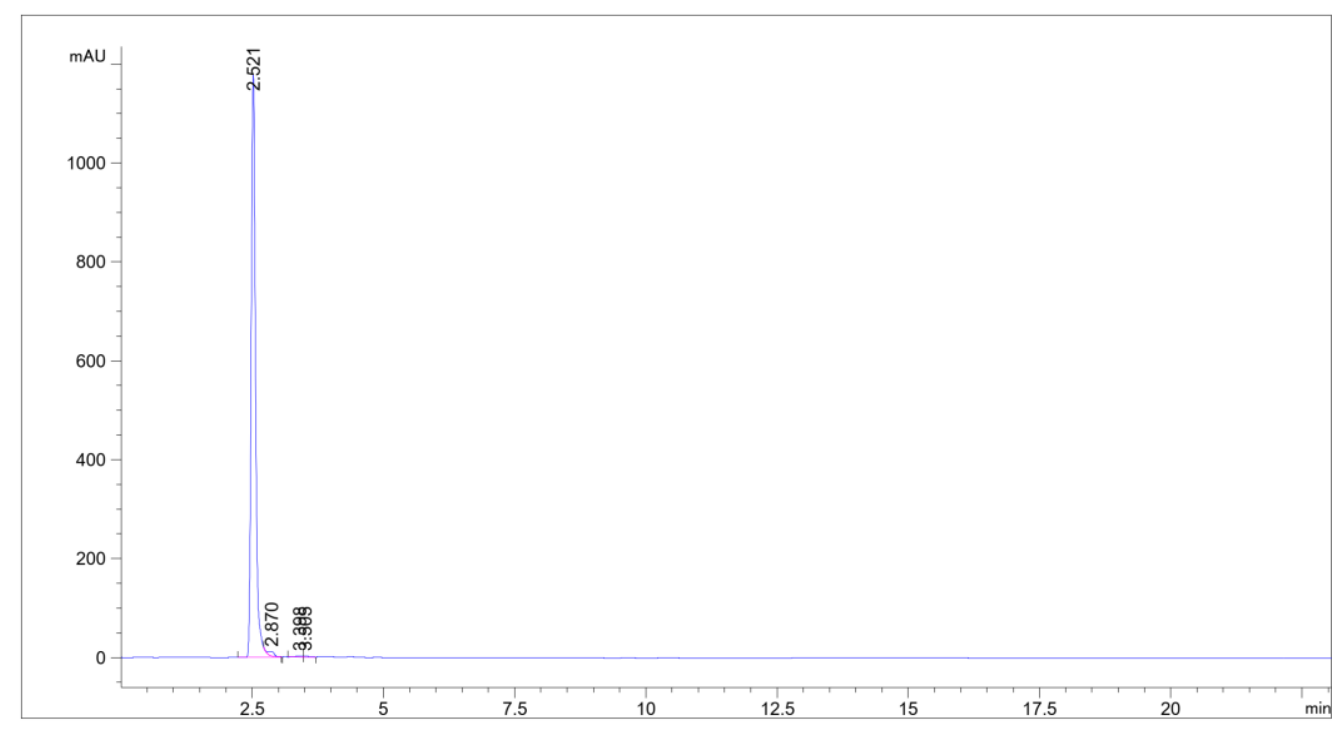

Area Percent Report

$\begin{array}{lcccc}\text { Sorted By } & : & \text { Signal } & \\ \text { Multiplier } & : & 1.0000 & \\ \text { Dilution } & : & 1.0000 & \\ \text { Sample Amount: } & & : \quad 10.00000 \quad \text { [ng/ul] } & \text { (not used in calc.) } \\ \text { Use Multiplier \& Dilution Factor with ISTDs } & \end{array}$

Use Multiplier \& Dilution Factor with ISTDs

Signal 1: VWD1 A, Wavelength=250 nm

\begin{tabular}{|c|c|c|c|c|c|c|}
\hline $\begin{array}{c}\text { Peak } \\
\quad \#\end{array}$ & $\begin{array}{c}\text { RetTime } \\
\text { [min] }\end{array}$ & Type & $\begin{array}{l}\text { Width } \\
\text { [min] }\end{array}$ & $\begin{array}{c}\text { Area } \\
{\left[\mathrm{mAU}{ }^{*} \mathrm{~s}\right]}\end{array}$ & $\begin{array}{l}\text { Height } \\
\text { [mAU] }\end{array}$ & $\begin{array}{c}\text { Area } \\
\frac{\circ}{0}\end{array}$ \\
\hline 1 & 2.521 & BV $R$ & 0.0885 & 6815.10107 & 1177.23804 & 98.4651 \\
\hline 2 & 2.870 & VB E & 0.1170 & 72.12989 & 9.04586 & 1.0421 \\
\hline 3 & 3.398 & BV & 0.1305 & 20.62197 & 2.13827 & 0.2979 \\
\hline 4 & 3.505 & VB & 0.1084 & 13.48575 & 1.82071 & 0.1948 \\
\hline Total & $1 s:$ & & & 6921.33868 & 1190.24288 & \\
\hline
\end{tabular}

${ }^{1} \mathrm{H}-\mathrm{NMR}$ spectrum of compound $\mathbf{2 m}$ 

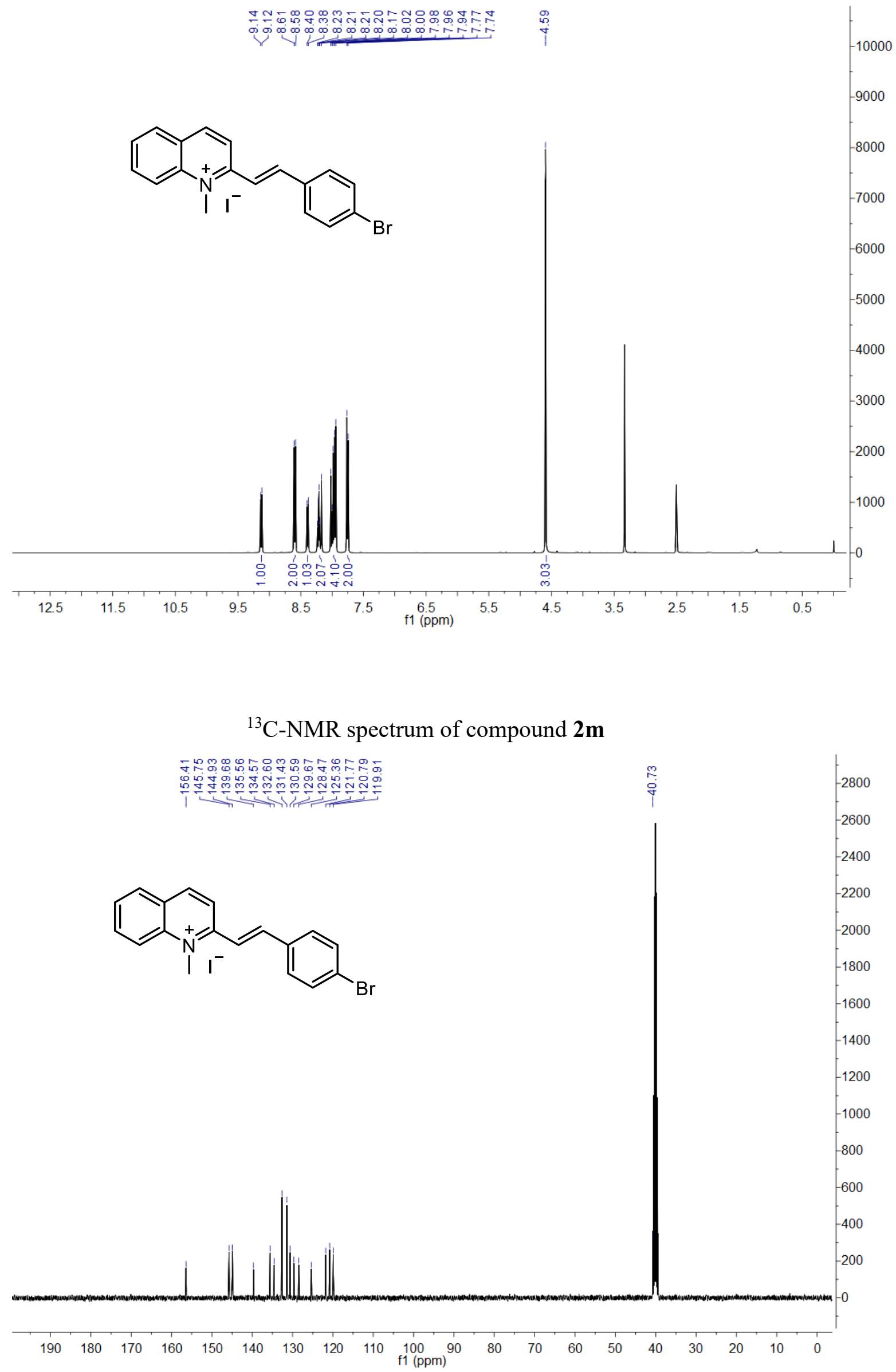
HRMS of compound $\mathbf{2 m}$

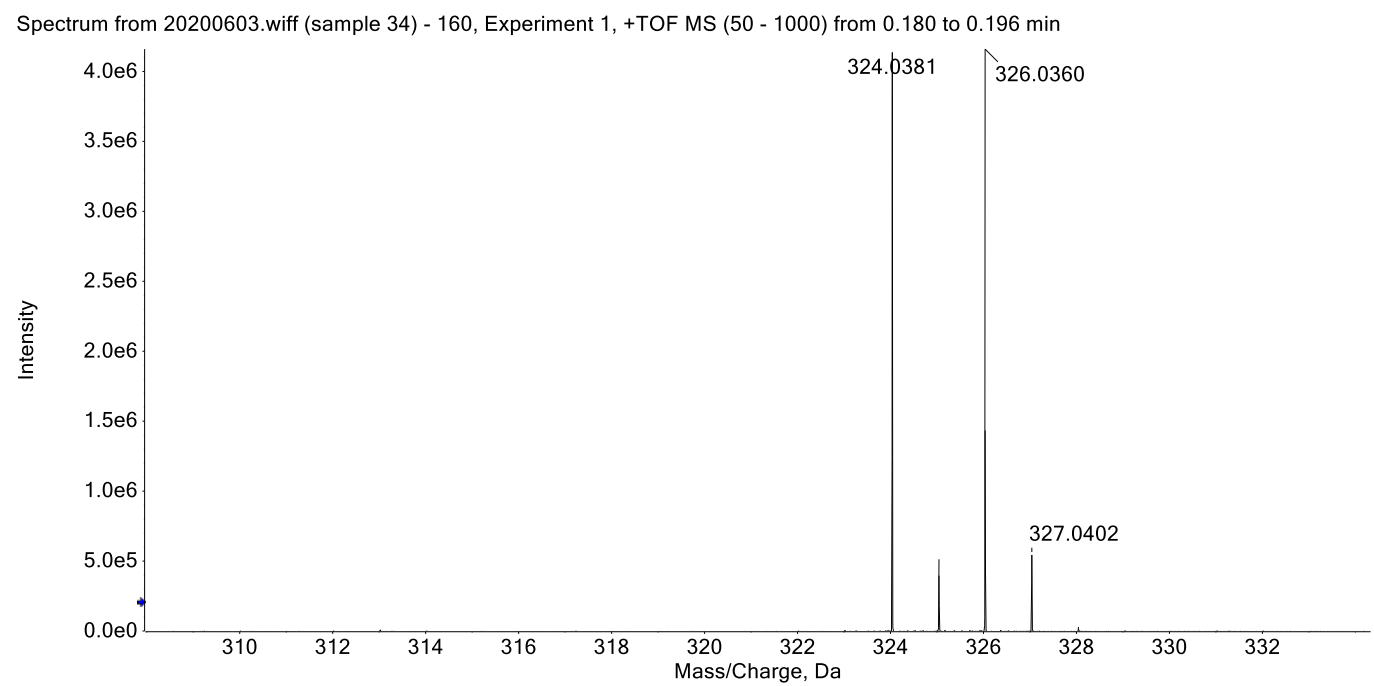

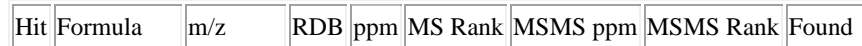

\begin{tabular}{|l|l|l|l|l|l|l|}
\hline 1 & $\mathrm{C}_{18} \mathrm{H}_{15} \mathrm{BrN}^{+}$ & 324.0382 & 12.0 & -0.4 & 1 & \\
\hline
\end{tabular}

\section{HPLC analysis of $\mathbf{2} \mathbf{m}$}

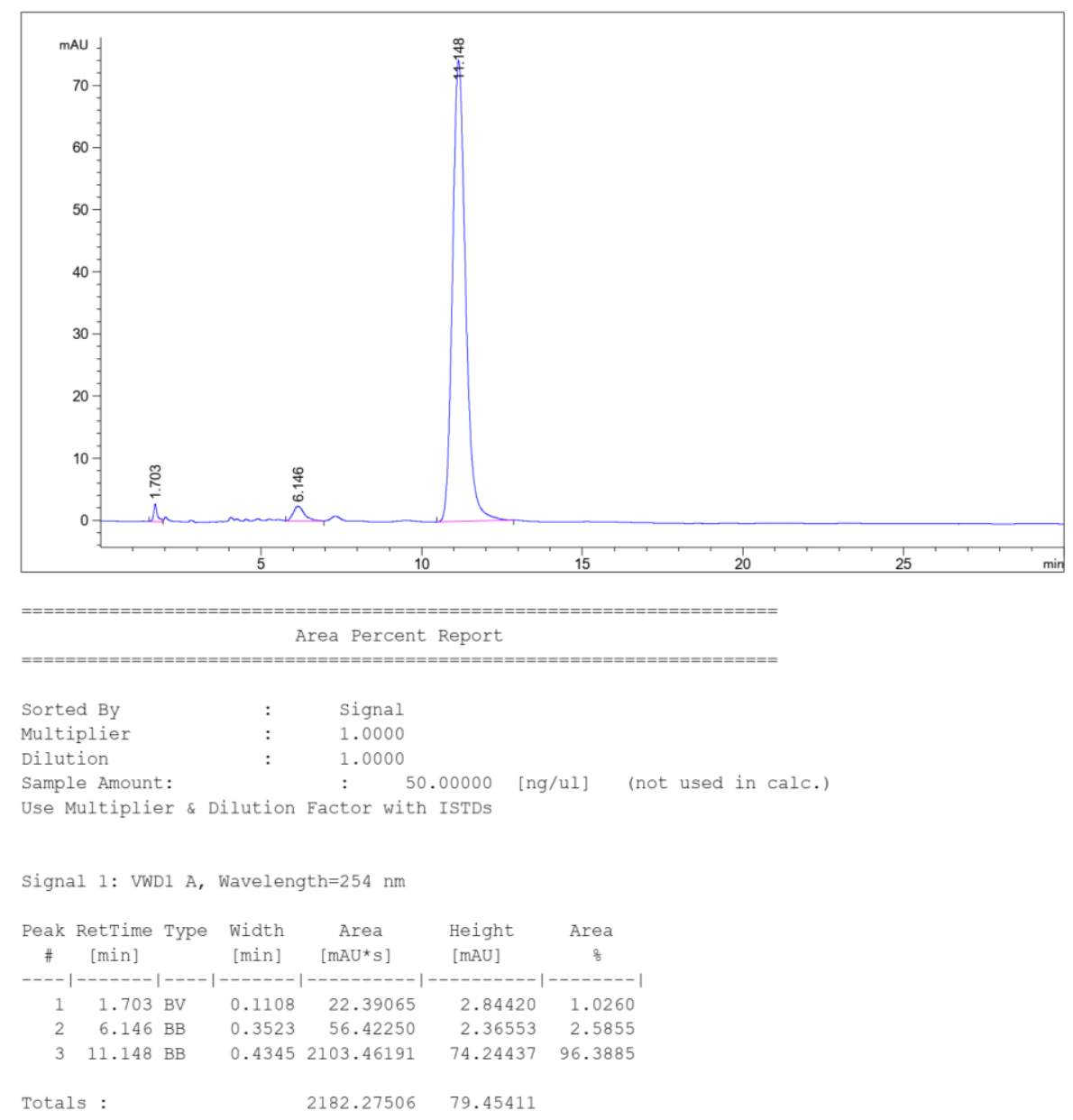




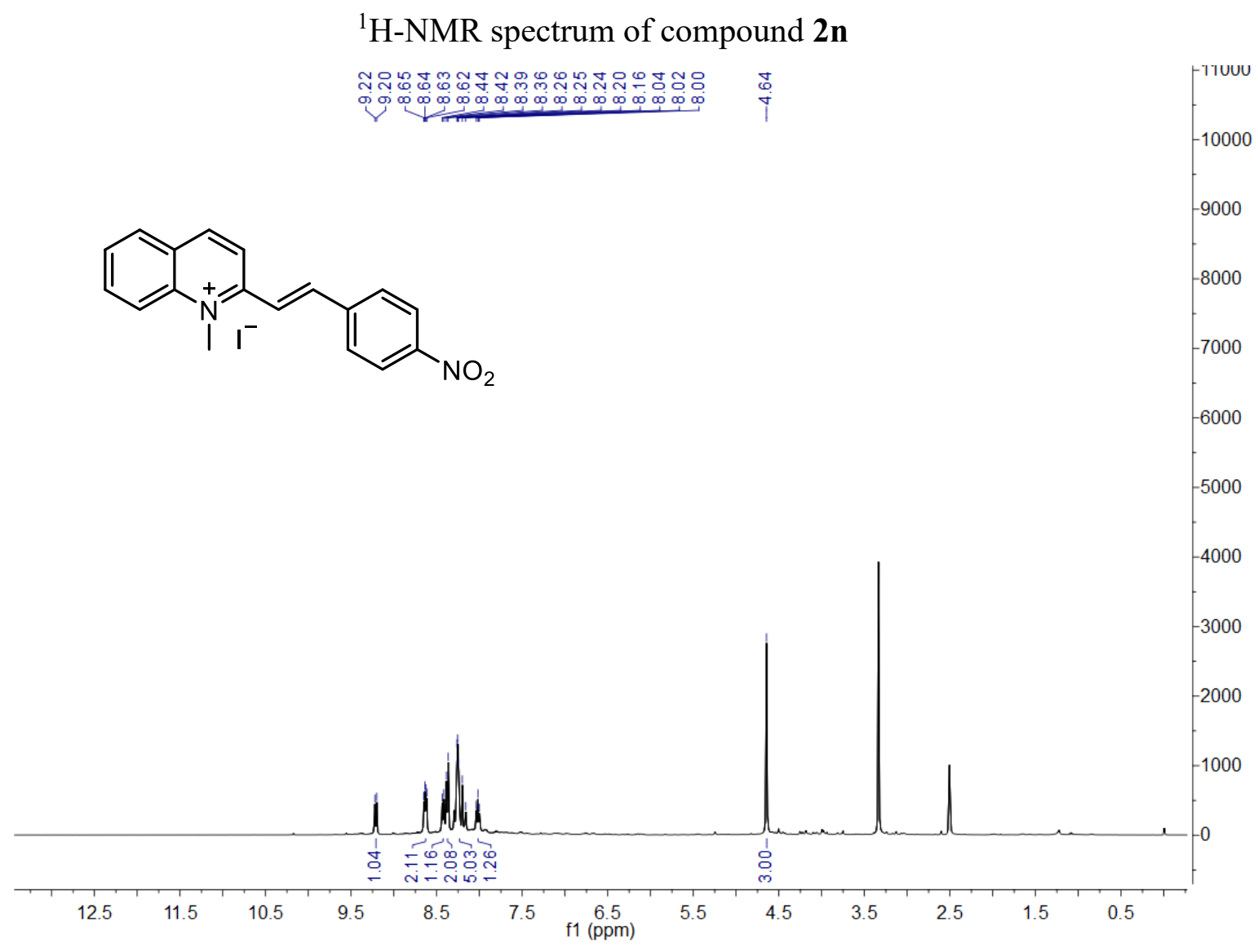

${ }^{13} \mathrm{C}-\mathrm{NMR}$ spectrum of compound $\mathbf{2 n}$ 


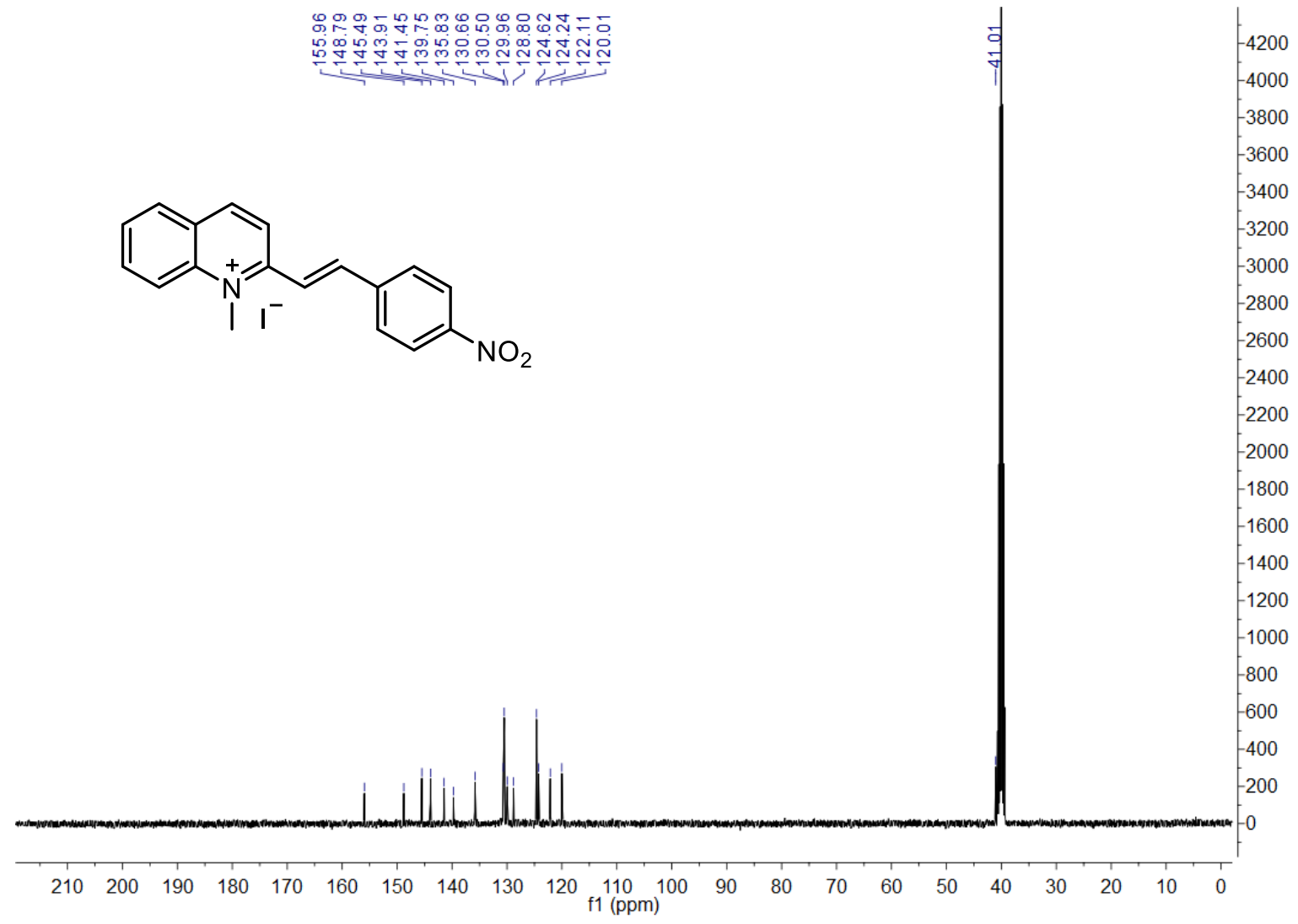

HRMS of compound 2n

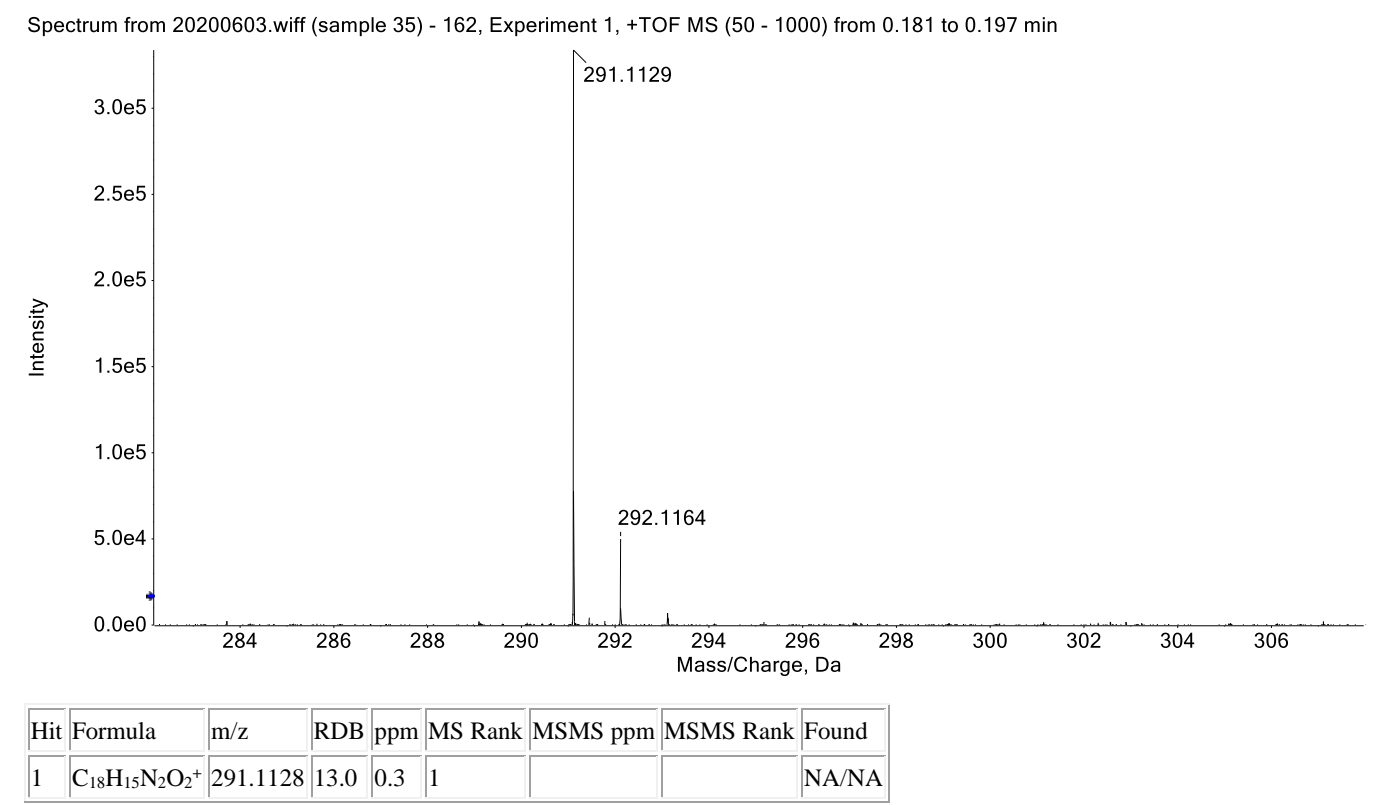

HPLC analysis of $\mathbf{2 n}$ 


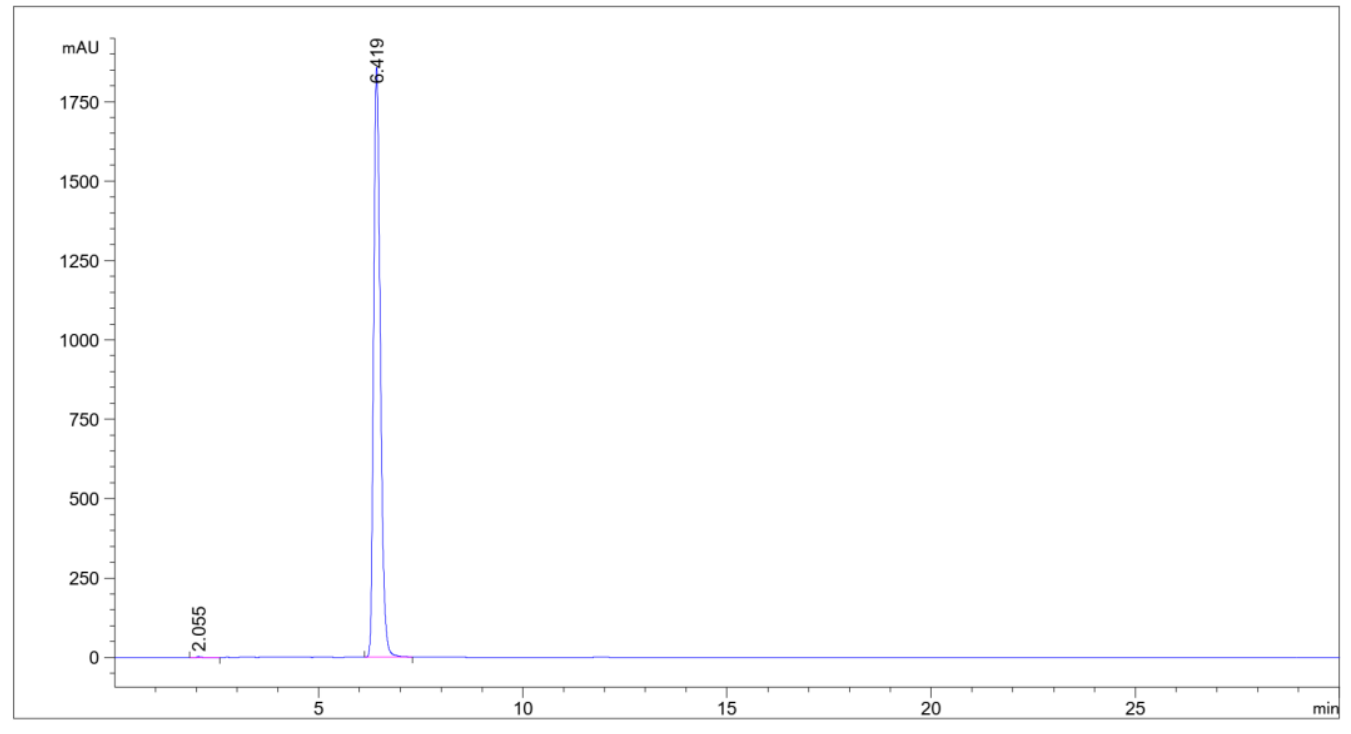

Area Percent Report

$\begin{array}{lccc}\text { Sorted By } & : & \text { Signal } & \\ \text { Multiplier } & : & 1.0000 \\ \text { Dilution } & : & 1.0000 \\ \text { Sample Amount: } & & : \quad 30.00000 \quad \text { [ng/ul] } \quad \text { (not used in calc.) } \\ \text { Use Multiplier \& Dilution Factor with ISTDs }\end{array}$

Signal 1: VWD1 A, Wavelength $=254 \mathrm{~nm}$

\begin{tabular}{|c|c|c|c|c|c|c|}
\hline $\begin{array}{c}\text { Peak } \\
\#\end{array}$ & $\begin{array}{c}\text { RetTime } \\
\text { [min] }\end{array}$ & Type & $\begin{array}{c}\text { Width } \\
\text { [min] }\end{array}$ & $\begin{array}{c}\text { Area } \\
{\left[\mathrm{mAU}{ }^{*} \mathrm{~S}\right]}\end{array}$ & $\begin{array}{l}\text { Height } \\
\text { [mAU] }\end{array}$ & $\begin{array}{c}\text { Area } \\
\frac{8}{8}\end{array}$ \\
\hline \multicolumn{7}{|c|}{----|-------| } \\
\hline 1 & 2.055 & $\mathrm{BB}$ & 0.0786 & 12.50941 & 2.29588 & 0.0590 \\
\hline 2 & 6.419 & $\mathrm{BB}$ & 0.1772 & $2.12062 \mathrm{e} 4$ & 1856.76416 & 99.9410 \\
\hline Total & : & & & $2.12187 e 4$ & 1859.06004 & \\
\hline
\end{tabular}

${ }^{1} \mathrm{H}-\mathrm{NMR}$ spectrum of compound $\mathbf{3 a}$ 

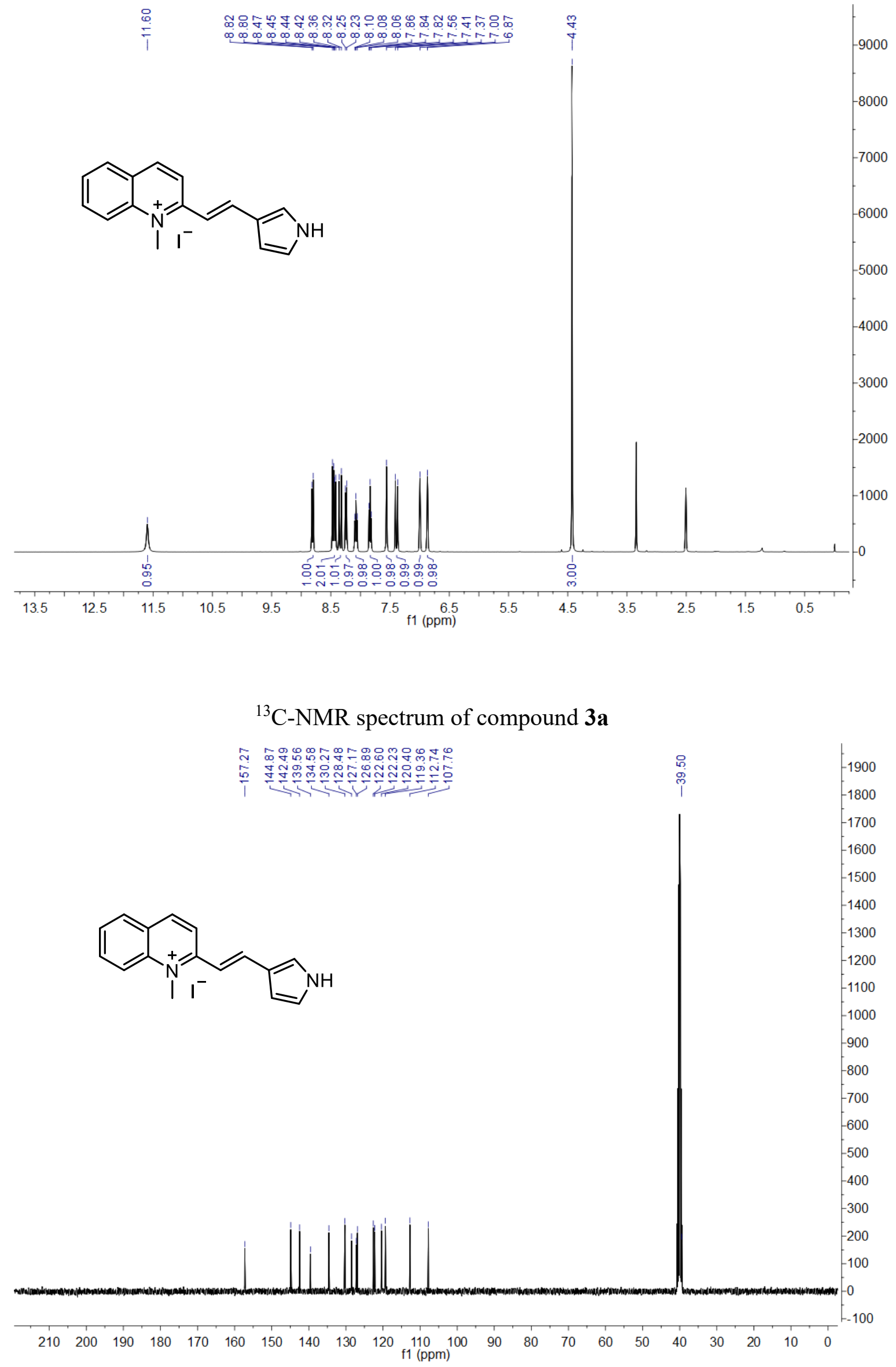
HRMS of compound $\mathbf{3 a}$

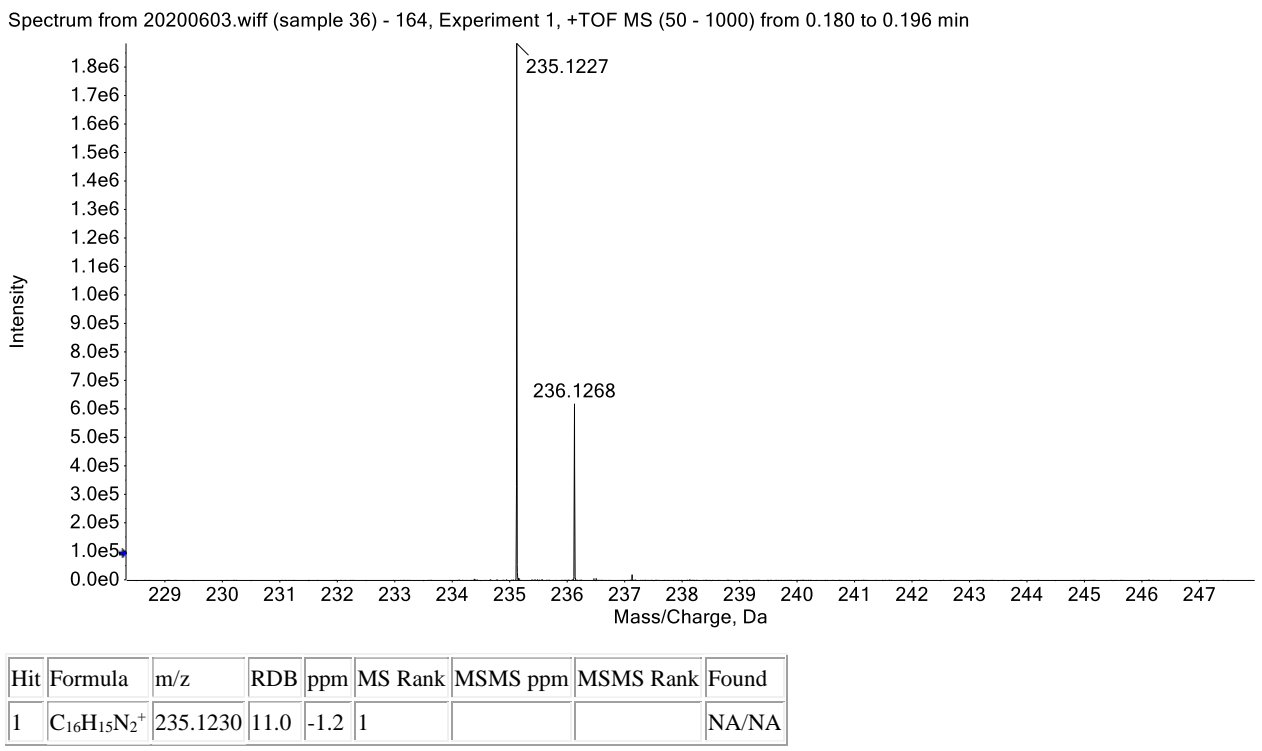

HPLC analysis of $\mathbf{3 a}$ 


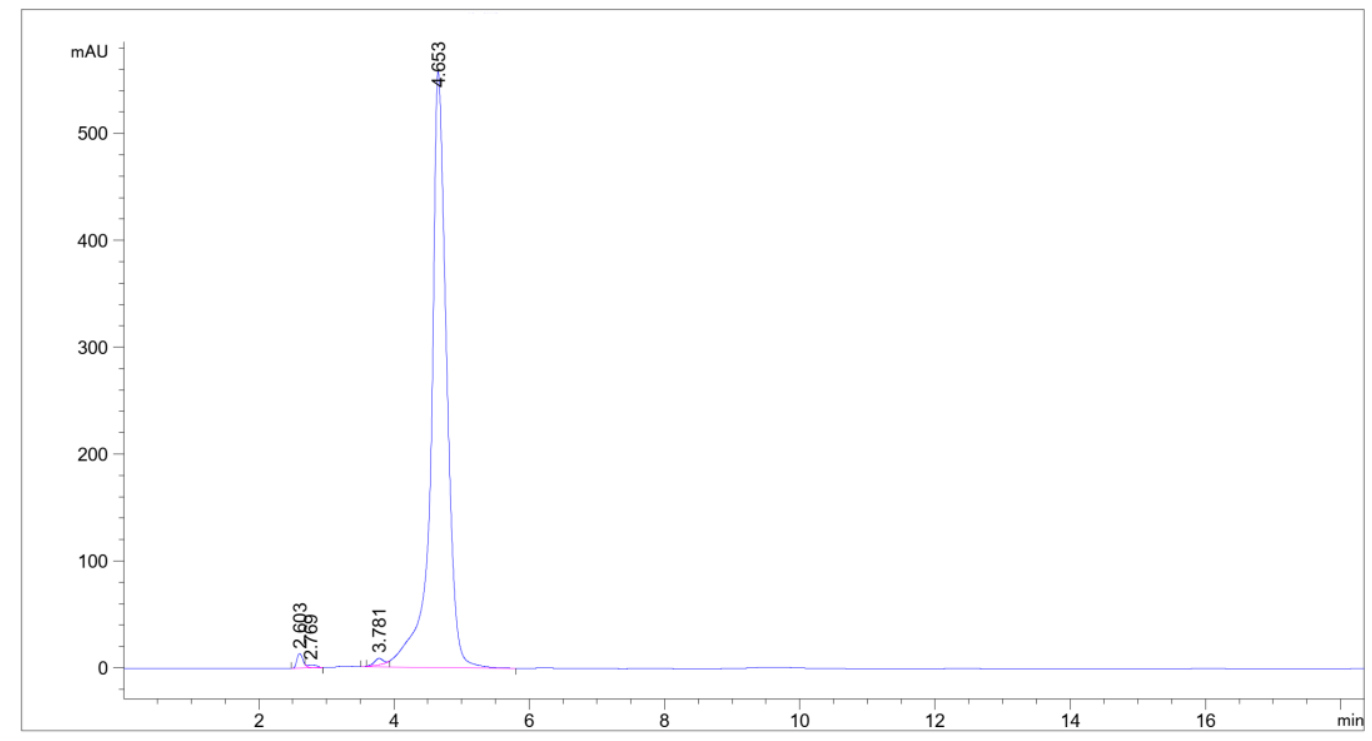

Area Percent Report

$\begin{array}{lccc}\text { Sorted By } & : & \text { Signal } & \\ \text { Multiplier } & : & 1.0000 \\ \text { Dilution } & : & 1.0000 \\ \text { Sample Amount: } & & : \quad 10.00000 \quad \text { [ng/ul] } \quad \text { (not used in calc.) } \\ \text { Use Multiplier \& Dilution Factor with ISTDs }\end{array}$

Signal 1: VWD1 A, Wavelength $=254 \mathrm{~nm}$

\begin{tabular}{|c|c|c|c|c|c|c|}
\hline $\begin{array}{c}\text { Peak } \\
\#\end{array}$ & $\begin{array}{c}\text { RetTime } \\
\text { [min] }\end{array}$ & Type & $\begin{array}{l}\text { Width } \\
\text { [min] }\end{array}$ & $\begin{array}{c}\text { Area } \\
{\left[\mathrm{mAU}{ }^{\star} \mathrm{S}\right]}\end{array}$ & $\begin{array}{l}\text { Height } \\
\text { [mAU] }\end{array}$ & $\begin{array}{c}\text { Area } \\
\frac{2}{0}\end{array}$ \\
\hline- & & & -- & 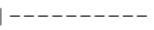 & 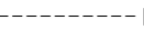 & ------ \\
\hline 1 & 2.603 & $B$ BV $R$ & 0.1014 & 88.48872 & 13.68911 & 0.9675 \\
\hline 2 & 2.769 & 9 VB E & 0.1475 & 21.65497 & 2.39382 & 0.2368 \\
\hline 3 & 3.781 & BV E & 0.1343 & 53.15173 & 6.06109 & 0.5811 \\
\hline 4 & 4.653 & $B$ VB $R$ & 0.2319 & 8983.17969 & 557.76917 & 98.2147 \\
\hline tal & & & & 9146.47511 & 579.91319 & \\
\hline
\end{tabular}

${ }^{1} \mathrm{H}-\mathrm{NMR}$ spectrum of compound $\mathbf{3 b}$ 


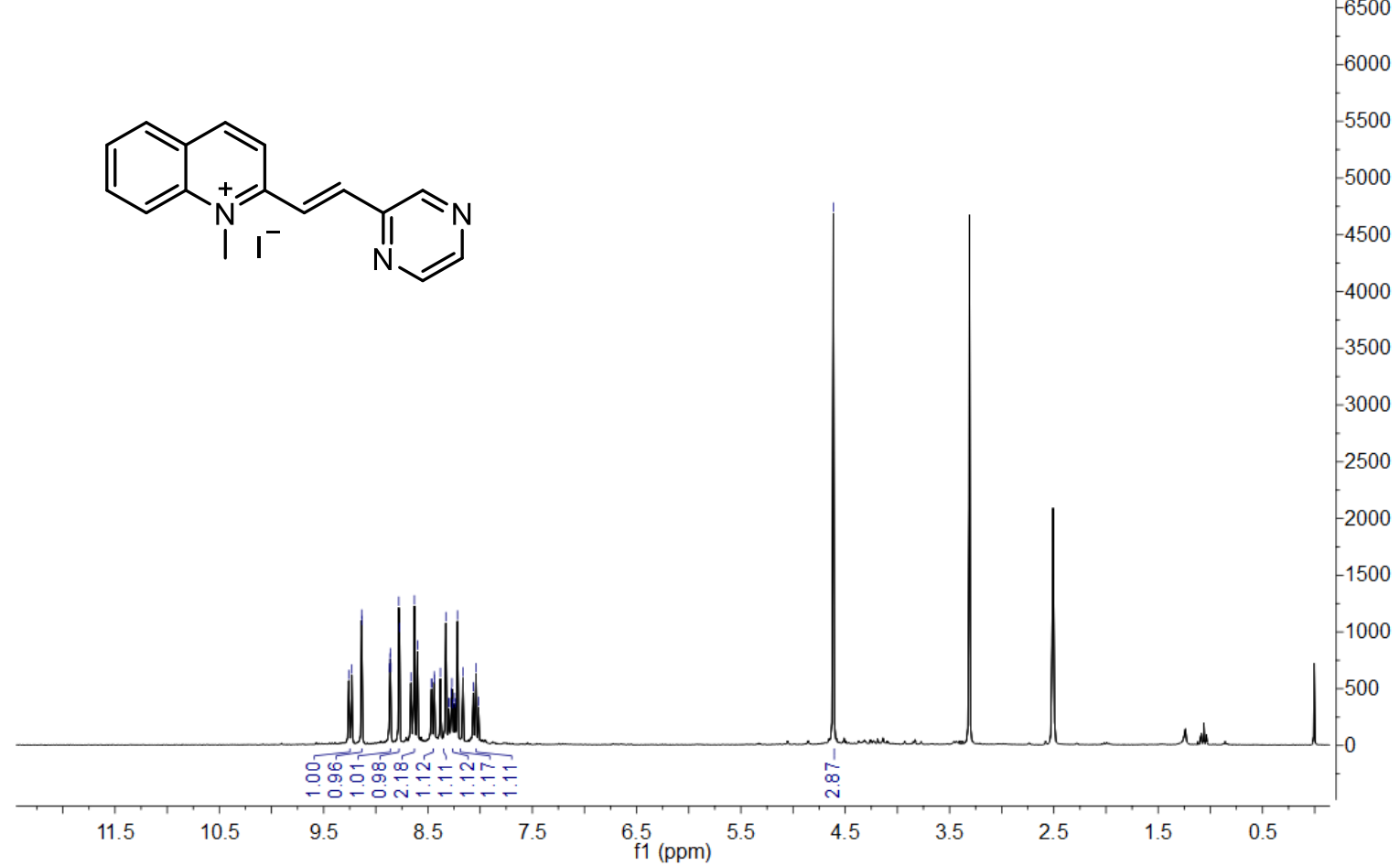

${ }^{13} \mathrm{C}-\mathrm{NMR}$ spectrum of compound $\mathbf{3 b}$

대윰ำ

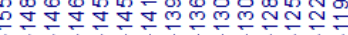
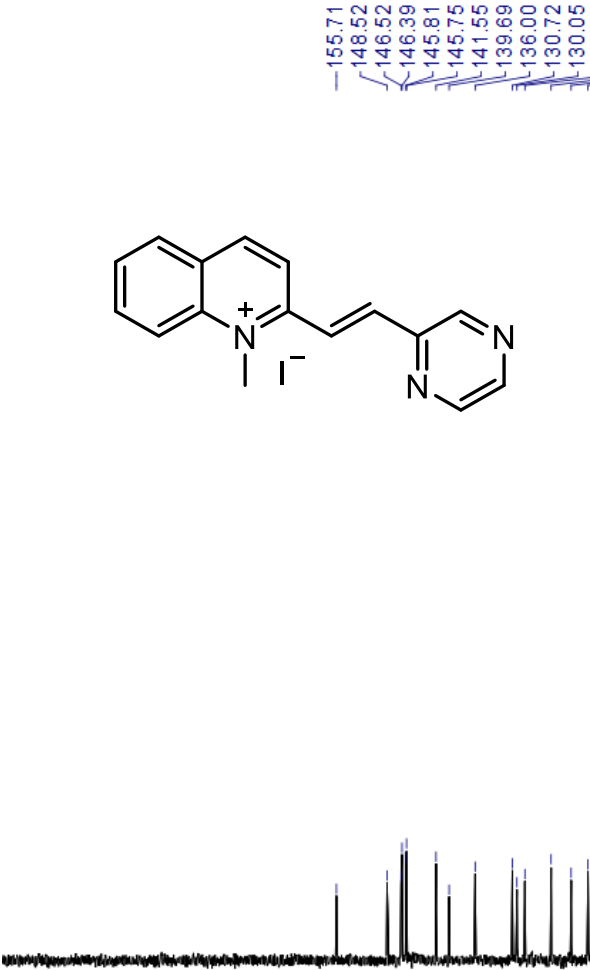


\section{HRMS of compound $\mathbf{3 b}$}

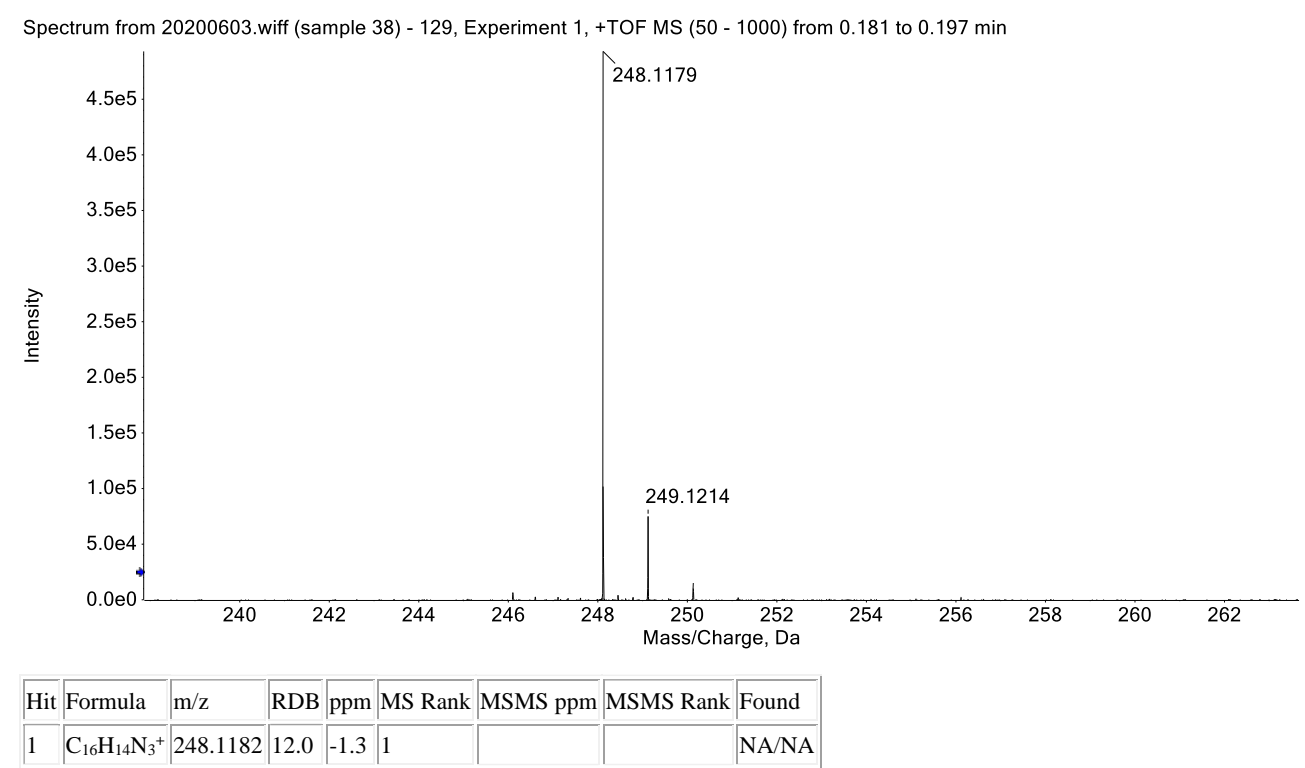

\section{HPLC analysis of $\mathbf{3 b}$}

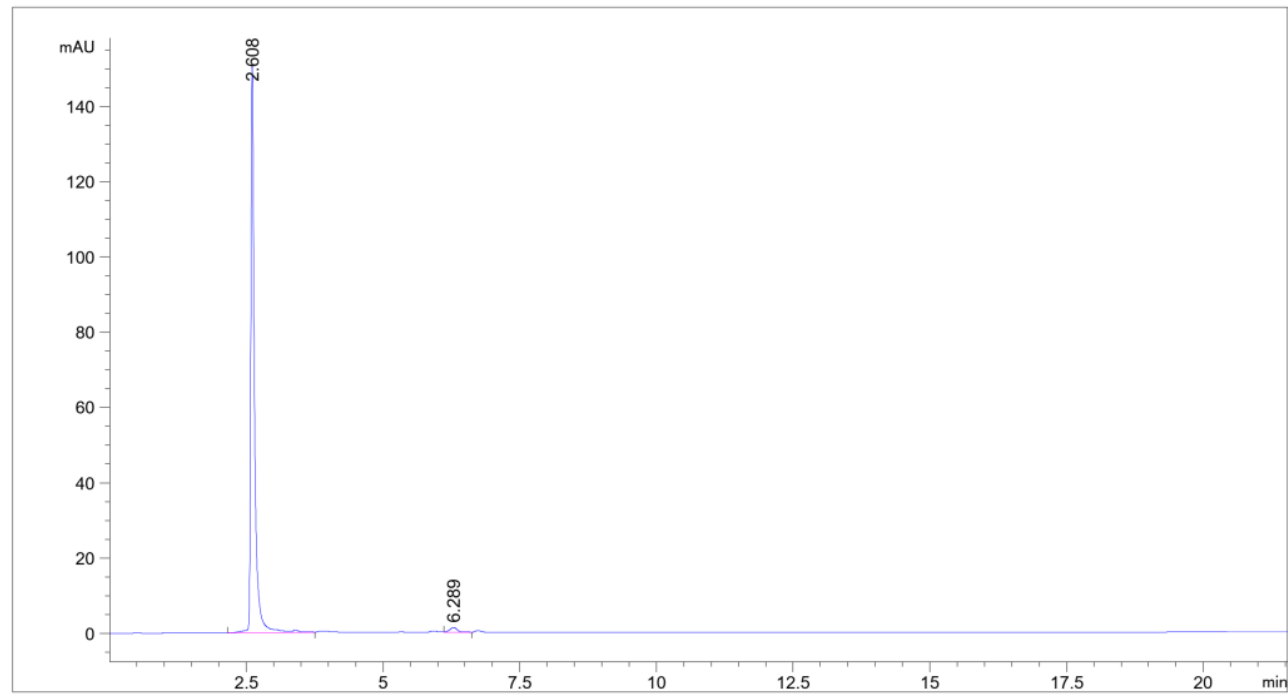

Area Percent Report

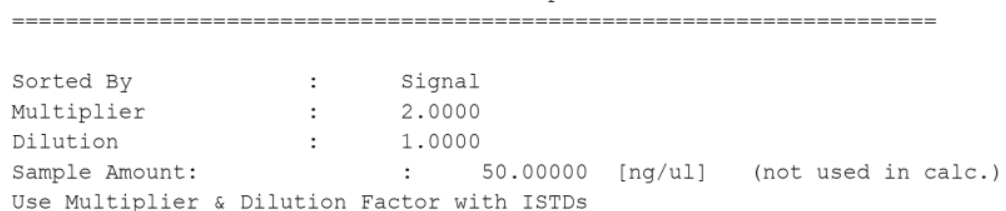

Signal 1: VWD1 A, Wavelength $=254 \mathrm{~nm}$

\begin{tabular}{|c|c|c|c|c|c|c|}
\hline $\begin{array}{c}\text { Peak } \\
\#\end{array}$ & $\begin{array}{c}\text { RetTime } \\
\text { [min] }\end{array}$ & Type & $\begin{array}{l}\text { Width } \\
\text { [min] }\end{array}$ & $\begin{array}{c}\text { Area } \\
{\left[\mathrm{mAU}^{*} \mathrm{~s}\right]}\end{array}$ & $\begin{array}{l}\text { Height } \\
\text { [MAU] }\end{array}$ & $\begin{array}{c}\text { Area } \\
\frac{\circ}{0}\end{array}$ \\
\hline 1 & 2.608 & BV $R$ & 0.0689 & 723.12524 & 150.86771 & 98.2090 \\
\hline 2 & 6.289 & VV R & 0.1534 & 13.18704 & 1.27774 & 1.7910 \\
\hline t & & & & 736.31228 & 152.14544 & \\
\hline
\end{tabular}


${ }^{1} \mathrm{H}-\mathrm{NMR}$ spectrum of compound $\mathbf{3 c}$

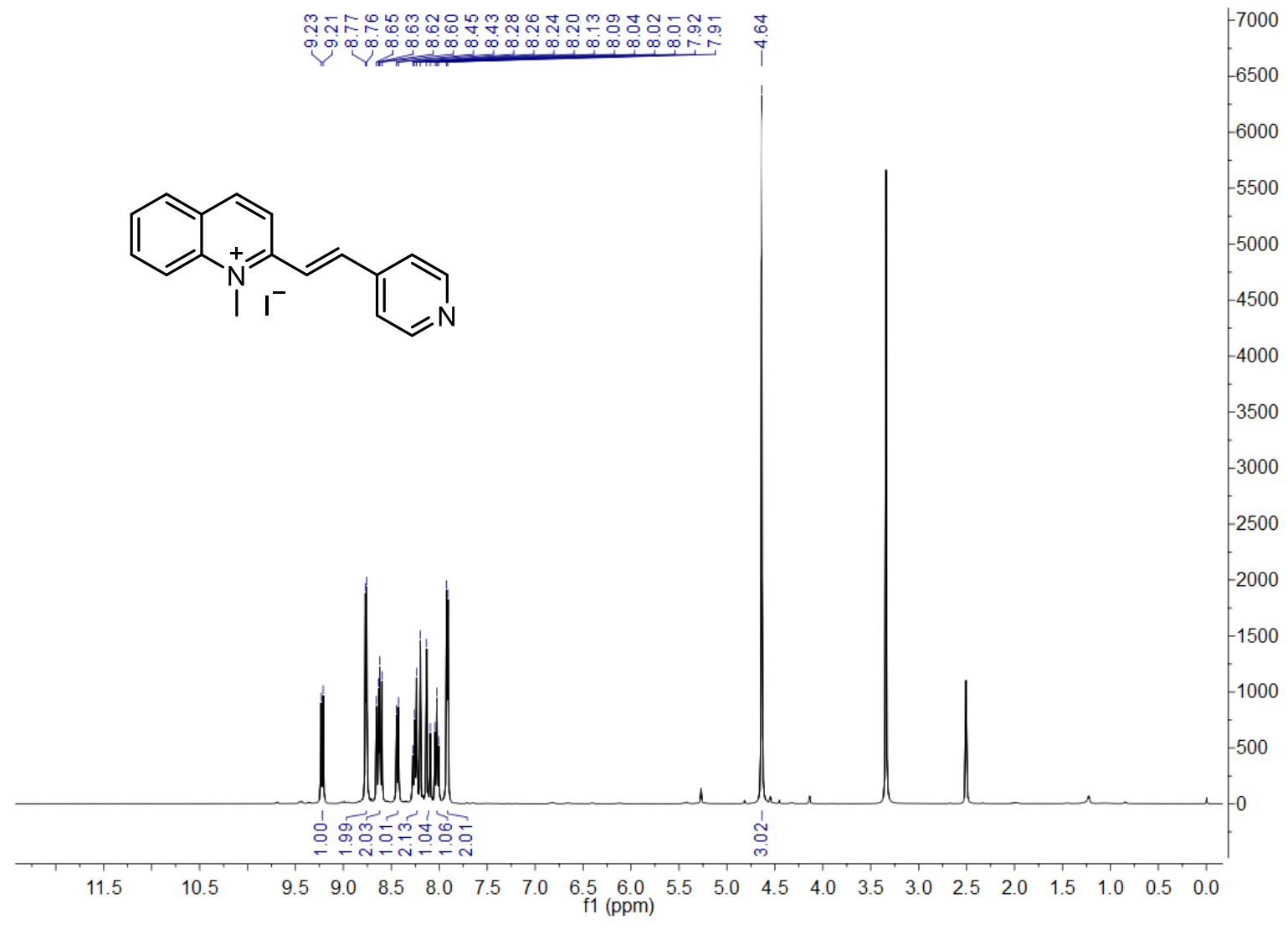

${ }^{13} \mathrm{C}-\mathrm{NMR}$ spectrum of compound $\mathbf{3 c}$ 


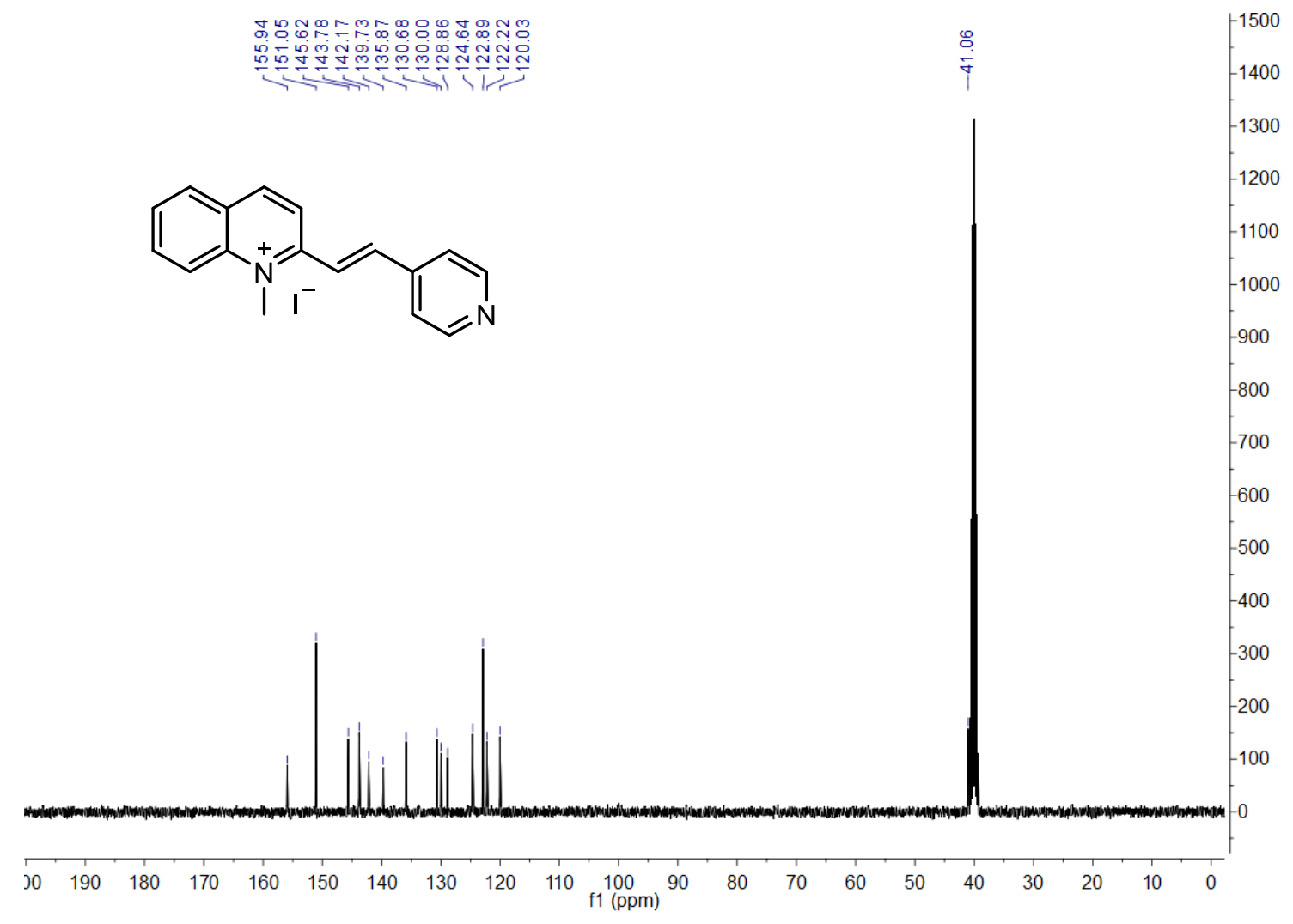

HRMS of compound 3c

Spectrum from 20200603.wiff (sample 39) - 172, Experiment 1, +TOF MS (50 - 1000) from 0.181 to $0.197 \mathrm{~min}$

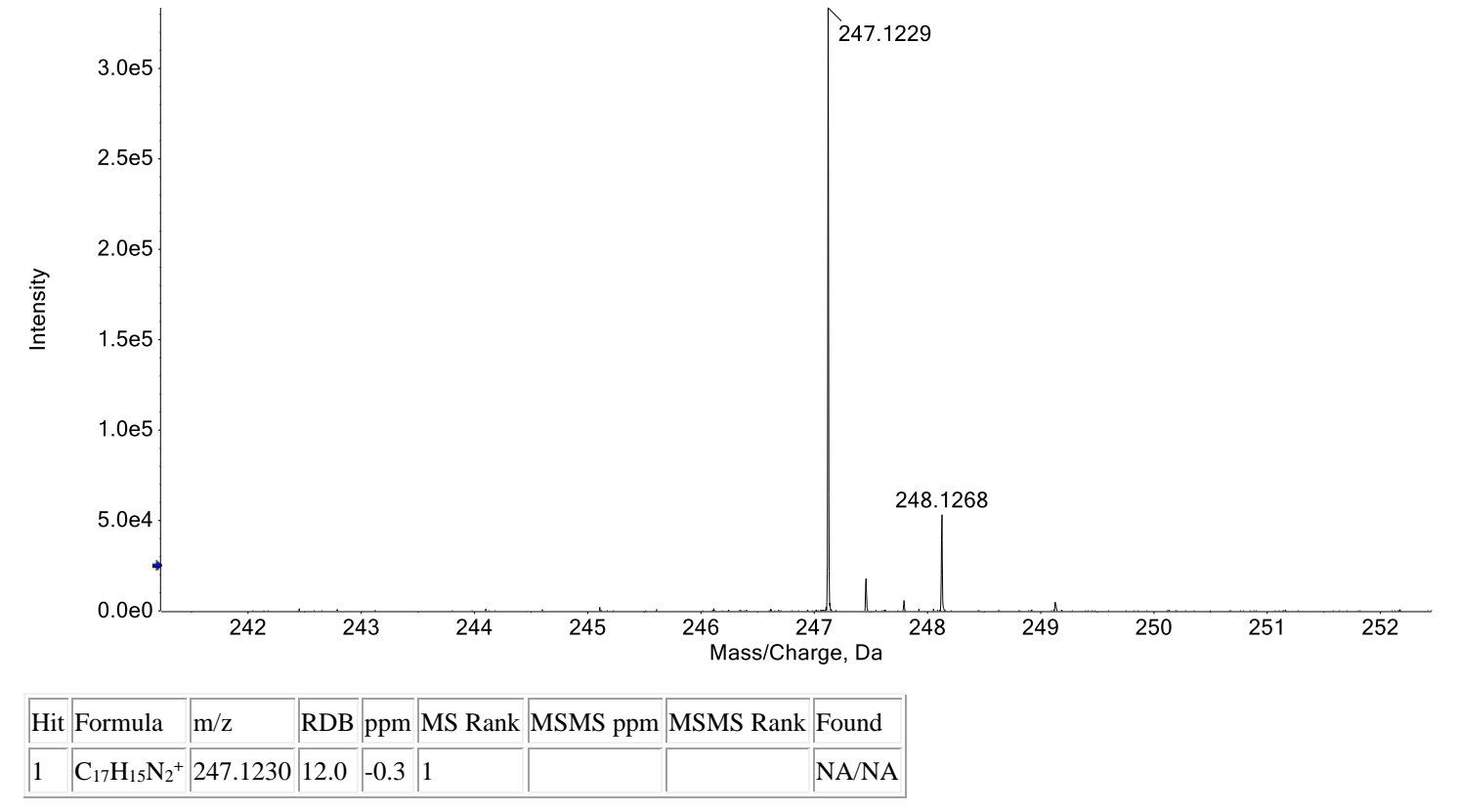


HPLC analysis of $\mathbf{3 c}$

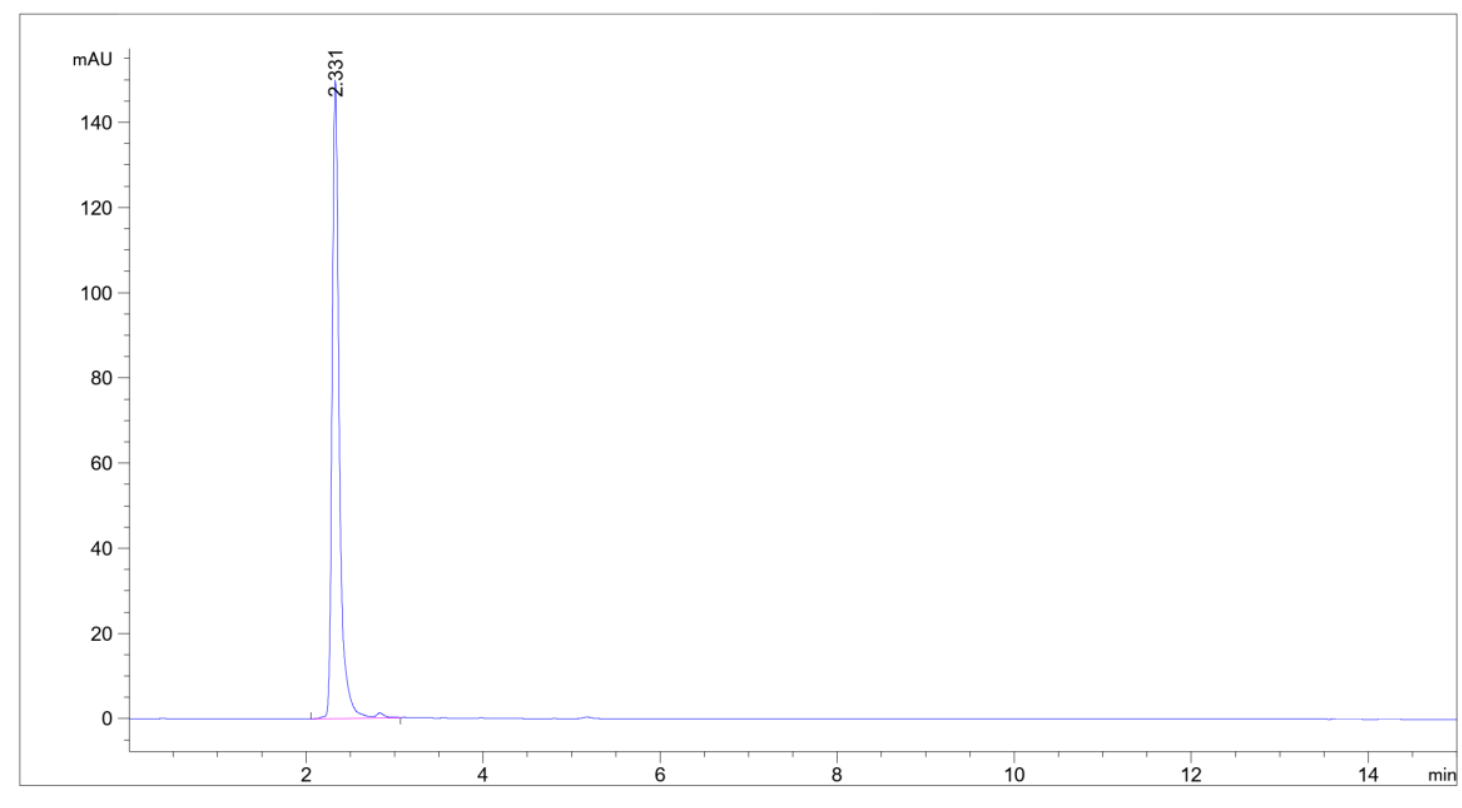

Area Percent Report

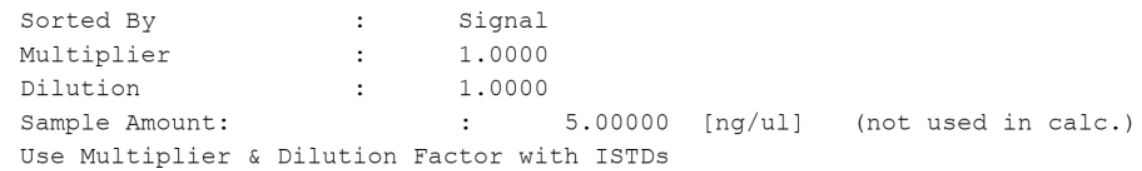

Signal 1: VWD1 A, Wavelength=254 nm

\begin{tabular}{|c|c|c|c|c|c|c|}
\hline $\begin{array}{c}\text { Peak } \\
\#\end{array}$ & $\begin{array}{l}\text { RetTime } \\
\text { [min] }\end{array}$ & Type & $\begin{array}{l}\text { Width } \\
\text { [min] }\end{array}$ & $\begin{array}{c}\text { Area } \\
{\left[\mathrm{mAU}{ }^{\star} \mathrm{s}\right]}\end{array}$ & $\begin{array}{l}\text { Height } \\
\text { [mAU] }\end{array}$ & $\begin{array}{c}\text { Area } \\
\frac{\circ}{\delta}\end{array}$ \\
\hline . & |----- & - & ----- & -------- & -------- & $|-------|$ \\
\hline 1 & 2.331 & BV $R$ & 0.0835 & 840.97778 & 149.87447 & 100.0000 \\
\hline & Is : & & & 840.97778 & 149.87447 & \\
\hline
\end{tabular}

${ }^{1} \mathrm{H}$-NMR spectrum of compound $\mathbf{4}$ 


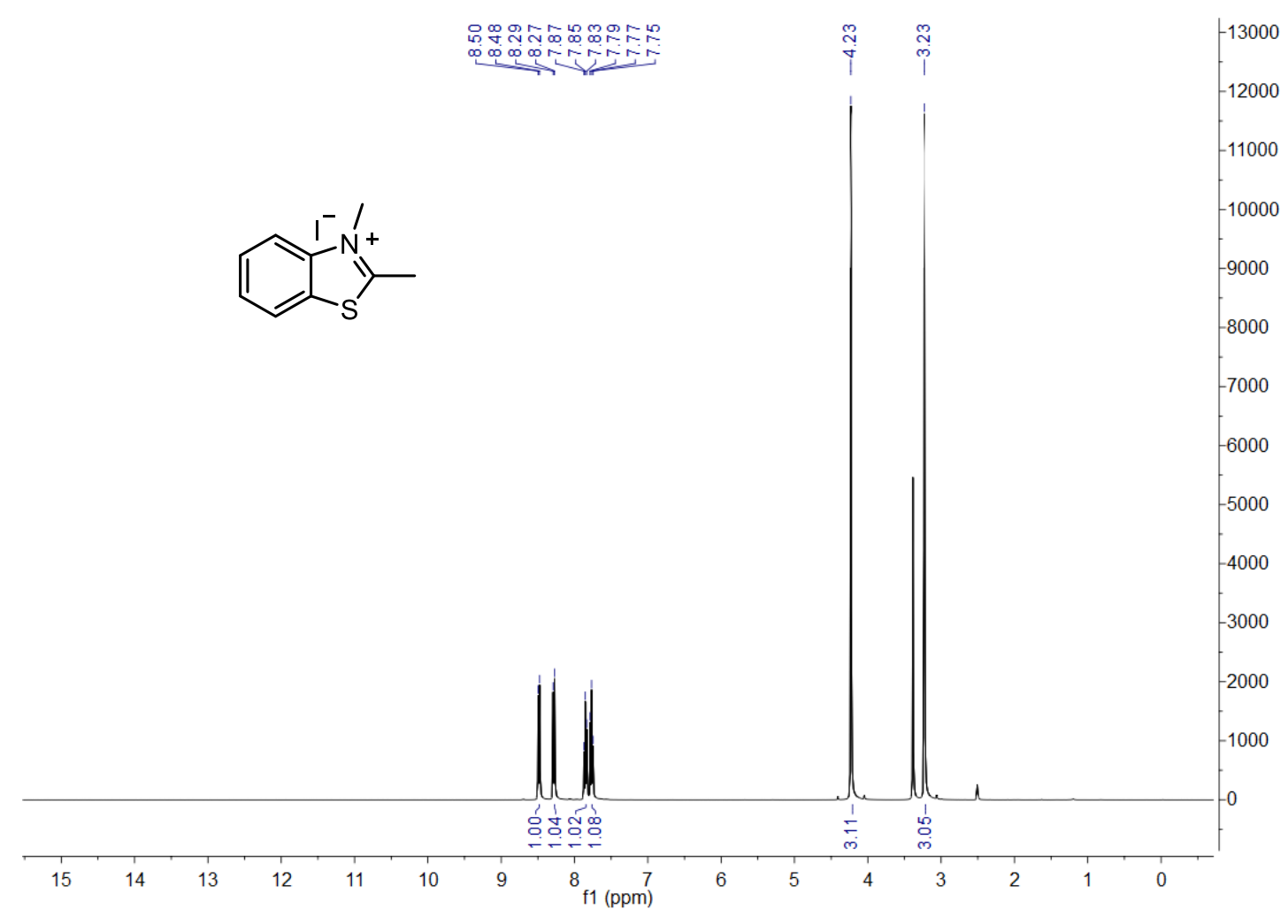

${ }^{13} \mathrm{C}-\mathrm{NMR}$ spectrum of compound 4

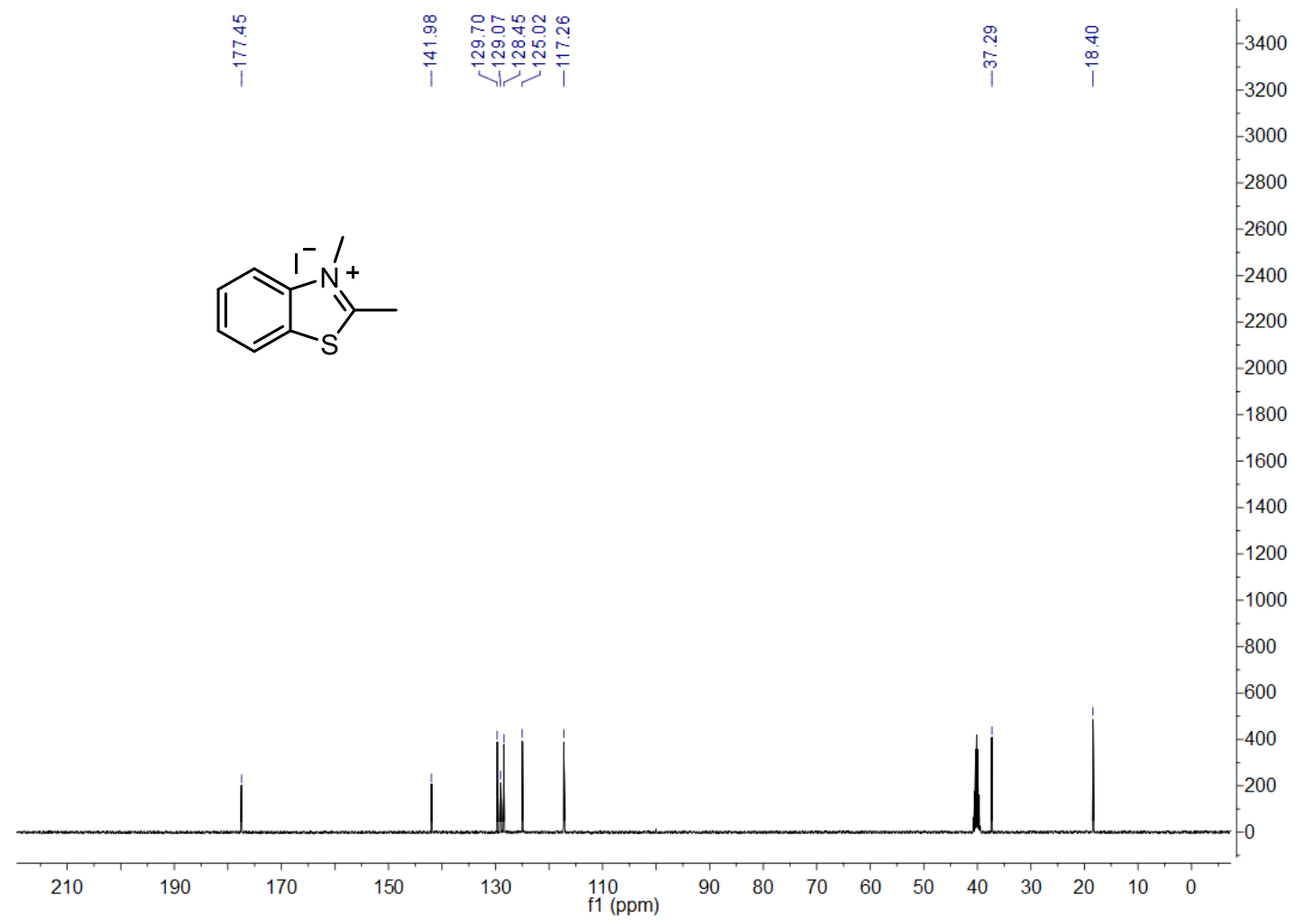




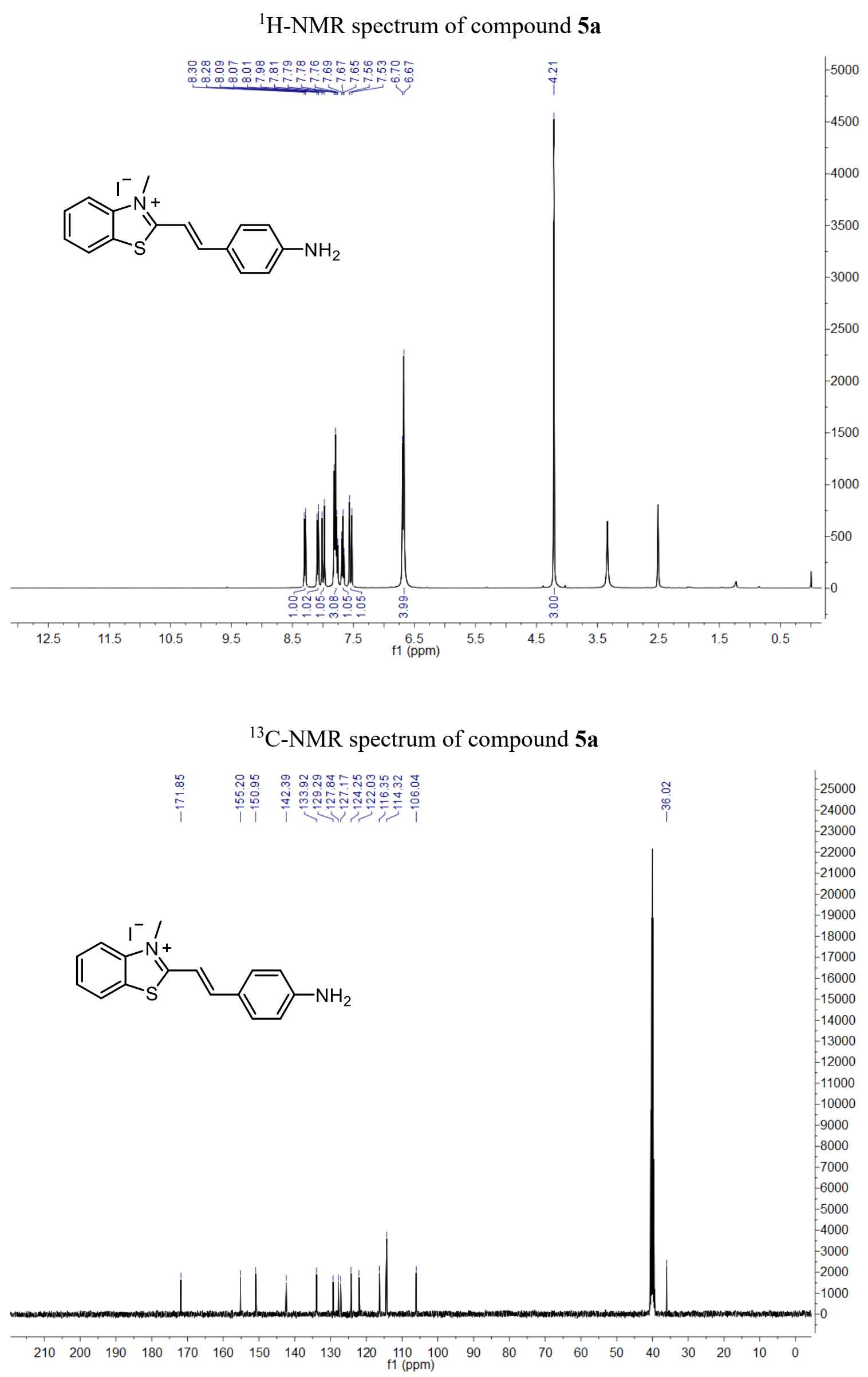


HRMS of compound $\mathbf{5 a}$

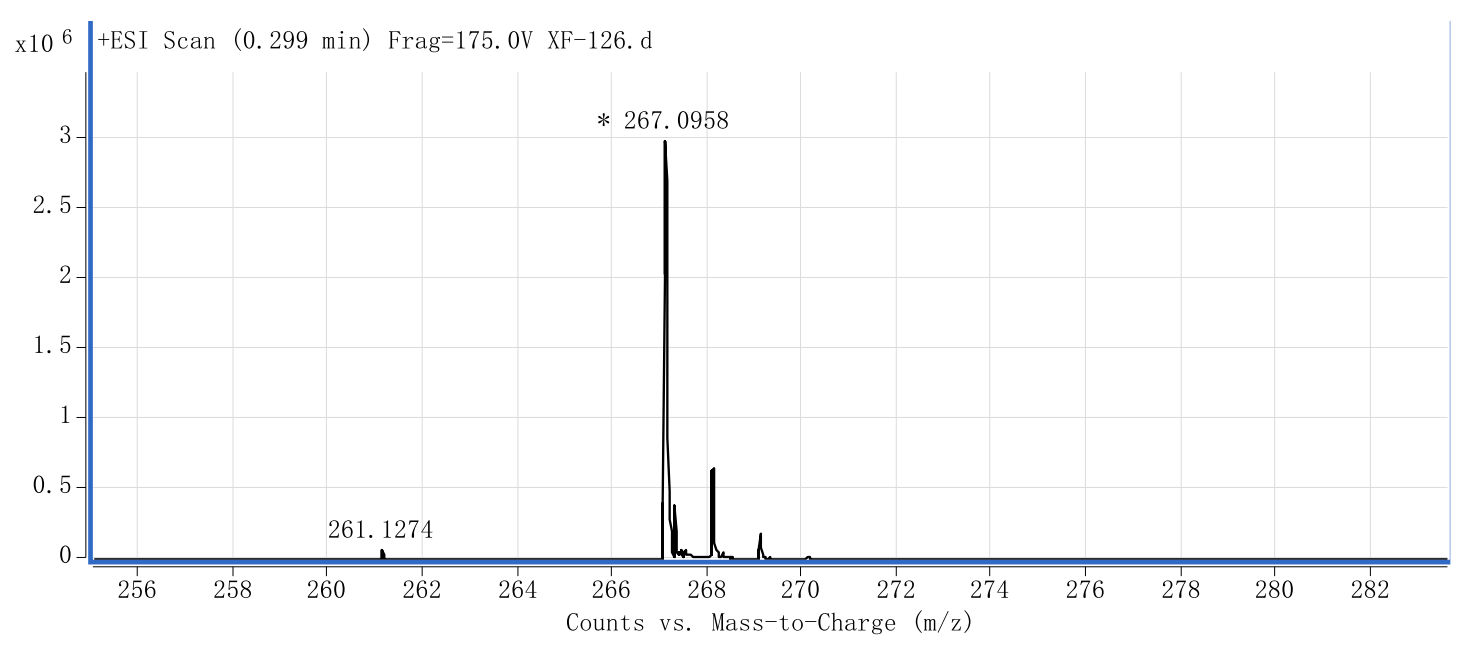

\begin{tabular}{|l|l|l|l|l|l|l|l|l|}
\hline Hit & Formula & Calc m/z & RDB & ppm & MS Rank & MSMS ppm & MSMS Rank & Found \\
\hline 1 & $\mathrm{C}_{16} \mathrm{H}_{15} \mathrm{~N}_{2} \mathrm{~S}^{+}$ & 267.0950 & 11.0 & 3.0 & 1 & & & NA/NA \\
\hline
\end{tabular}

HPLC analysis of $\mathbf{5 a}$

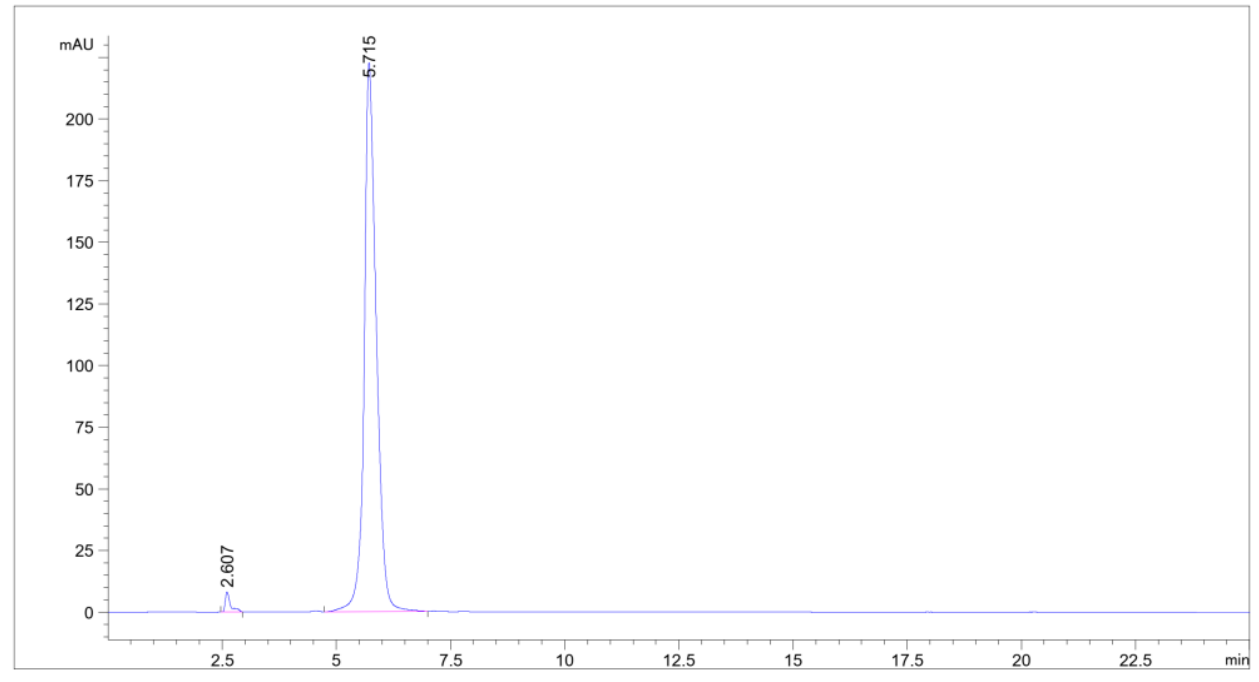

Area Percent Report

Sorted By : Signal

Multiplier : $\quad 1.0000$

Dilution : 1.0000

Use Multiplier \& Dilution Factor with ISTDs

Signal 1: VWD1 A, Wavelength $=254 \mathrm{~nm}$

\begin{tabular}{|c|c|c|c|c|c|c|}
\hline $\begin{array}{c}\text { Peak } \\
\#\end{array}$ & $\begin{array}{c}\text { RetTime } \\
\text { [min] }\end{array}$ & Type & $\begin{array}{l}\text { Width } \\
\text { [min] }\end{array}$ & $\begin{array}{c}\text { Area } \\
{\left[\mathrm{mAU}^{*} \mathrm{~s}\right]}\end{array}$ & $\begin{array}{l}\text { Height } \\
\text { [mAU] }\end{array}$ & $\begin{array}{c}\text { Area } \\
\frac{\circ}{0}\end{array}$ \\
\hline 1 & 2.607 & BV $R$ & 0.1189 & 63.62786 & 7.98946 & 1.5289 \\
\hline 2 & 5.715 & BB & 0.2719 & 4098.03760 & 222.51758 & 98.4711 \\
\hline
\end{tabular}

Totals: $\quad 4161.66546 \quad 230.50704$ 


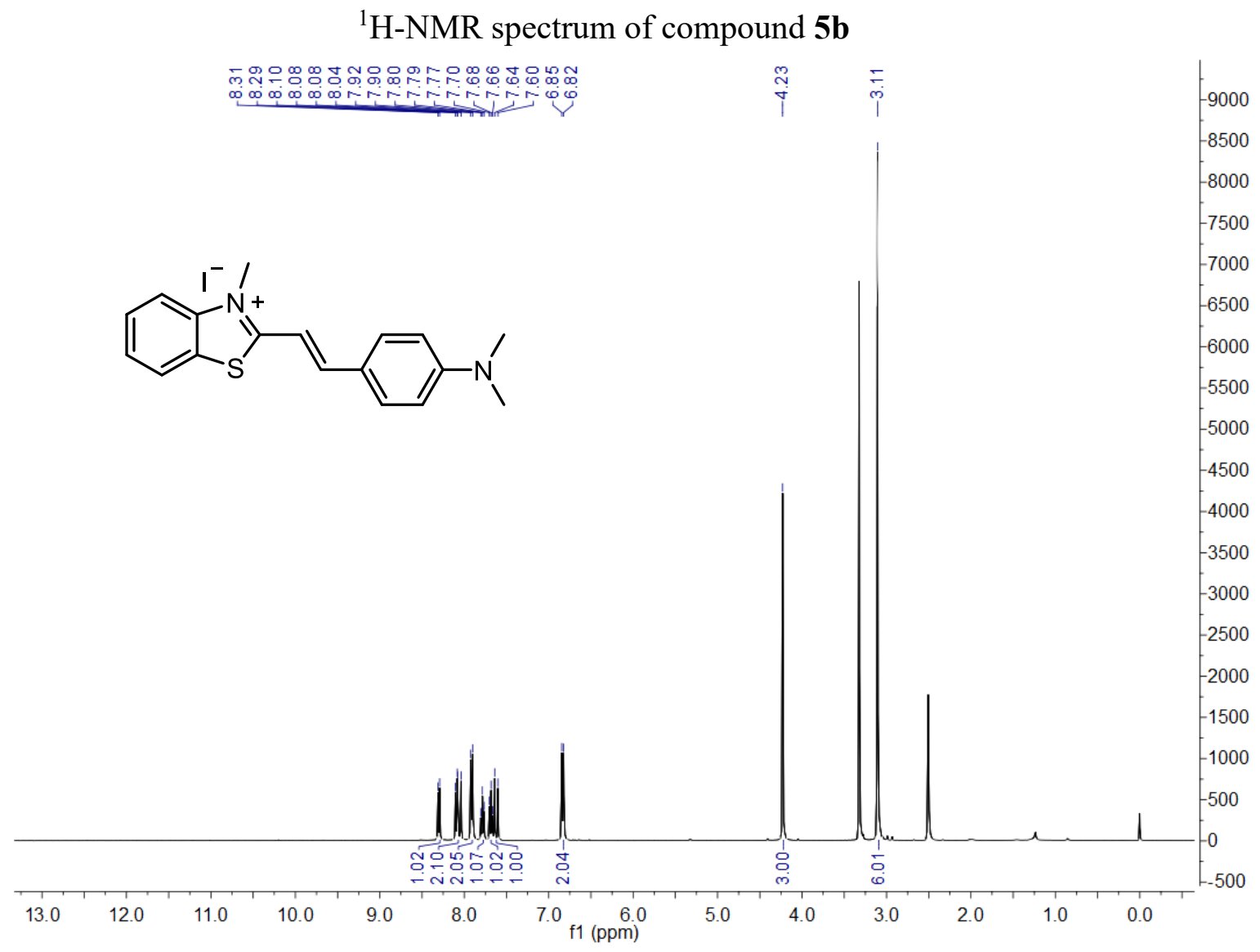

${ }^{13} \mathrm{C}-\mathrm{NMR}$ spectrum of compound $\mathbf{5 b}$ 


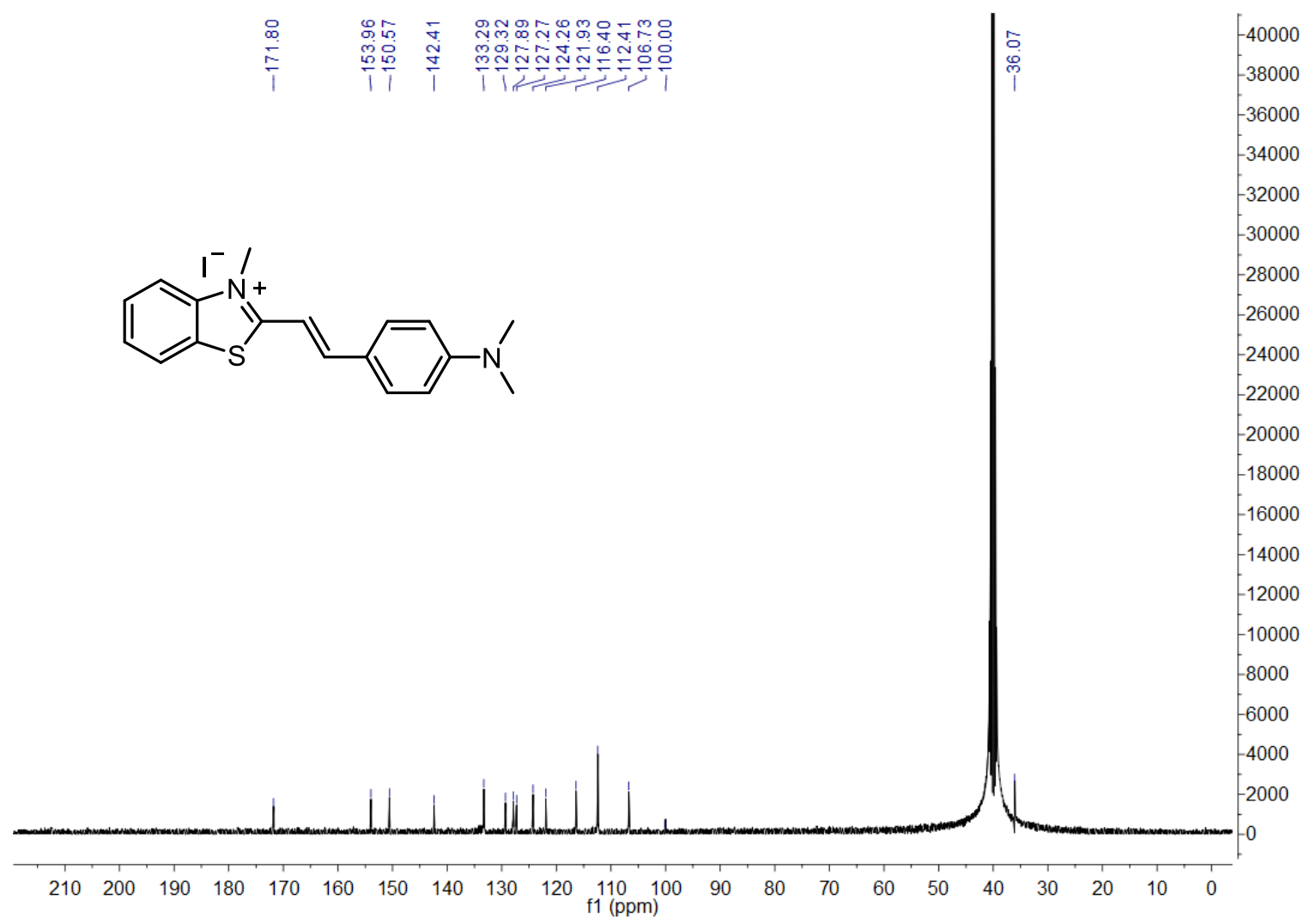

\section{HRMS of compound $\mathbf{5 b}$}

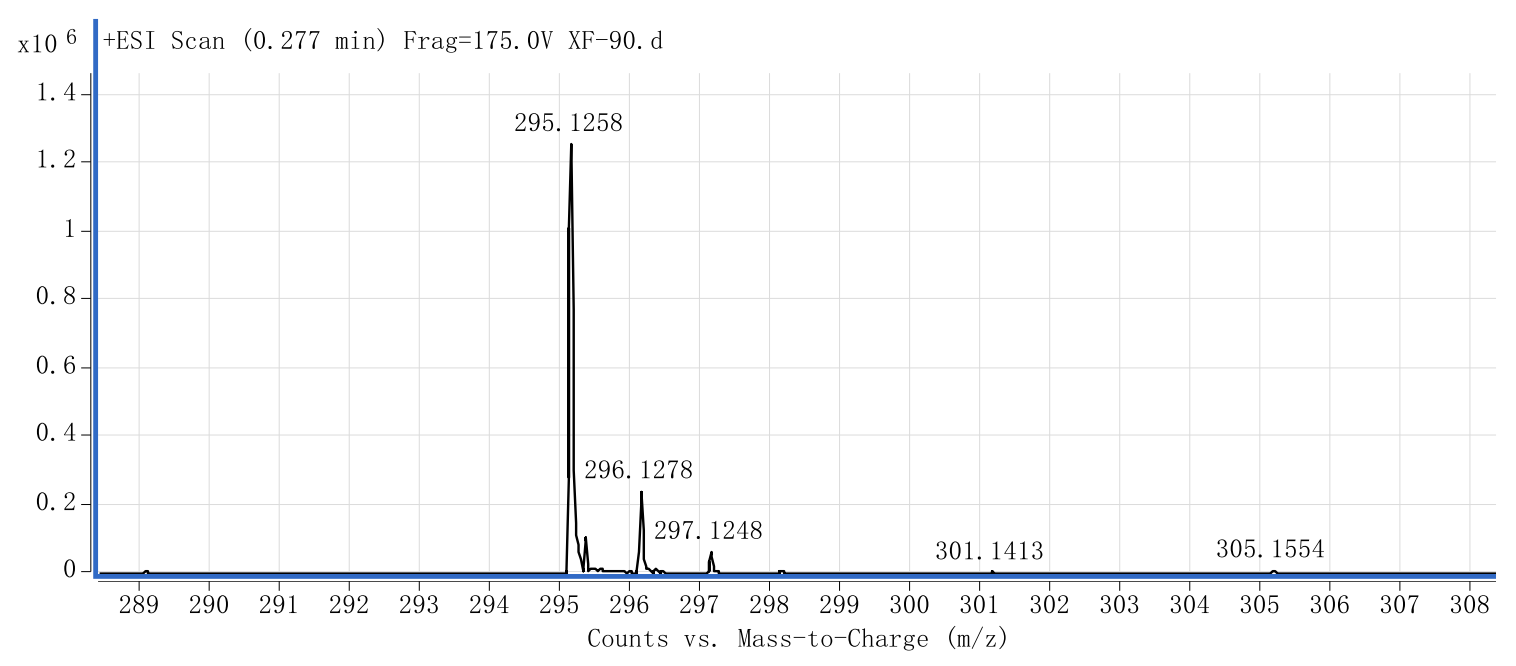

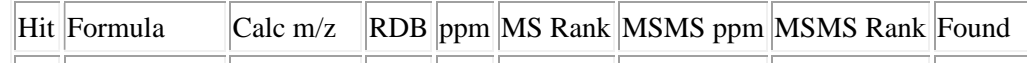

\begin{tabular}{|l|l|l|l|l|l|l|l|}
\hline 1 & $\mathrm{C}_{18} \mathrm{H}_{19} \mathrm{~N}_{2} \mathrm{~S}^{+}$ & 295.1263 & 11.0 & -1.6 & 1 & NA/NA
\end{tabular}

HPLC analysis of $\mathbf{5 b}$ 


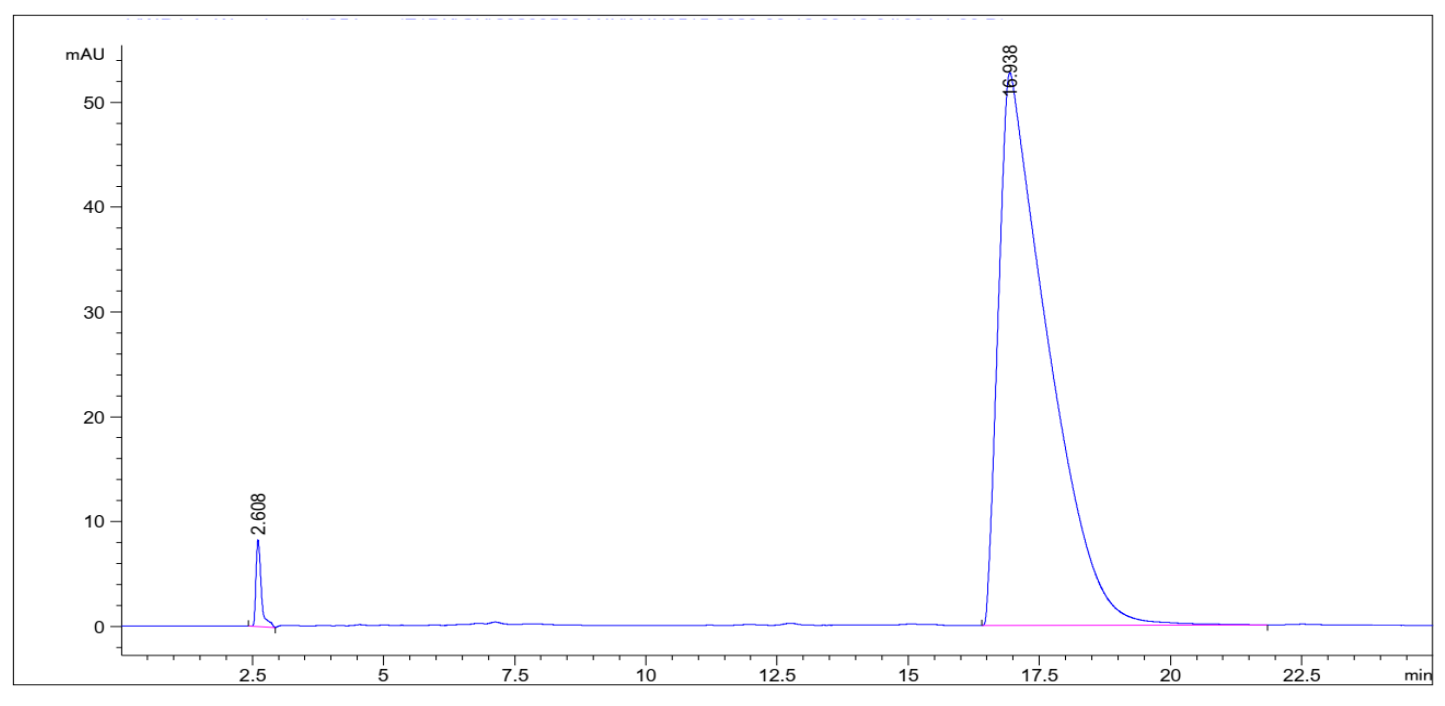

Area Percent Report

$\begin{array}{lll}\text { Sorted By } & : & \text { Signal } \\ \text { Multiplier } & : & 1.0000\end{array}$

$\begin{array}{lll}\text { Multiplier } & : & 1.0000 \\ \text { Dilution } & : & 1.0000\end{array}$

Use Multiplier \& Dilution Factor with ISTDs

Signal 1: VWDI A, Wavelength $=254 \mathrm{~nm}$

\begin{tabular}{|c|c|c|c|c|c|c|}
\hline $\begin{array}{c}\text { Peak } \\
\#\end{array}$ & $\begin{array}{c}\text { RetTime } \\
\text { [min] }\end{array}$ & Type & $\begin{array}{l}\text { Width } \\
\text { [min] }\end{array}$ & $\begin{array}{c}\text { Area } \\
{\left[\mathrm{mAU}^{\star} \mathrm{S}\right]}\end{array}$ & $\begin{array}{l}\text { Height } \\
\text { [mAU] }\end{array}$ & $\begin{array}{c}\text { Area } \\
\frac{\circ}{8}\end{array}$ \\
\hline & & & & |---------- & -------- & -------1 \\
\hline 1 & 2.608 & $\mathrm{BB}$ & 0.1078 & 58.07257 & 8.28707 & 1.6611 \\
\hline 2 & 16.938 & $\mathrm{BB}$ & 0.8874 & 3437.93872 & 52.74504 & 98.3389 \\
\hline Total & IS : & & & 3496.01129 & 61.03212 & \\
\hline
\end{tabular}

${ }^{1} \mathrm{H}-\mathrm{NMR}$ spectrum of $\mathbf{5 c}$ 

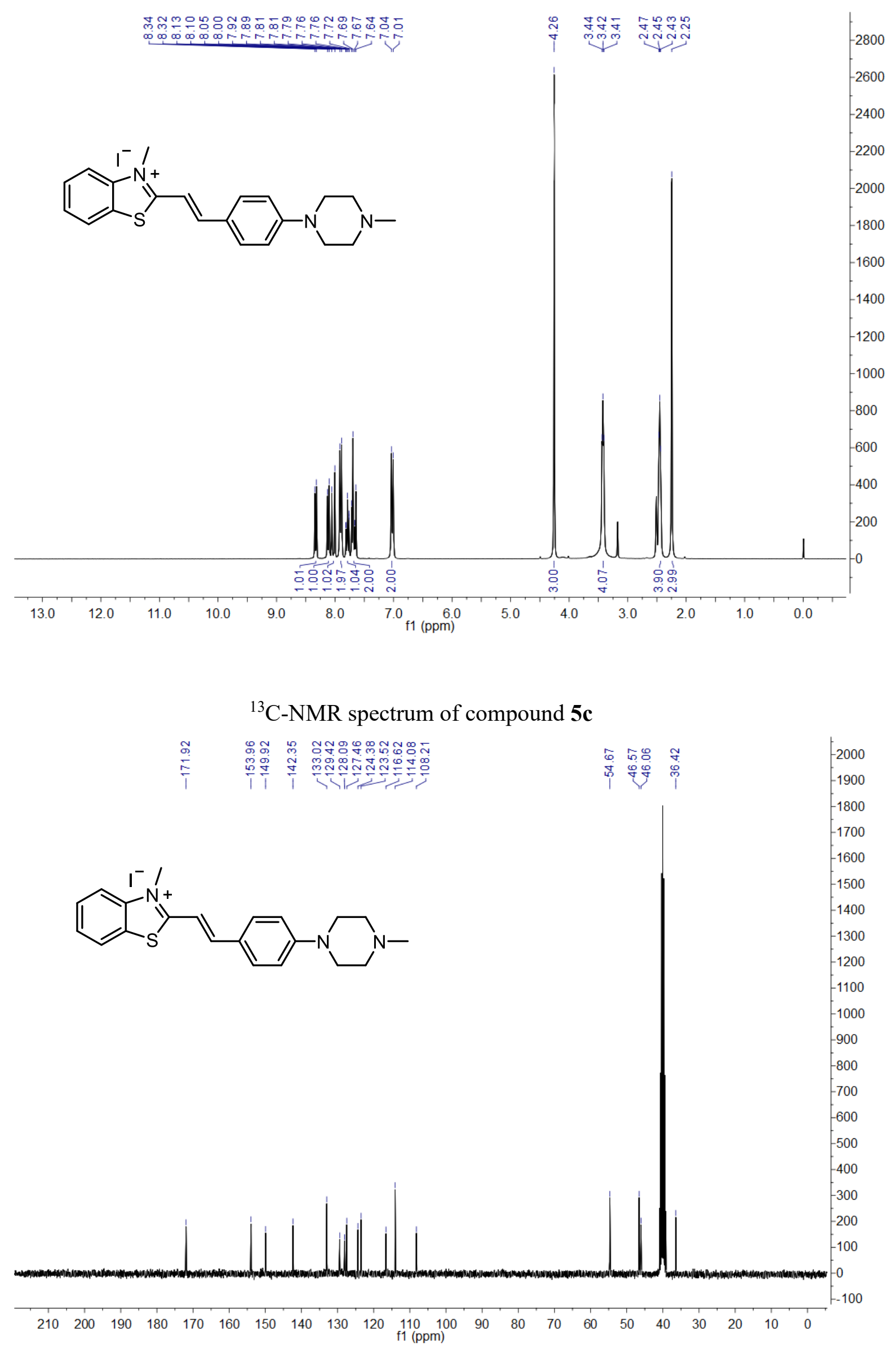
HRMS of compound 5c

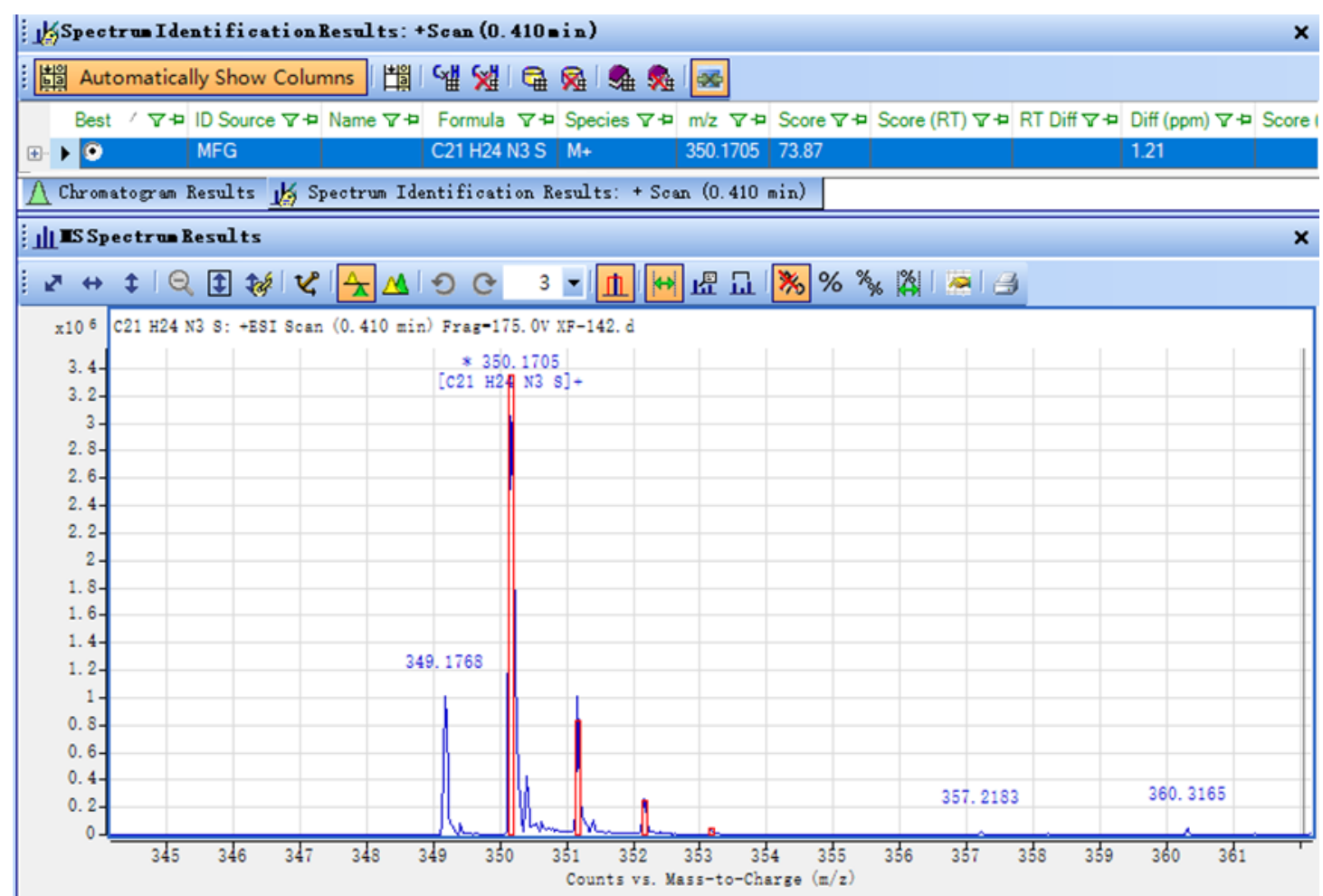


HPLC analysis of $\mathbf{5 c}$

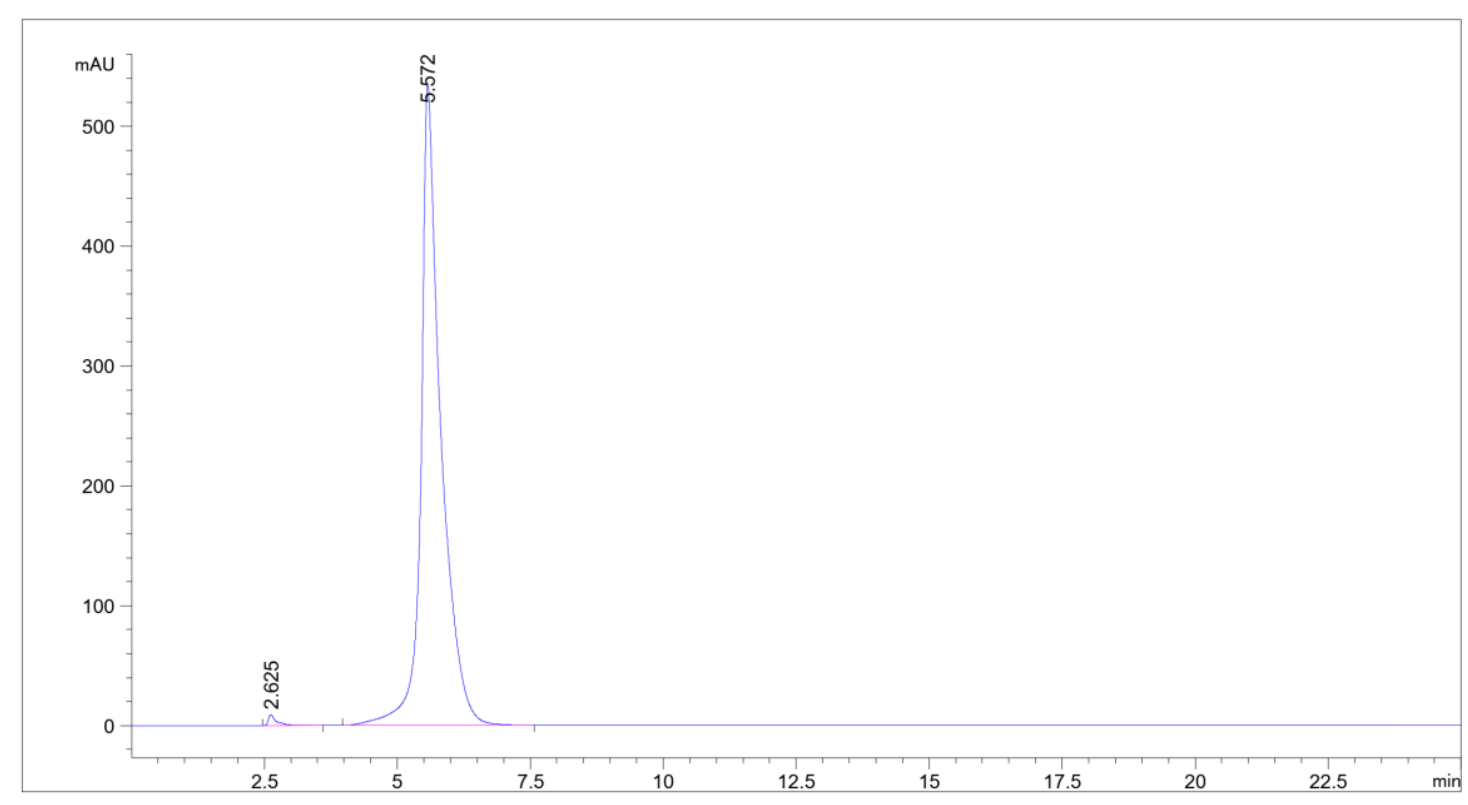

Area Percent Report

$\begin{array}{lll}\text { Sorted By } & : & \text { Signal } \\ \text { Multiplier } & : & 1.0000 \\ \text { Dilution } & : & 1.0000\end{array}$

Use Multiplier \& Dilution Factor with ISTDs

Signal 1: VWD1 A, Wavelength=254 nm

\begin{tabular}{|c|c|c|c|c|c|c|}
\hline $\begin{array}{c}\text { Peak } \\
\quad \#\end{array}$ & $\begin{array}{c}\text { RetTime } \\
\text { [min] }\end{array}$ & Type & $\begin{array}{l}\text { Width } \\
\text { [min] }\end{array}$ & $\begin{array}{c}\text { Area } \\
{\left[\mathrm{mAU}^{*} \mathrm{~S}\right]}\end{array}$ & $\begin{array}{l}\text { Height } \\
\text { [mAU] }\end{array}$ & $\begin{array}{c}\text { Area } \\
\%\end{array}$ \\
\hline & ---- & & ---- & |---------- & $----------\mid$ & ------- \\
\hline 1 & 2.625 & BB & 0.1545 & 97.01402 & 8.94430 & 0.6922 \\
\hline 2 & 5.572 & $\mathrm{BB}$ & 0.3563 & $1.39175 \mathrm{e} 4$ & 533.51563 & 99.3078 \\
\hline Total & : & & & $1.40145 e 4$ & 542.45992 & \\
\hline
\end{tabular}




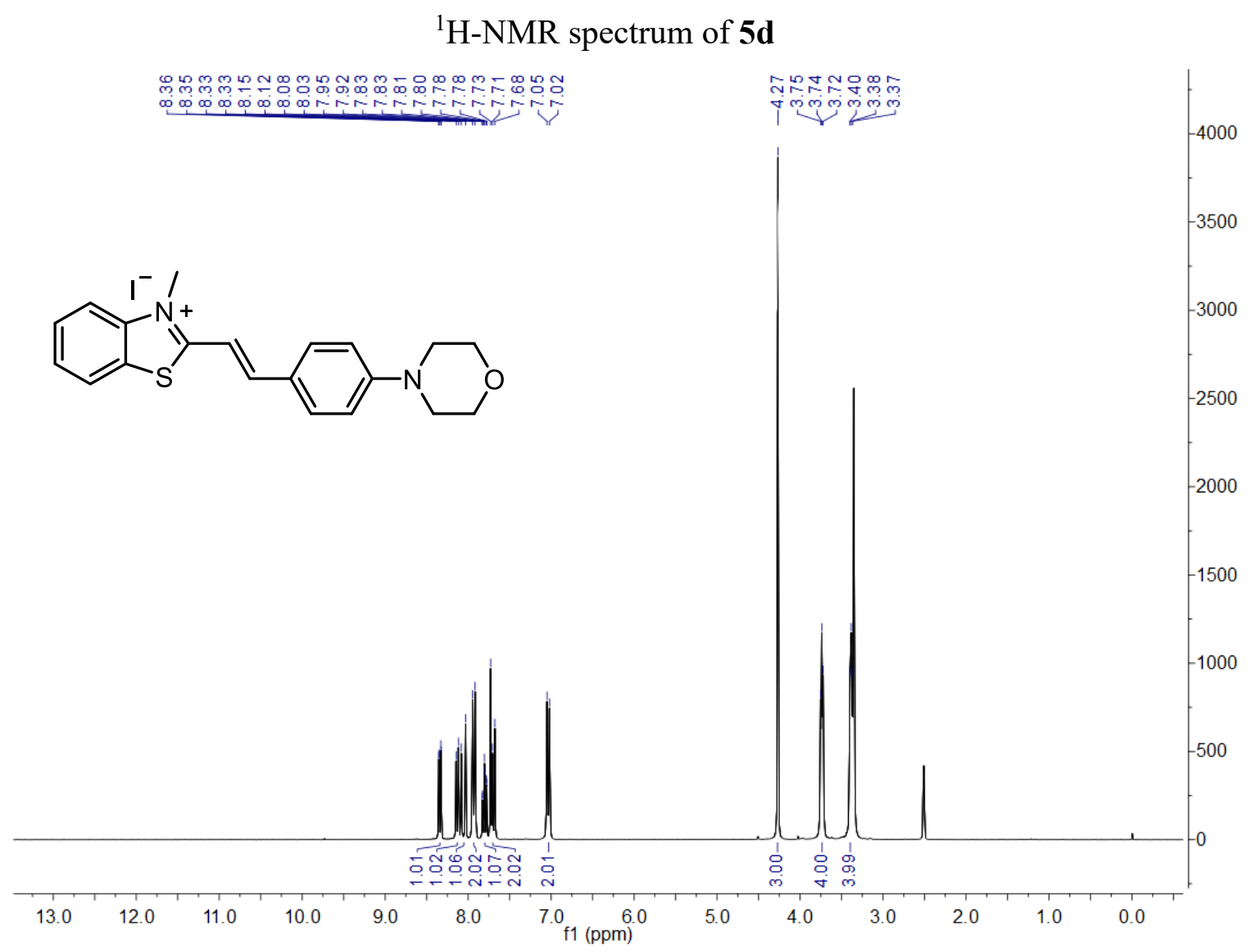

${ }^{13} \mathrm{C}-\mathrm{NMR}$ spectrum of compound $\mathbf{5 d}$ 


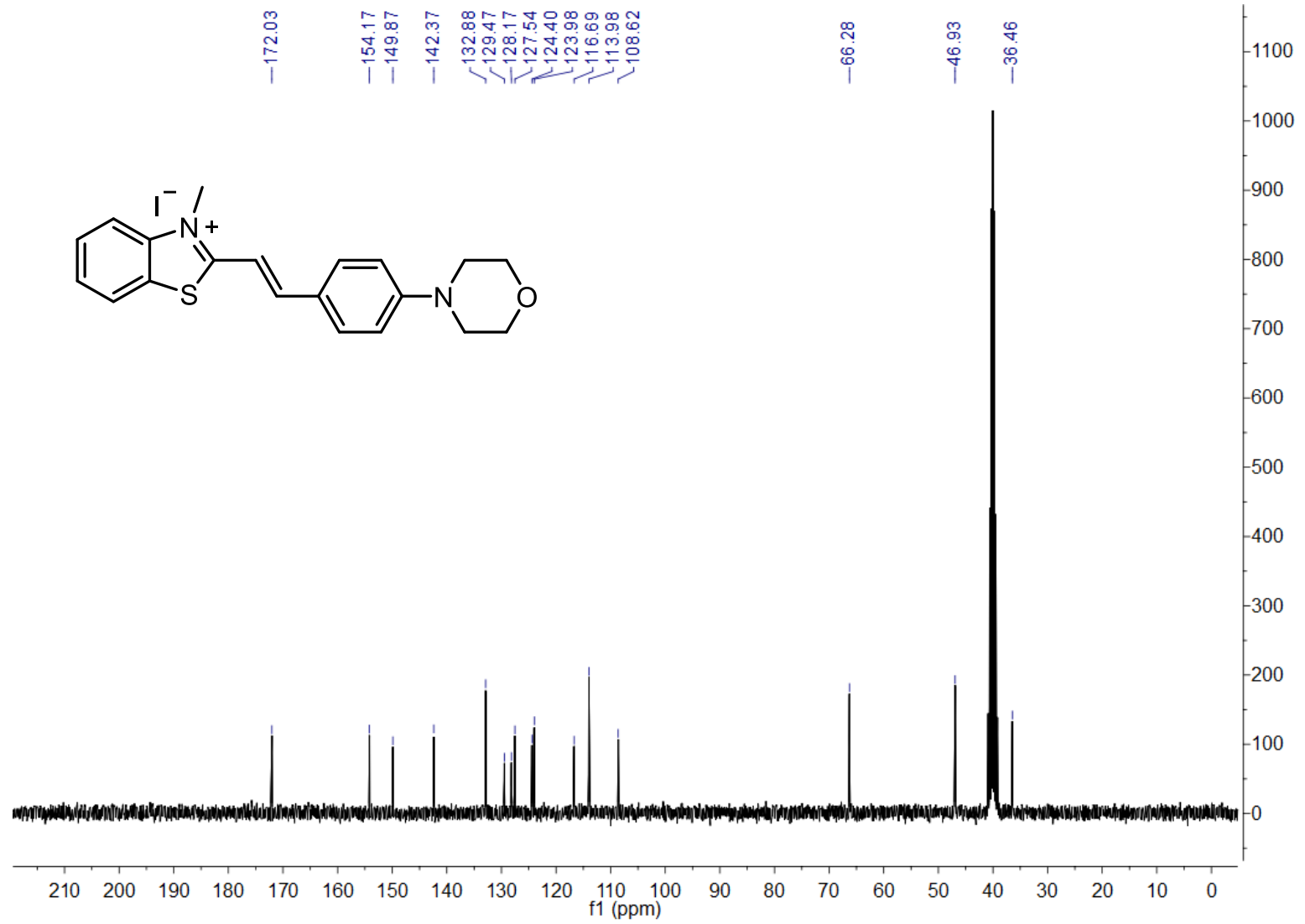

HRMS of compound 5d

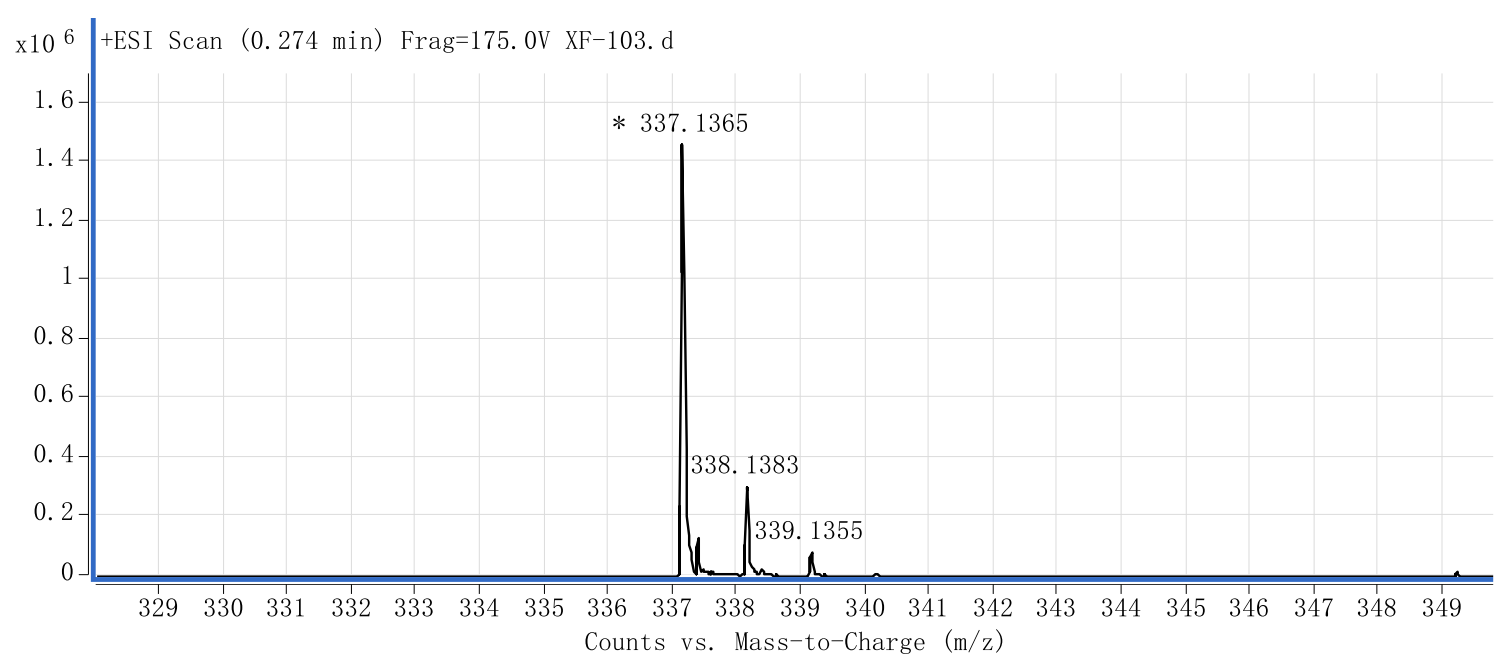

\begin{tabular}{|l|l|l|l|l|l|l|l|l|}
\hline Hit & Formula & Calc m/z & RDB & ppm & MS Rank & MSMS ppm & MSMS Rank & Found \\
\hline 1 & $\mathrm{C}_{20} \mathrm{H}_{21} \mathrm{~N}_{2} \mathrm{OS}^{+}$ & 337.1369 & 12.0 & -0.9 & 1 & & & NA/NA \\
\hline
\end{tabular}

HPLC analysis of $\mathbf{5 d}$ 


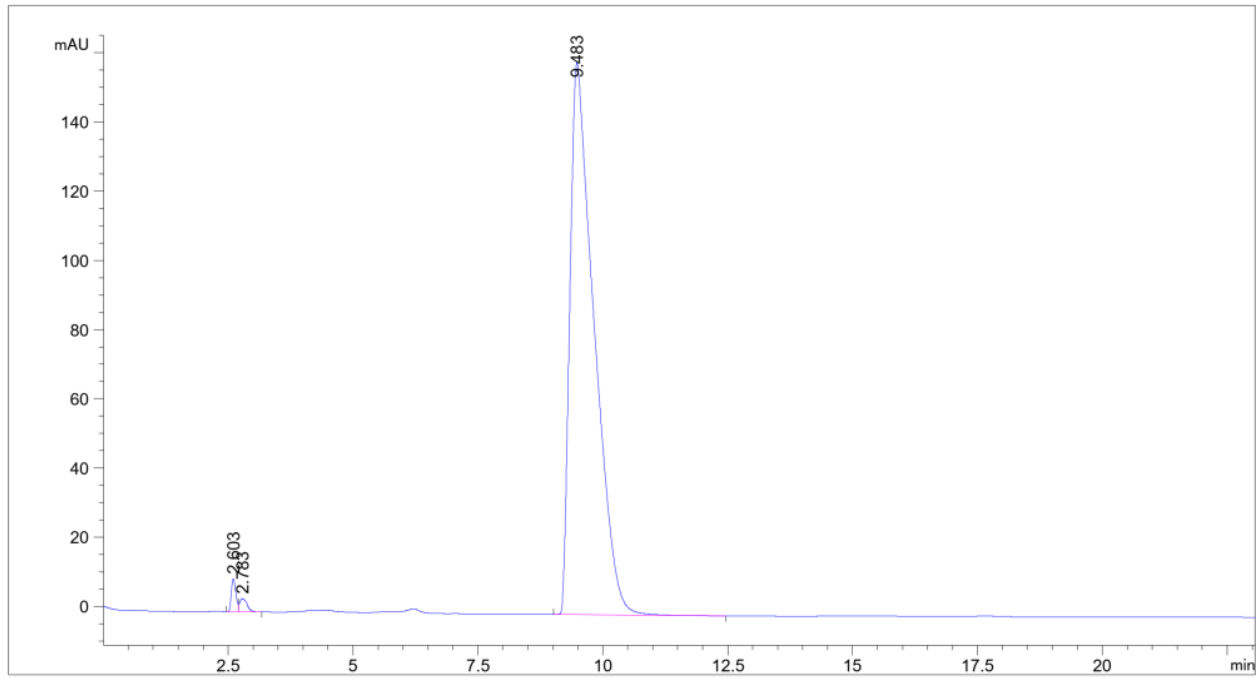

Area Percent Report

$\begin{array}{llll}\text { Sorted By } & : & \text { Signal } & \\ \text { Multiplier } & : & 1.0000 \\ \text { Dilution } & : & 1.0000 \\ \text { Sample Amount: } & & : \quad 10.00000 \quad \text { [ng/ul] (not used in calc.) }\end{array}$

Use Multiplier \& Dilution Factor with ISTDs

Signal 1: VWD1 A, Wavelength $=254 \mathrm{~nm}$

\begin{tabular}{|c|c|c|c|c|c|c|}
\hline $\begin{array}{c}\text { Peak } \\
\quad \#\end{array}$ & $\begin{array}{c}\text { RetTime } \\
\text { [min] }\end{array}$ & Type & $\begin{array}{l}\text { Width } \\
\text { [min] }\end{array}$ & $\begin{array}{c}\text { Area } \\
{\left[\mathrm{mAU}^{\star} \mathrm{s}\right]}\end{array}$ & $\begin{array}{l}\text { Height } \\
\text { [mAU] }\end{array}$ & $\begin{array}{c}\text { Area } \\
\frac{\circ}{0}\end{array}$ \\
\hline & & & 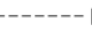 & $-x-1$ & - & ------- \\
\hline 1 & 2.603 & BV & 0.0998 & 60.17568 & 9.51525 & 1.1103 \\
\hline 2 & 2.783 & VB & 0.1417 & 38.62078 & 3.73314 & 0.7126 \\
\hline 3 & 9.483 & BB & 0.4724 & 5321.01367 & 159.44095 & 98.1771 \\
\hline
\end{tabular}

Totals :

$5419.81014 \quad 172.68934$

${ }^{1} \mathrm{H}-\mathrm{NMR}$ spectrum of $\mathbf{5 e}$ 


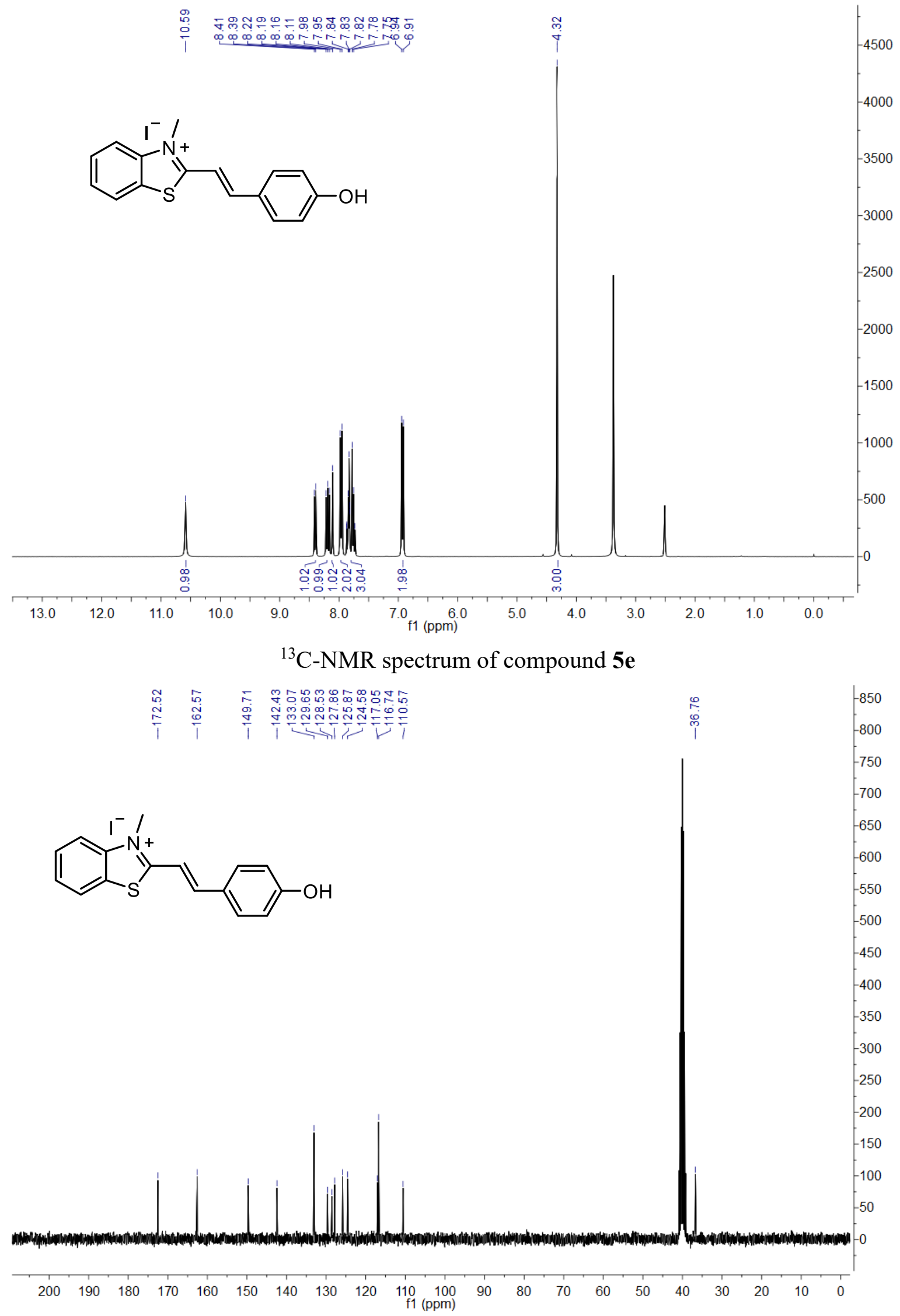




\section{HRMS of compound $\mathbf{5 e}$}

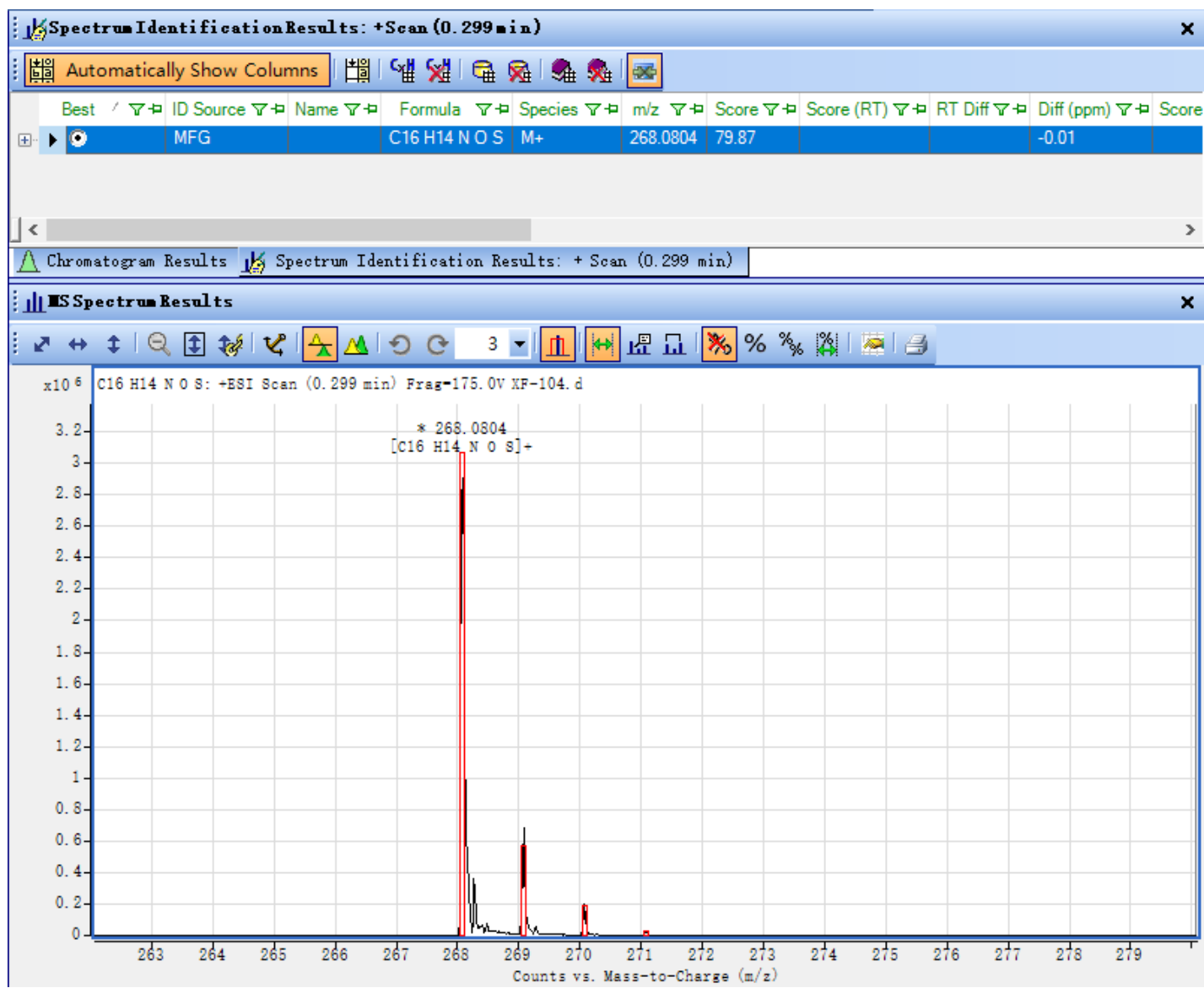


HPLC analysis of $\mathbf{5 e}$

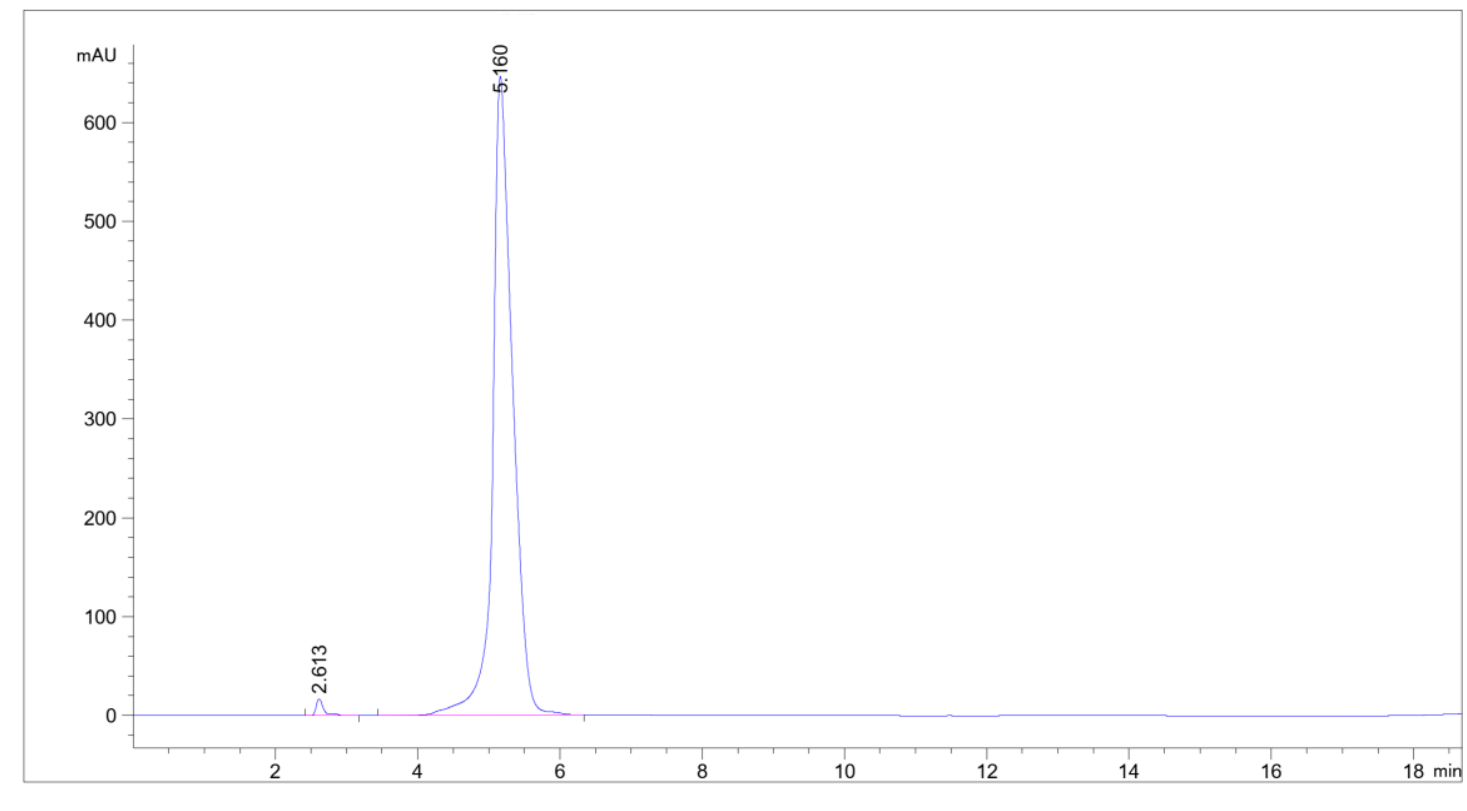

Area Percent Report

\begin{tabular}{|c|c|c|c|c|}
\hline Sorted By & : & Signal & & \\
\hline Multiplier & : & 1.0000 & & \\
\hline Dilution & : & 1.0000 & & \\
\hline Sample Amount: & & $: \quad 10.00000$ & [ng/ul] & (not used in calc.) \\
\hline
\end{tabular}

Signal 1: VWD1 A, Wavelength=254 nm

\begin{tabular}{|c|c|c|c|c|c|c|}
\hline $\begin{array}{c}\text { Peak } \\
\quad \#\end{array}$ & $\begin{array}{c}\text { RetTime } \\
\text { [min] }\end{array}$ & Type & $\begin{array}{l}\text { Width } \\
\text { [min] }\end{array}$ & $\begin{array}{c}\text { Area } \\
{\left[\mathrm{mAU}^{\star} \mathrm{S}\right]}\end{array}$ & $\begin{array}{l}\text { Height } \\
\text { [mAU] }\end{array}$ & $\begin{array}{c}\text { Area } \\
\frac{\%}{\circ}\end{array}$ \\
\hline---1 & ------- & $|----|$ & $-------\mid$ & $|----------|$ & $----------\mid$ & --------1 \\
\hline 1 & 2.613 & $B V R$ & 0.1151 & 126.54945 & 16.75034 & 0.9944 \\
\hline 2 & 5.160 & $\mathrm{BB}$ & 0.2824 & $1.25993 \mathrm{e} 4$ & 646.37433 & 99.0056 \\
\hline
\end{tabular}

$\begin{array}{lll}\text { Totals : } & 1.27259 \mathrm{e} 4 & 663.12467\end{array}$

${ }^{1} \mathrm{H}$ NMR spectrum of compound $\mathbf{5 f}$ 

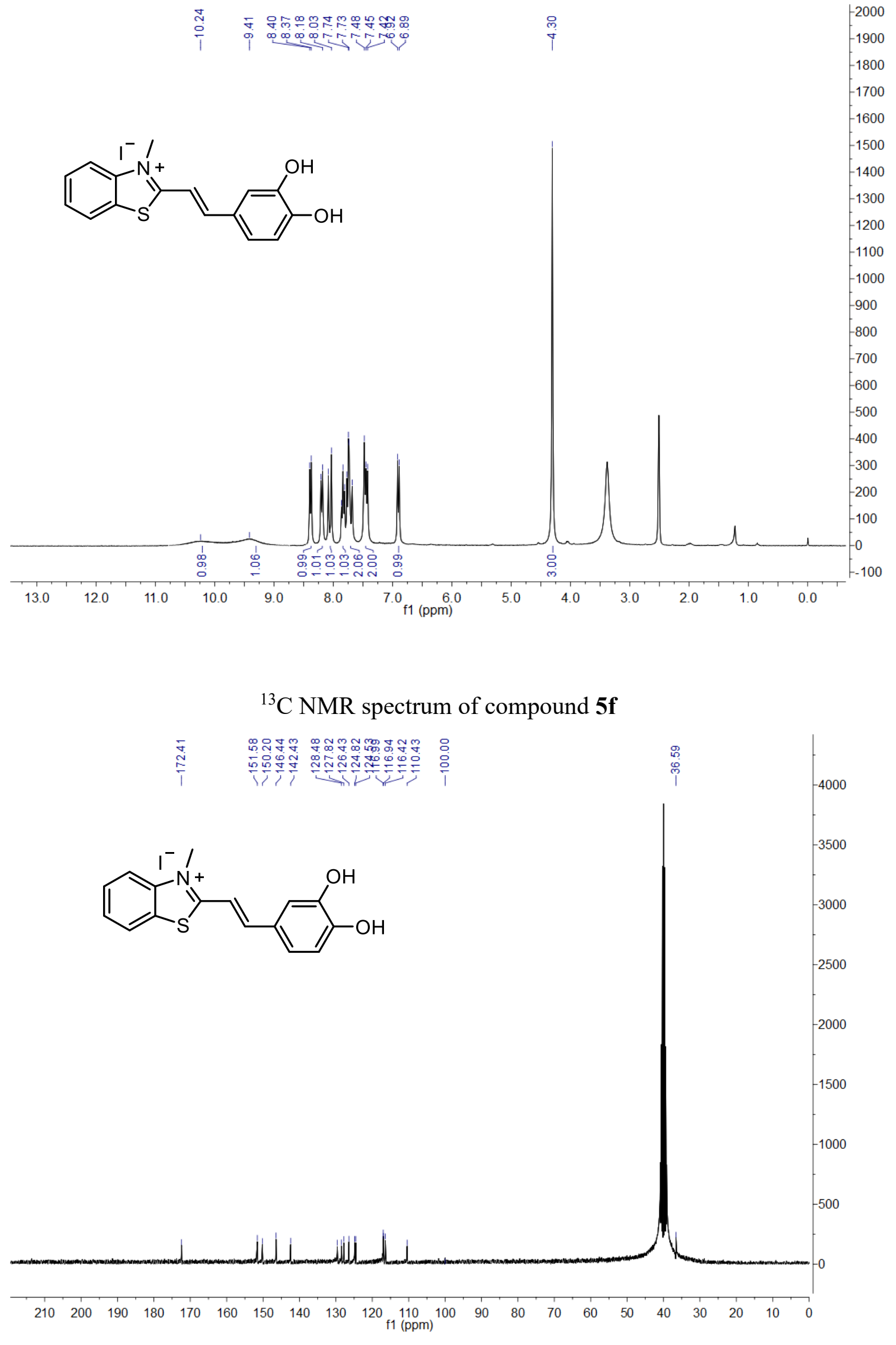

HRMS of compound $\mathbf{5 f}$ 


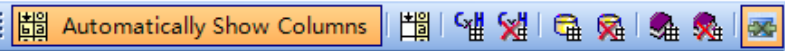

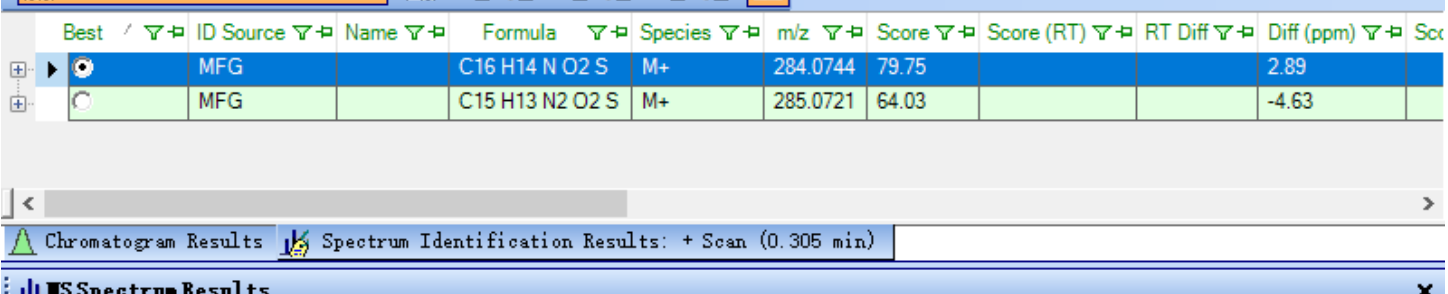

Illis Spectrun Results $x$

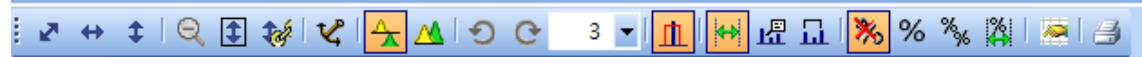

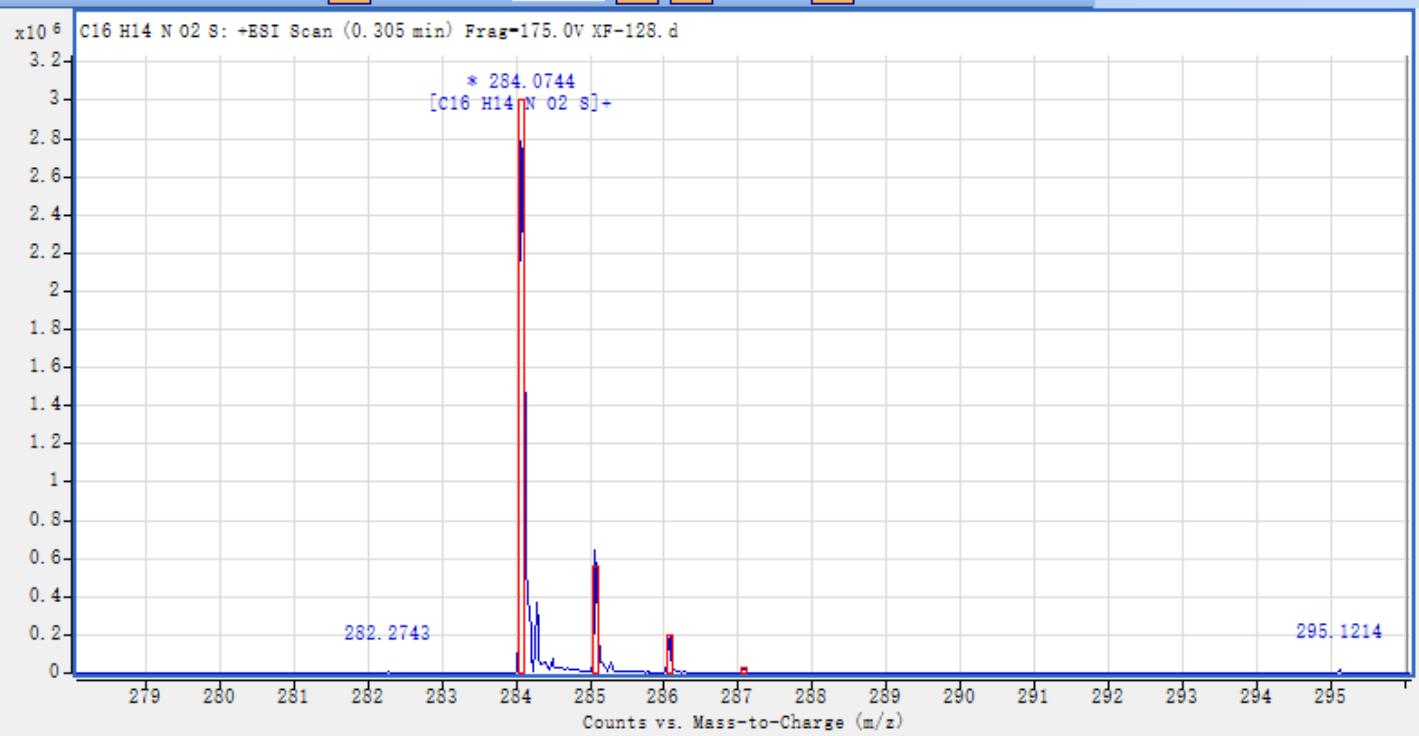

HPLC analysis of $\mathbf{5 f}$ 


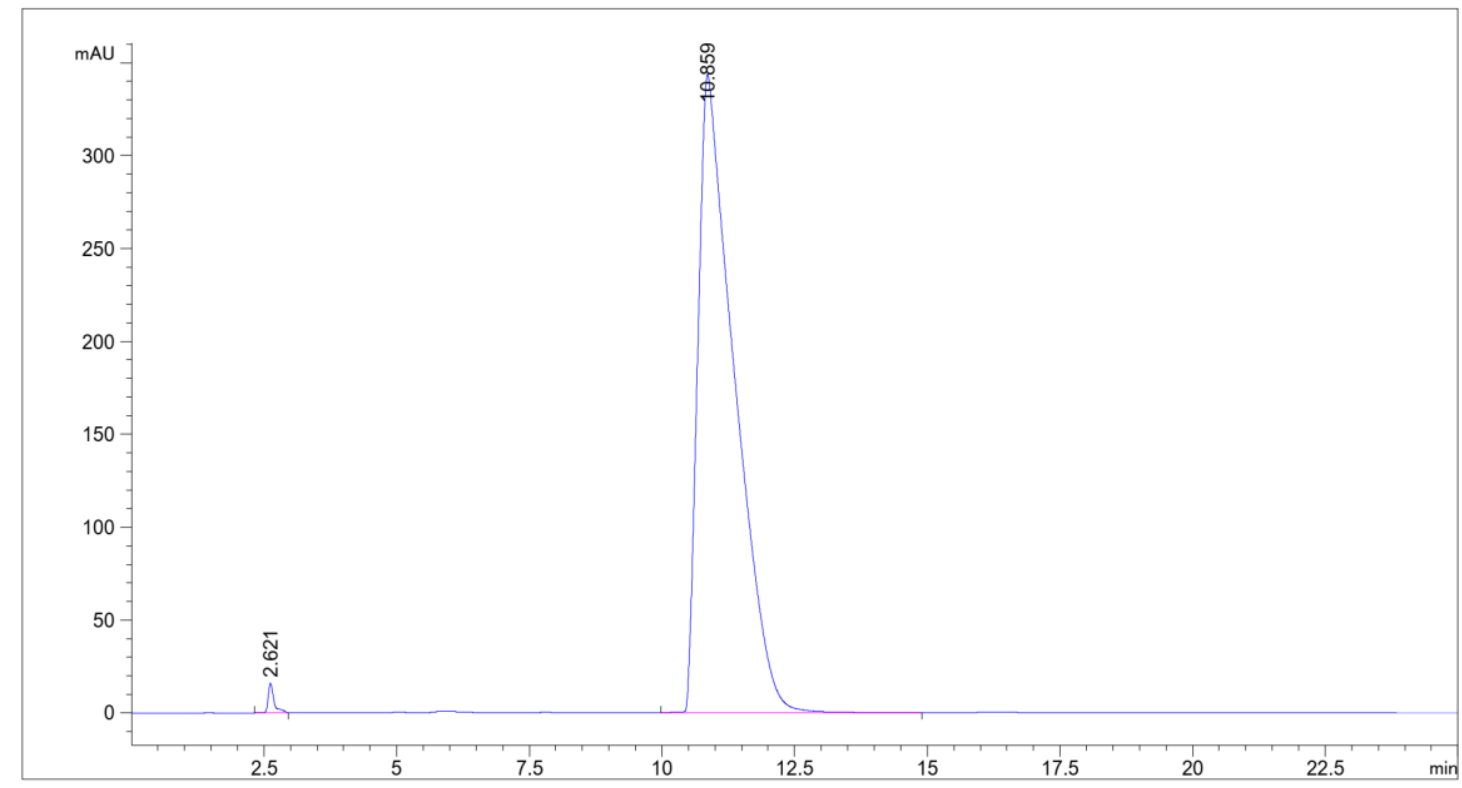

Area Percent Report

$\begin{array}{lll}\text { Sorted By } & : & \text { Signal } \\ \text { Multiplier } & : & 1.0000 \\ \text { Dilution } & : & 1.0000\end{array}$

Use Multiplier \& Dilution Factor with ISTDs

Signal 1: VWD1 A, Wavelength=254 nm

\begin{tabular}{|c|c|c|c|c|c|c|}
\hline $\begin{array}{c}\text { Peak } \\
\quad \#\end{array}$ & $\begin{array}{c}\text { RetTime } \\
\text { [min] }\end{array}$ & Type & $\begin{array}{l}\text { Width } \\
\text { [min] }\end{array}$ & $\begin{array}{c}\text { Area } \\
{\left[\mathrm{mAU}^{\star} \mathrm{s}\right]}\end{array}$ & $\begin{array}{l}\text { Height } \\
\text { [mAU] }\end{array}$ & $\begin{array}{c}\text { Area } \\
\frac{\circ}{0}\end{array}$ \\
\hline---1 & $|-------|$ & ---1 & ------- & $----------\mid$ & $----------\mid$ & $--------\mid$ \\
\hline 1 & 2.621 & $\mathrm{BB}$ & 0.1141 & 119.83575 & 15.86792 & 0.7276 \\
\hline 2 & 10.859 & BB & 0.6656 & $1.63497 \mathrm{e} 4$ & 343.34421 & 99.2724 \\
\hline
\end{tabular}

Totals : $\quad 1.64696 \mathrm{e} 4 \quad 359.21213$

${ }^{1} \mathrm{H}$ NMR spectrum of compound $\mathbf{5 g}$ 

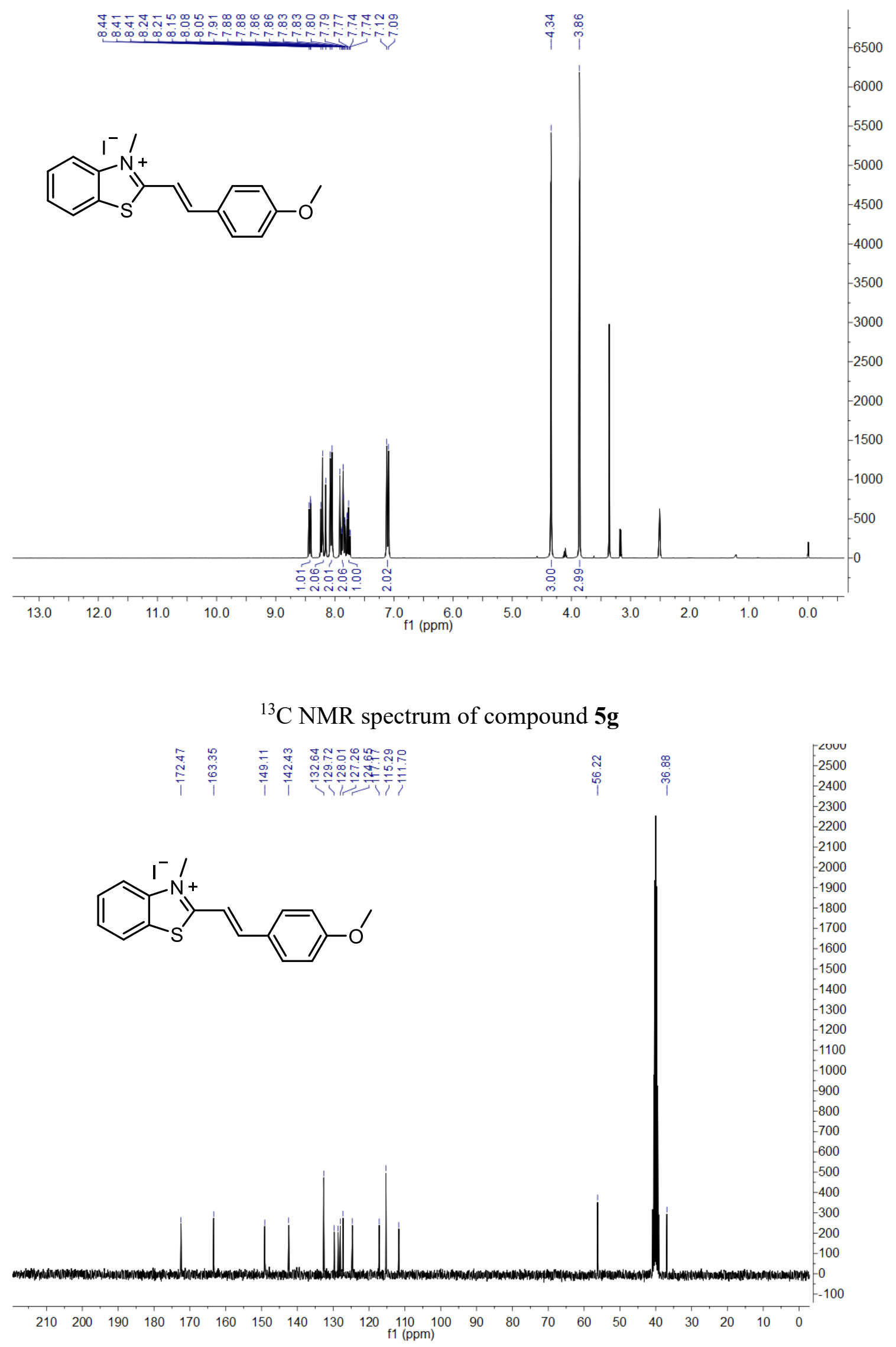
HRMS of compound $\mathbf{5 g}$

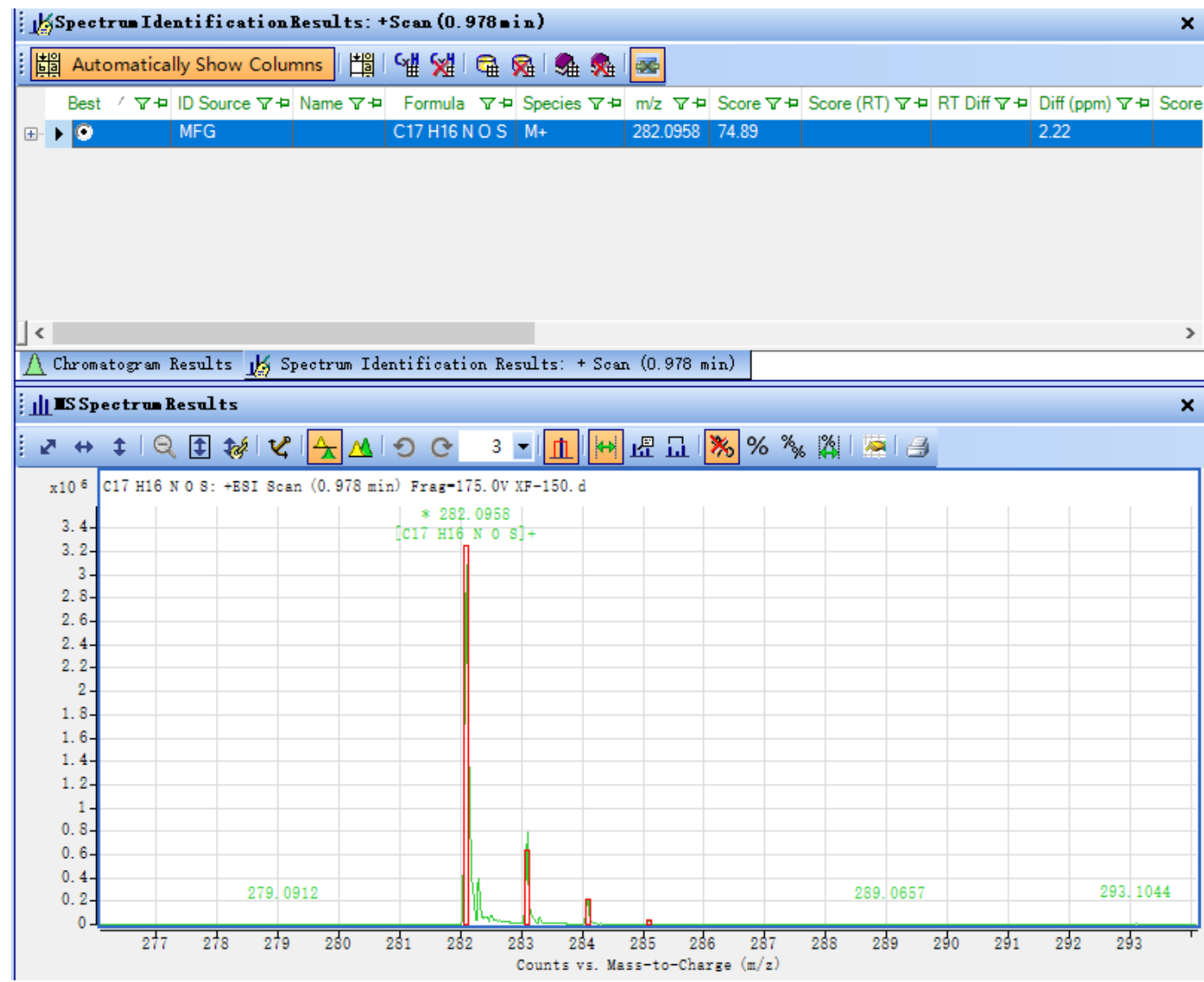




\section{HPLC analysis of $\mathbf{5 g}$}

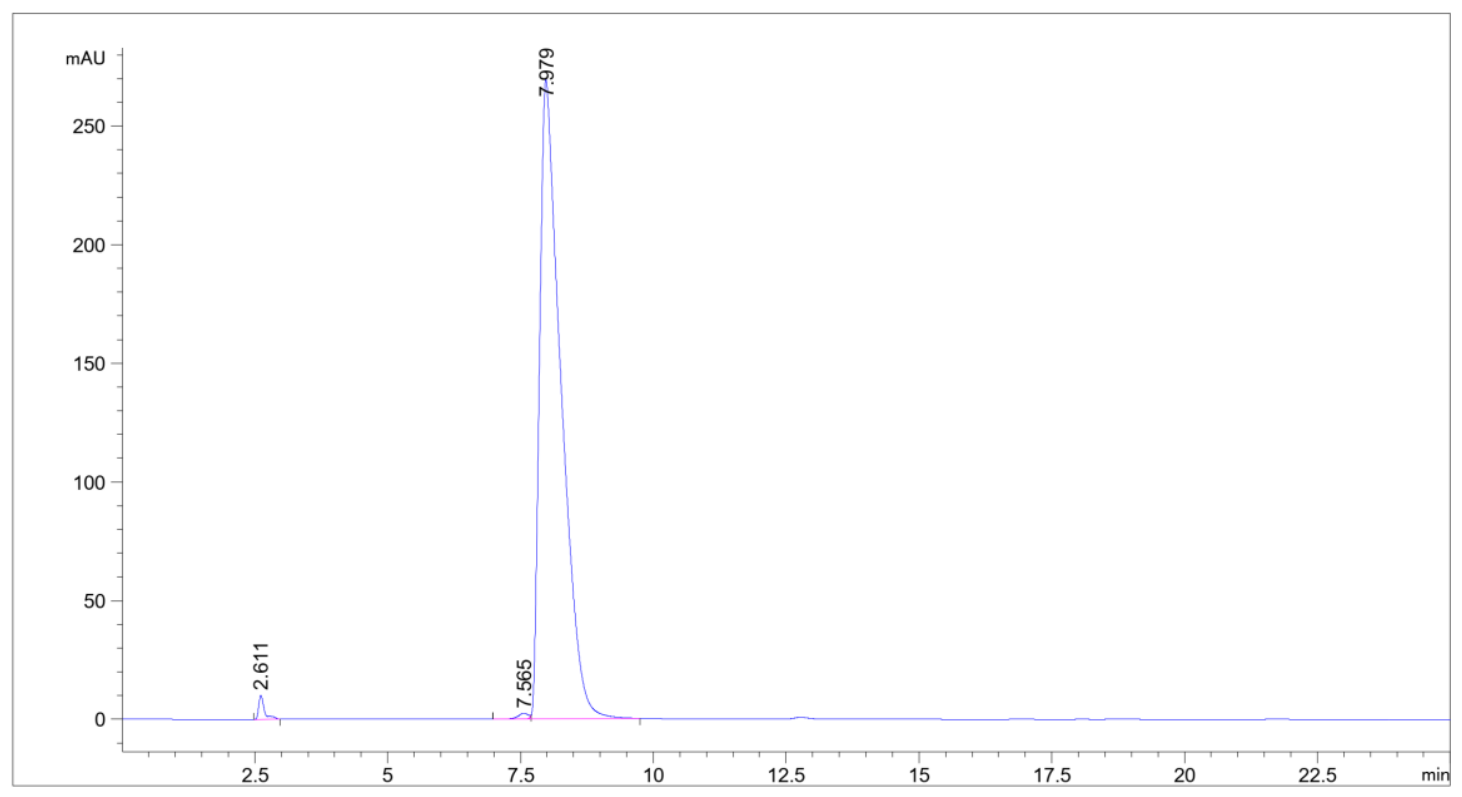

Area Percent Report

\begin{tabular}{|c|c|c|}
\hline Sorted By & : & Signal \\
\hline Multiplier & $:$ & 1.0000 \\
\hline Dilution & : & 1.0000 \\
\hline
\end{tabular}

Use Multiplier \& Dilution Factor with ISTDs

Signal 1: VWD1 A, Wavelength=254 nm

\begin{tabular}{|c|c|c|c|c|c|c|}
\hline $\begin{array}{c}\text { Peak } \\
\#\end{array}$ & $\begin{array}{c}\text { RetTime } \\
\text { [min] }\end{array}$ & Type & $\begin{array}{l}\text { Width } \\
\text { [min] }\end{array}$ & $\begin{array}{c}\text { Area } \\
{\left[\mathrm{mAU}{ }^{\star} \mathrm{S}\right]}\end{array}$ & $\begin{array}{l}\text { Height } \\
\text { [mAU] }\end{array}$ & $\begin{array}{c}\text { Area } \\
\frac{8}{\delta}\end{array}$ \\
\hline & 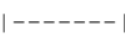 & & & --------- & -------- & $-------\mid$ \\
\hline 1 & 2.611 & BV $R$ & 0.1143 & 75.17915 & 10.04906 & 0.9844 \\
\hline 2 & 7.565 & BV $E$ & 0.2110 & 33.50993 & 2.41389 & 0.4388 \\
\hline 3 & 7.979 & VB $R$ & 0.4000 & 7528.72559 & 269.43283 & 98.5769 \\
\hline
\end{tabular}

Totals : $\quad 7637.41466 \quad 281.89578$ 


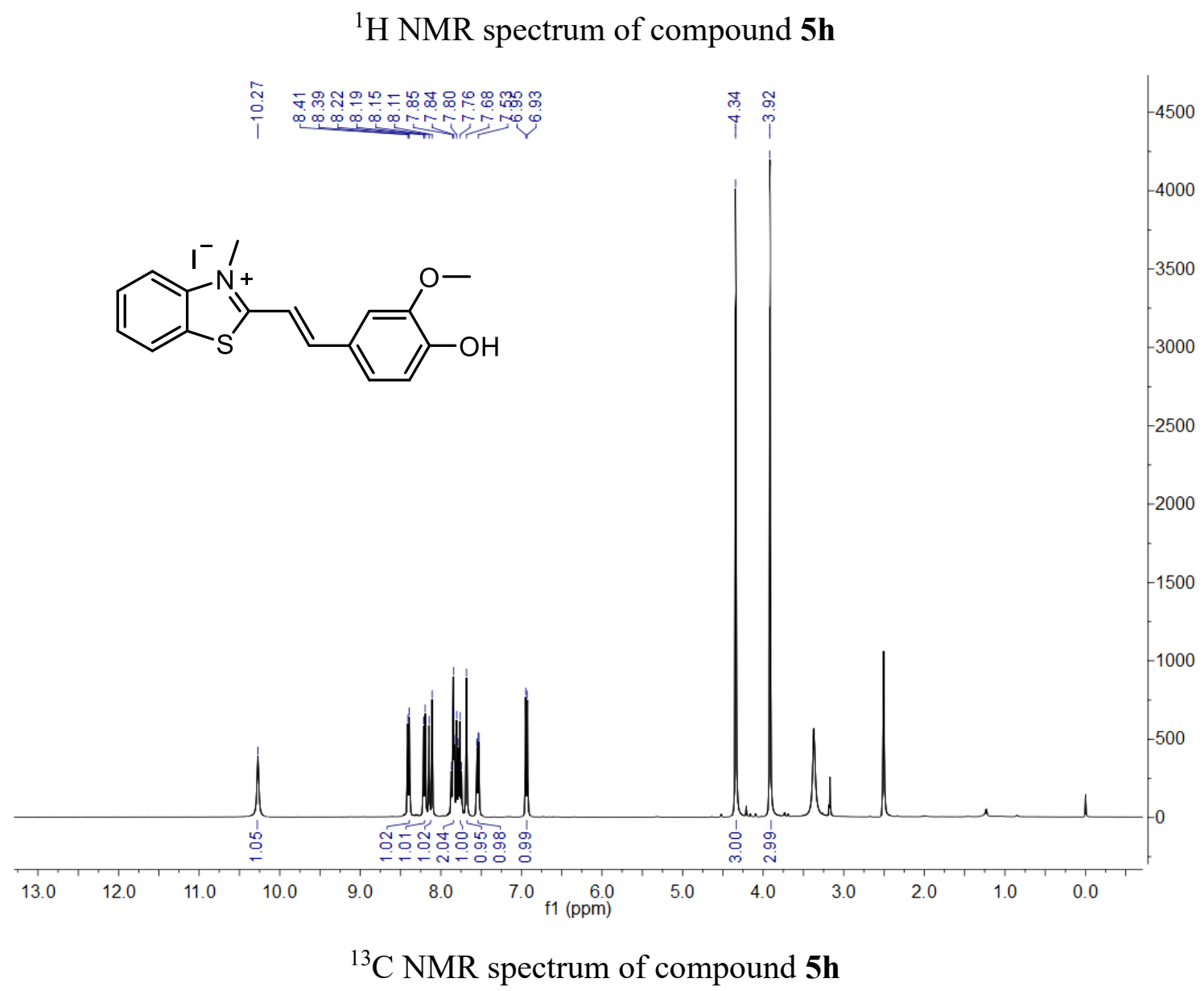




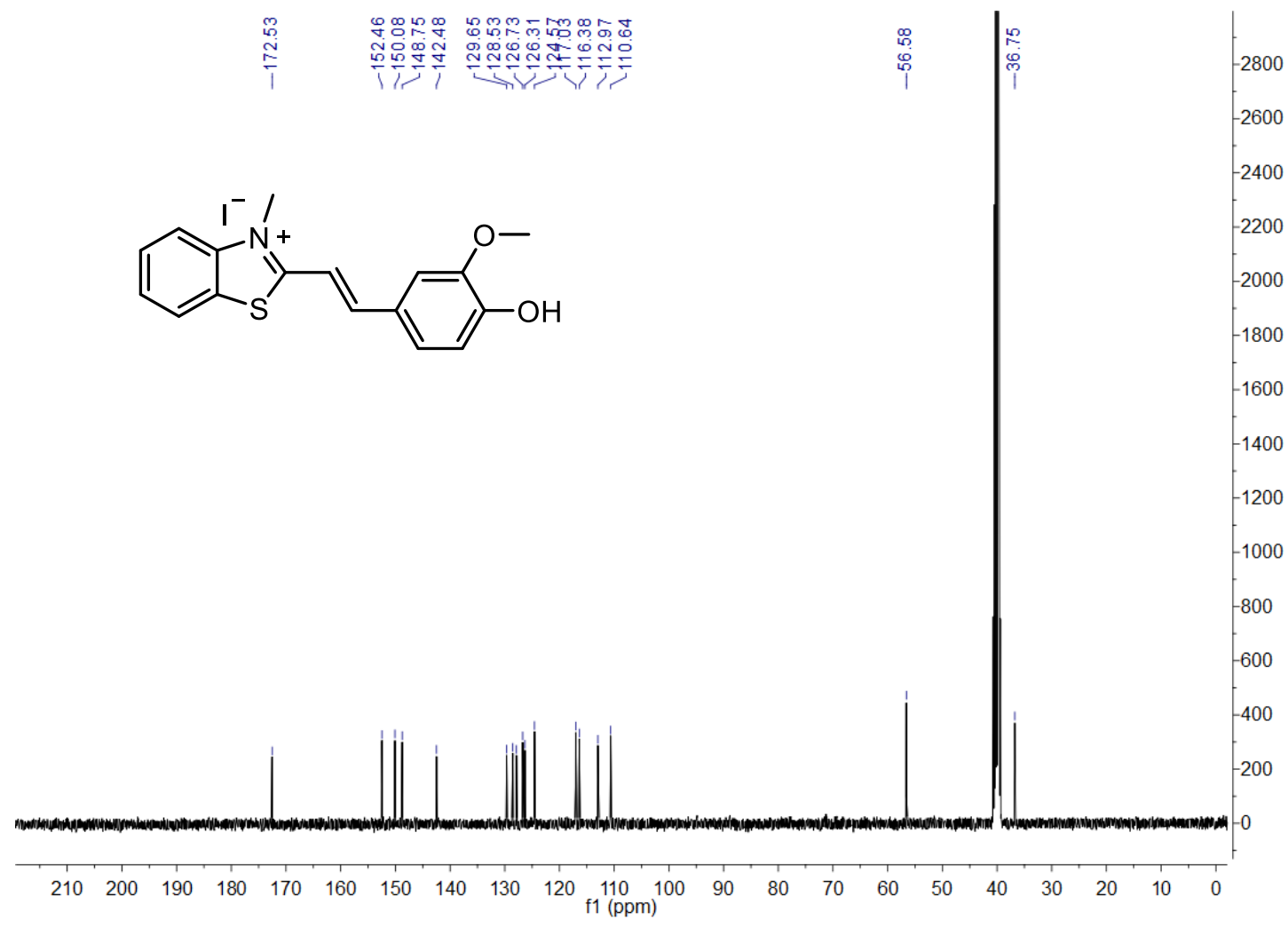

HRMS of compound $\mathbf{5 h}$

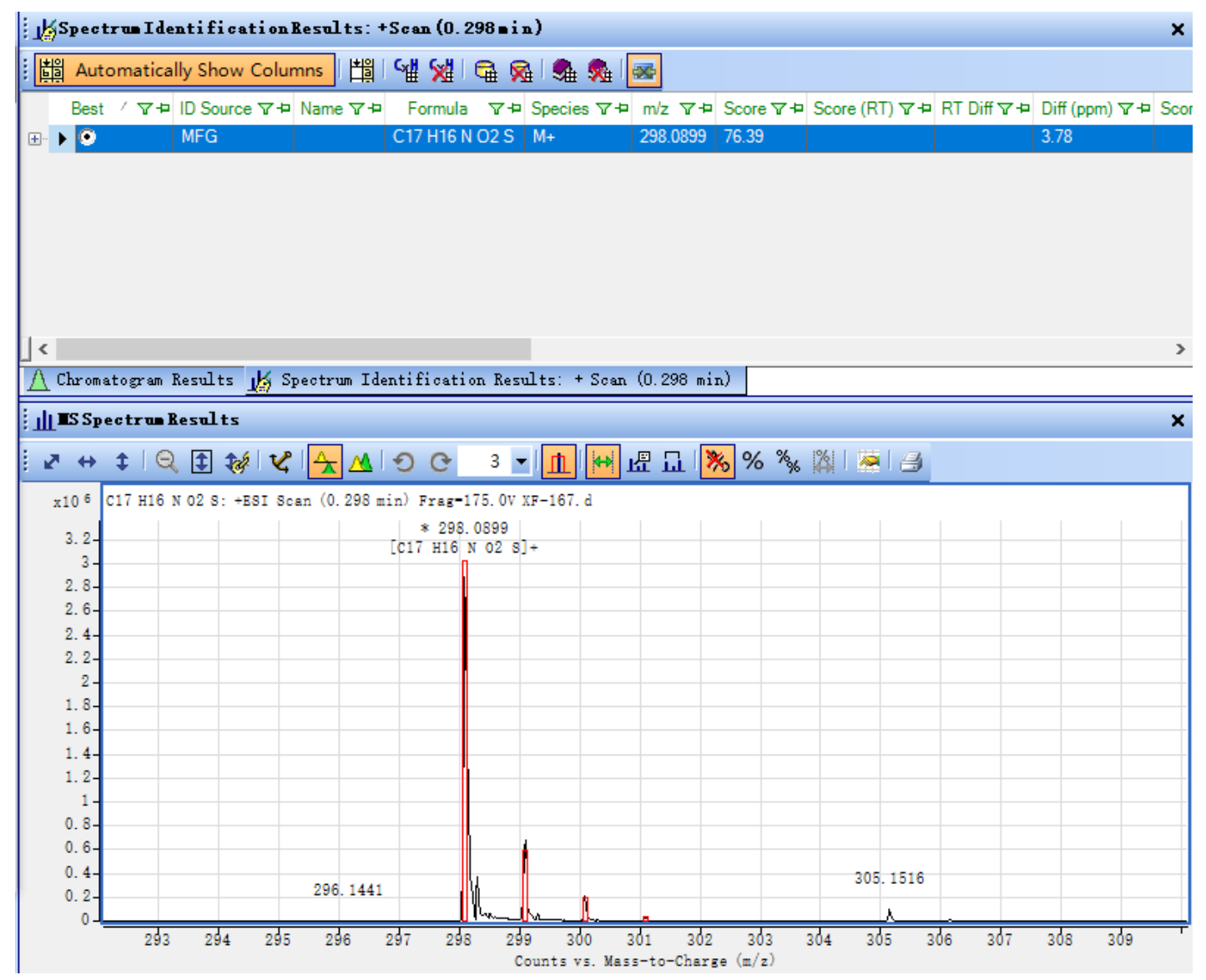


HPLC analysis of $\mathbf{5 h}$

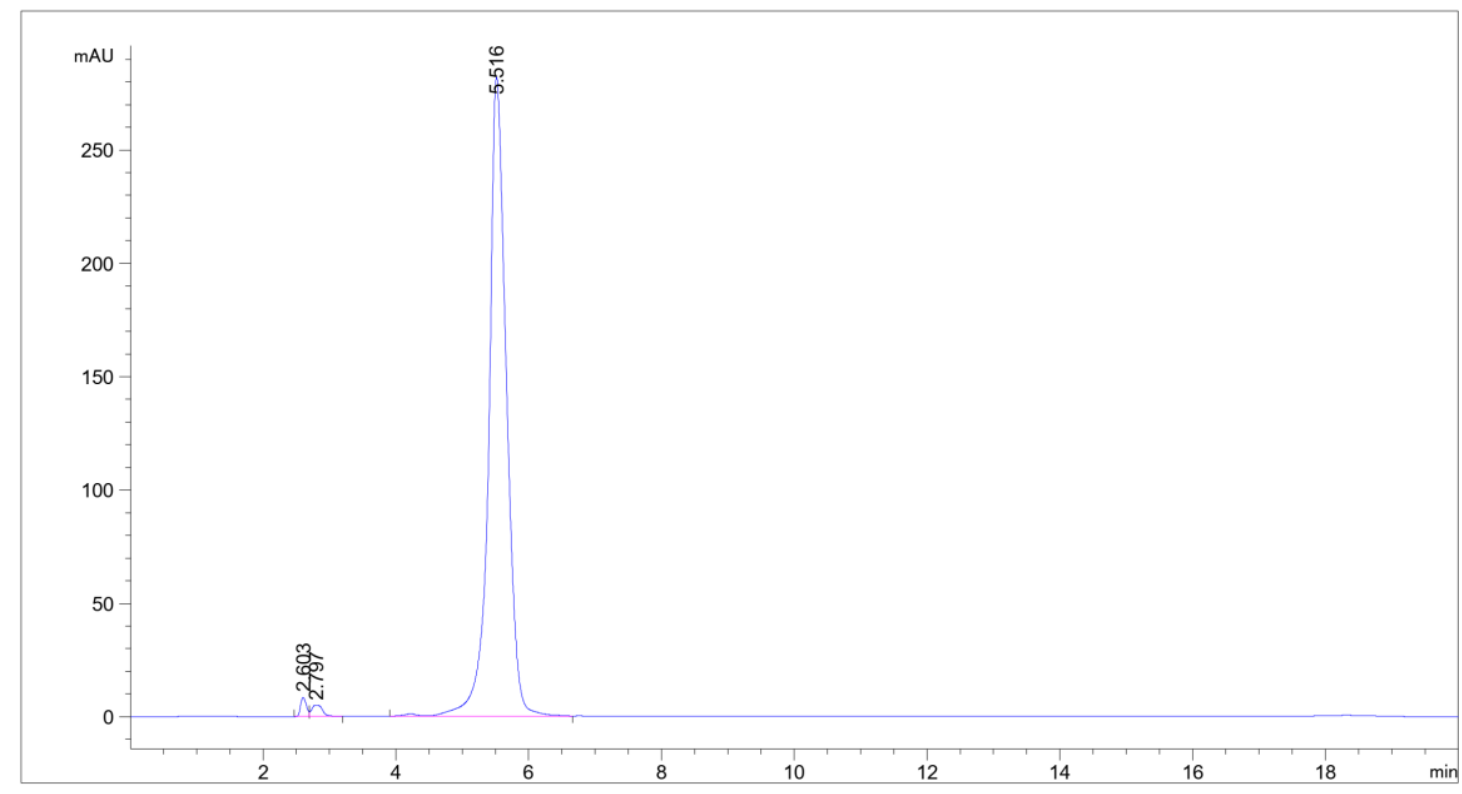

Area Percent Report

$\begin{array}{lll}\text { Sorted By } & : & \text { Signal } \\ \text { Multiplier } & : & 1.0000 \\ \text { Dilution } & : & 1.0000\end{array}$

Use Multiplier \& Dilution Factor with ISTDs

Signal 1: VWD1 A, Wavelength=254 nm

\begin{tabular}{|c|c|c|c|c|c|c|}
\hline $\begin{array}{c}\text { Peak } \\
\#\end{array}$ & $\begin{array}{c}\text { RetTime } \\
\text { [min] }\end{array}$ & Type & $\begin{array}{l}\text { Width } \\
\text { [min] }\end{array}$ & $\begin{array}{c}\text { Area } \\
{\left[\mathrm{mAU}^{*} \mathrm{~S}\right]}\end{array}$ & $\begin{array}{l}\text { Height } \\
{[\mathrm{mAU}]}\end{array}$ & $\begin{array}{c}\text { Area } \\
8\end{array}$ \\
\hline \multicolumn{7}{|c|}{ 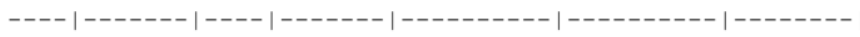 } \\
\hline 1 & 2.603 & BV & 0.0979 & 52.05815 & 8.44206 & 0.9809 \\
\hline 2 & 2.797 & VB & 0.1800 & 56.19205 & 4.93005 & 1.0588 \\
\hline 3 & 5.516 & VB $R$ & 0.2722 & 5199.00781 & 281.81021 & 97.9603 \\
\hline & s : & & & 5307.25801 & 295.18233 & \\
\hline
\end{tabular}


${ }^{1} \mathrm{H}$ NMR spectrum of compound $\mathbf{5 i}$

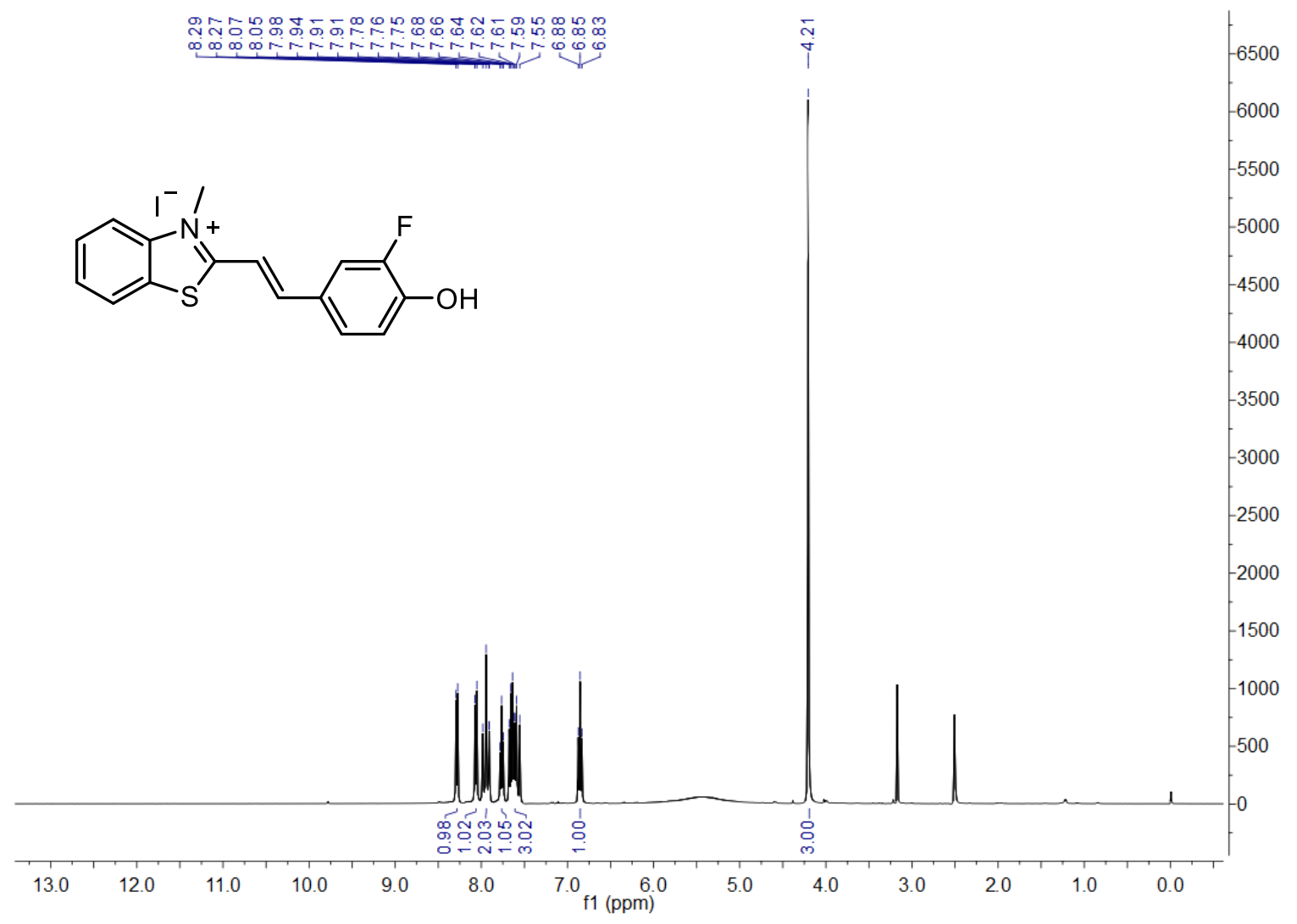

${ }^{13} \mathrm{C}$ NMR spectrum of compound $\mathbf{5 i}$ 


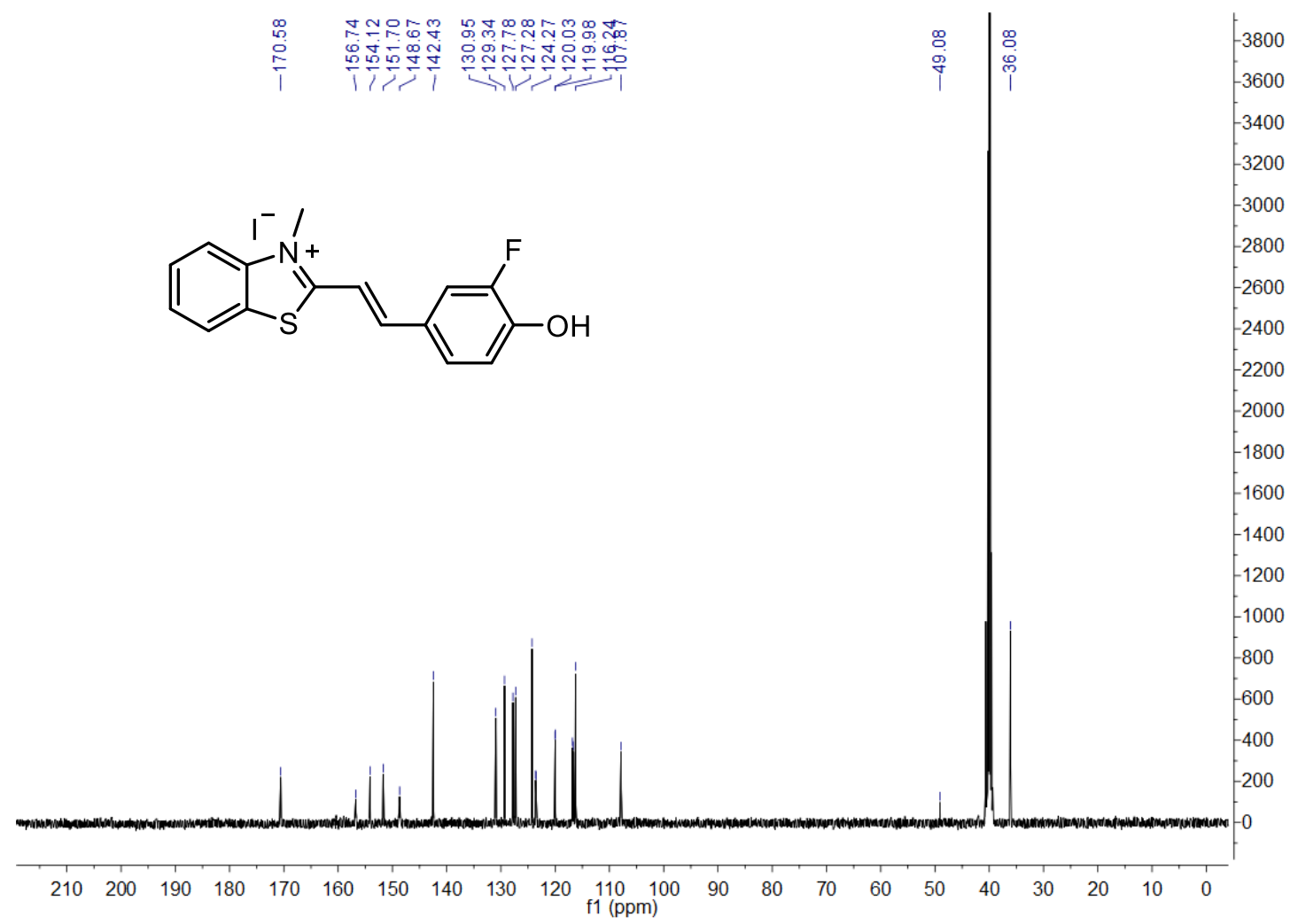

HRMS of compound $\mathbf{5 i}$

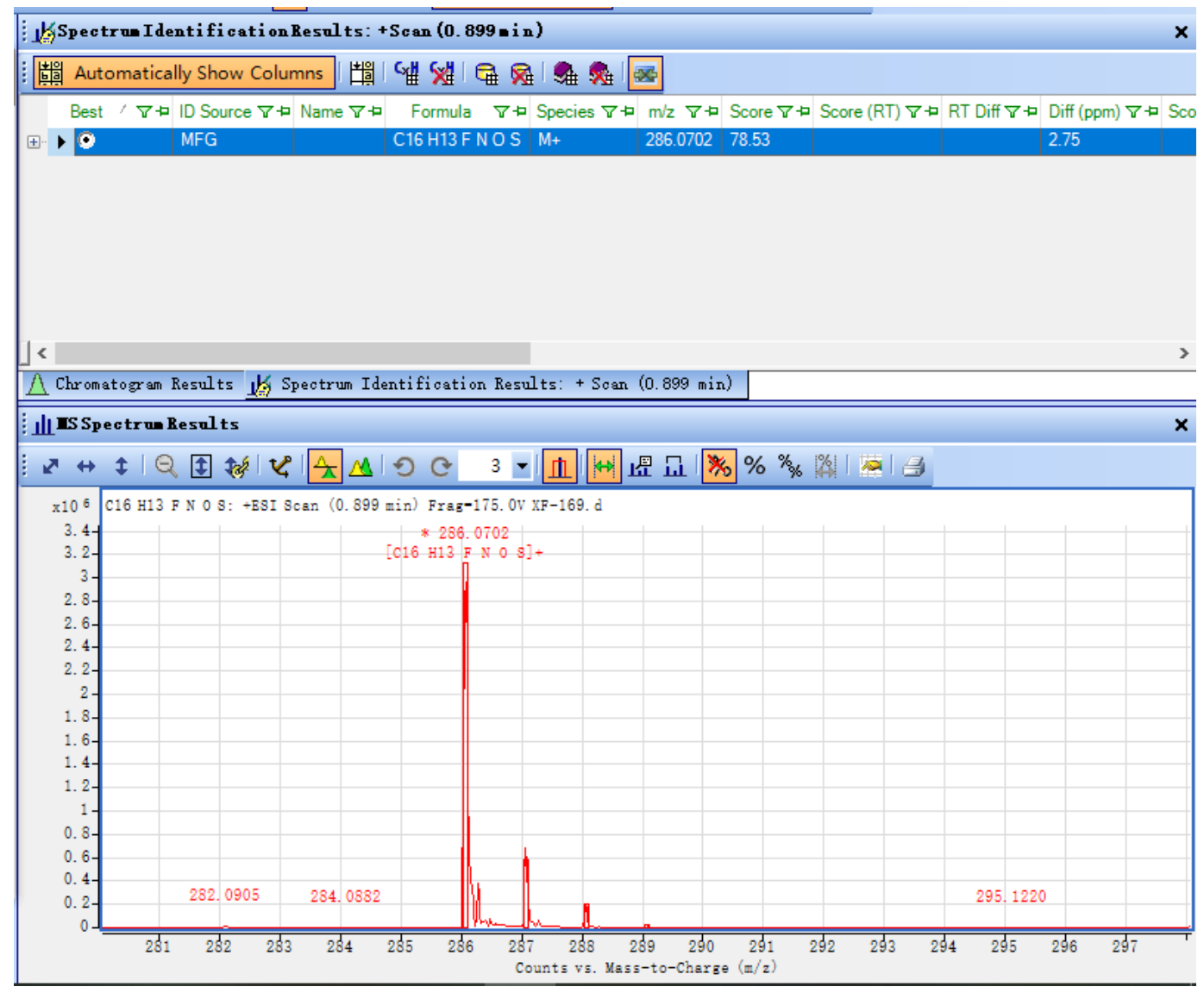




\section{HPLC analysis of $\mathbf{5 i}$}

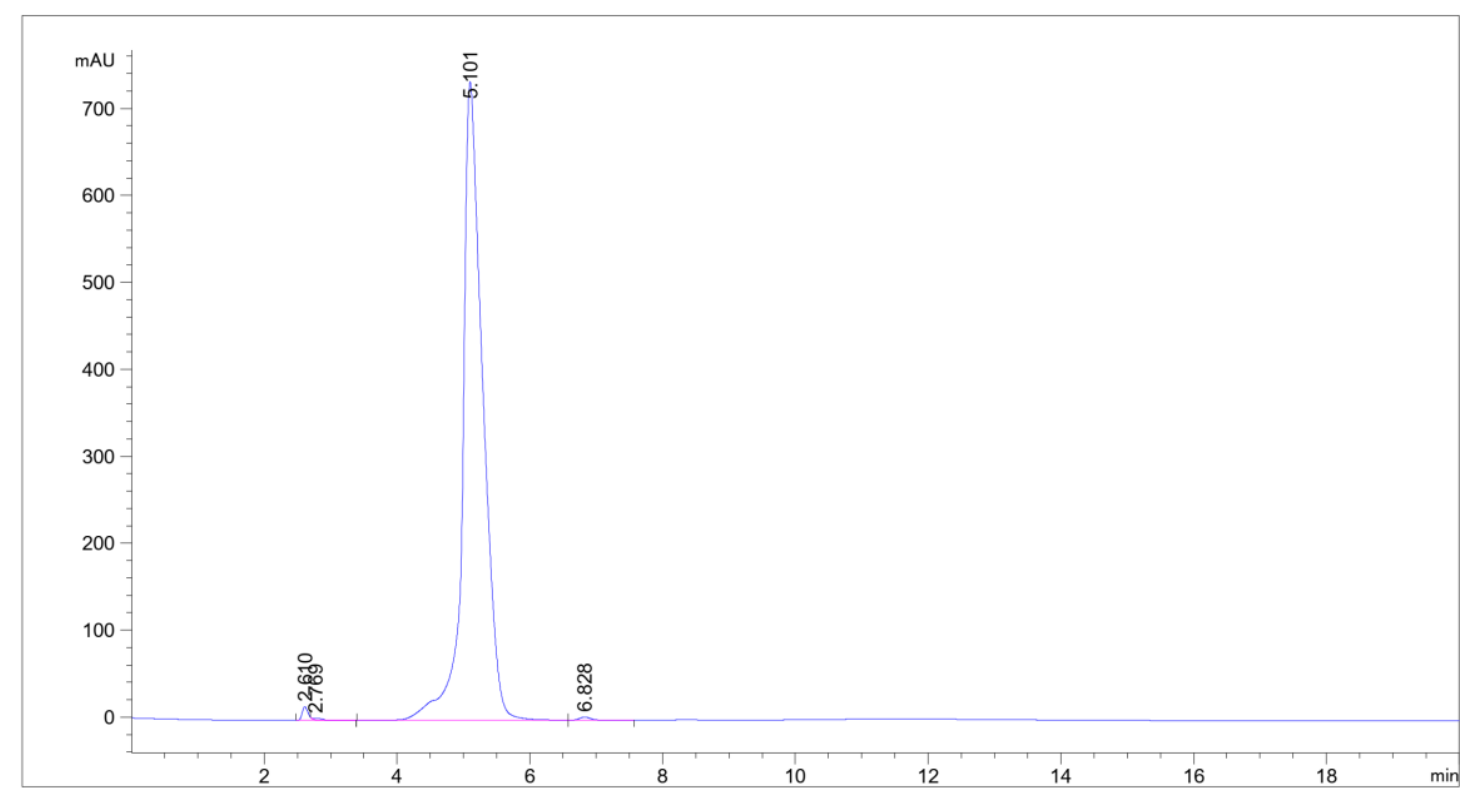

Area Percent Report

$\begin{array}{lll}\text { Sorted By } & : & \text { Signal } \\ \text { Multiplier } & : & 1.0000 \\ \text { Dilution } & : & 1.0000\end{array}$

Use Multiplier \& Dilution Factor with ISTDs

Signal 1: VWD1 A, Wavelength=254 nm

\begin{tabular}{|c|c|c|c|c|c|c|}
\hline $\begin{array}{c}\text { Peak } \\
\#\end{array}$ & $\begin{array}{c}\text { RetTime } \\
\text { [min] }\end{array}$ & Type & $\begin{array}{l}\text { Width } \\
\text { [min] }\end{array}$ & $\begin{array}{c}\text { Area } \\
{[\mathrm{mAU} * \mathrm{~s}]}\end{array}$ & $\begin{array}{l}\text { Height } \\
\text { [mAU] }\end{array}$ & $\begin{array}{c}\text { Area } \\
\frac{\circ}{\delta}\end{array}$ \\
\hline & & & ----- & ---------- & $-------\mid$ & $------\mid$ \\
\hline 1 & 2.610 & BV $R$ & 0.1003 & 101.23065 & 15.69326 & 0.6453 \\
\hline 2 & 2.769 & VB E & 0.1470 & 19.87670 & 1.73117 & 0.1267 \\
\hline 3 & 5.101 & $\mathrm{BB}$ & 0.3026 & $1.55216 \mathrm{e} 4$ & 734.44177 & 98.9444 \\
\hline 4 & 6.828 & $\mathrm{BB}$ & 0.1833 & 44.48724 & 3.67244 & 0.2836 \\
\hline
\end{tabular}

${ }^{1} \mathrm{H}$ NMR spectrum of compound $\mathbf{5 j}$ 


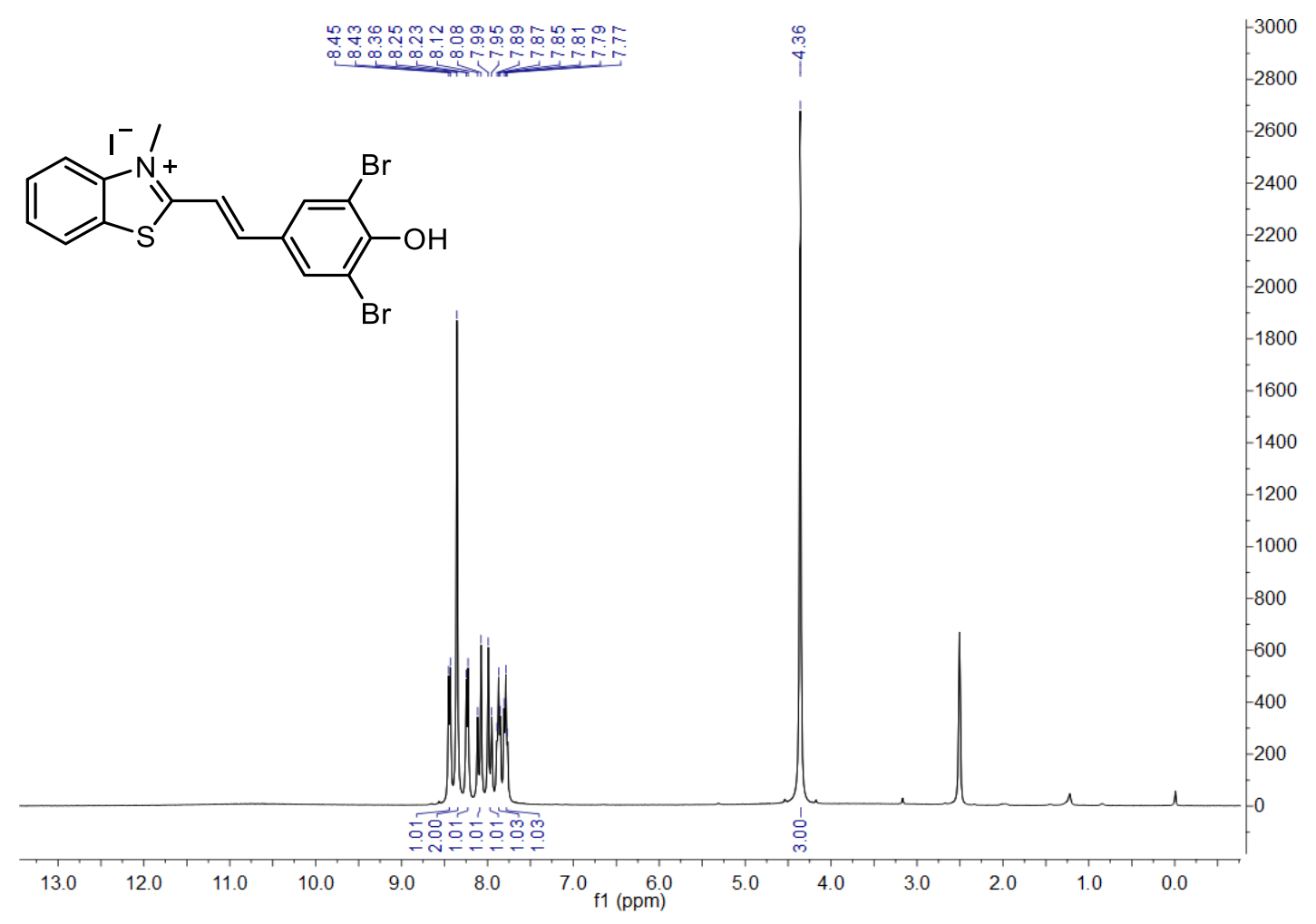

${ }^{13} \mathrm{C}$ NMR spectrum of compound $\mathbf{5} \mathbf{j}$

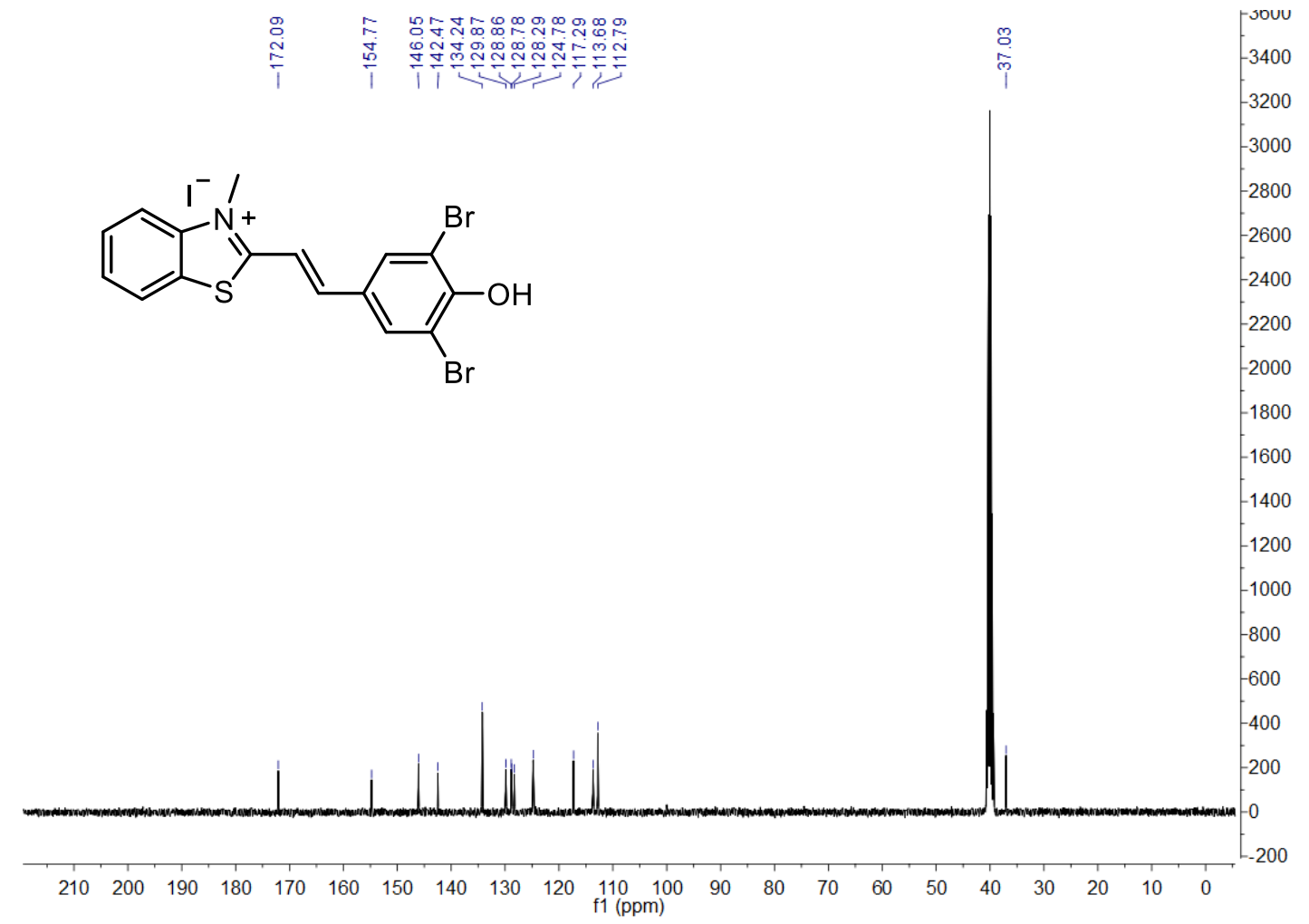

HRMS of compound $\mathbf{5 j}$ 
Spectrum from 20200602.wiff (sample 66) - 159, Experiment 1, +TOF MS (50 - 1000) from 0.179 to $0.227 \mathrm{~min}$

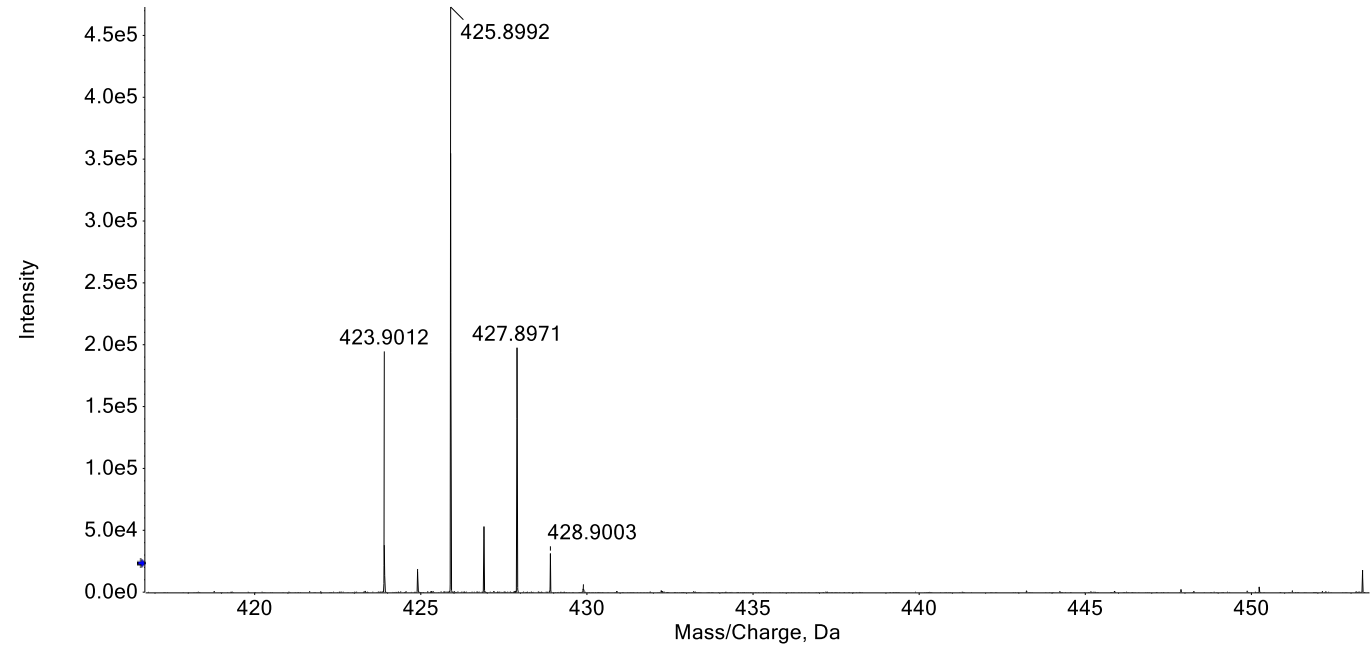

Hit Formula $\quad \mathrm{m} / \mathrm{z} \quad$ RDB $\mathrm{ppm}$ MS Rank MSMS ppm MSMS Rank Found

\begin{tabular}{l|l|l|l|l|l|l|}
\hline 1 & $\mathrm{C}_{16} \mathrm{H}_{12} \mathrm{NOSBr}_{2}{ }^{+}$ & 423.9001 & 11.0 & 2.4 & 1 & NA/NA
\end{tabular}

\section{HPLC analysis of $\mathbf{5 j}$}

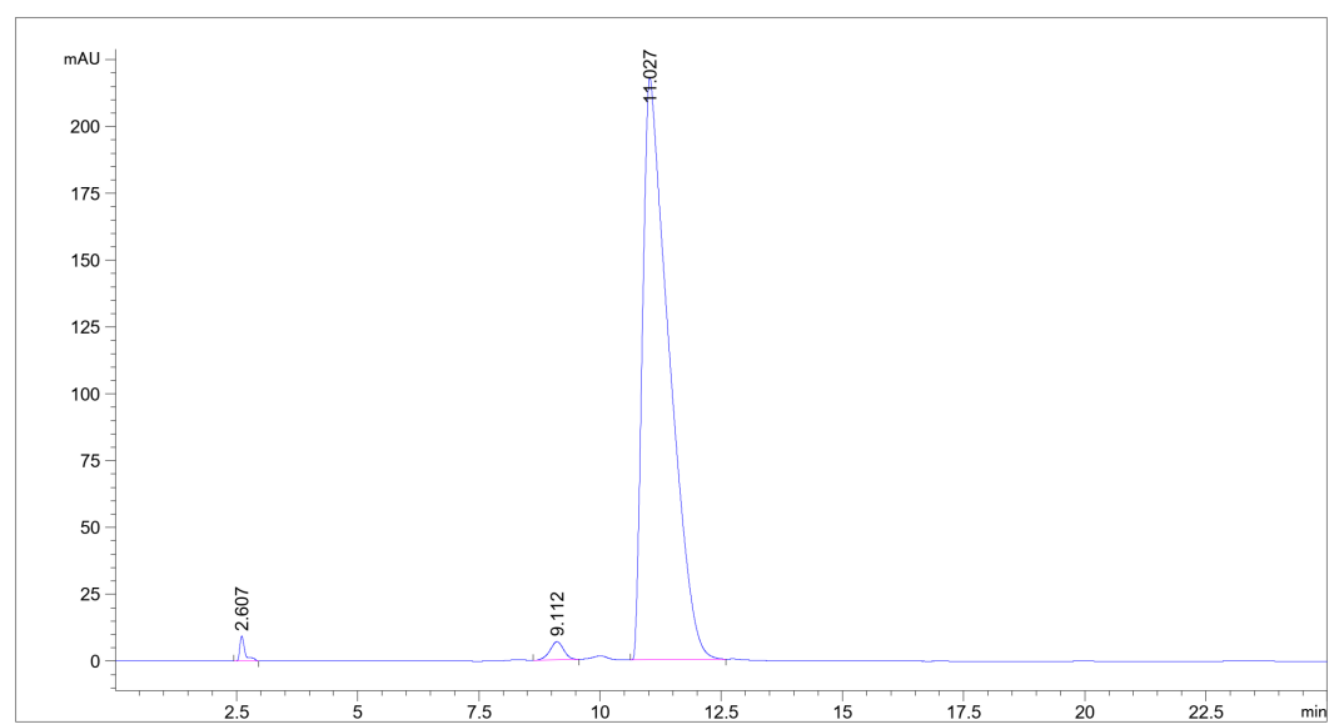

Area Percent Report

$\begin{array}{lll}\text { Sorted By } & : & \text { Signal } \\ \text { Multiplier } & : & 1.0000 \\ \text { Dilution } & : & 1.0000\end{array}$

Use Multiplier \& Dilution Factor with ISTDs

Signal 1: VWD1 A, Wavelength $=254 \mathrm{~nm}$

\begin{tabular}{|c|c|c|c|c|c|c|}
\hline $\begin{array}{c}\text { Peak } \\
\quad \#\end{array}$ & $\begin{array}{c}\text { RetTime } \\
\text { [min] }\end{array}$ & Type & $\begin{array}{l}\text { Width } \\
\text { [min] }\end{array}$ & $\begin{array}{c}\text { Area } \\
{\left[\mathrm{mAU}^{*} \mathrm{~s}\right]}\end{array}$ & $\begin{array}{l}\text { Height } \\
\text { [mAU] }\end{array}$ & $\begin{array}{c}\text { Area } \\
\frac{\circ}{\partial}\end{array}$ \\
\hline-1 & 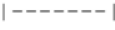 & $--\mid$ & ----- & 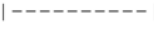 & 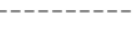 & ----- \\
\hline 1 & 2.607 & BV $R$ & 0.1131 & 69.96418 & 9.26225 & 0.8291 \\
\hline 2 & 9.112 & $\mathrm{BB}$ & 0.3027 & 135.22519 & 6.80932 & 1.6025 \\
\hline 3 & 11.027 & BB & 0.5365 & 8233.39844 & 217.56618 & 97.5684 \\
\hline & & & & 8438.58781 & 233.63775 & \\
\hline
\end{tabular}



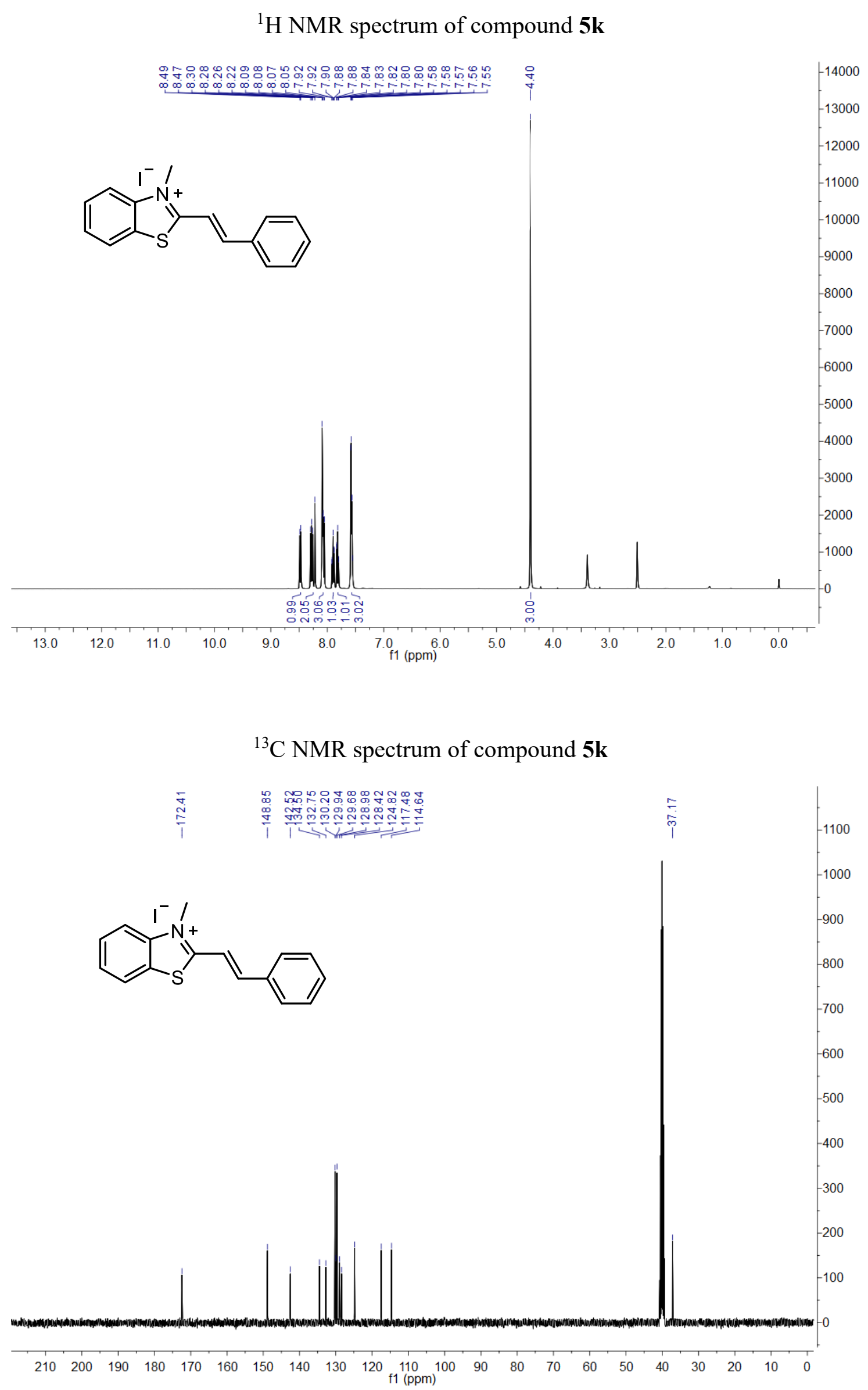


\section{HRMS of compound $\mathbf{5 k}$}

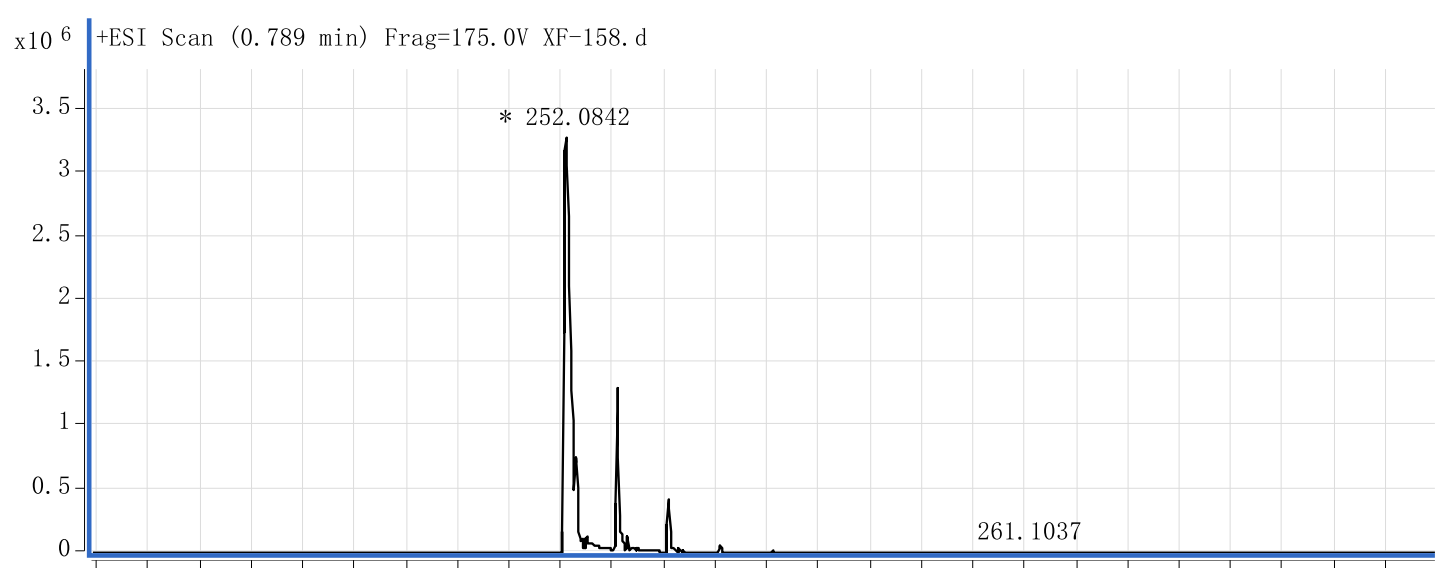

244245246247248249250251252253254255256257258259260261262263264265266267268 Counts vs. Mass-to-Charge $(\mathrm{m} / \mathrm{z})$

\begin{tabular}{|l|l|l|l|l|l|l|l|l|l|}
\hline Hit & Formula & Calc m/z & RDB & ppm & MS Rank & MSMS ppm & MSMS Rank & Found \\
\hline 1 & $\mathrm{C}_{16} \mathrm{H}_{14} \mathrm{NS}^{+}$ & 252.0841 & 11.0 & 0.4 & 1 & & & NA/NA \\
\hline
\end{tabular}

\section{HPLC analysis of $\mathbf{5 k}$}

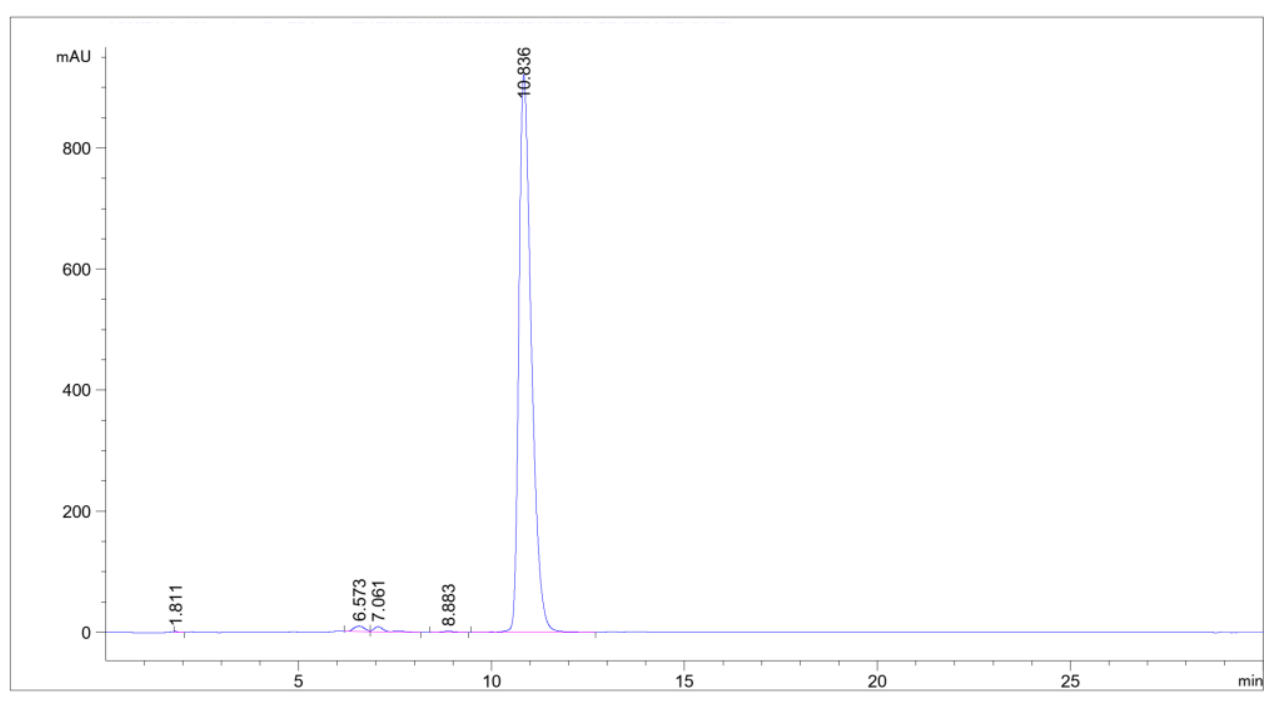

Area Percent Report

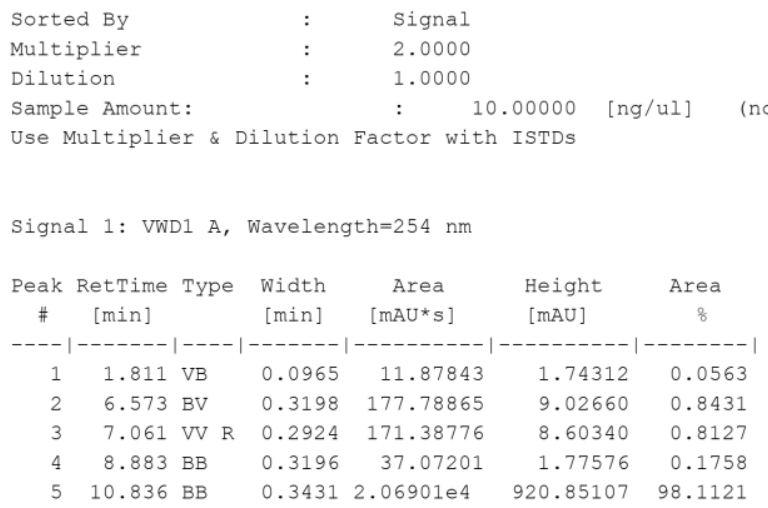



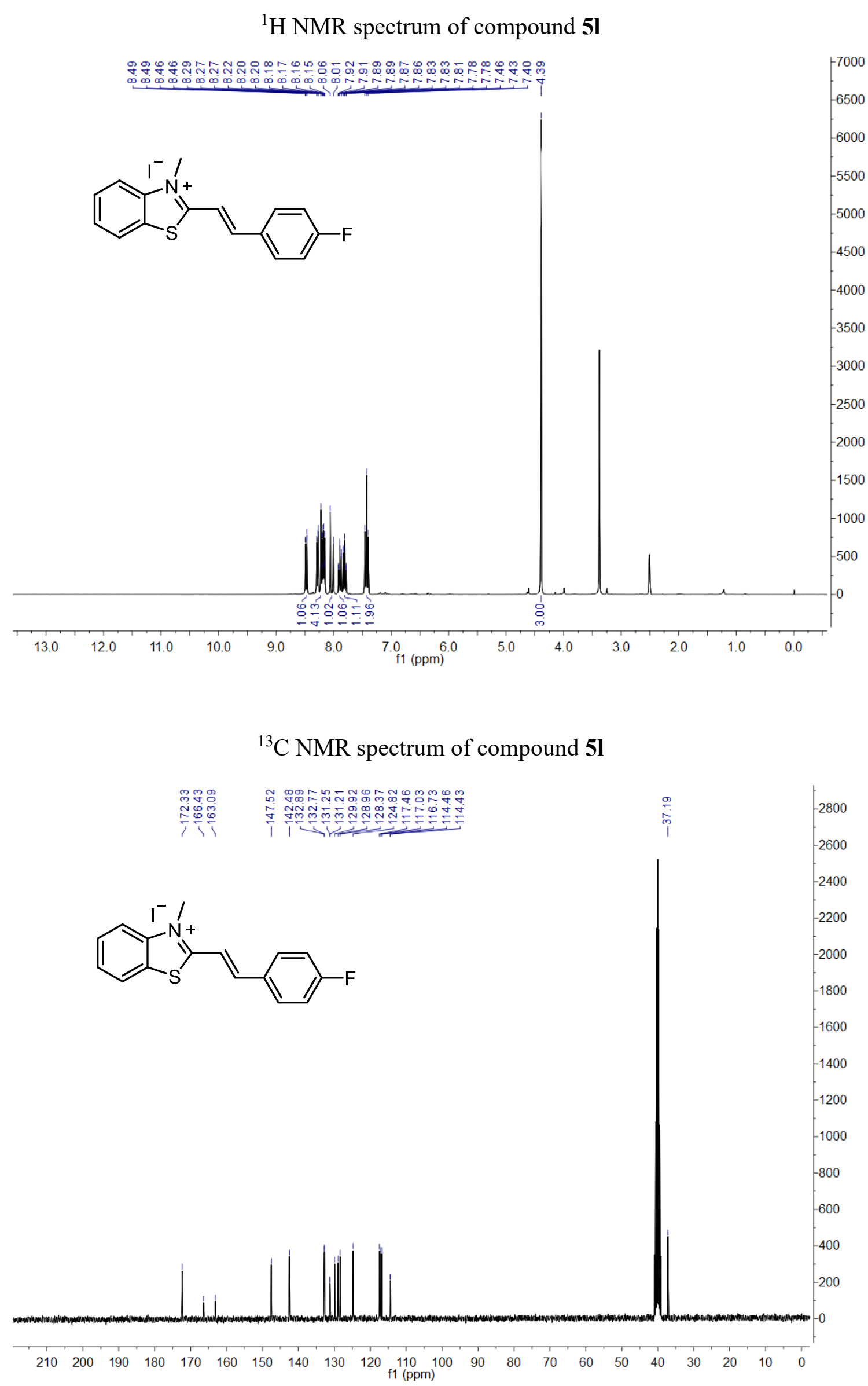
HRMS of compound $\mathbf{5 l}$

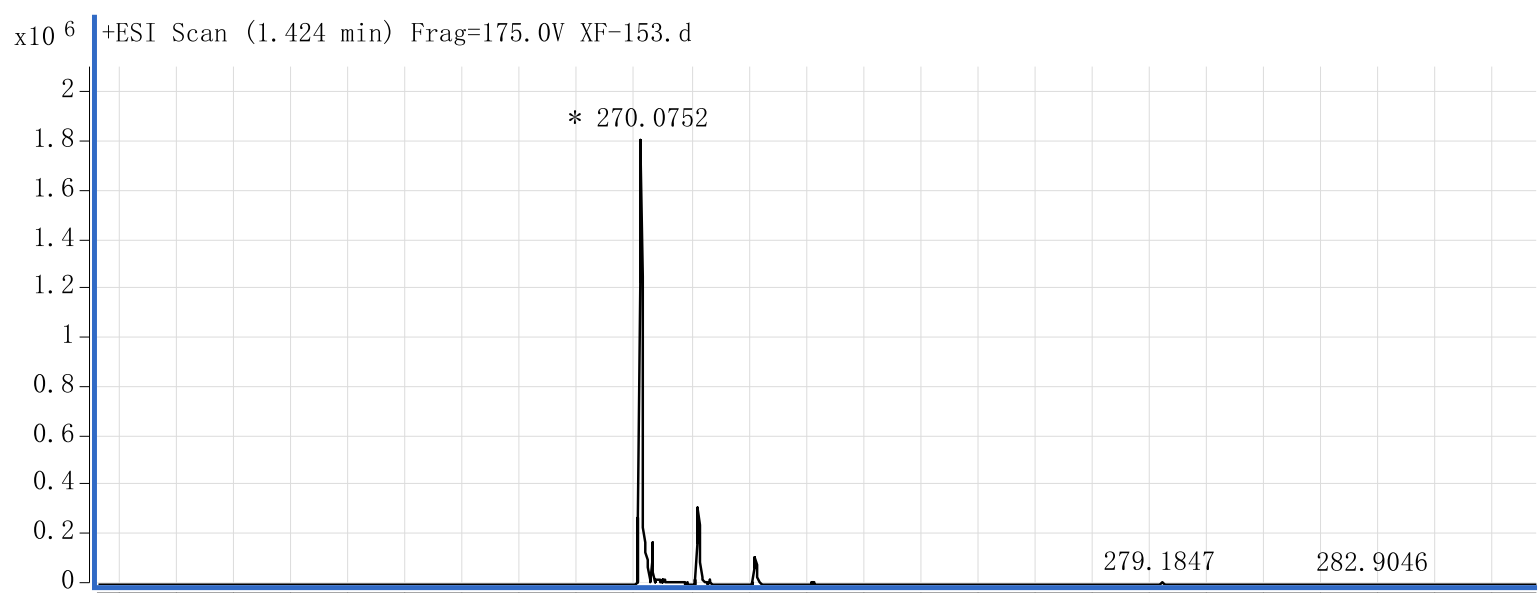

$\begin{array}{lllllllllllllllllllllllll}261 & 262 & 263 & 264 & 265 & 266 & 267 & 268 & 269 & 270 & 271 & 272 & 273 & 274 & 275 & 276 & 277 & 278 & 279 & 280 & 281 & 282 & 283 & 284 & 285\end{array}$ Counts vs. Mass-to-Charge $(\mathrm{m} / \mathrm{z})$

\begin{tabular}{|l|l|l|l|l|l|l|l|l|l|}
\hline Hit & Formula & Calc $\mathrm{m} / \mathrm{z}$ & RDB & ppm & MS Rank & MSMS ppm & MSMS Rank & Found \\
\hline 1 & $\mathrm{C}_{16} \mathrm{H}_{13} \mathrm{FNS}^{+}$ & 270.0747 & 11.0 & 1.8 & 1 & & & NA/NA \\
\hline
\end{tabular}

HPLC analysis of $\mathbf{5 l}$ 


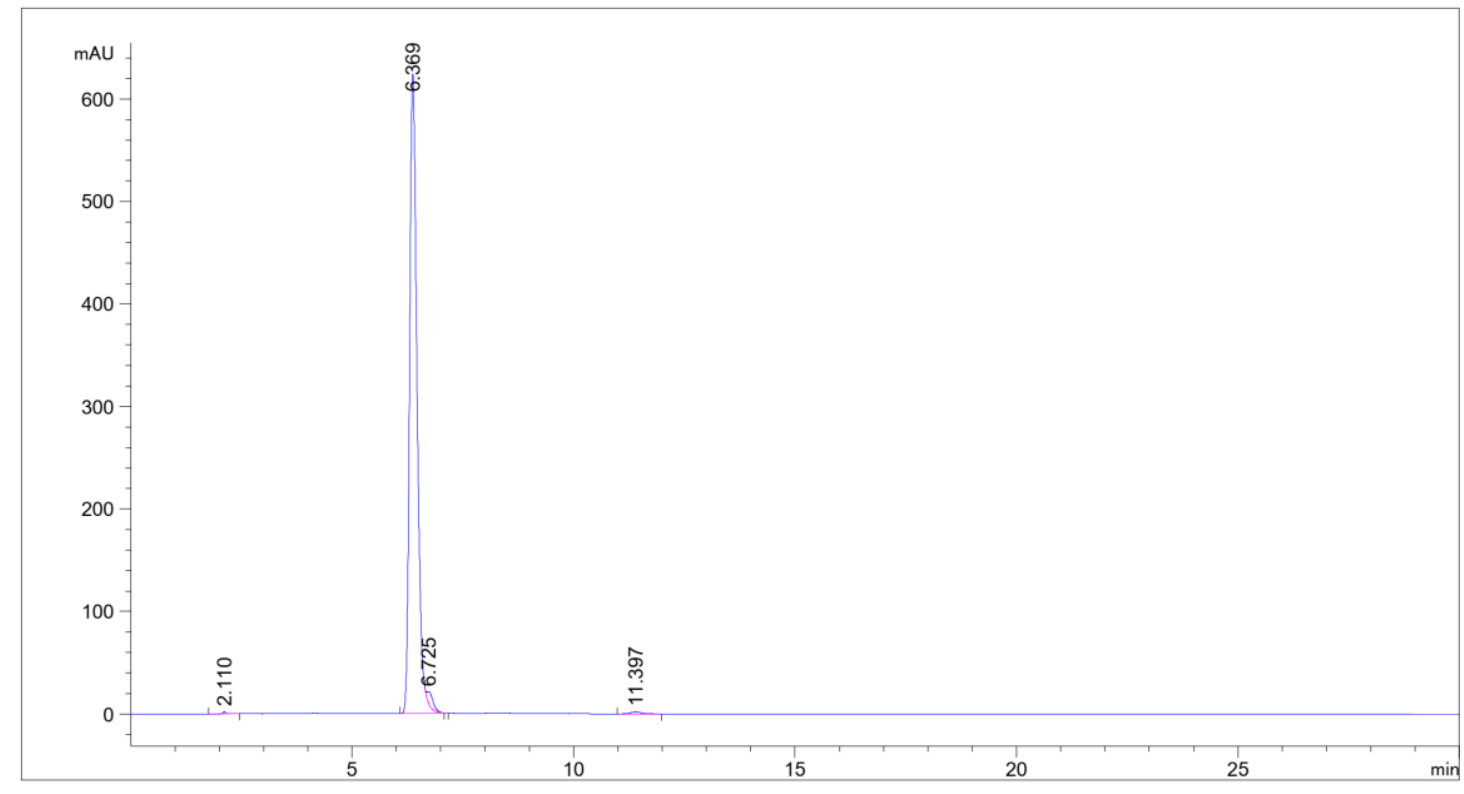

Area Percent Report

$\begin{array}{lll}\text { Sorted By } & : & \text { Signal } \\ \text { Multiplier } & : & 1.0000 \\ \text { Dilution } & : & 1.0000\end{array}$

Use Multiplier \& Dilution Factor with ISTDs

Signal 1: VWD1 A, Wavelength=254 nm

\begin{tabular}{|c|c|c|c|c|c|c|}
\hline $\begin{array}{c}\text { Peak } \\
\text { \# }\end{array}$ & $\begin{array}{c}\text { RetTime } \\
\text { [min] }\end{array}$ & Type & $\begin{array}{c}\text { Width } \\
\text { [min] }\end{array}$ & $\begin{array}{c}\text { Area } \\
{\left[\mathrm{mAU}{ }^{\star} \mathrm{s}\right]}\end{array}$ & $\begin{array}{l}\text { Height } \\
{[\mathrm{mAU}]}\end{array}$ & $\begin{array}{c}\text { Area } \\
\frac{\circ}{0}\end{array}$ \\
\hline & & & & & & \\
\hline 1 & 2.110 & BB & 0.0597 & 9.57940 & 2.34399 & 275 \\
\hline 2 & 6.369 & BV $R$ & 0.1804 & 7345.61133 & 623.66968 & 97.7474 \\
\hline 3 & 6.725 & VB E & 0.1668 & 128.29704 & 11.10671 & 1.7072 \\
\hline 4 & 11.397 & $\mathrm{BB}$ & 0.2657 & 31.40487 & 1.82596 & 0.4179 \\
\hline
\end{tabular}

Totals :

$7514.89264 \quad 638.94633$ 

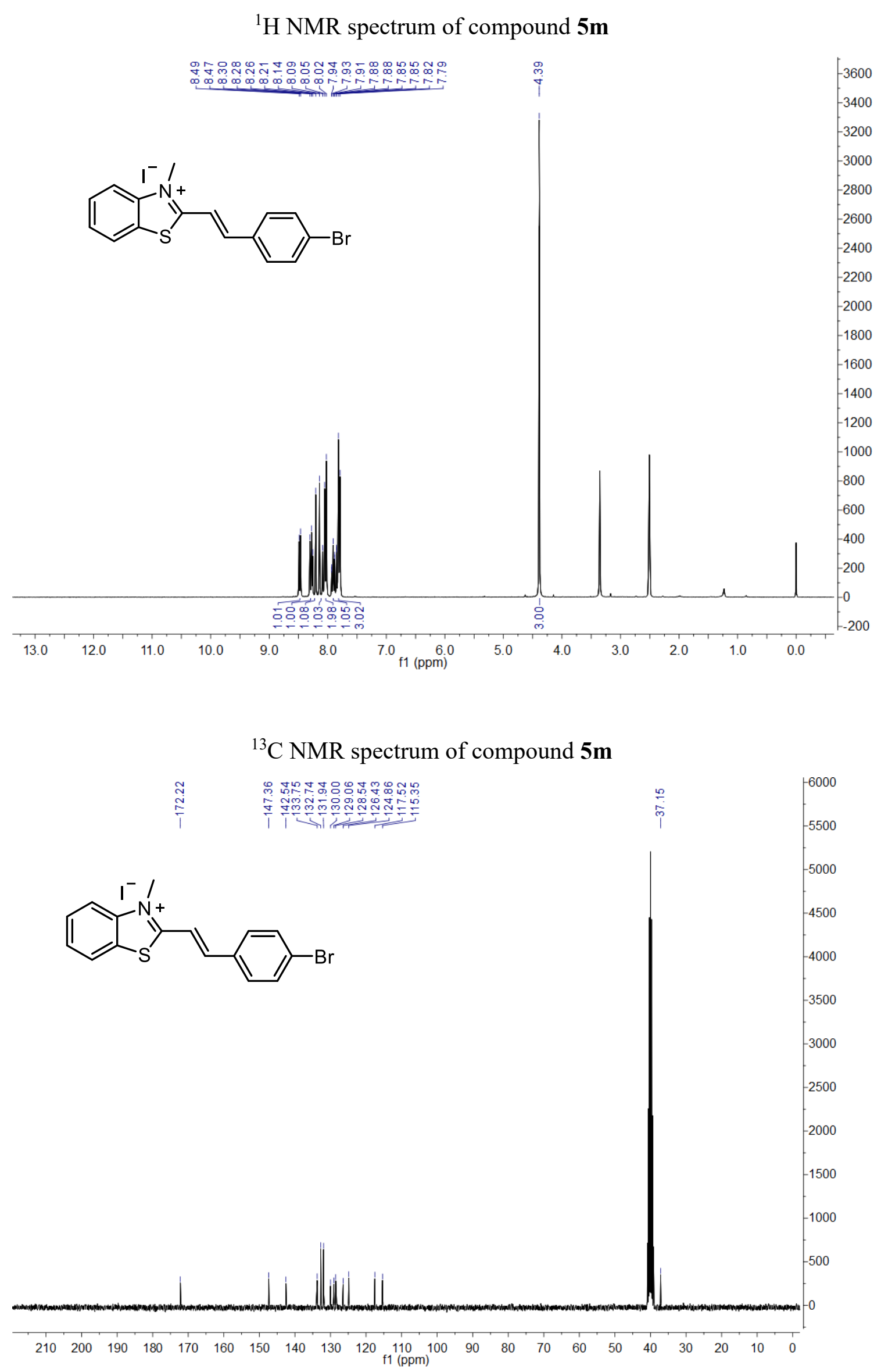


\section{HRMS of compound $\mathbf{5 m}$}

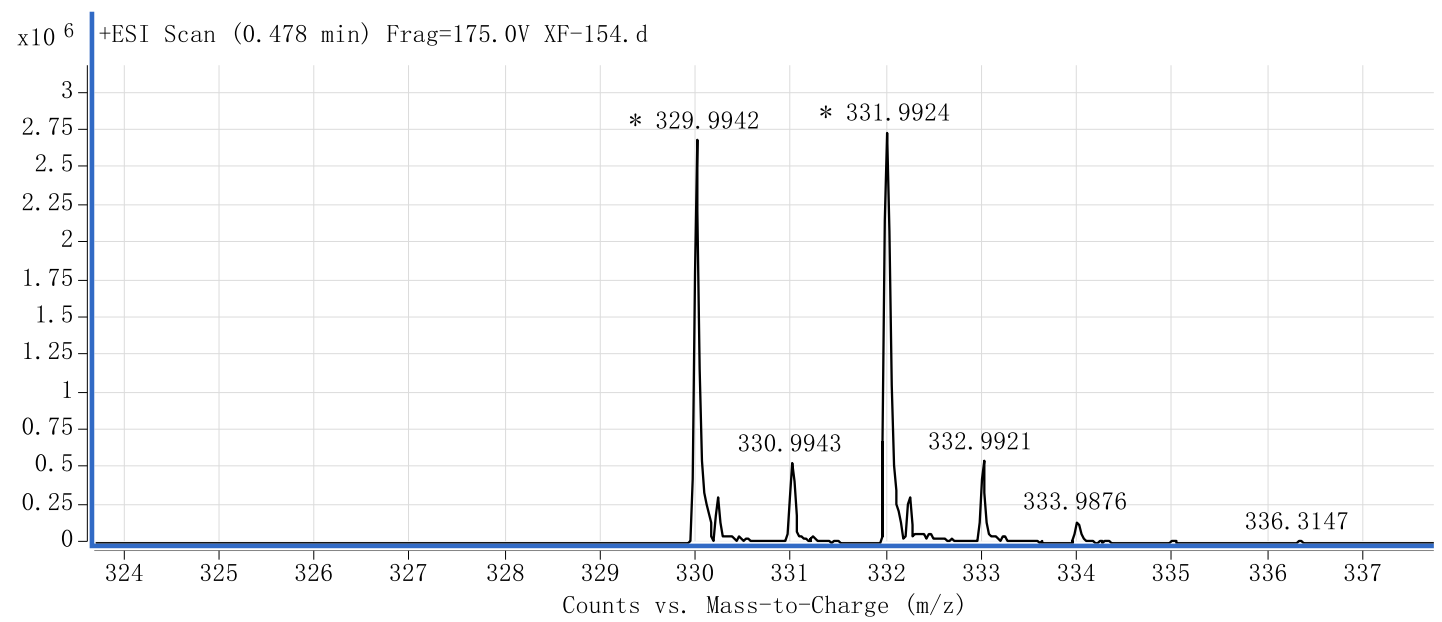

Hit Formula Calc m/z RDB ppm MS Rank MSMS ppm MSMS Rank Found

\begin{tabular}{|l|l|l|l|l|l|l|l|}
\hline 1 & $\mathrm{C}_{16} \mathrm{H}_{13} \mathrm{BrNS}^{+}$ & 329.9947 & 11.0 & -1.5 & 1 & & \\
\hline
\end{tabular}

\section{HPLC analysis of $\mathbf{5 m}$}

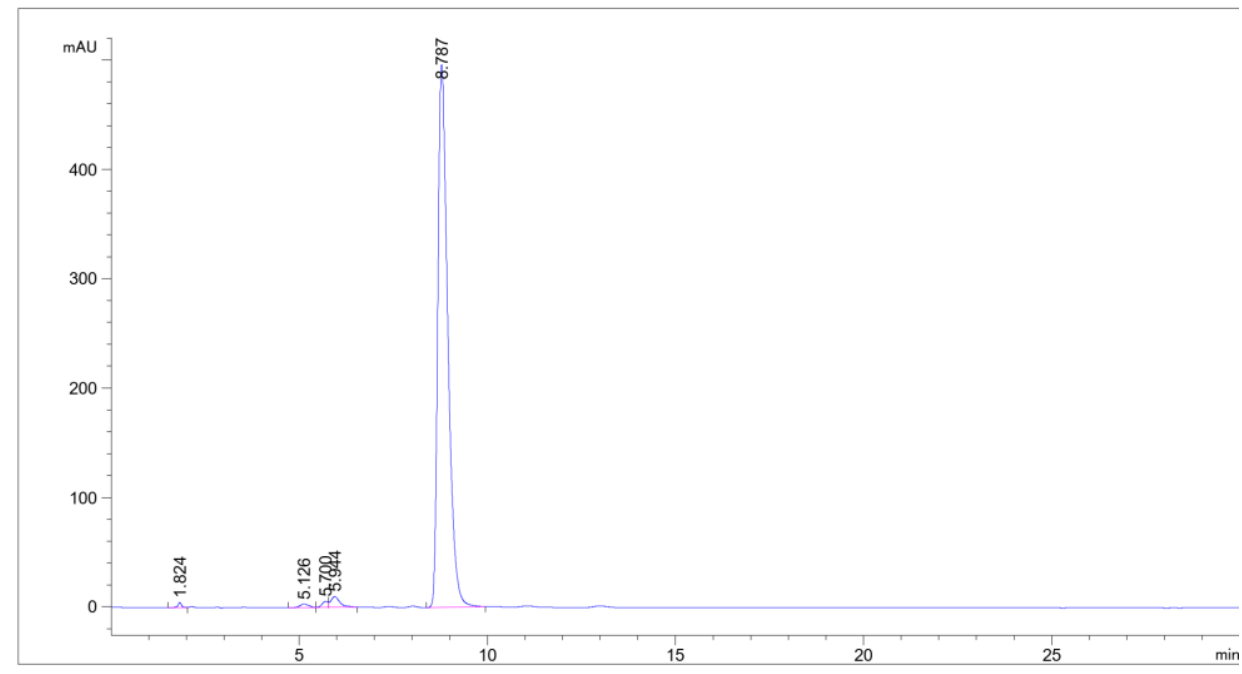

Area Percent Report

$\begin{array}{lcccc}\text { Sorted By } & : & \text { Signal } & \\ \text { Multiplier } & : & 2.0000 & \\ \text { Dilution } & : & 1.0000 & \\ \text { Sample Amount: } & & : \quad 10.00000 \quad \text { [ng/ul] } & \text { (not used in calc.) } \\ \text { Use Multiplier \& Dilution Factor with ISTDs } & \end{array}$

Signal 1: VWD1 A, Wavelength $=254 \mathrm{~nm}$

\begin{tabular}{|c|c|c|c|c|c|c|}
\hline $\begin{array}{c}\text { Peak } \\
\quad \#\end{array}$ & $\begin{array}{c}\text { RetTime } \\
\text { [min] }\end{array}$ & Type & $\begin{array}{l}\text { Width } \\
\text { [min] }\end{array}$ & $\begin{array}{c}\text { Area } \\
{\left[\mathrm{mAU}{ }^{\star} \mathrm{s}\right]}\end{array}$ & $\begin{array}{l}\text { Height } \\
\text { [mAU] }\end{array}$ & $\begin{array}{c}\text { Area } \\
\frac{\circ}{\partial}\end{array}$ \\
\hline 1 & 1.824 & BV & 0.1045 & 35.56487 & 4.85110 & 0.3758 \\
\hline 2 & 5.126 & BV & 0.2542 & 53.43095 & 3.22634 & 0.5646 \\
\hline 3 & 5.700 & vV & 0.1662 & 59.54366 & 5.46223 & 0.6291 \\
\hline 4 & 5.944 & VB & 0.2475 & 165.68379 & 9.83711 & 1.7506 \\
\hline 5 & 8.787 & BB & 0.2811 & 9150.06152 & 496.32071 & 96.6799 \\
\hline
\end{tabular}




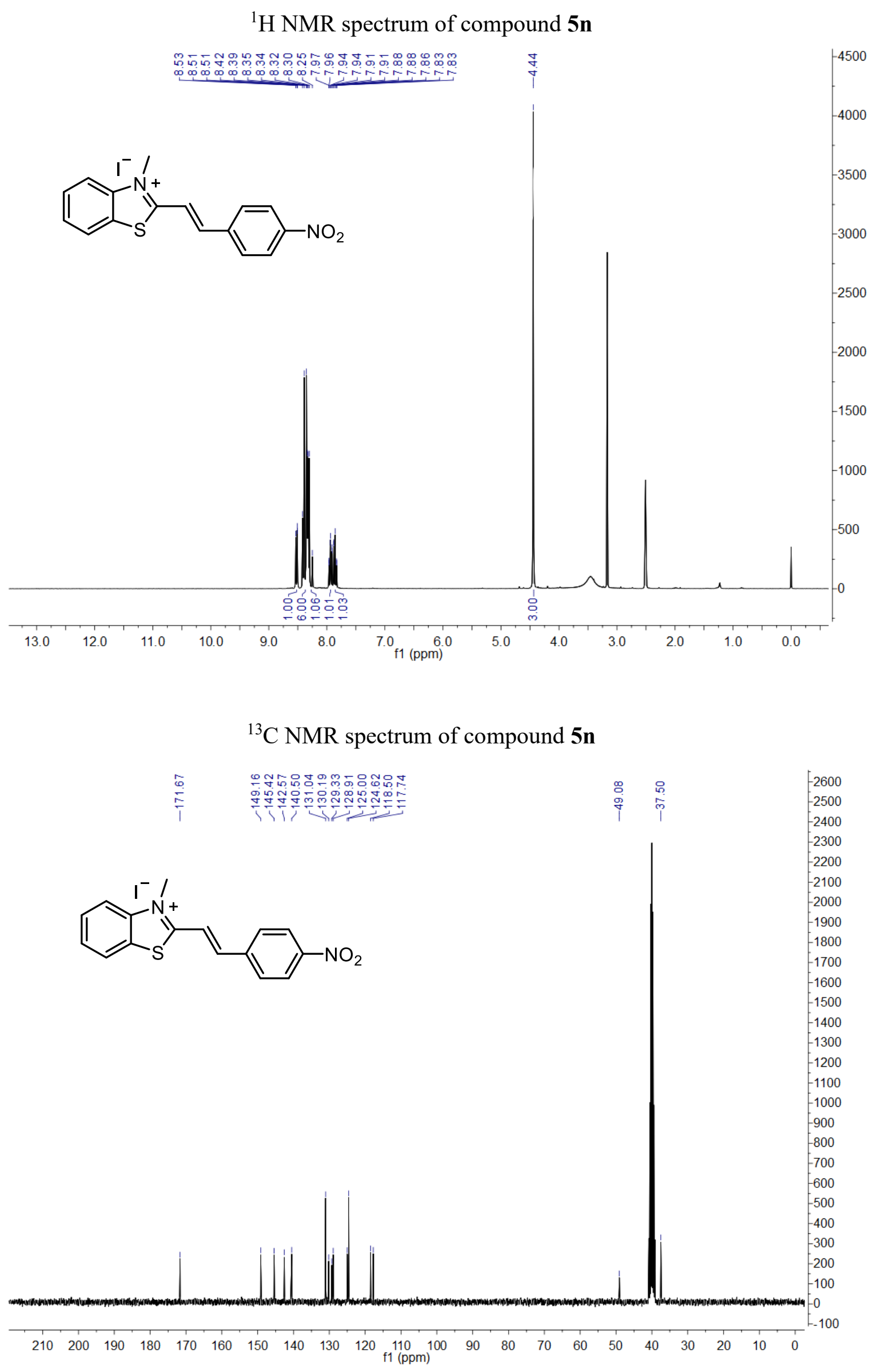




\section{HRMS of compound $\mathbf{5 n}$}

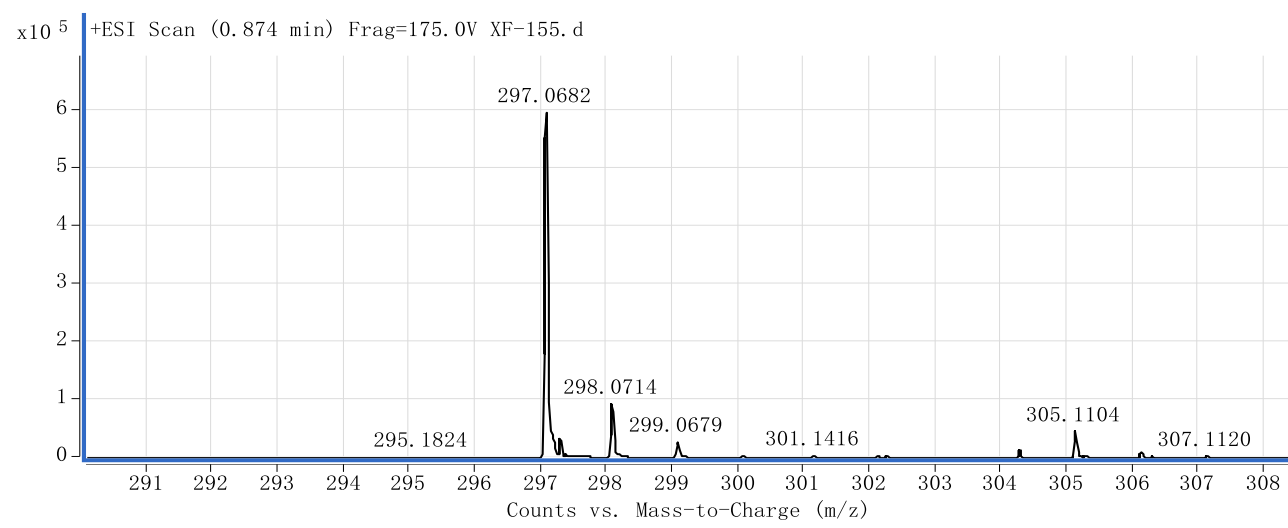

Hit Formula Calc m/z RDB ppm MS Rank MSMS ppm MSMS Rank Found

\begin{tabular}{|l|l|l|l|l|l|l|l|l|l|l|l|}
\hline 1 & $\mathrm{C}_{16} \mathrm{H}_{13} \mathrm{BrNS}^{+}$ & 297.0692 & 12.0 & -3.3 & 1 & & NA/NA
\end{tabular}

\section{HPLC analysis of $\mathbf{5 n}$}

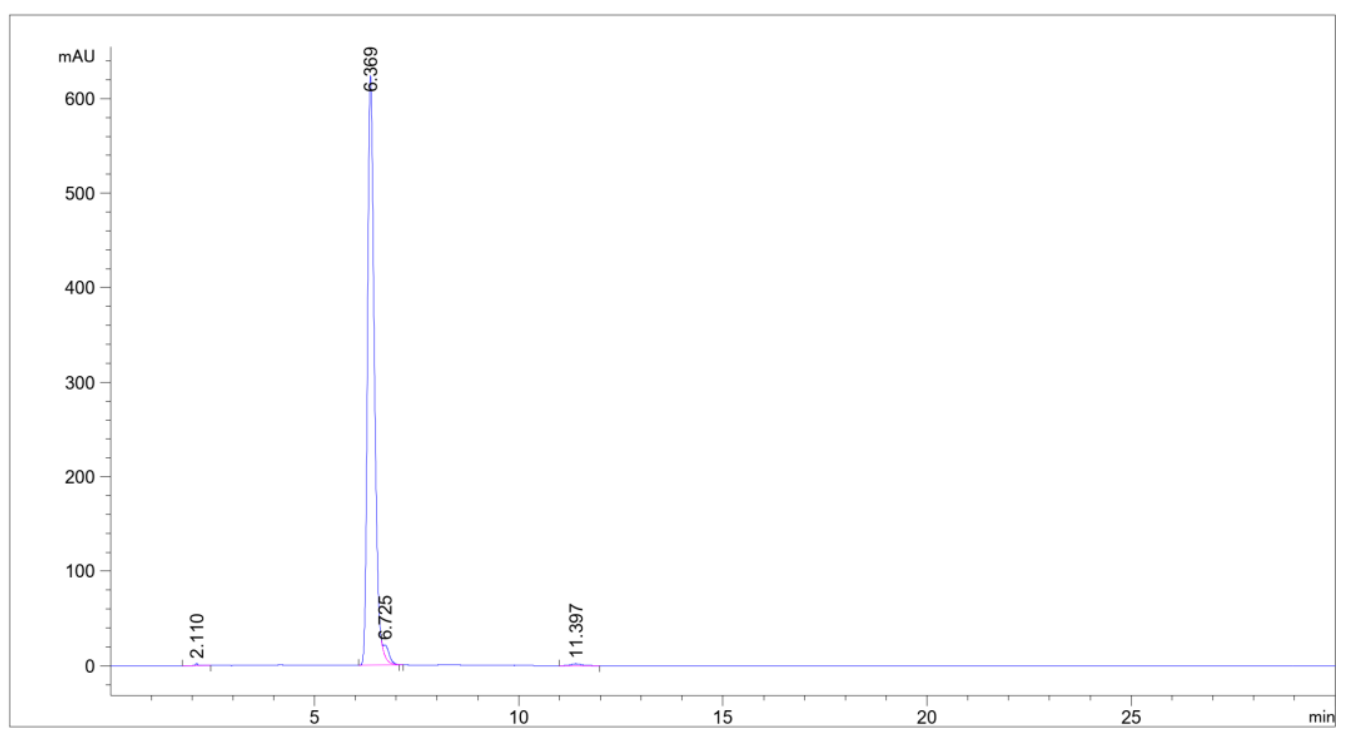

Area Percent Report

$\begin{array}{lll}\text { Sorted By } & : & \text { Signal } \\ \text { Multiplier } & : & 1.0000 \\ \text { Dilution } & : & 1.0000\end{array}$

Use Multiplier \& Dilution Factor with ISTDs

Signal 1: VWD1 A, Wavelength=254 nm

\begin{tabular}{|c|c|c|c|c|c|c|}
\hline $\begin{array}{c}\text { Peak } \\
\#\end{array}$ & $\begin{array}{c}\text { RetTime } \\
\text { [min] }\end{array}$ & Type & $\begin{array}{l}\text { Width } \\
\text { [min] }\end{array}$ & $\begin{array}{c}\text { Area } \\
{\left[\mathrm{mAU}{ }^{\star} \mathrm{s}\right]}\end{array}$ & $\begin{array}{l}\text { Height } \\
\text { [mAU] }\end{array}$ & $\begin{array}{c}\text { Area } \\
8\end{array}$ \\
\hline & & & & ---------- & ------- & ------1 \\
\hline 1 & 2.110 & BB & 0.0597 & 9.57940 & 2.34399 & 0.1275 \\
\hline 2 & 6.369 & BV $R$ & 0.1804 & 7345.61133 & 623.66968 & 97.7474 \\
\hline 3 & 6.725 & VB E & 0.1668 & 128.29704 & 11.10671 & 1.7072 \\
\hline 4 & 11.397 & $\mathrm{BB}$ & 0.2657 & 31.40487 & 1.82596 & 0.4179 \\
\hline otal & Is : & & & 7514.89264 & 638.94633 & \\
\hline
\end{tabular}



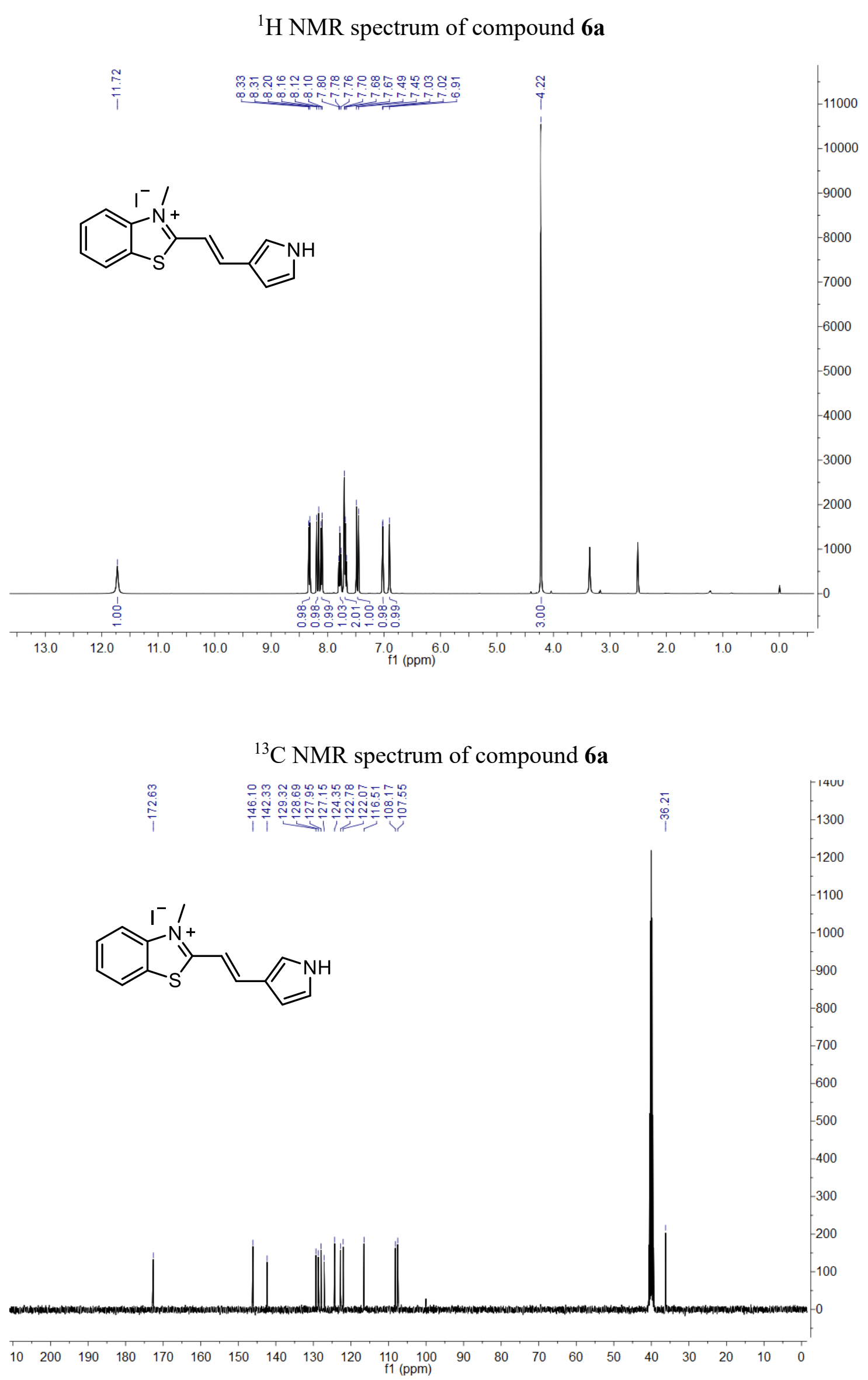
HRMS of compound $\mathbf{6 a}$

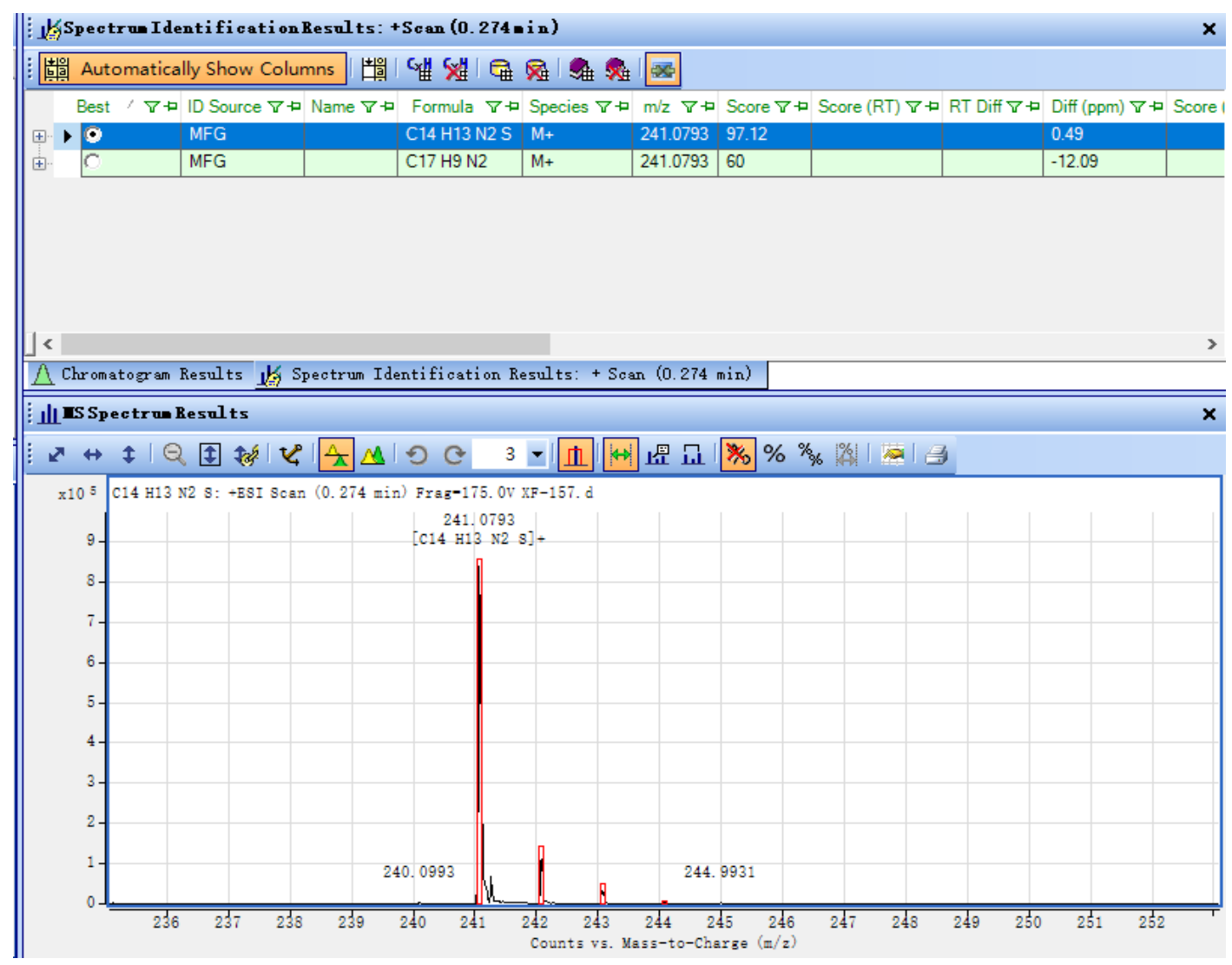




\section{HPLC analysis of $\mathbf{6 a}$}

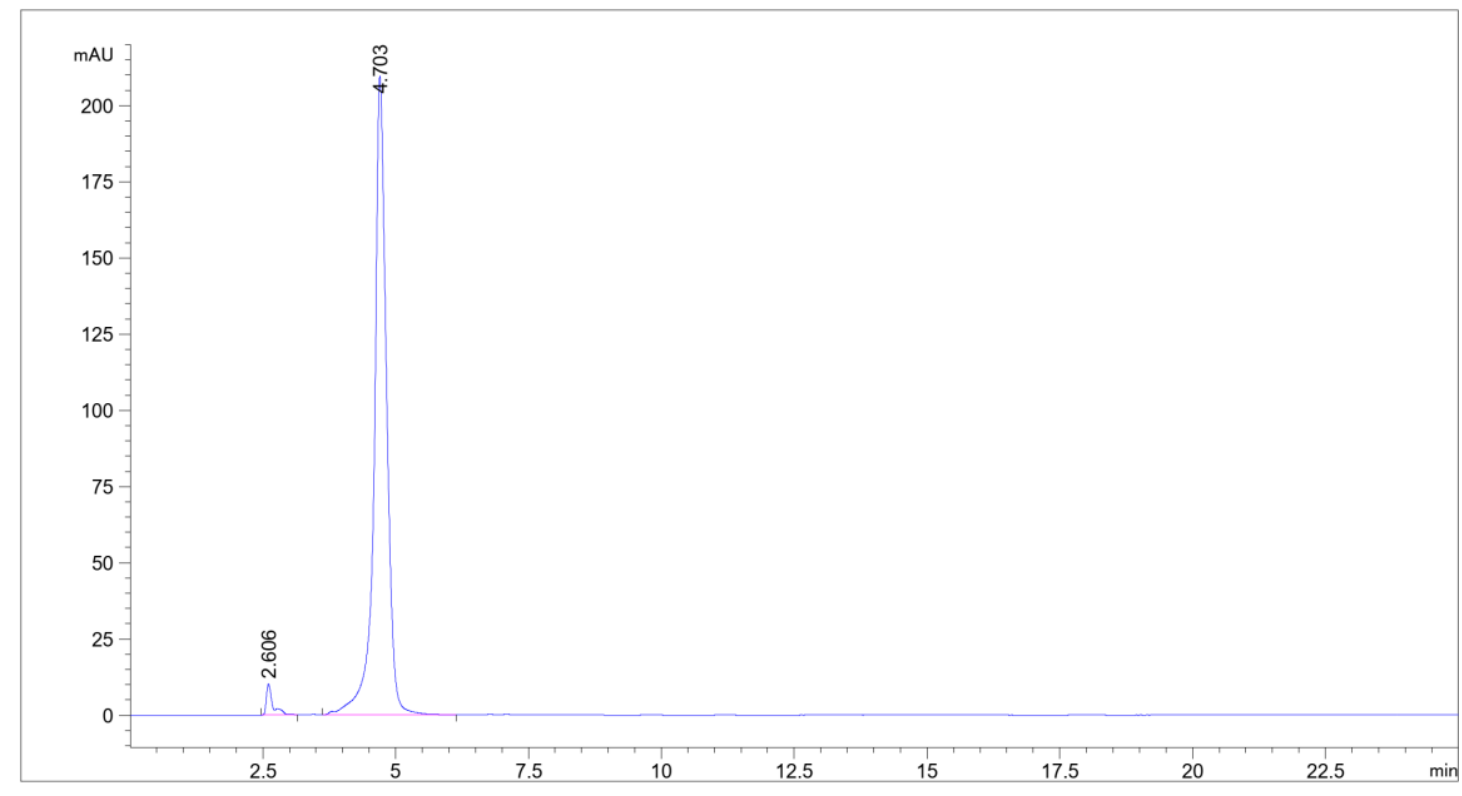

Area Percent Report

$\begin{array}{lll}\text { Sorted By } & : & \text { Signal } \\ \text { Multiplier } & : & 1.0000 \\ \text { Dilution } & : & 1.0000\end{array}$

Use Multiplier \& Dilution Factor with ISTDs

Signal 1: VWD1 A, Wavelength=254 nm

\begin{tabular}{|c|c|c|c|c|c|c|}
\hline $\begin{array}{c}\text { Peak } \\
\#\end{array}$ & $\begin{array}{c}\text { RetTime } \\
\text { [min] }\end{array}$ & Type & $\begin{array}{l}\text { Width } \\
\text { [min] }\end{array}$ & $\begin{array}{c}\text { Area } \\
{\left[\mathrm{mAU}^{*} \mathrm{~s}\right]}\end{array}$ & $\begin{array}{l}\text { Height } \\
\text { [mAU] }\end{array}$ & $\begin{array}{c}\text { Area } \\
\frac{\circ}{\gamma}\end{array}$ \\
\hline 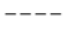 & & $|----|$ & ----- & --------- & --ー-ー-ー- & -------1 \\
\hline 1 & 2.606 & BV $R$ & 0.1231 & 83.27282 & 10.11140 & 2.4018 \\
\hline 2 & 4.703 & VB $R$ & 0.2354 & 3383.79150 & 209.49818 & 97.5982 \\
\hline Tota & $\cdot$ & & & 3467.06432 & 219.60959 & \\
\hline
\end{tabular}


${ }^{1} \mathrm{H}$ NMR spectrum of compound $\mathbf{6 b}$

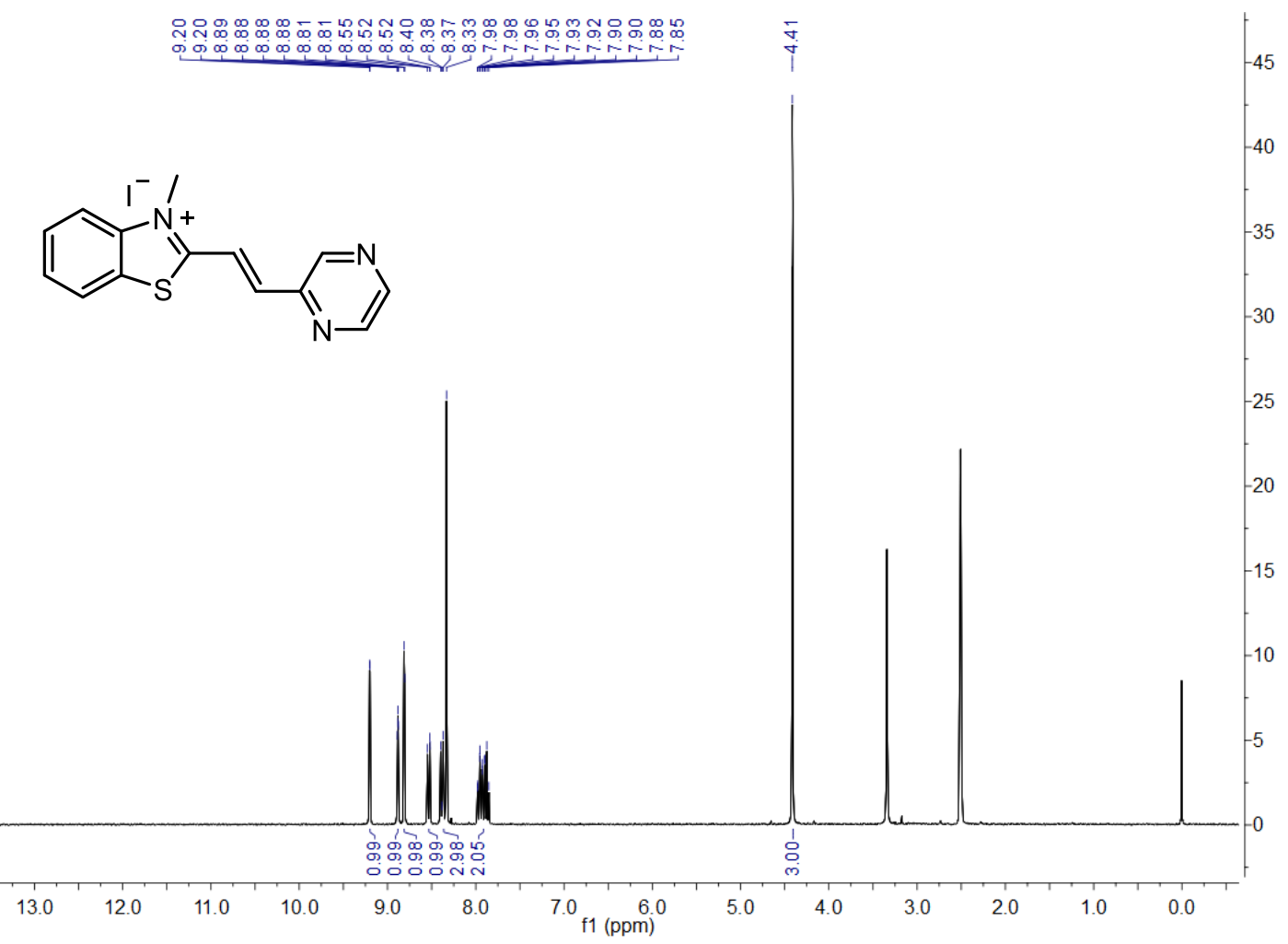

${ }^{13} \mathrm{C}$ NMR spectrum of compound $\mathbf{6 b}$

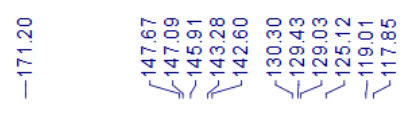
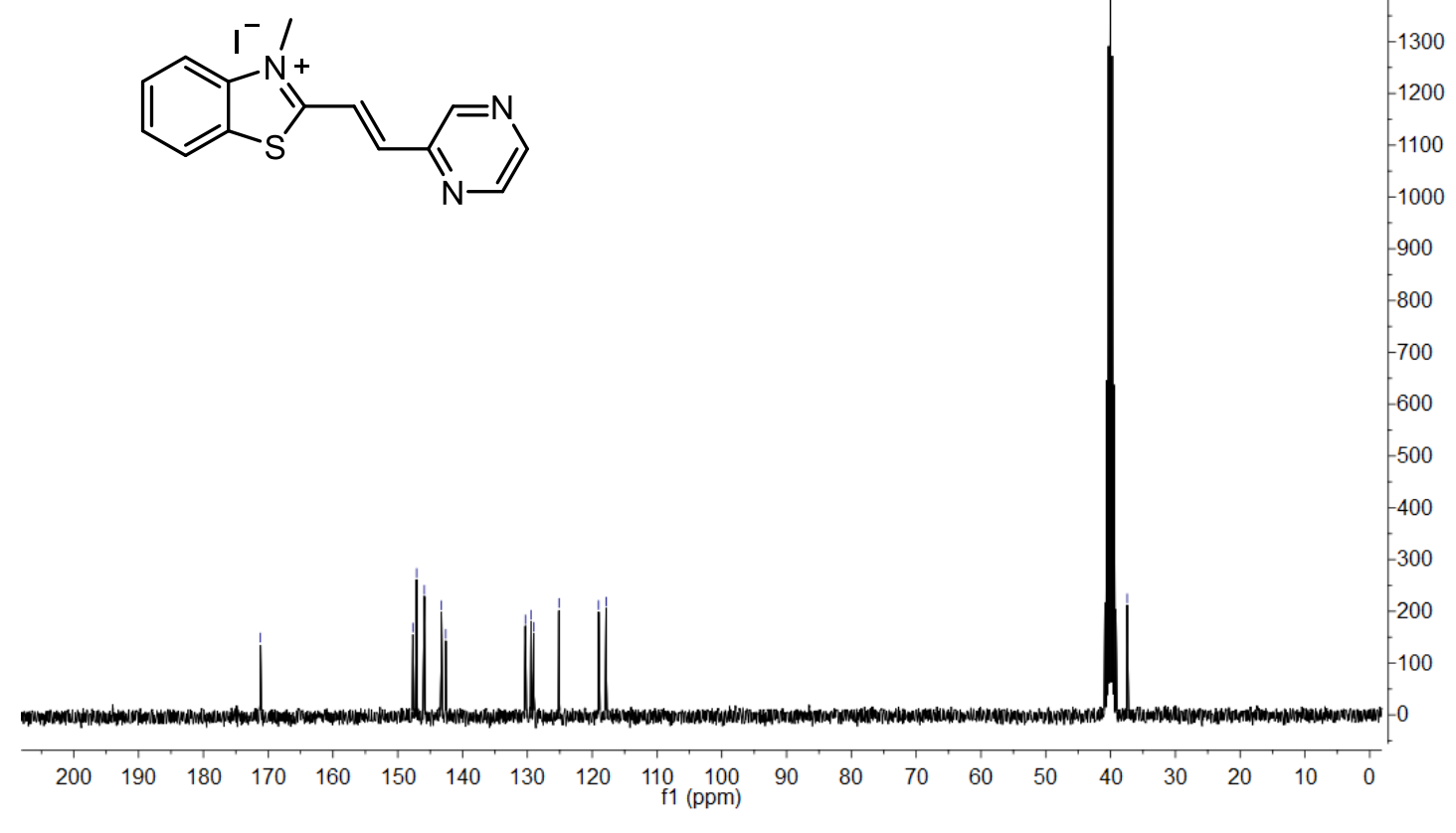
HRMS of compound $\mathbf{6 b}$

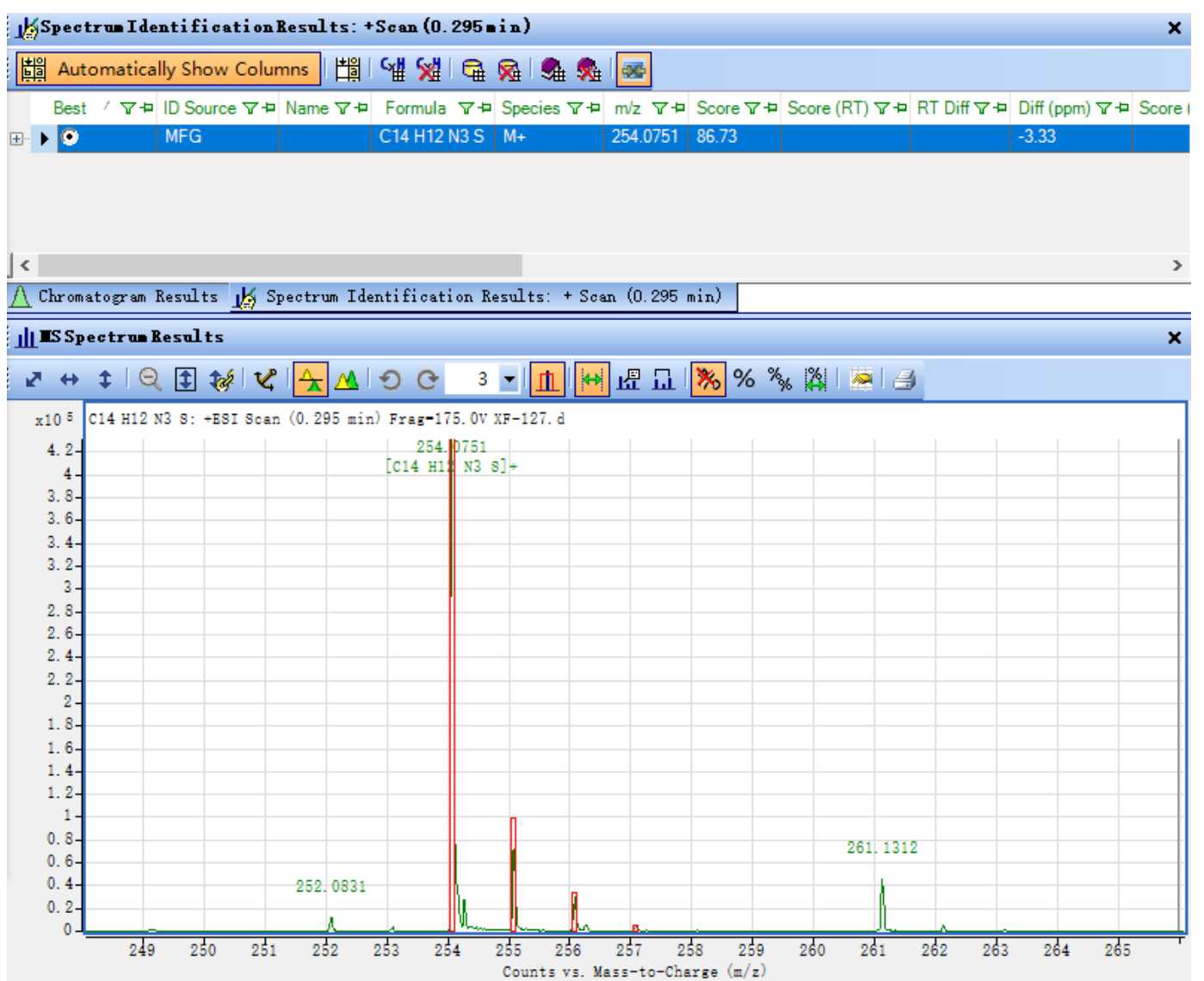




\section{HPLC analysis of $\mathbf{6 b}$}

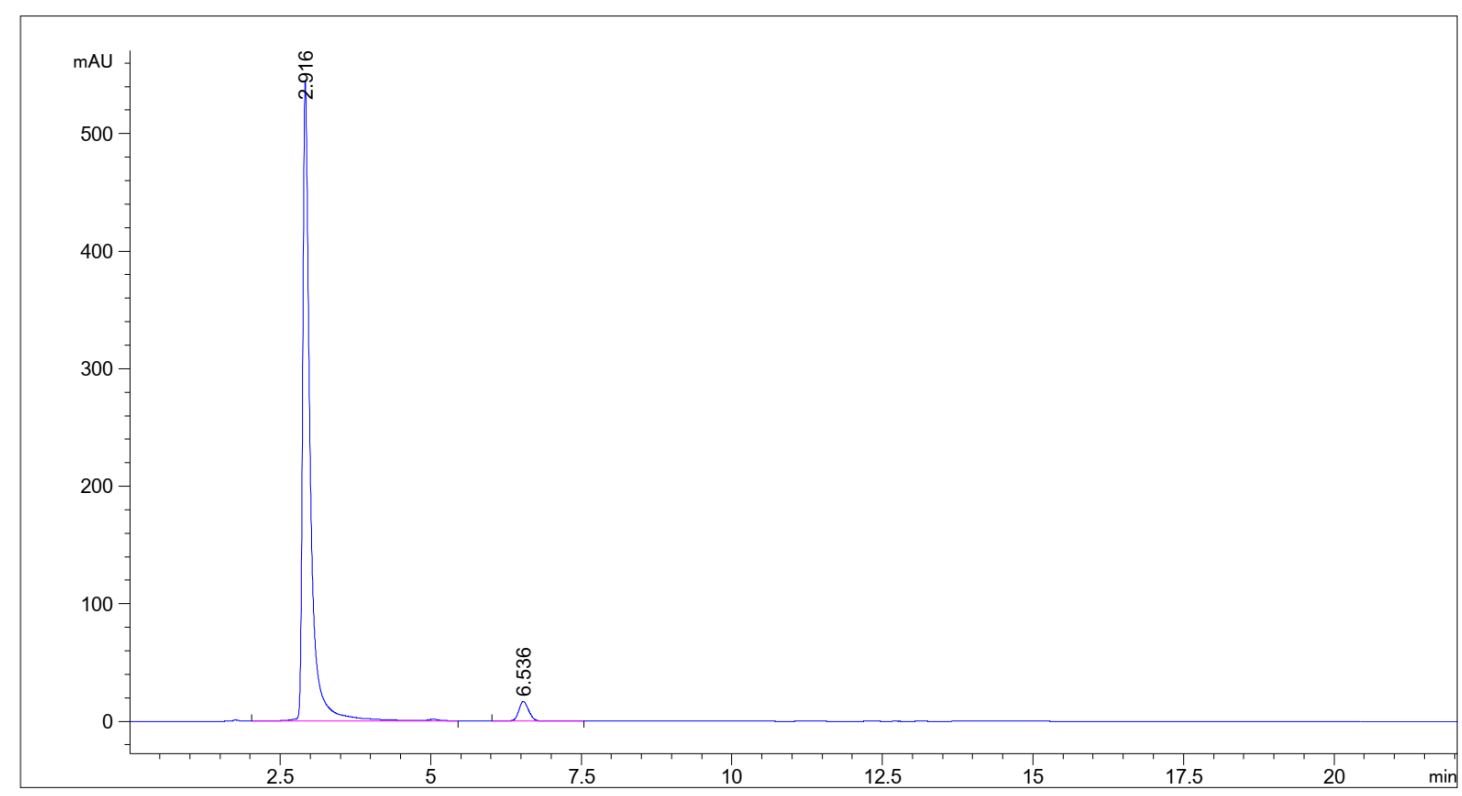

Area Percent Report

\begin{tabular}{|c|c|c|c|c|}
\hline Sorted By & : & Signal & & \\
\hline Multiplier & $:$ & 2.0000 & & \\
\hline Dilution & : & 1.0000 & & \\
\hline Sample Amount: & & $: \quad 10.00000$ & [ng/ul] & (not used in calc.) \\
\hline
\end{tabular}

Signal 1: VWD1 A, Wavelength=254 nm

\begin{tabular}{cccccc}
$\begin{array}{c}\text { Peak RetTime Type } \\
\text { Width } \\
\text { [min] }\end{array}$ & $\begin{array}{c}\text { Area } \\
{[\mathrm{min}]}\end{array}$ & $\begin{array}{c}\text { Height } \\
{\left[\mathrm{mAU}{ }^{*} \mathrm{~S}\right]}\end{array}$ & $\begin{array}{c}\text { Area } \\
{[\mathrm{mAU}]}\end{array}$ & \begin{tabular}{c}
$\circ$ \\
\hline
\end{tabular} \\
\hline 1 & 2.916 BV R & 0.1269 & 4750.19141 & 543.65112 & 96.0679 \\
2 & 6.536 VV R & 0.1769 & 194.42990 & 16.69632 & 3.9321
\end{tabular}

Totals :

$4944.62131 \quad 560.34745$ 

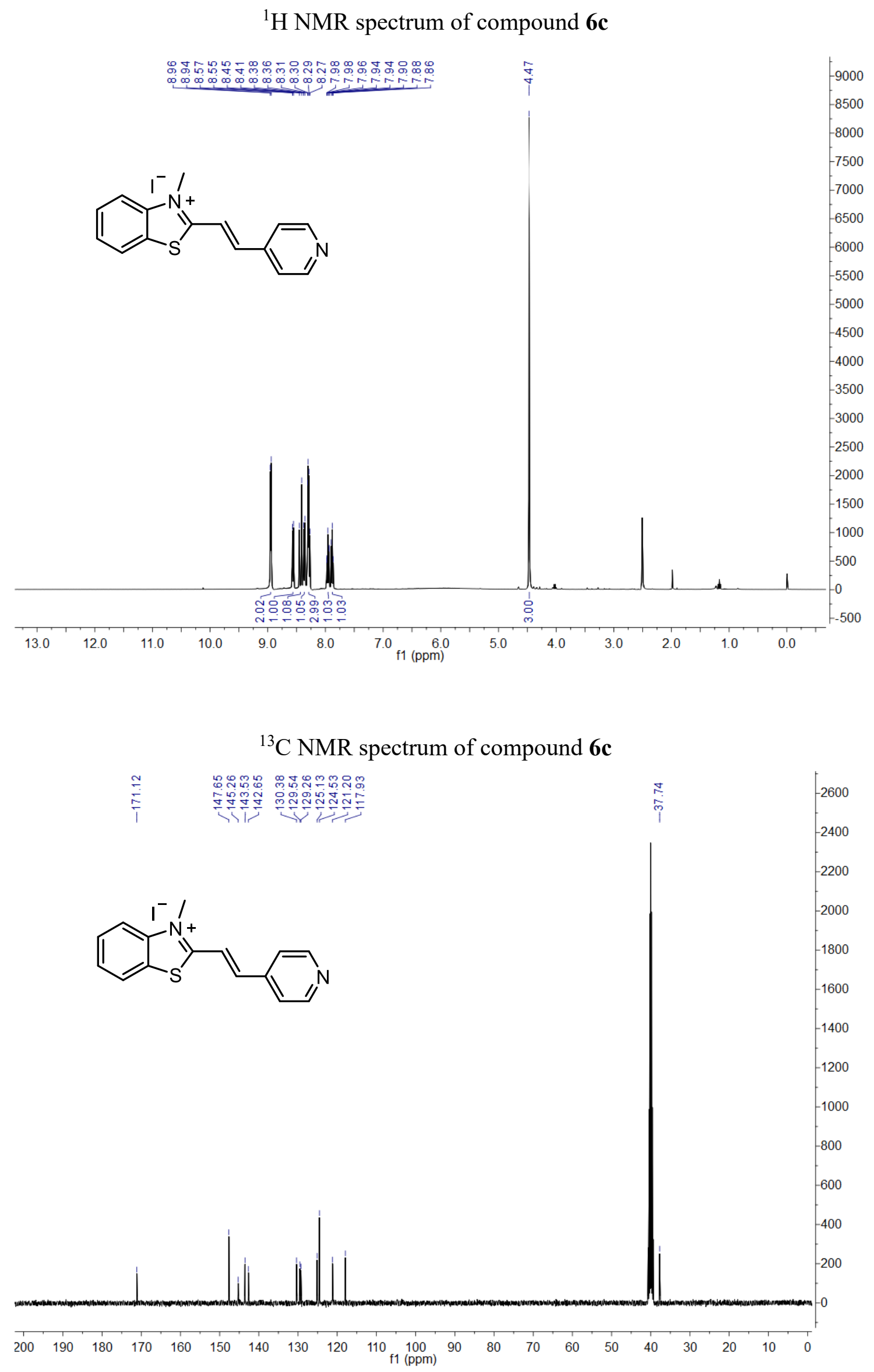
HRMS of compound $\mathbf{6 c}$

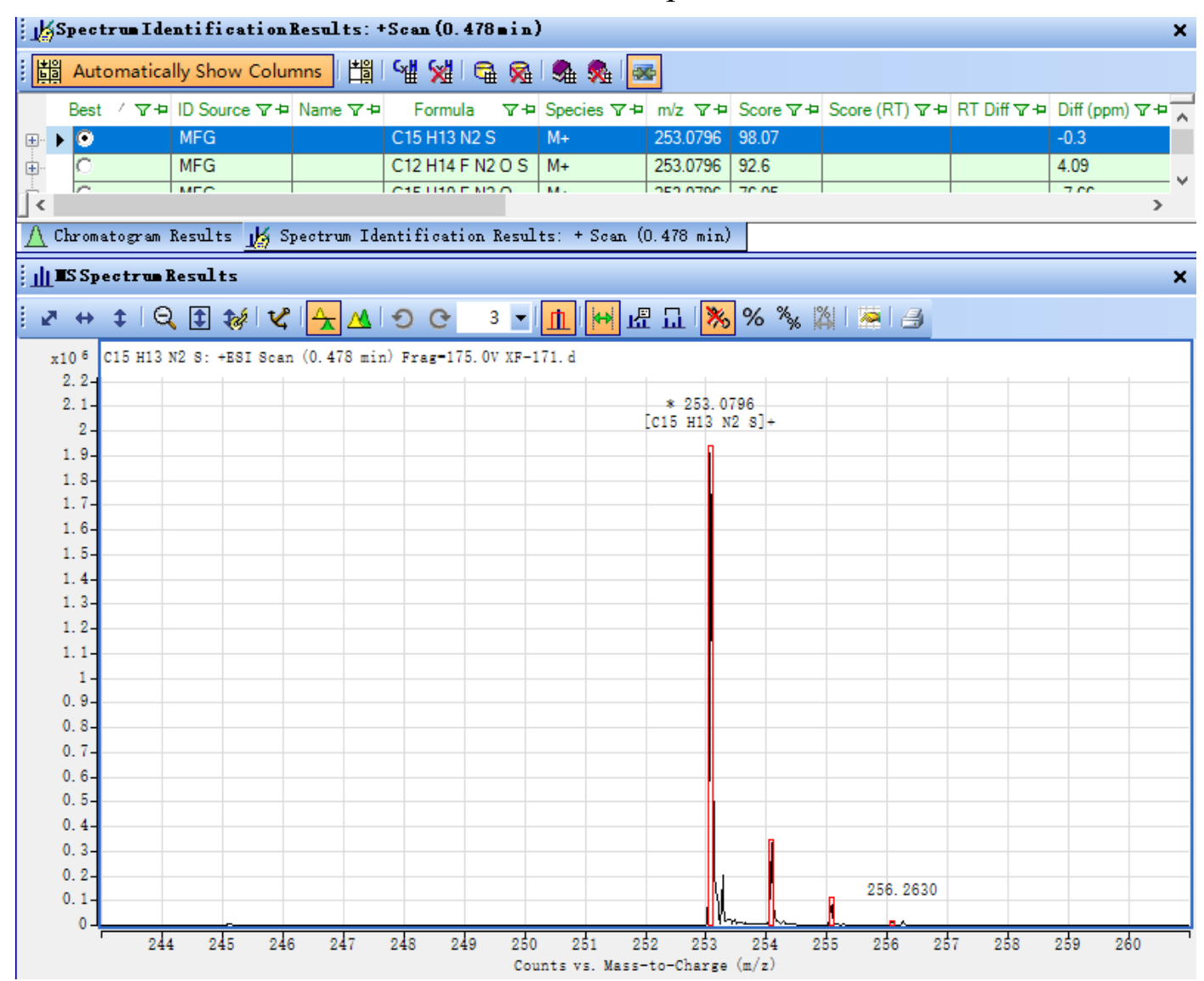


HPLC analysis of $\mathbf{6 c}$

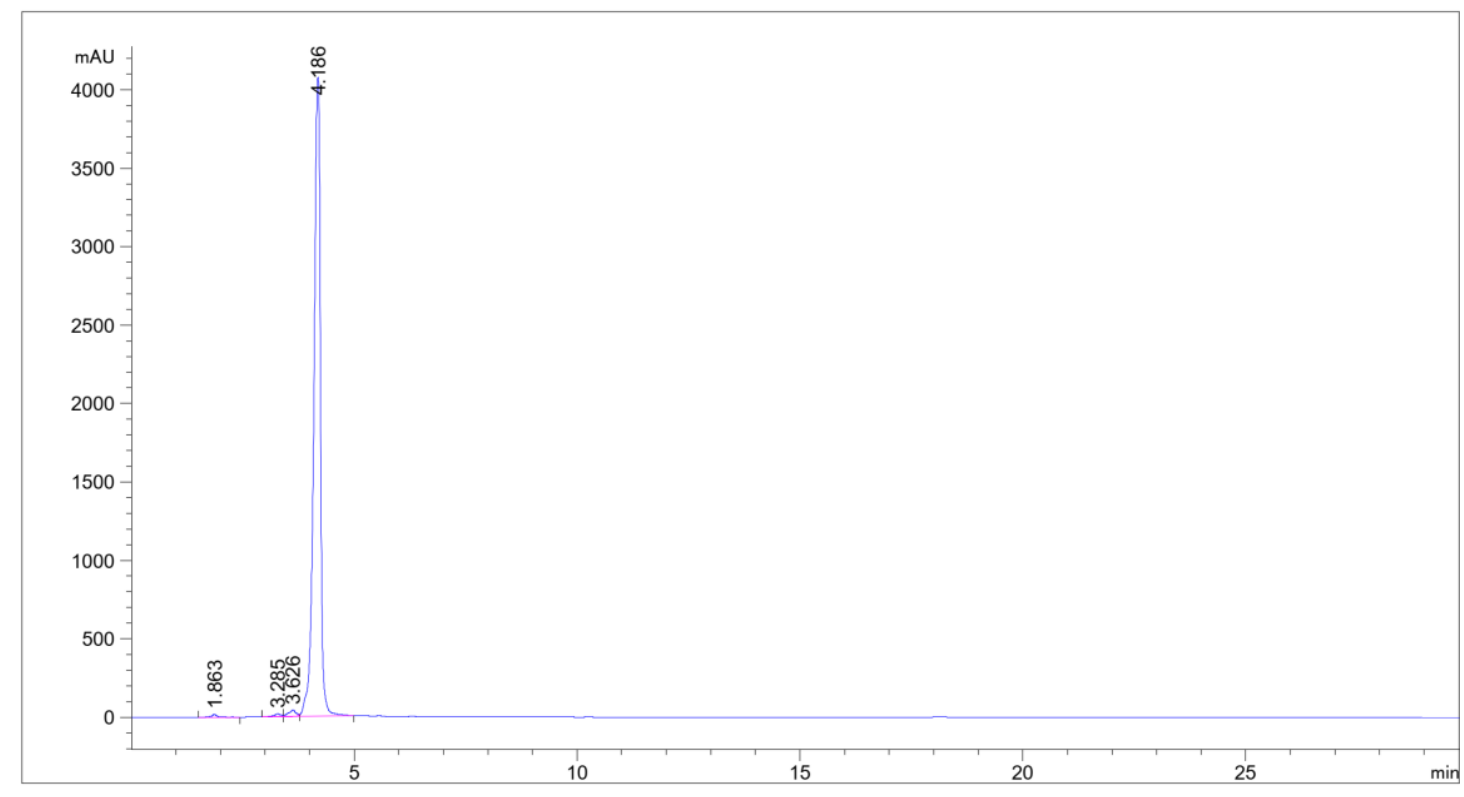

Area Percent Report

$\begin{array}{lcccc}\text { Sorted By } & : & \text { Signal } \\ \text { Multiplier } & : & 1.0000 \\ \text { Dilution } & : & 1.0000 & \\ \text { Sample Amount: } & & : & 5.00000 \quad \text { [ng/ul] } & \text { (not used in calc.) } \\ \text { Use Multiplier \& Dilution Factor with ISTDs }\end{array}$

Signal 1: VWD1 A, Wavelength=254 nm

\begin{tabular}{|c|c|c|c|c|c|c|}
\hline $\begin{array}{c}\text { Peak } \\
\#\end{array}$ & $\begin{array}{c}\text { RetTime } \\
\text { [min] }\end{array}$ & Type & $\begin{array}{l}\text { Width } \\
\text { [min] }\end{array}$ & $\begin{array}{c}\text { Area } \\
{\left[\mathrm{mAU}^{*} \mathrm{~s}\right]}\end{array}$ & $\begin{array}{l}\text { Height } \\
\text { [mAU] }\end{array}$ & $\begin{array}{c}\text { Area } \\
\frac{\circ}{0}\end{array}$ \\
\hline \multicolumn{7}{|c|}{ 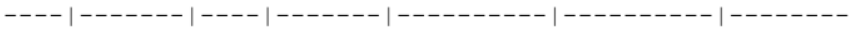 } \\
\hline 1 & 1.863 & VV R & 0.1286 & 155.48708 & 17.17041 & 0.3610 \\
\hline 2 & 3.285 & BV E & 0.1477 & 225.60411 & 20.44904 & 0.5238 \\
\hline 3 & 3.626 & VV E & 0.1543 & 467.91229 & 40.96156 & 1.0863 \\
\hline 4 & 4.186 & VB $R$ & 0.1570 & $4.22251 e 4$ & 4070.43042 & 98.0290 \\
\hline
\end{tabular}

